2002

\title{
Nucleation and coagulation of particulate matter inside a turbulent exhaust plume of a diesel vehicle
}

Donghee Kim

West Virginia University

Follow this and additional works at: https://researchrepository.wvu.edu/etd

\section{Recommended Citation}

Kim, Donghee, "Nucleation and coagulation of particulate matter inside a turbulent exhaust plume of a diesel vehicle" (2002). Graduate Theses, Dissertations, and Problem Reports. 2416.

https://researchrepository.wvu.edu/etd/2416 


\title{
NUCLEATION AND COAGULATION OF PARTICULATE MATTER INSIDE A TURBULENT EXHAUST PLUME OF A DIESEL VEHICLE
}

\author{
BY \\ DONGHEE KIM \\ Doctoral Dissertation submitted to the \\ College of Engineering and Mineral Resources at \\ West Virginia University \\ in partial fulfillment of the requirements for the degree of \\ Doctor of Philosophy \\ In \\ Mechanical Engineering
}

\author{
Approved by \\ Dr. Mridul Gautam, Chairman \\ Dr. Gary J. Morris \\ Dr. John M. Kuhlman \\ Dr. Charles Stanley \\ Dr. Alberto Ayala (CARB) \\ Dr. Aleksandar Bugarski (NIOSH) \\ Department of Mechanical Aerospace Engineering \\ Morgantown, West Virginia \\ 2002
}

Keywords: Nucleation, Coagulation, Condensation, Diesel particulate matter, Plume 


\section{ABSTRACT \\ NUCLEATION AND COAGULATION MODES IN THE FORMATION OF PARTICULATE MATTER INSIDE THE EXHAUST OF A DIESEL VEHICLE \\ Donghee Kim}

The objective of this study is to develop a physical model to accurately predict the nucleation, coagulation, and dynamics of particulate matter emission from diesel-fueled engines. The uniqueness of this research is that measured particulate matter (PM) size distribution data is not required a priori to solve the nucleation/coagulation equations; instead the PM concentration is predicted based on the fuel sulfur content, fuel to air ratio, exhaust flow rate, and the ambient conditions. This study presents the computational fluid dynamic modeling of an exhaust plume dispersed from a stack pipe of a tractor truck powered by a $330 \mathrm{HP}(246 \mathrm{~kW})$ diesel engine. This effort uses the $\mathrm{k}-\varepsilon$ eddy dissipation model to accurately predict the variation of carbon dioxide concentration coming out of the stack pipe into the ambient. A specific goal of this effort is to study the effect of the recirculation region near the truck walls on dispersion of $\mathrm{CO}_{2}$ concentration. The predicted results showed an excellent agreement with the experimentally measured values of $\mathrm{CO}_{2}$ concentration variation, dilution ratio, and the temperature variations in the wind tunnel. It was predicted that the relative concentration of $\mathrm{CO}_{2}$ coming out of the stack dropped rapidly from 1 to 0.01 within a distance of $100^{\prime \prime}(2.54 \mathrm{~m})$ downstream of the exhaust outlet.

Additionally, the simultaneous effects of nucleation, condensation and coagulation are incorporated in predicting the PM emissions from on-road heavy-duty diesel vehicles. The nucleation rates in the formation of PM are calculated directly from the sulfur content in the fuel. It was predicted that the critical nucleus diameter decreased by approximately $30 \%$ and the number concentration increased by a factor of 6 with the increase in relative humidity from $10 \%$ to $90 \%$ for a fuel with $50 \mathrm{ppm}$ sulfur content. The numerical simulations clearly suggested that the condensation effects are very important near the stack outlet where the rapid dilution of particulate matter with the ambient is dominant. Ignoring the contribution from condensation term decreased PM count median diameter from approximately $52 \mathrm{~nm}$ to $10 \mathrm{~nm}$. The root mean square (RMS) error in the numerically predicted particle number concentration was within 14.3 $\%$ of the experimentally measured values. An increase in CMD from $52 \mathrm{~nm}$ to $62 \mathrm{~nm}$ was predicted for a distance of $0.51 \mathrm{~m}(20$ ") from the stack exit to $8.56 \mathrm{~m}$ (337") from the stack exit, and the number concentration for the same distance decreased from $8.77 \mathrm{E}+6$ to $2.1 \mathrm{E}+5$ No. $/ \mathrm{cm}^{3}$. 


\section{ACKNOWLEDGMENTS}

I would like to thank Professor Mridul Gautam for giving me an opportunity to work on an exciting project, and for providing me with support, and advice during this time. I would like to thank Professor Gary Morris, Professor John Kuhlman, Professor Charles Stanley, Dr. Alberto Ayala, and Dr. Aleksandar Bugarski for serving on my committee and giving me an excellent advice and suggestions in improving this work.

I would like to thank Mr. Sandeep Mehta for giving me all the MOUDI and SMPS data from the Langley tunnel study. I would like to thank Mr. Dan Carder and WVU Engine and Emissions Research Center and WVU Transportable Heavy-duty vehicle Emission Testing Laboratory's staff for their help.

Last, but not least, I would like to sincerely thank my family for their love, absolute support and sacrifice, which helped me complete this work. 


\section{Table of Contents}

Chapter

Page

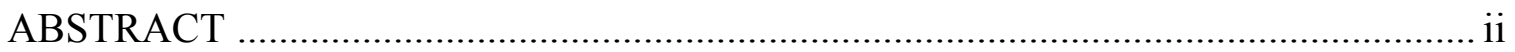

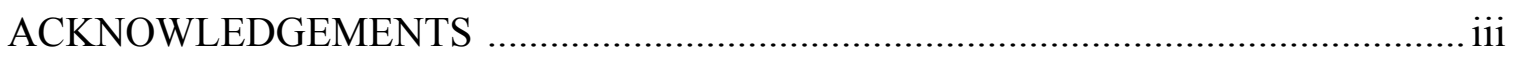

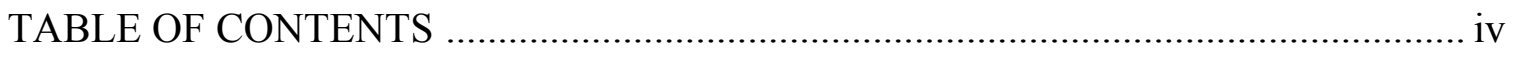

LIST OF FIGURES ........................................................................................ vii

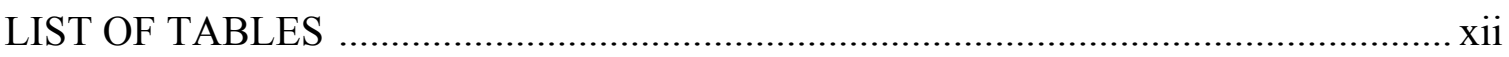

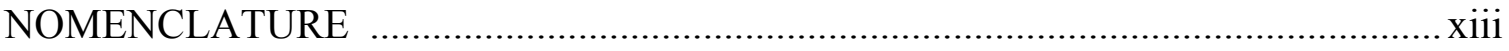

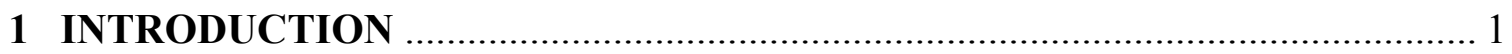

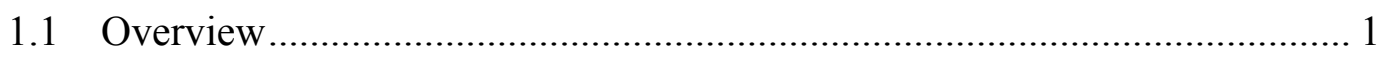

1.2 Objectives of this Research ................................................................. 3

1.3 Technical Approach to Accomplish Objectives ...................................... 6

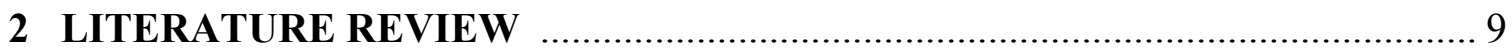

2.1 HEALTH HAZARDS OF DIESEL EXHAUST .................................... 9

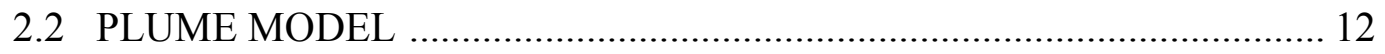

2.2.1 Empirical Gaussian Models ................................................... 14

2.2.2 Similarity Models ............................................................. 16

2.2.3 Probability Density Function Models ........................................ 17

2.2.4 Statistical Models................................................................... 18

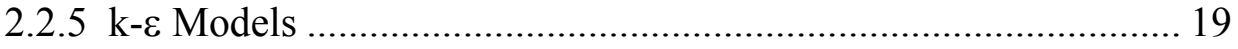

2.2.6 Large Eddy Simulation Models .............................................. 19

2.3 CHARACTERISTICS OF DIESEL PARTICULATES MATTER ............. 21

2.4 OVERVIEW OF SMPS AND PM SAMPLING TECHNIQUES ................ 28

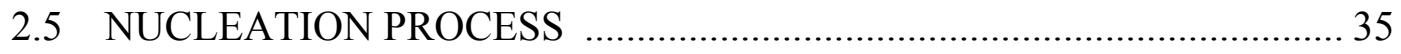

2.5.1 Homogeneous Nucleation ..................................................... 35 
2.5.2 Heterogeneous Nucleation ........................................................ 36

2.5.3 Binary Homogeneous Nucleation in the $\mathrm{H}_{2} \mathrm{SO}_{4}-\mathrm{H}_{2} \mathrm{O}$

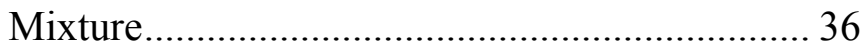

2.5.3.1 Effect of Saturation Ratio and

Humidity on Nucleation...................................... 40

2.5.3.2 Effect of Dilution Ratio on Diesel

Particulate Matter................................................ 41

2.5.3.3 Kelvin Effects on Nucleation .............................. 44

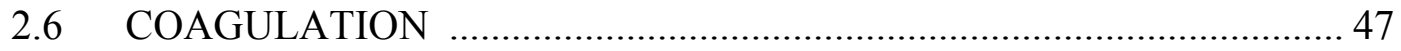

2.6.1 Techniques for solving coagulation governing equations .............51

2.6.1.1 "J-space" transformation............................................. 51

2.6.1.2 Asymptotic Solution ................................................. 53

2.6.1.3 Discrete Method...................................................... 54

2.6.1.4 Moment Method ...................................................... 55

2.6.1.5 Parametrized Representation .................................... 56

2.6.1.6 Similarity Solution .................................................. 58

2.6.1.7 Direct Simulation

by Monte Carlo Method............................................... 60

2.6.2 Other Coagulation Models in the Literature ................................ 60

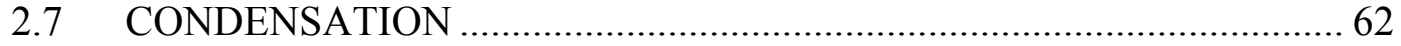

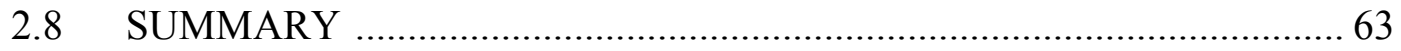

3 THEORETICAL FORMULATION \& GOVERNING EQUATIONS ................. 67

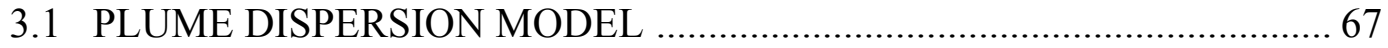

3.1.1 Gaussian Model ...................................................................... 70

3.1.2 Probability Density Function (PDF) Model................................. 73

3.2 DISCRETE PARTICLE DYNAMICS ………………........................... 75

3.2.1 Nucleation Model.................................................................... 76 


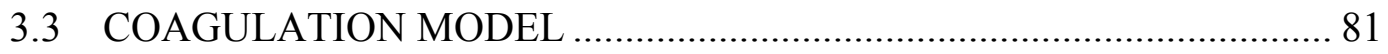

3.3.1 Coagulation Kernel ............................................................... 87

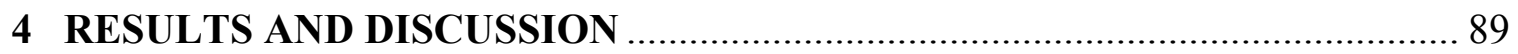

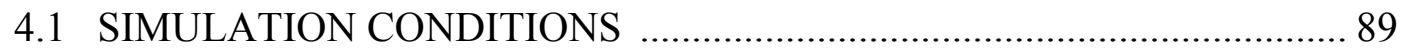

4.2 PLUME PREDICTIONS .......................................................................... 90

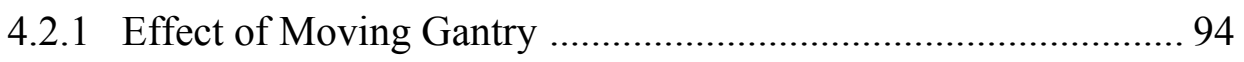

4.2.2 Effect of Tractor-Trailer ........................................................ 95

4.2.3 Simulation Results using Gaussian and PDF Models .............. 109

4.3 EFFECT OF NUCLEATION ON PM FORMATION ............................ 113

4.4 EFFECT OF COAGULATION ON PM SIZE DISTRIBUTION ............. 141

4.5 EFFECTS OF NUCLEATION, CONDENSATION \& COAGULATION ON PM SIZE DISTRIBUTIONS AND CONCENTRATION .................. 147

5 CONCLUSIONS AND FUTURE RECOMMENDATIONS ......................... 160

5.1 FUTURE RECOMMENDATIONS ..................................................... 163

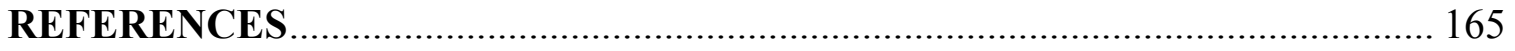

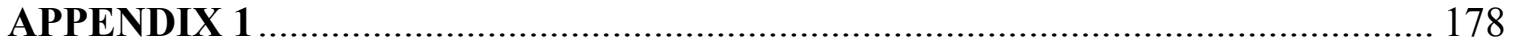

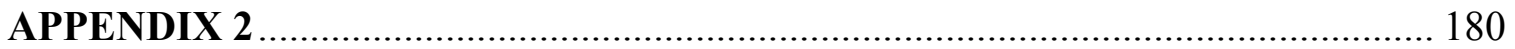

PUBLICATIONS RESULTING FROM THIS WORK...................................... 198 


\section{LIST OF FIGURES}

Figure 2.1

Figure 2.2

Figure 2.3

Figure 2.4

Figure 2.5

Figure 2.6

Figure 2.7

Figure 2.8

Figure 4.1

Figure 4.2

Figure 4.3

Figure 4.4
Engine exhaust mass and number weighted size distributions

shown with alveolar deposition (Kittelson, 1998).... 10

Typical engine exhaust particle size distribution for different

buses at different locations (Gautam and Mehta, 2001)

Schematic of the particle and gas phase processes and factors affecting the particle size distributions and chemical composition of the particles from the time of combustion to discharge into the atmosphere, followed by atmospheric transport and transformation

(Baumgard and Johnson, 1996) 24

Typical structure of engine exhaust particles (Kittelson, 1998) .. 27

Schematic test setup in Langley wind tunnel 30

Events leading to binary homogeneous nucleation and particle growth (Baumgard and Johnson, 1996) 39

Gibbs free energy charge for formation of a droplet radius $\mathrm{Rp}$ from a vapor with a saturation ratio S (Seinfeld and Pandis, 1997) 45

The ratio of the vapor pressure $\mathrm{p}_{\mathrm{A}}$ of a solvent $\mathrm{A}$ over a solution droplet to its pure component vapor pressure over a flat surface $\mathrm{p}_{\mathrm{A}}{ }^{0}$, as a function of the diameter of the droplet. Curves of this type are called Köhler curves (Seinfeld and Pandis, 1997) .......... 48 Schematic grid of the truck inside wind tunnel 96 Computational grid of the truck inside wind tunnel

Filled contours of relative concentration of $\mathrm{CO}_{2}$

inside the tunnel 98

Contours of relative concentration of $\mathrm{CO}_{2}$ inside

the tunnel on a plane passing through

the exhaust pipe 99 
Figure 4.5

Figure 4.6

Figure 4.7

Figure 4.8

Figure 4.9

Figure 4.10

Figure 4.11

Figure 4.12

Figure 4.13

Figure 4.14

Figure 4.15

Figure 4.16

Figure 4.17

Figure 4.18

Figure 4.19

Figure 4.20
Variation of relative concentration of $\mathrm{CO}_{2}$

along the centerline of plume

Velocity vectors showing recirculation near

the exhaust pipe of the truck

Variation of $\mathrm{CO}_{2}$ concentration inside the plume

perpendicular to the centerline

Variation of dilution ratio of $\mathrm{CO}_{2}$ along the

centerline of plume

Variation of temperature along the centerline of plume

Relative concentration of $\mathrm{CO}_{2}$ near the moving gantry

inside wind tunnel

Variation of $\mathrm{CO}_{2}$ concentration inside the plume perpendicular

to the centerline near the moving gantry

Computational grid of tractor-trailer

Contours of relative concentration (dimensionless) of $\mathrm{CO}_{2}$

on the tractor-trailer

Variation of $\mathrm{CO}_{2}$ concentration inside the plume perpendicular

to the centerline

Variation of $\mathrm{CO}_{2}$ concentration inside the plume perpendicular

to the centerline

Effect of relative humidity on nucleation rate and

nucleus size

Nucleation rate as a function of dilution tunnel $\mathrm{H}_{2} \mathrm{SO}_{4}$

vapor pressure at a constant $20 \%$ relative humidity and

temperature 25 and $46{ }^{\circ} \mathrm{C}$

Effect of critical nucleation diameter as a function of

relative humidity

Effect of nucleation rate as a function of relative

humidity

Effect of relative humidity on sulfuric acid vapor pressure

with time for $13.1 \mathrm{ppm}$ sulfur fuel 
Figure 4.21

Figure 4.22

Figure 4.23

Figure 4.24

Figure 4.25

Figure 4.26

Figure 4.27

Figure 4.28

Figure 4.29

Figure 4.30

Figure 4.31

Figure 4.32

Figure 4.33

Figure 4.34

Figure 4.35
Effect of relative humidity on sulfuric acid vapor pressure with time for $125 \mathrm{ppm}$ sulfur fuel

Effect of relative humidity on sulfuric acid vapor pressure

with time for $300 \mathrm{ppm}$ sulfur fuel

Effect of relative humidity on nucleus diameter

for $13.1 \mathrm{ppm}$ sulfur fuel

Effect of relative humidity on nucleus diameter

for $125 \mathrm{ppm}$ sulfur fuel

Effect of relative humidity on nucleus diameter

for 300 ppm sulfur fuel

Effect of relative humidity on nucleation rate with time

for $13.1 \mathrm{ppm}$ fuel sulfur

Effect of relative humidity on nucleation rate with time

for $125 \mathrm{ppm}$ Sulfur fuel.

Effect of relative humidity on nucleation rate with time

for 300 ppm sulfur fuel

Effect of sulfur fuel content on nucleation rate with time

for $20 \%$ relative humidity

Effect of sulfur fuel content on nucleation rate with time

for $35 \%$ relative humidity

Effect of fuel sulfur content on nucleation rate with time

for $45 \%$ relative humidity

Effect of fuel sulfur content on nucleus diameter with time

for $20 \%$ relative humidity

Effect of fuel sulfur content on nucleus diameter with time

for $35 \%$ relative humidity

Effect of fuel sulfur content on nucleus diameter with time

for $45 \%$ relative humidity

Effect of fuel sulfur content on sulfuric acid vapor pressure with time for $20 \%$ relative humidity 138 
Figure 4.36

Figure 4.37

Figure 4.38

Figure 4.39

Figure 4.40

Figure 4.41

Figure 4.42

Figure 4.43

Figure 4.44

Figure 4.45

Figure 4.46
Effect of fuel sulfur content on sulfuric acid vapor pressure with time for $35 \%$ relative humidity.

Effect of fuel sulfur content on sulfuric acid vapor pressure

with time for $45 \%$ relative humidity $45 \%$.

Comparison of particle concentration variation with

diameter from coagulation Equation (3.50) and

Smoluchowski Equation (3.52)

Particle concentration variation with diameter at different location for a turbulent plume

Particle concentration variation with diameter in a

laminar plume at different times $\left(\mu=1.79 \mathrm{e}-4\right.$ dynes $\left.\mathrm{sec} / \mathrm{cm}^{2}\right)$

Particle concentration variation with diameter in a turbulent

plume at different times $\left(\mu=5.79 \mathrm{e}-2\right.$ dynes $\left.\mathrm{sec} / \mathrm{cm}^{2}\right)$

Particle concentration variation with diameter at a

location $0.5 \mathrm{~m}$ (20") from the stack outlet by considering

(a) nucleation+coagulation, and (b) nucleation+coagulation +

condensation effects....

Particle concentration variation with diameter at a

locations in a turbulent using nucleation+coagulation+

condensation model

Comparison with experimental data of particle concentration variation with diameter (sulfur fuel level $=13.1 \mathrm{ppm}$, relative humidity $=30 \%$ )

Comparison with experimental data of particle concentration variation with diameter (sulfur fuel level $=125 \mathrm{ppm}$, relative humidity $=30 \%$ ) 154

Particulate matter prediction on different sulfur fuel level (13.1 and $125 \mathrm{ppm})$ at $0.14 \mathrm{sec}(30 \%$ relative humidity, dilution ratio 10 ) 155 
Figure 4.47 Particulate matter prediction on different sulfur fuel level

(13.1 and $125 \mathrm{ppm})$ at $0.34 \mathrm{sec}(30 \%$ relative humidity,

dilution ratio 10) ................................................................. 156

Figure 4.48 Model prediction on particle number concentration of different

sulfur levels (13.1 ppm and $300 \mathrm{ppm})$ at $0.14 \mathrm{sec}(30 \%$ relative

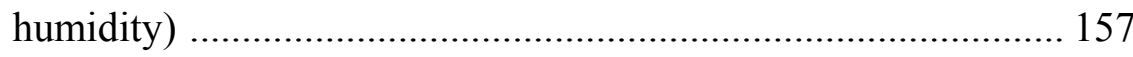

Figure 4.49 Model prediction on particle number concentration of different

relative humidity on $125 \mathrm{ppm}$ sulfur fuel content at $0.17 \mathrm{sec} . . . .158$ 


\section{List of Tables}

Table 2.1 Summary of particle dynamics and transformations and processes........ 65

Table 4.1 Summary of the major findings of this research .............................. 159 


\section{LIST OF SYMBOLS / ABBREVIATIONS/ NOMENCLATURE}
A
- surface area of the nucleus
$\mathrm{A}_{\mathrm{mk}}$
- collection efficiency between m-and k- particle
$\mathrm{B}$
- empirical constant equal to 0.5
$\mathrm{B}_{\mathrm{AV}}$
- average growth rate
$\mathrm{C}$
- time-dependent number concentration $\left(\right.$ No. $\mathrm{cm}^{-3}$ ) of particle of volume
CARB
- California Air Resources Board
CFD
- computational fluid dynamics
CMD
- Count Median Diameter
$\mathrm{C}_{\mathrm{i}}$
- mean concentration of the $i^{\text {th }}$ species
$\mathrm{c}_{\mu}$
- constant of the model
D - diffusivity of the vapor molecules
DR
- Dilution Ratio
$\mathrm{d}_{\mathrm{Nn}}, \mathrm{d}_{\mathrm{Na}}$
- geometric mean particle diameters for nuclei and accumulation modes
EPA
- Environmental Protection Agency
$\Delta \mathrm{G}^{*}$
- free energy required to form an embryo
$\mathrm{g}_{\mathrm{i}}$
- magnitude of the gravitational acceleration in the i-direction
GVWR - gross vehicle weight rating
$\mathrm{h}$
- static enthalpy
$\mathrm{HC}$
- hydrocarbons
$\mathrm{J}$
- integers for J space transformation (Ch. 2)
$\mathrm{J}_{\mathrm{i}, \mathrm{i}}$
- diffusion flux of the $i^{\text {th }}$ species arising due to concentration gradients,
$\mathrm{k}_{\mathrm{b}}$
- Boltzmann's constant
k - turbulent kinetic energy
$\mathrm{K}_{\mathrm{h}}$
- equilibrium constant for formation of hydrate containing $h$ water molecules
Kn $\quad$ - Knudsen number (2N/D)
$\mathrm{k}_{\mathrm{t}}$
- effective conductivity due to turbulent transport $\left(\mathrm{k}_{t}=\mu_{t} / \mathrm{Pr}_{t}\right)$
- total number of particles per unit volume of fluid (Ch. 2) 


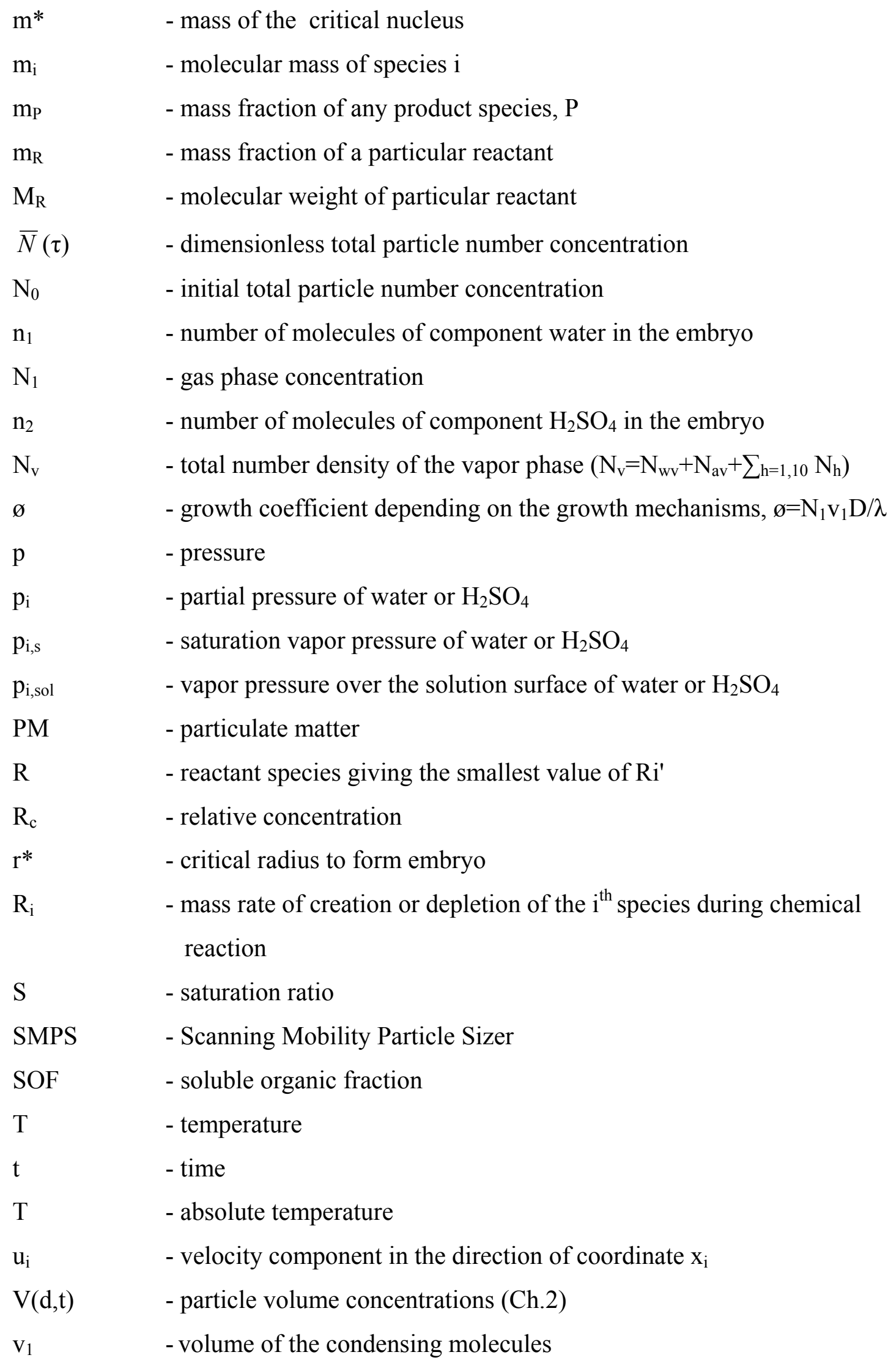




\begin{tabular}{|c|c|}
\hline WVU & - West Virginia University \\
\hline $\mathrm{Z}$ & - Zeldovich nonequilibrium factor \\
\hline$\beta$ & - coagulation kernel $\left(\mathrm{cm}^{3} \mathrm{No}^{-1} \mathrm{~s}^{-1}\right)$ of two colliding particles \\
\hline$\varepsilon$ & - dissipation rate \\
\hline$\eta$ & - viscosity \\
\hline$\theta$ & $\begin{array}{l}\text { - angle between the } \mathrm{n}_{\mathrm{w}} \text { axis and direction of growth at the saddle point } \\
\text { in three-dimensional space }\left(\mathrm{n}_{\mathrm{w}}, \mathrm{n}_{\mathrm{a}}, \Delta \mathrm{G}\right)\end{array}$ \\
\hline$\lambda$ & - mean free path \\
\hline$\mu$ & - laminar viscosity \\
\hline$\mu_{\mathrm{t}}$ & - turbulent viscosity \\
\hline$\mu_{1 \mathrm{~g}}$ & - chemical potentials of components water in the gas phase \\
\hline$\mu_{11}$ & $\begin{array}{l}\text { - chemical potentials of water taken for a macroscopic amount of a } \\
\text { liquid phase of the same composition }\end{array}$ \\
\hline$\mu_{21}$ & $\begin{array}{l}\text { - chemical potentials of } \mathrm{H}_{2} \mathrm{SO}_{4} \text { taken for a macroscopic amount of a } \\
\text { liquid phase of the same composition }\end{array}$ \\
\hline$\mu_{2 g}$ & - chemical potentials of components $\mathrm{H}_{2} \mathrm{SO}_{4}$ in gas phase \\
\hline$v^{\prime \prime} \mathrm{R}, \mathrm{k}$ & - stochiometric coefficient for product \\
\hline$v_{i^{\prime}, k}^{\prime}$ & - stochiometric coefficient for reactant \\
\hline$\xi$ & $\begin{array}{l}\text { - variable of integration which depends on total particle } \\
\text { concentration }\end{array}$ \\
\hline$\sigma$ & - surface tension of the binary mixture \\
\hline$\sigma(\tau)$ & - coefficient accounting for any temporal variation of growth rate \\
\hline$\sigma_{\mathrm{n}}, \sigma_{\mathrm{a}}$ & - standard deviations for nuclei and accumulation modes, respectively \\
\hline$\tau$ & - dimensionless time \\
\hline$\alpha$ & - adjustable parameter \\
\hline$\rho$ & - fluid density \\
\hline$\psi(\mathrm{J}, \mathrm{t})$ & - particle distribution function in J-space transformation \\
\hline
\end{tabular}




\section{CHAPTER 1 \\ INTRODUCTION}

In recent years, there has been an increasing need for models to accurately predict the nucleation, coagulation, and dynamics of particles emitted from diesel-fueled vehicles. The public health community, automotive industry and academia have focused their attention on developing sampling and measurement techniques that will enable a better understanding of the origin and fate of combustion generated particulate matter.

\subsection{OVERVIEW}

The mass concentration of particulate matter (PM) emitted from the diesel engines has been decreasing steadily over the past 20 years mainly due to the advancement of engine technology and improvement in fuel quality. However, knowledge of particle number concentration, which is strongly influenced by ultra fine particle $\left(\mathrm{d}_{\mathrm{p}}<100 \mathrm{~nm}\right)$ formation during dilution, is very limited. Current regulations for mobile source particulate matter emissions are based upon total particulate mass concentration. However, size, number and surface area of PM to which an individual is exposed to may be more relevant than their mass. For assessment of PM health effects, and the fact that ultra fine particles contribute more to number than mass emissions, it is important to identify both mass, size distributions and concentrations of particulate matter emitted by engines.

Diesel engines are typically used in many on- and off-road applications. Most of these applications are subjected to exhaust emission regulations based on environmental 
and health concerns related in part to the particles found in diesel exhaust. After decades of focusing attention on visible emission, mass emissions, and chemical composition, the industry and regulatory bodies have recently focused their attention on another important characteristic of particulate matter (PM) emissions, that is, particle size. It has been known for some time that respiratory systems function much like multistage impactors, collect the largest particles near the inlet (the nose), and leaving the smallest particles to deposit in the deepest parts (the air sacs of the lungs) (Lippmann, 1976). It has also been known for some time that diesel exhaust might contain large quantities of small nanoparticles (Kittelson et al., 1978). Most often, these observations were primarily qualitative, and were used to evaluate the effect of these small particles on the environment. However, the current state-of-the-art models allow computational fluid dynamics (CFD) analysts to predict the size and concentration of these small nano-sized particles.

Some recent studies (for example, Ahlvik et al., 1998; Boyce et al., 2000; Gautam et al., 2000) have shown that the diesel PM remains in a state of continuous transformation (unstable) for some time after it is emitted into the atmosphere. This is in part due to the continuation of in-stack coagulation and adsorption, but also due to the significant quantities of condensable organics and inorganics usually present in diesel exhaust.

The fate of these condensable organics/inorganics is significantly affected by the dilution and atmospheric aging of the exhaust stream. A number of processes occur 
during atmospheric aging that can alter the size distribution of an aerosol, including homogeneous nucleation, binary homogeneous nucleation and coagulation. Homogeneous nucleation is the spontaneous formation of a nuclei or nano-sized particles (nano-particles) from volatile material within a locally supersaturated zone. Binary homogeneous nucleation involves the same volatile material and driving force (that is, saturation), but also needs "seed" nuclei that considerably lower the required degree of saturation. Saturation is the condition at which the partial pressure of a volatile material equals its vapor pressure; degree of saturation is usually expressed as a ratio of these pressures. After hot exhaust is released into the air, the partial pressure of its volatile components decreases with dilution. The vapor pressure of these components is a function of temperature, which also decreases with dilution. Since the vapor pressureversus-temperature relationship is nonlinear, it is normal for the saturation ratio to reach a maximum at some dilution level. The organic fraction of diesel exhaust reaches its maximum degree of saturation somewhere between a 5:1 and 50:1 dilution ratio, but does not usually achieve the necessary super-saturation required for homogeneous nucleation (Friedlander, 1977). On the other hand, $\mathrm{H}_{2} \mathrm{SO}_{4}$ may reach the super saturation levels necessary for homogeneous nucleation at ratios between 10:1 and 50:1, possibly serving as "seed" nuclei for binary homogeneous nucleation (Baumgard and Johnson, 1996).

\subsection{OBJECTIVES OF THIS RESEARCH}

The main objectives of this research are to investigate the formation of nucleation and coagulation modes of particulate matter in the exhaust plume of a class- 8 tractor truck powered by a 330 horsepower Cummins M11 electronically controlled diesel 
engine; and to develop a numerical model for predicting the particle size distribution in the nucleation and coagulation modes. The uniqueness of the approach in this research efforts particulate matter (PM) size distribution data is not required a priori to solve the nucleation/coagulation equations; instead the PM concentration is predicted based on the fuel sulfur content, fuel to air ratio, exhaust flow rate and the ambient conditions.

A commercially available CFD software FLUENT ${ }^{\mathrm{TM}}$, was used to predict the variations of carbon dioxide concentration inside a turbulent plume created in NASA Langley aircraft testing wind tunnel, via an eddy dissipation model. Additionally, these computational models were validated with the available experimental data collected by WVU researchers under a Coordinating Research Council sponsored program (CRC E-43 study; Boyce et al., 2000, Gautam et al., 2000). This work also shows the benefits of CFD modeling in applications where dispersion correlations are not required a priori, instead the dispersion coefficients are calculated by solving the turbulent kinetic energy and dissipation equations.

The research objectives were accomplished through the completion of the following tasks:

- Performed a thorough literature search to understand the behavior and interaction of aerosols in diesel engine exhaust's plume.

- Used advanced computational fluid dynamics (CFD) concepts and tools to model the dispersion of a turbulent plume emitted by a heavy-duty tractor truck traveling at 55 mph in the absence of any cross winds. 
- Employed FLUENT ${ }^{\mathrm{TM}}$ software to formulate the physical phenomena important to the plume dispersion, including the effects of turbulent mixing, convection, diffusion, temperature variations, and species transport among others.

- Determined the relative concentration of $\mathrm{CO}_{2}$, dilution ratio, and temperature variations inside the plume using a CFD model with detailed truck geometry

- Validated the above predictions with the experimental data obtained from the wind tunnel tests.

- Compared the above parameters predicted from a detailed CFD model with the traditionally used Gaussian Dispersion Models.

- Refined an existing binary nucleation theory in the literature for $\left(\mathrm{H}_{2} \mathrm{SO}_{4}-\mathrm{H}_{2} \mathrm{O}\right)$ nucleimode particle formation in the exhaust plume.

- Used the above mentioned refined model to predict the conditions that are necessary in the formation of nuclei particles. Used these results as a basis for formulating an empirical model of predicting nuclei mode particles.

- Incorporated the effects of physical and chemical phenomena such as hydration and coagulation on particulate matter that occur in the dilution tunnel or exhaust system.

- Developed a FORTRAN code to compute the effective particle diameter sizes (and distribution) in the nucleation and coagulation modes, and validated this code with the available experimental data.

- Integrated the coagulation model to include the dilution ratio effects in the wind tunnel. Developed the numerical algorithm, which contained the effects of nucleation and coagulation modes simultaneously. 


\subsection{TECHNICAL APPROACH TO ACCOMPLISH ABOVE OBJECTIVES}

The above objectives were accomplished in three phases as described below:

Phase-I: $\quad$ The first phase of this research was focused on determining concentration of $\mathrm{CO}_{2}$, dilution ratio, and temperature variations inside the plume of a heavyduty diesel truck operating at $55 \mathrm{mph}(88.51 \mathrm{~km} / \mathrm{hr})$ using a Computational Fluid Dynamics (CFD) model. The advanced CFD technique is utilized to accurately predict the variation of carbon dioxide concentration, dilution ratio, and temperature variations inside a turbulent plume using a $\mathrm{k}-\varepsilon$ eddy dissipation model. A detailed geometry of the truck including tires, cabin, wind-deflector, and the exhaust stack was created using Gambit ${ }^{\mathrm{TM}}$ software. The above geometry was discretized with approximately 500,000 grid cells/control volumes, and solved using a Fluent ${ }^{\mathrm{TM}}$ segregated solver. Additionally, the species concentrations inside the plume were determined using a probability density function (PDF) mixture fraction formulation and the modified Gaussian model.

Phase-II: $\quad$ The second phase involved the mathematical formulation of coagulation kernels, and refinement of a coagulation model to account for the effect of dilution ratio on coagulation of particulate matter that occurs in the wind tunnel. The dilution ratio at different locations inside the tunnel determined in Phase-I was used. The integro-differential formulation of the poly-disperse coagulation equation was solved for time dependent particulate matter concentration using semi-implicit finite difference scheme. A FORTRAN code was developed to solve the above equation. In this phase, PM concentration obtained in the wind 
tunnel study at the measurement location closest to the plume source was used as an initial condition. The above model was then used to predict the PM concentration variation at various locations in the tunnel.

Phase-III: The last phase of this research was dedicated to include the effects of nucleation and coagulation on particulate matter formation, simultaneously. This was achieved by refining the existing binary nucleation theory in the literature for $\left(\mathrm{H}_{2} \mathrm{SO}_{4}-\mathrm{H}_{2} \mathrm{O}\right)$ nuclei-mode particle formation in the exhaust plume. This equation was solved from first principles using a finite-difference scheme. Incorporating nuclei mode in the exhaust stack did not require the initial condition that was used in Phase-II. The nuclei number concentration was determined directly from fuel sulfur concentration. No attempt was made to incorporate the distribution of lube oil ash to nuclei mode particles, primarily due to absence of any experimental data. With this method, PM concentration and size distribution data was not required a priori to solve these equations; instead the particulate matter concentration was predicted based on the fuel sulfur content, fuel to air ratio, exhaust flow rate and ambient conditions.

A detailed survey of various models available in the literature, including various statistical, empirical, and CFD plume submodels are described in Chapter 2. Chapter 2 also includes a discussion on the effects of nucleation and coagulation modes on the formation of particulate matter. The literature review is followed by Theoretical 
Formulations in Chapter 3. The results are presented in Chapter 4. A road map to future work along with conclusions is presented in Chapter 5 . 


\section{CHAPTER 2}

\section{LITERATURE REVIEW}

In order to evaluate the impact of mobile source exhaust emissions on the environment, it has become increasingly important that the dispersion of pollutants be predicted accurately. Recently, a great deal of attention has been focused on the issue of particulate emissions from internal combustion engines. Particulate emissions from internal combustion engines are a significant source of primary particles smaller than 2.5 $\mu \mathrm{m}\left(\mathrm{PM}_{2.5}\right)$ in urban areas (Pope et al., 2002; Lighty et al., 2000; Graskow et al., 1998; Morgan et al., 1997). In particular, there is a great deal of concern relating to the health effects of nano-particles, which are less than 50 nano-meters in diameter. Because of their extremely small size, nano-particles are able to penetrate deep into lungs where they may enter interstitial tissues, causing severe respiratory inflammation, acute pulmonary toxicity and other health hazards (Pope et al., 2002; Seaton et al, 1995).

\subsection{HEALTH HAZARDS OF DIESEL EXHAUST}

Donaldson et al. (1998) described the non-ultrafine particles, which can produce an efficient mechanism for the removal of particles from the lung (unless this is prevented by harmful particles such as quartz or asbestos). In comparison with fine particles, an equal mass of ultrafine particles contains thousands of times more particles, which means that the macrophages must be highly efficient to remove all of the ultrafine particles that deposit in the alveolar regions of the lung (for example, see Figure 2.1). Macrophages (a mobile cell which moves around the surface of alveolar region) seem not to be able to remove ultrafine particles as efficiently as larger particles and in any 
case, the high number of particles may present an insurmountable task for the macrophages. Finally, the ultrafine particles may even damage macrophage cell functions although the mechanism by which the ultrafine particles damage macrophages remains unclear. Ultrafine particles is defined as particulate matter (PM) less than $100 \mathrm{~nm}$ diameter and nano-particle as PM less than $50 \mathrm{~nm}$ in diameter.

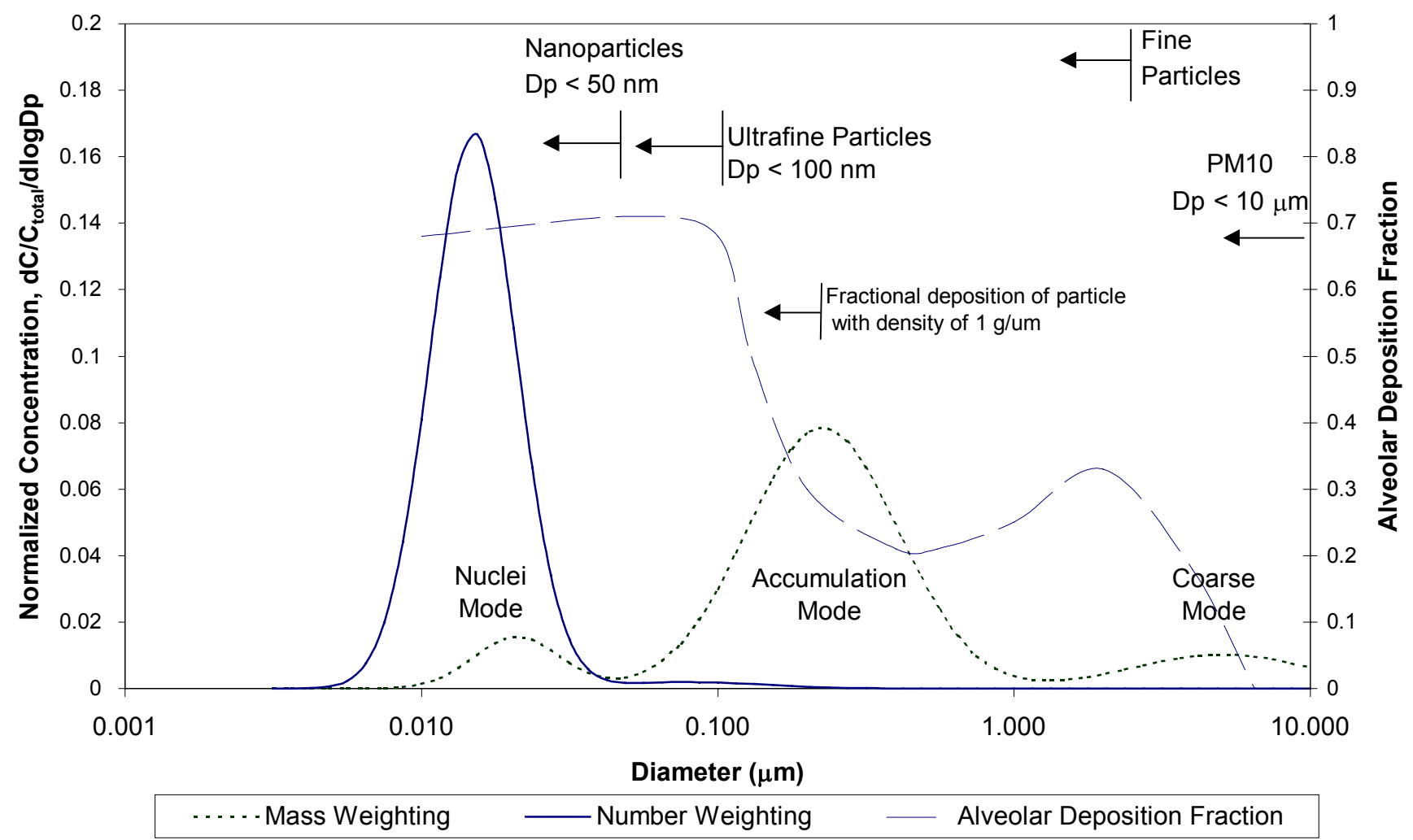

Figure 2.1 Engine exhaust mass and number weighted size distributions shown with alveolar deposition (Kittelson, 1998) 
Recently, Constantini (2000) showed an association between exposure to ambient particulate matter $(\mathrm{PM})$ less than $10 \mu \mathrm{m}$ in diameter $\left(\mathrm{PM}_{10}\right)$ and less than $2.5 \mu \mathrm{m}$ in diameter (PM2.5) and increased mortaility and morbidity. One of the reasons that ultrafine particles are thought to be of special importance is that the alveolar deposition fraction is increasing dramatically for particles with a diameter from 0.1 to $0.01 \mu \mathrm{m}$. Constantini (2000) concluded that transition metals may be involved in some of the effects because of their ability to catalyze redox reactions in lung cells leading to the production of reactive oxygen species and increased production of inflammatory mediators.

Recently, special concerns have been raised regarding particles in the ultrafine and nano particle diameter range. Particles that are non-toxic in a size range greater than $100 \mathrm{~nm}$ may be toxic in range less than $100 \mathrm{~nm}$. It was observed by Seaton et al. (1995) that exposure of $0.02 \mu \mathrm{m}$ diameter $\mathrm{TiO}_{2}$ retained more nano-particles in the interstitial tissue of the lung and developed marked inflammatory response than if $0.25 \mu \mathrm{m}$ diameter particles were used in their study. Comparison of surface free radical activity of ambient $\mathrm{PM}_{10}$ particles, and $0.5 \mu \mathrm{m}$ and $0.025 \mu \mathrm{m}$ diameter $\mathrm{TiO}_{2}$ showed significant activity for $\mathrm{PM}_{10}$, and much greater activity for 0.025 than for $0.5 \mu \mathrm{m} \mathrm{TiO}{ }_{2}$ (Donaldson et al., 1996). It is striking that $\mathrm{TiO}_{2}$, usually regarded as biologically inert, produces strong responses in the form of nano-particles. Another normally inert substance, Teflon, showed strong adverse health effects when administered in the form of nano-particles. Modest concentrations of $0.03 \mu \mathrm{m}$ Teflon fume particles were shown to cause acute pulmonary toxicity (Warheit et al., 1990). 
Diesel PM may be emitted in size ranging from $0.01 \mu \mathrm{m}$ to $300 \mu \mathrm{m}$, and is known to have soluble organic fraction (partially burned fuel and lube oil components) adsorbed on the particle surface. The soluble organic fraction (SOF) contains poly-cyclic aromatic hydrocarbons (PAH) and nitro-PAHs that are known to be carcinogenic. Diesel PM formation (nucleation, condensation, and coagulation) is highly dependent upon postcombination distribution/mixing processes. Characteristics of diesel PM are discussed in section 2.3. In order to evaluate the impact of pollutants and nano-particles emitted from the exhaust of diesel engines on the environment, it has become increasingly important that the dispersion of pollutants/particles be predicted accurately. Modeling pollutant transport and the PM size distribution in the ambient is complicated by the fact that there are many turbulent mixing time scales and spatial scales present which directly influence the dispersion of the exhaust plume.

\subsection{PLUME MODELS}

The traditional approach to predicting pollutant dispersion in the atmosphere is the use of Gaussian plume models. The Gaussian models are based on a steady state assumption, and they require the flow to be in a homogeneous and stationary turbulence state (Zannetti, 1990). Hence, these models can not be applied with much validity or confidence to applications where inhomogeneous effects, for example dissipating turbulent eddies near physical walls are present. It should be noted that Gautam et al. (2000) and Kim et al. (2001) showed a fairly good agreement in far field regions with experimental data on $\mathrm{CO}_{2}$ concentrations in a heavy duty truck plume in the NASA Langley aircraft testing wind tunnel (Boyce et al., 2000, Gautam et al., 2000). Using the 
Pasquill-Gifford dispersion equation, the Gaussian model was modified, and then extended into the near field (Gautam et al., 2000; Kim et al., 2002). It was envisaged that in such situations, rather than attempting an empirical modification of Gaussian model, a more fundamental approach of finding a numerical solution to the governing partial differential equations of conservation of mass, momentum, energy and species was warranted.

Heavy-duty diesel vehicle emissions of particulate matter (PM), in general, and PM size distribution and concentrations in particular, are of eminent concern. It is imperative that the exhaust plume characteristics and history, and its response to the local flow and temperature fields be known under representative speed and load conditions. The time and spatial scaling associated with gas-to-particle transformations and particle growth are small; hence a near-field plume study becomes important. To this end, a solution of an Eulerian advection-diffusion equation with the k- $\varepsilon$ turbulence closure is proposed in the present investigation.

Over the years, technical development and growth of scientific understanding has greatly accelerated in all types of empirical, analytical and numerical models to predict the concentration variations in a dispersion plume. These models range from the empirical Gaussian distribution models to highly sophisticated large eddy simulation models. In general, these models may be divided into following categories described briefly in the subsequent sections:

(i) Empirical Gaussian models (Hanna, 1984; Kaharabata et al., 2000) 
(ii) Similarity models (Sawford, 1983; Huai and Li, 1993; Obasaju and Robins, 1998)

(iii) Probability Density Function (PDF) models (Weil et al., 1997; Yee and Chan, 1997; Ferrero and Anfossi 1998; Reynolds, 2000)

(iv) Statistical models (Durbin, 1980; Lamb, 1982; Sawford, 1983; Heinz and vanDop, 1999)

(v) k- $-\varepsilon$ models (Hwang and Chiang, 1988; Sharan and Yadav, 1998)

(vi) Large eddy simulation models (Sykes et al., 1984; Heinz and vanDop, 1999)

\subsubsection{Empirical Gaussian Models}

The Gaussian plume models, the simplest of the dispersion models, are based on the analytical solution of the transport of species advection-diffusion equation by assuming constant diffusion coefficients. The principal framework of empirical equations in Gaussian plume models is based on the assumption that concentrations from a continuously emitting plume are proportional to the emission rate, and that these concentrations are diluted by the wind. In its simplest form, Gaussian model assumes that the pollutant does not undergo chemical reactions or other removal processes in traveling away from the source. This form assumes that the plume spread has a Gaussian distribution, the wind affecting the plume is uniform, and the plume is perfectly reflected at the surface. Many applications of this equation to environmental studies are reported in the literature (Turner, 1970; Pasquill, 1974). 
The diffusion coefficients are correlated empirically by taking into account the Pasquill-Gifford stability classes (A to F) which range from extremely unstable atmospheric conditions to moderately stable conditions (Gifford, 1961). Application of these models is limited to some idealized meteorological conditions. The Gaussian plume equation for estimating normalized ground-level concentration is typically of the form (Hanna, 1984; Kaharabata et al., 2000):

$$
\frac{C U}{Q}=\frac{\exp \left[-1 / 2\left(y / \sigma_{y}\right)^{2} \exp \left[-1 / 2\left(h / \sigma_{z}\right)^{2}\right.\right.}{\pi \sigma_{y} \sigma_{z}} m^{-2}
$$

where $\mathrm{C}$ is pollutant concentration $\left(\mathrm{g} / \mathrm{m}^{3}\right), \mathrm{U}$ is mean wind speed affecting the plume $(\mathrm{m} / \mathrm{s}), \mathrm{Q}$ is emission rate $(\mathrm{g} / \mathrm{s}), \mathrm{h}$ is effective emission height above the ground $(\mathrm{m}), \sigma_{\mathrm{y}}$, and $\sigma_{\mathrm{z}}$ are the values of the horizontal (y) and vertical (z) dispersion coefficients (m), respectively. It is apparent from Equation (2.1) that values of $\sigma_{\mathrm{y}}$ and $\sigma_{\mathrm{z}}$ are required $a$ priori. Values of $\sigma_{\mathrm{y}}$ and $\sigma_{\mathrm{z}}$ are normally constants chosen from some empirical correlations depending on the stability classes defined above. Gautam et al. (2000) showed that a simple Gaussian predictor of plume concentrations was in reasonably good agreement with measurements in the far field region, provided that diffusion coefficients, expressed as a function of ambient stratification were adjusted. It is well known that the Gaussian models, in general, may not provide accurate solutions near physical obstacles where the dispersion of eddies is controlled by local turbulence. The Gaussian predictor (Gautam et al., 2000) was developed to provide researchers with a much-needed tool for a quick estimation of dilution ratios in the exhaust plume of a heavy-duty vehicle under on-road operating conditions. 
Huber (1991) investigated the relationship between Gaussian plume models and wind tunnel models. Wind tunnel measurements of the distribution of tracer concentrations downwind of a point source in the near wake of a rectangular model building were evaluated. The differences in observed velocity and concentrations between the results for the low-turbulence and simulated atmospheric boundary layer flow were not significant far away from the buildings, but these differences increased in significance in the vicinity of buildings. The application of fluid models to characterize the initial building wake dispersion and the virtual source technique into a Gaussian plume model as demonstrated in this study, should be valid for a wide range of situations in the vicinity of physical obstacles or buildings.

\subsubsection{Similarity Models}

Similarity models for concentration fluctuations have a potential to provide the key to a generalized model for dispersion phenomenon in case of a plume rise and diffusion modeling. Similarity models are presently not well developed; probably due to insufficient field data that could be used to derive generalized dimensionless relationships (Huai and Li, 1993; Obasaju and Robins, 1998). An initial attempt at explaining concentration fluctuations through similarity theory was made by Sawford (1983) using statistical models. It was inferred by the above authors that at large times or distances, the fluctuations approach a constant dependent only on source size $\left(\sigma_{0}\right)$, and Eulerian length scale, (L) as $\propto\left(\mathrm{L} / \sigma_{\mathrm{o}}\right)^{0.3}$. This relation provides only one sample approach, but further extensive laboratory and field data will be required to develop advanced similarity groups 
before this technique could be used in the practical applications with any degree of confidence.

\subsubsection{Probability Density Function Models}

This class of models is founded on the concept that the probability density function (PDF) of concentration fluctuations is directly related to the probability density function (PDF) of turbulent velocity fluctuations. In this sense, the so-called PDF models are very similar to Gifford's (1959) meandering plume model. Since, it is assumed in the PDF theory that individual air parcels follow straight-line trajectories, these techniques are mostly valid for travel times less than the Lagrangian integral (diffusion or mixing) time scale of the turbulence. This type of modeling technique is generally applied to estimate the ground level concentrations due to releases from tall stacks in convective conditions (Weil et al., 1997; Yee and Chan, 1997; Ferrero and Anfossi, 1998; Reynolds, 2000; Kim et al., 2002).

In the probability density function (PDF) approach, the concentration fluctuations inside the plume are directly related to the PDF of turbulent velocity fluctuations. This technique is mostly suitable for travel times less than the Lagrangian integral time scale of the turbulence. Furthermore, this theory should be applied in situations where the averaging times are larger than the integral time scale. A large number of mathematical forms have been used in the literature to model the concentration PDF shape, for example, the log normal distribution (Csanady, 1973), the exponential distribution 
(Hanna, 1984), the clipped-normal distribution (Lewellen and Sykes, 1986), the betaJacobi distribution (Derksen and Sullivan, 1990) among others.

\subsubsection{Statistical Models}

Statistical models, similar to Monte Carlo simulations, are based on the use of a computer to follow the Lagrangian trajectories of thousands of particles from which the concentration PDF can be calculated at a given location. The statistical theory is based on the actual velocities of individual particles in a steady homogeneous turbulence field (Durbin, 1980). Under this assumption, the statistics of the motion of one typical or sample particle provides a statistical estimate on the behavior of group of particles in the flow domain. The statistics obtained using two particles simultaneously provide an estimate of the behavior of a cluster of particles (Durbin, 1980, Lamb, 1982, Sawford, 1983). Gifford (1982) show that relative diffusion can also be realistically estimated for small travel times through use of single particle Monte Carlo models. The statistical theory is more suitable for describing the spread of a plume in the crosswind direction regardless of the height. However, for vertical spread it is suitable only in the early stages of travel from a source that is considerably elevated above the ground. It is apparent that the crosswind component of turbulence in the atmosphere is nearly homogeneous since the variations in the scale and intensity of crosswind velocity with height are often small. On the other hand, the vertical wind velocity component is decidedly non-homogeneous, since characteristically vertical velocity component increases with height above the

ground. Application of statistical models to the problem of estimating concentration fluctuations have taken place during the last few years, and there are a wide variety of 
modeling approaches available, if tested with the experimental data will provide a viable approach to estimating concentration fluctuations in the future.

\subsection{5. k- $\varepsilon$ models}

The k- $\varepsilon$ models are developed from the conservation of mean-square concentration variations with the assumption that the concentration variance is a transportable quantity, and it can be transferred and dissipated in the same way as the turbulent kinetic energy. Central ingredients in this theory are the use of the kinetic energy equation, together with the requirement that the most dominant Reynolds stress component be self-preserved (Hwang and Chiang, 1988; Sharan and Yadav, 1998). This model is generally suitable to predict in-plume concentration variations in the presence of various obstacles or walls. The governing equations for this model are discussed in detail in Chapter 3.

\subsubsection{Large Eddy Simulation Models}

Large Eddy Simulation (LES) models are very computer intensive but do produce results that are probably closer to in-field conditions. The computational grid size in these models is set much smaller than the dominant eddy size, and the simulations can be run only in time-dependent transient mode (unlike steady state in $\mathrm{k}-\varepsilon$ models). While a model run is equivalent to one field experiment, to construct ensemble statistics (e.g., for concentration fluctuation variance) may require at least ten runs. This is one of the drawbacks of LES models. In the LES technique, large-scale flow features are resolved explicitly on a grid with subgrid-scale features induced by turbulence parameterization 
(Sykes et al., 1984; Heinz and vanDop, 1999). The technique results in the generation of detailed information that would have been difficult or impossible to obtain experimentally in the atmosphere. In essence, an LES run represents a surrogate field experiment under controlled conditions which can provide great insight into the growth and descent of plumes.

After reviewing the different models described in this section, a test case CFD simulation on a pipe 5 inches $(0.127 \mathrm{~m})$ in diameter enclosed in a large enclosure $(127 \mathrm{~m}$ x $127 \mathrm{~m} \times 25 \mathrm{~m}$ ) was conducted using the k- $\varepsilon$ model. It was determined that the $\mathrm{k}-\varepsilon$ turbulent eddy dissipation model captured the exponential decay in $\mathrm{CO}_{2}$ concentration as one moved away from the source, and it also captured the re-circulation of flow near the outlet of the pipe. Also, in the absence of any swirling flow, it was determined that the k$\varepsilon$ turbulent closure was adequate for the present application. The governing equations for the k- $\varepsilon$ model are presented and discussed in the Chapter 3. Once the choice of a turbulence model was made, the next step was to include the effects of nucleation and coagulation modes on the formation of particulate matter, which are described in brief in Sections 2.5. and 2.6. Several researchers (Brown et al., 2000; Boyce et al., 2000) have investigated the aerosol formation in diesel engine exhaust under atmospheric conditions. The next section briefly explains the characteristics of diesel particulate and fundamentals of gas-to-particle transformations and growth of particles, namely nucleation and coagulation. 


\subsection{CHARACTERISTICS OF DIESEL PARTICULATE MATTER}

Diesel exhaust particles are mostly sub-micrometer agglomerates of carbonaceous spherical particles ranging from 10 to $80 \mathrm{~nm}$. Larger particles contain up to 4,000 individual spherical particles clustered as agglomerates up to $3 \mu \mathrm{m}$ (Morawska et al., 1998; Kittelson et al.,1978). A majority of diesel emission particulates have aerodynamic diameters smaller than $1 \mu \mathrm{m}$. These particles are primarily elemental carbon, but also contain adsorbed or condensed hydrocarbons, hydrocarbon derivatives, sulfur compounds, and other materials. Solvent extractable organic components of diesel aerosols represent $5-40 \%$ of the particle mass depending upon the fuel type, engine technology, engine operating conditions, such as, engine speed and torque, and exhaust after treatment systems.

Diesel particle size distributions may be bi-modal with a nuclei mode $(0.0075$ to $0.042 \mu \mathrm{m}$ in diameter) and an accumulation mode (0.042 to $1.0 \mu \mathrm{m}$ in diameter) (Baumgard and Johnson, 1996; Gautam et al., 2000). Figure 2.2. shows the typical diesel PM size distributions and concentrations acquired from heavy-duty vehicles operating on the WVU chassis dynamometer based transportable heavy-duty vehicle emissions testing laboratory (Gautam and Mehta, 2001). The test vehicles were 1998 NOVA transit buses that were powered by Cummins M-11 engines operating on federal diesel No. 2. The vehicles were equipped with a catalytic converter (Model 28277). All of the transit buses had a gross vehicle weight rating (GVWR) of 39,500 lbs (17,916 kg). PM size distributions and concentration measurement were made under steady state operation (20 mph) using a Scanning Mobility Particle Sizer (SMPS, Model CPL 2971). The raw 
exhaust sample was conditioned using WVU's mass flow controllers based mini-dilution tunnel, which was operated to yield a dilution ratio of about 20 . The nuclei mode usually contains $1-20 \%$ of the particle mass and more than $90 \%$ of the particle number.

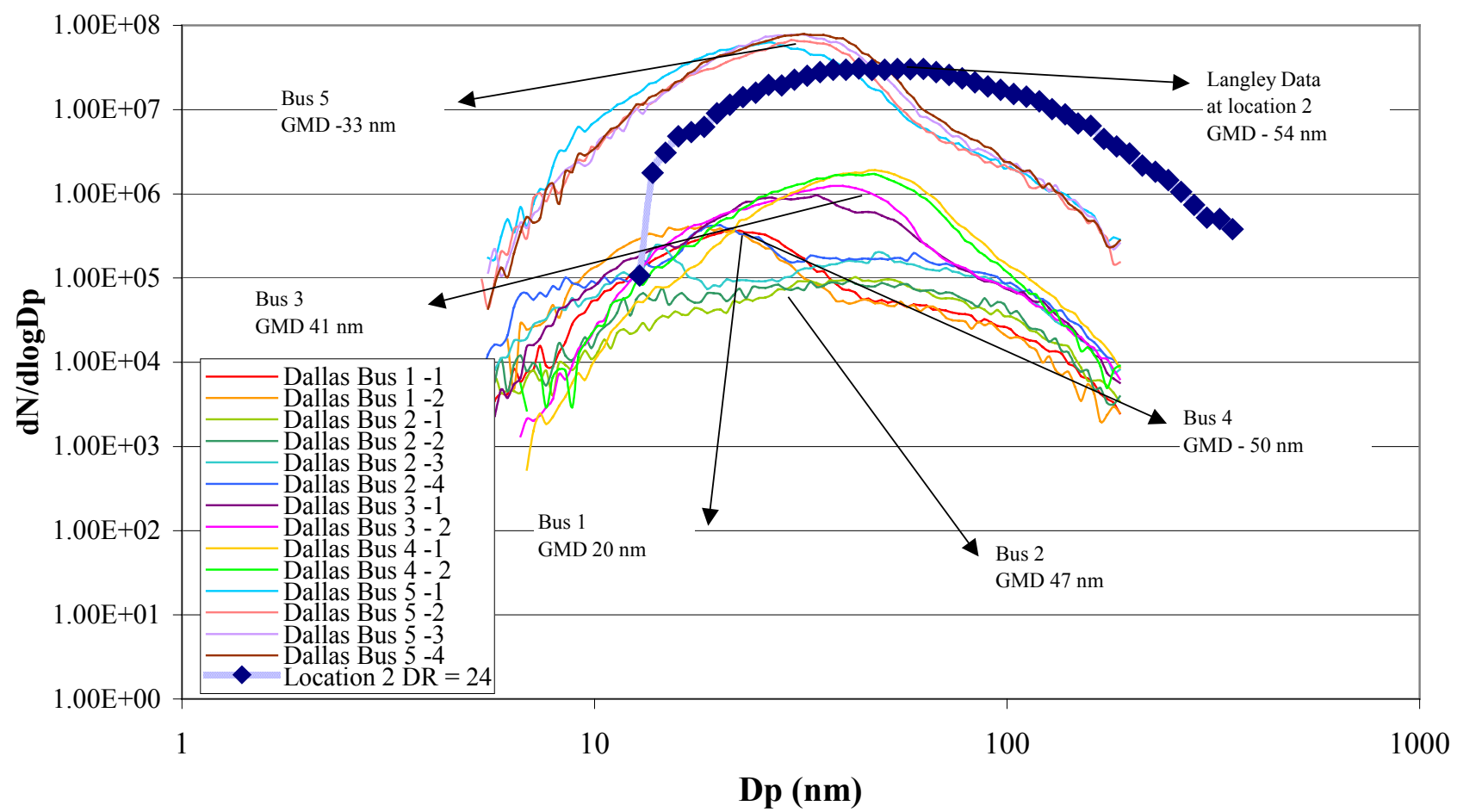

Figure 2.2. Typical engine exhaust particle size distribution for different buses at different locations (Gautam and Mehta, 2001) 
A schematic of the particle formation processes and factors affecting the particle size distributions from the time of combustion to discharge into the atmosphere is shown in Figure 2.3. Primary carbon spherules are formed in the combustion chamber by nucleation of carbon particles onto precursor particles (Heywood, 1988). Once these particles are formed, a large percentage is oxidized during the expansion stroke (Luo et al., 1989). The particles that survive oxidation typically agglomerate to form the long chain aggregates or clusters associated with diesel particles (Baumgard and Johnson, 1992). These particles make up the majority of the accumulation mode. The primary carbon particles that do not agglomerate remain in the nuclei mode size range. Nuclei mode also consists of secondary aerosol formed from nucleation of low volatility vapor in process known as homogeneous nucleation. Experimental observations at Michigan Technological University with a 1988 heavy-duty-diesel engine have indicated that there are major differences in the nuclei-mode particles using ultra low sulfur fuel $(0.01 \mathrm{wt} . \%)$, and federal on- highway diesel fuel with $0.32 \%$ (wt) sulfur (Baumgard and Johnson, 1996).

Exhaust after treatment control technologies, such as a ceramic particle trap, are being used to physically remove the diesel particles from the exhaust stream. Oxidation catalytic converters (OCC) can also be used to change the gaseous species by oxidizing gaseous $\mathrm{HC}, \mathrm{CO}$ and sulfur dioxide $\left(\mathrm{SO}_{2}\right)$. Measuring particle size distributions and concentrations of before and after the exhaust aftertreatment control technology provides information related to post-combustion the physical or chemical particle processes within 


\author{
Particle Formation by \\ Nucleation \\ Oxidation \\ Agglomeration
}

Oxidation

Agglomeration

Thermophoresis
Physical Removal
of particles

Chemical Reactions

of gas phase species

Adsorption and Condensation Nucleation, Gas-to particle Chemical Reactions

Partitioning of HC species between particle and vapor phase

Photochemical Reactions Particle Surface Reactions Gas-to-particle Conversions Partitioning of HC species between particle and vapor phase

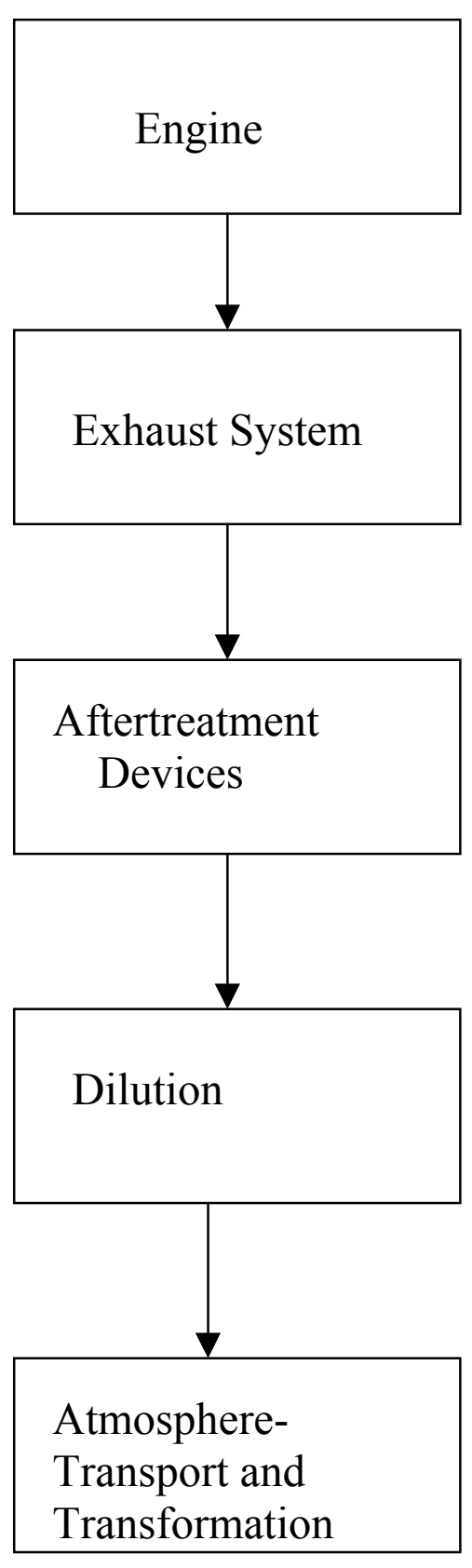

Engine design-fuel injection and mixing

Operating conditions

Fuel

State of Maintenance

Exhaust temperature

Particle Concentration

Exhaust system Design

Ceramic Particle Trap

Oxidation Catalytic Converter

Dilution Ratio

Vapor Pressure

Relative humidity/water vapor concentration

Temperature

Time

Gas species concentrations

Solar Radiation

Time

Figure 2.3. Schematic of the particle and gas phase processes and factors affecting the particle size distributions and chemical composition of the particles from the time of combustion to discharge into the atmosphere, followed by atmospheric transport and transformation (Baumgard and Johnson, 1996) 
the exhaust system. The final particle formation and growth processes take place in the atmosphere. These are mainly photochemical reactions and to a lesser extent particle surface reactions. In addition, gas-to-particle conversions do occur due to the nucleation of hydrocarbons, or oxides of nitrogen, or oxides of sulfur. These particles are considered secondary particles because they were in the gas phase when emitted from the combustion source and converted to particles in the atmosphere. In the dilution process (which approximates short term atmospheric conditions), there is considerable $\mathrm{HC}$ adsorption and condensation occurring onto the particles. The HCs that adsorb onto the particles are associated with the soluble organic fraction. Partitioning of the HC species between the particle and vapor phases also occurs during dilution process. New particles may be formed during dilution process by gas-to-particle processes referred to as nucleation. The details of nucleation process are described in section 2.5.

A few recent studies (Kittelson et al., 1998; Brown et al., 2000) reported tractortrailer plume dilution. Some of the data were collected with the laboratory driven behind the truck, and some were collected with the laboratory riding on the flatbed. The data collected for 50-55 mph roadway conditions suggest two distinct stages of dilution. From the time it is released, the raw exhaust (dilution ratio=1) steadily dilutes over the course of the next $10 \mathrm{~m}(\sim 420 \mathrm{msec})$ to a ratio of 100:1. At some point between $10 \mathrm{~m}$ and $23 \mathrm{~m}$ ( $\sim 1000 \mathrm{msec})$, the exhaust plume is disturbed by the vehicle wake, bringing about a rapid 3:1 dilution beyond what was already taking place. After that, the plume continues to dilute at a rate only slightly slower than what was occurring above the flatbed. Boyce et 
al. (2000) presented dilution ratio data that was measured in the plume of a heavy-duty truck under controlled wind tunnel conditions.

Diesel aerosol consists mainly of highly agglomerated solid carbonaceous material and ash, and volatile organic and sulfur compounds. The structure is illustrated schematically in Figure 2.4. Solid carbon is formed during combustion in locally fuel rich regions. Much of the carbon is subsequently oxidized with the residue exhausted in the form of solid agglomerates. A tiny fraction of the fuel is atomized and evaporated lube oil escape oxidation and appear as volatile or soluble organic compounds (generally described as the soluble organic fraction, SOF) in the exhaust. The SOF contains polycyclic aromatic compounds containing carbon, hydrogen, oxygen, nitrogen, and sulfur. Most of the sulfur in the fuel is oxidized to $\mathrm{SO}_{2}$, but a small fraction is oxidized to $\mathrm{SO}_{3}$ that leads to sulfuric acid and sulfate aerosol. Metal compounds in the fuel and lube oil contribute to inorganic ash in the exhaust gas. The other sources of metalic ash in the exhaust of diesel engines are fuel additives and catalysts from catalyzed exhaust after treatment devices.

Most of the particle size distribution and concentration data currently used by researchers has typically been collected using a Scanning Mobility Particle Sizer (SMPS). A brief overview of principles of operation of an SMPS and sampling techniques is provided in the next section. 
Solid Carbonaceous/Ash Particles with Adsorbed

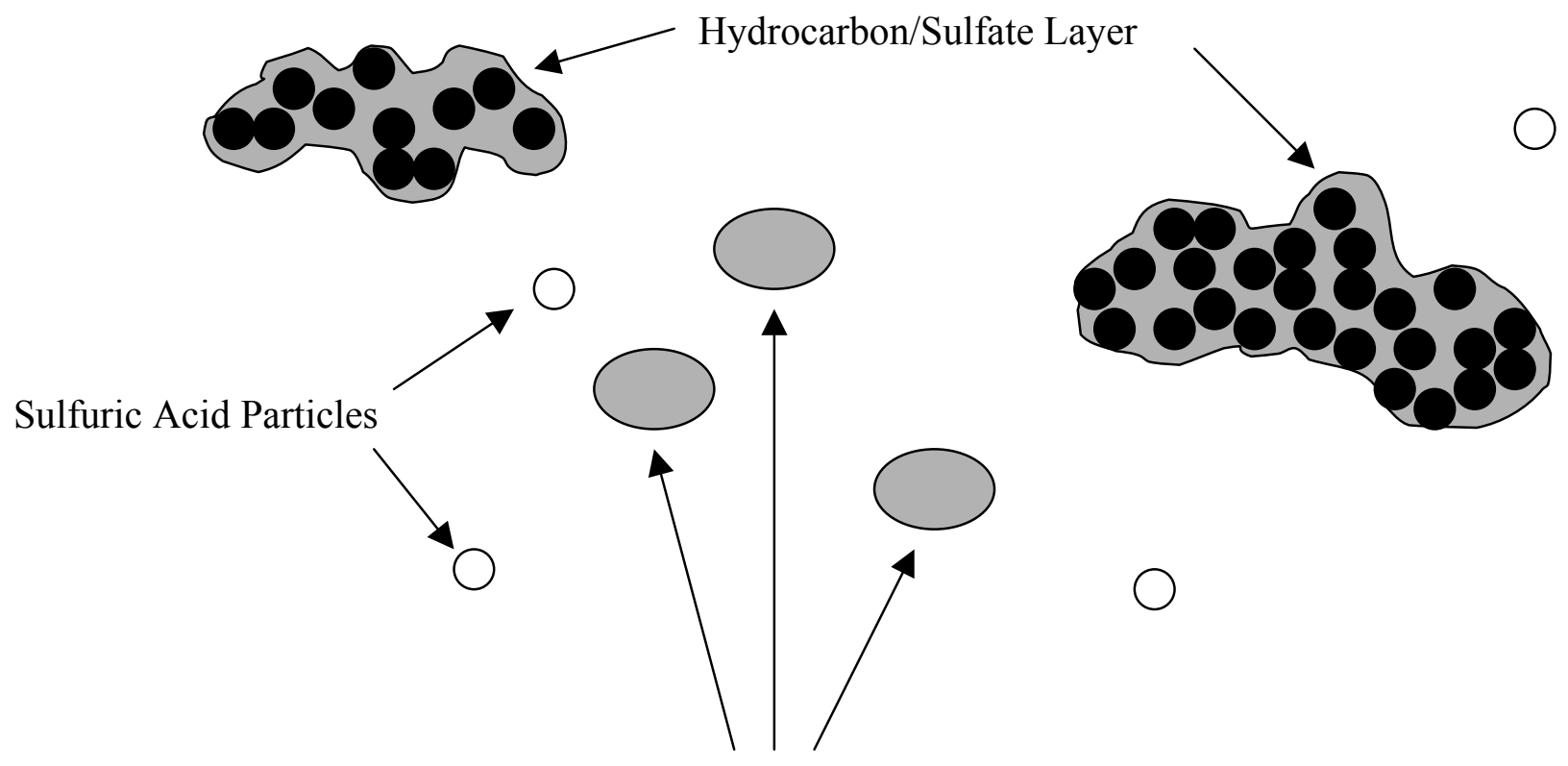

Hydrocarbon/Sulfate Particles

Figure 2.4. Typical structure of engine exhaust particles (Kittelson, 1998) 


\subsection{OVERVIEW OF SMPS AND PM SAMPLING TECHNIQUES}

The scanning mobility particle sizer (SMPS) works on the principal of electrical mobility and is used to measure particle size distributions and concentrations (Graskow et al., 1998). The diluted exhaust sample is first passed through a bipolar ion neutralizer in order to bring charge levels to a Boltzman equilibrium charge distribution. The polydisperse aerosol is then passed into the classification column, which consists of a highvoltage central rod concentrically surrounded by a grounded outer cylinder. Particles are either attracted or repelled by the central rod at different rates, depending on the electrical mobility of the individual particles (electrical mobility depends on the particle's electrical charge and aerodynamic diameter). Particles having the proper electrical mobility exit through slits in the bottom of the classification column. These particles are then sent to a condensation particle counter (CPC) where their concentrations are measured. By continuously varying (or scanning) the central rod voltage while measuring concentration, the entire particle size distribution can be determined to a high degree of accuracy (Knutson and Whitby, 1975).

In addition to obtaining size distribution scans, the SMPS can also be configured to continuously monitor particles in a single size range. This is accomplished simply by holding the central rod voltage constant. This instrument is capable of measuring size distributions for particles in the size range from $7 \mathrm{~nm}-0.5 \mu \mathrm{m}$, and can complete a doublescan size distribution measurement in as little as 60 seconds (Wang and Flagan, 1990). 
A schematic of test setup used in this study to measure plume characteristics and particulate matter emissions is illustrated in Figure 2.5. This testing of a heavy duty vehicle was conducted at the Old Dominion University (ODU) full scale wind tunnel facility in an effort to study and measure the exhaust plume (Boyce et al., 2000, Gautam et al., 2000). Programming was implemented and verified to facilitate truck loading in this study. Steady state tests were conducted with the vehicle cruising at a constant speed and a constant engine load. The truck was accelerated to a target speed (55 mph $(24.6$ $\mathrm{m} / \mathrm{s}$ )) by the driver. The power absorber was loaded incrementally until a target engine load was reached. The engine load was monitored by the truck's engine control unit provided by Cummins engine company. A cycle was also designed to simulate an onroad transient. The cycle consisted of 5 transient peaks, with each peak requesting the truck to acceleration from $40 \mathrm{mph}(17.88 \mathrm{~m} / \mathrm{s})$ to $55 \mathrm{mph}(24.6 \mathrm{~m} / \mathrm{s})$ during an eight seconds period. At the beginning of each peak, a maximum possible loading was applied to the power absorber. As soon as the truck reached $55 \mathrm{mph}(24.6 \mathrm{~m} / \mathrm{s})$, it was brought down to $40 \mathrm{mph}(17.88 \mathrm{~m} / \mathrm{s})$ using service brakes with a moderate power absorber loading. This procedure was repeated 5 times with about 40 seconds idle time between. The tunnel facility was equipped with a mobile test gantry that could be positioned remotely from a control at the facility. This mobile gantry was to map the dilution ratio at different locations in the plume and to measure the particle size distribution at different locations with Scanning Mobility Particle Sizer (SMPS) and collect total particulate matter samples on $70 \mathrm{~mm}$ glass fiber filter. Raw $\mathrm{CO}_{2}$ concentrations were measured by locating the probe in the exhaust stream and running raw exhaust stack to the analyzer cart. A three-inch $(76.2 \mathrm{~mm})$ diameter intake tube was located at the rear of the platform 
to provide samples from the dilute plume to laboratory trailer. A remote sampling system was attached to a traversing gantry. A large number of investigations have been published regarding the measurements of diesel particle size distribution, and the dilution processes on PM size distributions and concentrations. Particle size distribution was found to be dependent upon on the engine type, operating conditions, and the fuel used in test vehicles. An overview of summary of these investigations is presented in the next section.

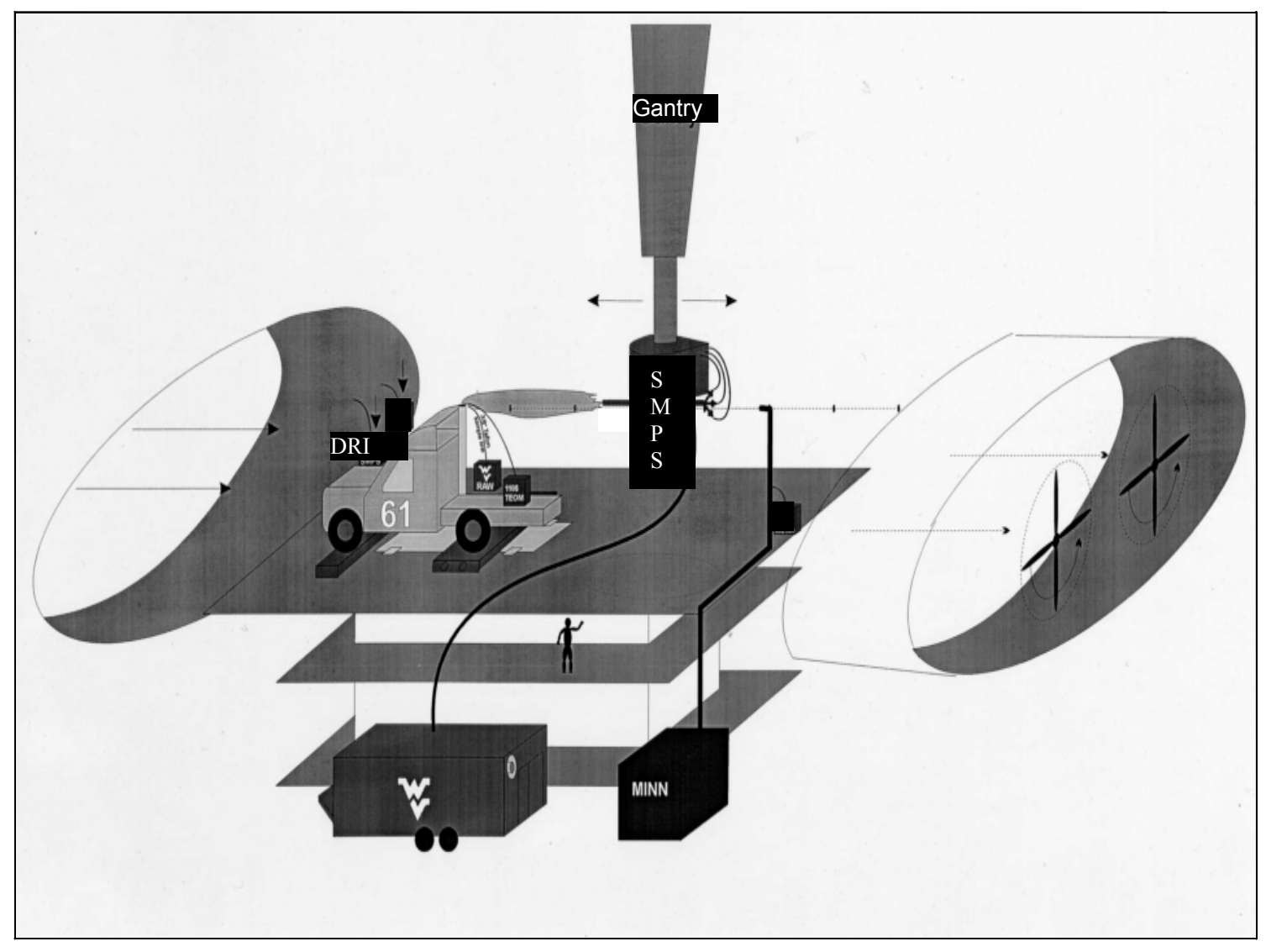

Figure 2.5 Schematic test setup in Langley wind tunnel (Gautam et al., 2000) 
Abdul-Khalek et al. (1998) made measurements of exhaust particle size distribution and number concentration of a medium-duty, turbo charged, after cooled, direct injection diesel engine. A compressed air ejector type mini-dilution system was used to rapidly dilute and cool the exhaust. The diluted exhaust was characterized by Scanning Mobility Particle Size, and Condensation Particle Counter (CPC). The engine which was equipped with an oxidation catalytic converter did not influence particle number concentration except in Mode 1 (2600 RPM, full load) where it increased number concentration by more than a factor of 10 due high exhaust temperature $\left(550^{\circ} \mathrm{C}\right)$. It was concluded that the dilution ratio seems to have a significant influence on the number concentrations and size distributions of particles. Increase in the primary dilution ratio from 4 to 60 results in significant decreases in number and volume concentration with number influence the most. However, it should be noted that the use of an ejector may have a profound effect on the particle size distribution. The sample experiences pressure of up to 25 " $\mathrm{Hg}(84.7 \mathrm{KPa})$ below atmospheric pressure. The ultra low pressures will lead to desorption of SOF from particles in the sample stream, followed most likely by nucleation in the cooled and diluted sample downstream of the ejector throat. To the best of this authors knowledge this phenomena has not been reported in literature.

Ahlvik et al. (1998) carried out experiments on a light-duty vehicle chassis dynamometer and on a heavy-duty engine. An electrical low pressure impactor (ELPI) and differential mobility analyzer (DMA) were used for the size distribution measurements both during steady state and driving cycle operations. Depending on the engine operation conditions, the size distributions were characterized by an accumulation 
mode peak in the range of 30 to $100 \mathrm{~nm}$ mobility diameter. Measurements during various transient and steady state driving cycles showed considerable differences in size distributions. It was concluded that the transient effects influence particle size distribution. However, the total number of particles was affected more by driving style and conditions.

Baumgard and Johnson (1996) investigated diesel PM size distributions and concentrations from a 1988 and a 1991 diesel engine using three different fuels and two exhaust control technologies, a ceramic particle trap (CPT), and oxidation catalytic converter (OCC). It was observed that the 1991 engine was designed for lower particulate matter mass emissions than the 1988 engine. For the 1991 engine, accumulation mode particles $(0.046-1.0 \mathrm{~mm}$ ) were lower by more than $80 \%$ (by volume) than the 1988 engine when low sulfur fuel $(0.03 \mathrm{wt} \%)$ was used. It was concluded that the trap was effective at removing the solid particles, but not as effective at removing the vapor phase material such as XOC (vapor phase HC collected XAD-II resin), SOF, and SO4. The OCC on the other hand was effective at oxidizing the $\mathrm{HC}, \mathrm{CO}, \mathrm{XOC}$ and SOF emissions due to the gases reacting with the precious metal catalytic surface, but had little effect on the solid particles that were present in the hot exhaust stream (Pataky et al., 1994).

Kruger et al. (1997) investigated the influence of exhaust gas after-treatment systems, and measurement conditions on particle size characteristics of exhaust from diesel engine. They observed that for a truck engine with a particle trap showed an increase in the number of particles less than $50 \mathrm{~nm}$, but a decrease in number of larger 
particles. Kruger et al. (1997) concluded that the trap drastically reduces the mass of particulate and the number of particles available as condensation adsorption sites. This enhances to the process of homogeneous nucleation of the exhaust gases and the creation of a large number of new particles.

Kittelson and Johnson (1991) discussed the impact of exhaust system temperature on particle measurement and provided recommendations to minimize the effects on diesel particulate matter sampling. Kittelson and Johnson (1991) calculated losses during the heavy-duty transient test for a typical test facility. Kittelson and Johnson's (1991) principal recommendations included the standardized engine and tunnel conditioning procedures, reduction of heat transfer during sampling and dilution, control of dilution ratio, tighter specification of the test cycle, and more accurate measurement of flows to and from the secondary dilution tunnel. Heat transfer during sampling and dilution cools the exhaust and helps in meeting maximum temperature requirements. However, it results in thermophoretic deposition of particles on sampling and dilution system surfaces. Eventually, these deposits are re-entrained in the exhaust stream. Re-entrainment is unpredictable and increases variability in mass measurements because of the increase in the number of coarse $(>1,000 \mathrm{~nm})$ particles. These particles are not necessarily representative of diesel aerosol and make aerosol size distribution measurement more difficult. Another problem related to temperature is the condensation of volatile matter on cool dilution tunnel walls. These volatiles are precursors of new nano-particles that form when the walls are heated, which leads of volatilization of condensed material. 
Ristovski et al. (2000) conducted tests to determine particle and gas emissions from two large compressed natural gas $(\mathrm{CNG})$ spark ignition (SI) engines. It was observed that a significant number of particles resized in the size range between 0.015 $\mu \mathrm{m}$ and $0.7 \mu \mathrm{m}$. This was measured using the Scanning Mobility Particle Sizer (SMPS). It was observed that there is a significant number of particles in the upper sub-micron range (with particles larger than $1 \mu \mathrm{m}$ ) for diesel emissions, while all the emissions for gas combustion are in the lower sub-micron (less than $50 \mathrm{~nm}$ ) range.

Graskow et al. (1998) measured the exhaust particulate emissions from a 4cylinder, 2.25 liter spark ignition engine. A single stage ejector diluter system was used to dilute and cool the exhaust sample for measurements. It was observed that spark ignition exhaust particle emissions were found to be highly unstable, unlike PM emissions from diesel engines.

Rickeard et al. (1996) investigated the impact of fuels (Swedish diesel, and gasoline) on the size distribution on PM emitted from gasoline and diesel light duty vehicles operated on a chassis dynamometer. Two instruments (quartz crystal micro balance - QCM and Scanning Mobility Particle Sizer - SMPS) were used to measure particle size from dilute exhaust in the constant volume sample (CVS) tunnel. Rickeard et al. (1996) used a log-normal distribution to fit the diesel particle count data and a peak was observed at about $110 \mathrm{~nm}$ for diesel vehicles, and $60 \mathrm{~nm}$ for gasoline vehicles. Noticeably, They observed a peak at $40 \mathrm{~nm}$ for some tests. It was inferred that this rare observation may be caused by the relatively low dilution ratios. This low dilution ratios is 
optimal for nuclei mode particle formation, but the residence time in the tunnel was not sufficient for coagulation and growth of the particles. The next section briefly discusses the theory of nucleation.

\subsection{NUCLEATION PROCESS}

Nucleation modes are brought about by the build-up of concentration of condensable vapor in the atmosphere, either generated in-situ by various chemical reactions, or brought in from distant sources by transport processes. The dependence of the particle yield on the production rate of condensable molecules is of particular interest, since it relates the likely increase in atmospheric aerosol concentrations to the increase in the emission of gas phase precursors, such as $\mathrm{SO}_{2}$ (Jones et al., 1994).

\subsubsection{Homogeneous Nucleation}

The initial formation of droplets from vapor is a complicated process. Droplets can be formed in the absence of condensation nuclei. Homogeneous nucleation or selfnucleation requires large saturation ratios $\left(S=p_{A} / p_{A}^{S}[T]\right)$, usually in the range of 2-10 to form nuclei particles. A detailed description of homogeneous nucleation is given by Nguyen et al. (1987). Nguyen et al. (1987) studied the formation of aerosol particles by homogeneous nucleation experimentally using a laminar flow aerosol generator. In their laboratory study, dry gas $\left(\mathrm{N}_{2}\right)$ saturated with dibutylphthalate (DBP) was cooled well below the saturation temperature causing the highly supersaturated vapor to nucleate. 


\subsubsection{Heterogeneous Nucleation}

The more common mode of particulate matter (PM) formation is nucleated condensation or heterogeneous nucleation. This process relies on existing sub-micrometer particles, called condensation nuclei, to serve as sites for condensation (Willeke and Baron, 1992). The sub-micrometer particles are usually ions, soluble or insoluble nuclei (Hinds, 1982). There is a possibility in diesel exhaust that there may be some ions and insoluble particles available to initiate heterogeneous nucleation, but homogeneous binary nucleation mode would be more likely to occur in the PM formation.

\subsubsection{Homogeneous Binary Nucleation in the $\mathrm{H}_{2} \mathrm{SO}_{4}-\mathrm{H}_{2} \mathrm{O}$ Mixture}

When two or more vapor species are present, neither of which is supersaturated, nucleation can still take place as along as the participating vapor species are supersaturated with respect to a liquid solution droplet. In the classical binary nucleation theory the rate of nucleation, $\mathrm{J}$, can be written in the form

$$
J=C \exp \left(-\Delta G^{*} / k T\right)
$$

where $\Delta \mathrm{G}^{*}$ is the free energy required to form a critical nucleus, $\mathrm{C}$ is the frequency factor, $\mathrm{k}$ is the Boltmann's constant, and $\mathrm{T}$ is the temperature. The binary homogenous nucleation was first proposed by Flood (1934), but it was not until 1950 that Reiss (1950) published a complete theory of binary nucleation. The most important binary nucleation system in the diesel exhaust is that of sulfuric acid and water. Doyle (1961) was the first

to publish predicted nucleation rates for the $\mathrm{H}_{2} \mathrm{SO}_{4}-\mathrm{H}_{2} \mathrm{O}$ system. His calculations showed that, even in air of relative humidity less than $100 \%$, extremely small amounts of $\mathrm{H}_{2} \mathrm{SO}_{4}$ vapor are able to induce nucleation. 
There are two versions of nucleation theory available in the literature, that is, Doyle (1961) and Wilemski (1984). Baumgard and Johnson (1996) used Doyle's version of the theory to predict $\mathrm{H}_{2} \mathrm{SO}_{4}-\mathrm{H}_{2} \mathrm{O}$ particle formation in diesel exhaust during dilution and did not consider sulfuric acid hydration. Further refinements were made by JaeckerVoirol and Mirabel (1989), and Kulmala et al. (1998) who examined the influence of hydrate formation in the $\mathrm{H}_{2} \mathrm{SO}_{4}-\mathrm{H}_{2} \mathrm{O}$ system. In this research, nucleation theory, including sulfuric acid hydration is applied.

The experimental results from Shi et al. (1999) suggested that sulfate $\left(\mathrm{SO}_{4}{ }^{2-}\right)$ was involved in the formation of new particles. The sulfate in diesel exhaust is derived from the fuel sulfur. Almost all the fuel sulfur is oxidized into $\mathrm{SO}_{2}$ during combustion, and several percent of $\mathrm{SO}_{2}$ is converted to $\mathrm{SO}_{3}$ at the lower temperatures in the exhaust pipe and dilution processes. When water vapor is present, the $\mathrm{SO}_{3}$ reacts with $\mathrm{H}_{2} \mathrm{O}$ and forms $\mathrm{H}_{2} \mathrm{SO}_{4}$ vapor. These $\mathrm{H}_{2} \mathrm{SO}_{4}$ and $\mathrm{H}_{2} \mathrm{O}$ vapors would be condensed in binary homogeneous nucleation.

Karcher (1996) reported the formation of $\mathrm{H}_{2} \mathrm{SO}_{4}-\mathrm{H} 2 \mathrm{O}$ agglomerates inside an exhaust plume of a jet engine. This suggested that emitted $\mathrm{SO}_{2}$ is oxidized by $\mathrm{OH}$ or $\mathrm{O}$ to $\mathrm{SO}_{3}$ that reacts with $\mathrm{H}_{2} \mathrm{O}$ to form gaseous $\mathrm{H}_{2} \mathrm{SO}_{4}$. The formation of $\mathrm{SO}_{3}$ is restricted only to the very young plume with less than $10 \mathrm{~ms}$ in age (Frenzel and Arnold, 1994). Frenzel and Arnold (1994) and Karcher (1996) investigated that gas-to-particle conversion and coagulation lead to the buildup of $\left(\mathrm{H}_{2} \mathrm{SO}_{4}\right)_{n}\left(\mathrm{H}_{2} \mathrm{O}\right)_{\mathrm{m}}$ clusters with an approximate size of 
$0.1 \mathrm{~nm}$ within a plume age of $0.5 \mathrm{~s}$ that will grow by $\mathrm{H}_{2} \mathrm{SO}_{4}$ condensation, coagulation, and water uptake to very small droplets making up an invisible contrail.

The physical events leading to heteromolecular nucleation and particle growth are depicted in Figure 2.6. For diesel exhaust, the binary vapor in Figure 2.6 (a) represents the $\mathrm{H}_{2} \mathrm{O}$ and $\mathrm{H}_{2} \mathrm{SO}_{4}$ vapor molecules. These molecules randomly collide with each other and form molecular clusters as shown in Figure 2.6 (b). In order for stable nuclei to form, a certain number of $\mathrm{H}_{2} \mathrm{O}$ and $\mathrm{H}_{2} \mathrm{SO}_{4}$ molecules must collide forming a molecular cluster that has sufficient energy to be stable in its environment (Figure $2.6 \mathrm{c}$ ). If this doesn't occur, the molecular clusters will eventually break up. Once the stable nuclei are formed, additional molecules will collide with the nuclei and the particles will grow as shown in Figure 2.6. (d). Whether or not particles are formed depends on the thermodynamics and kinetics of the system.

Meszaros and Meszaros (1989) investigated the atmospheric PM size distributions in both the winter and summer months. It was observed that more nuclei mode particles were found in summer than during the winter, and summer particle size distributions also indicated that $80 \%$ of the total particles consisted of $\mathrm{H}_{2} \mathrm{SO}_{4}$ and $20 \%$ were carbon in origin. Meszaros and Meszaros (1989) concluded that the $\mathrm{H}_{2} \mathrm{SO}_{4}$ particles were separate from the carbon particles, and are believed to form on their own.

When vapor starts to condense, energy must be conserved, causing the vapor free energy to decrease while the particle's surface free energy increases. If there is sufficient 
mixing energy such that molecular clusters form and overcome the surface free energy and if the clusters grow such that the free energy is greater than the minimum Gibbs energy, then clusters will be stable and continue to grow. If the free energy is below the minimum Gibbs energy, the clusters will evaporate.

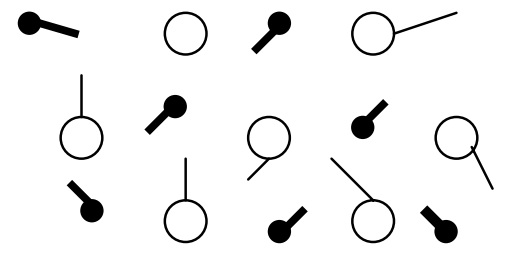

(a) Binary Vapor

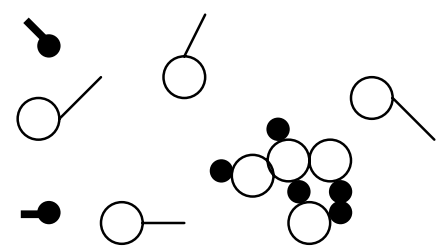

(c) Stable Nuclei

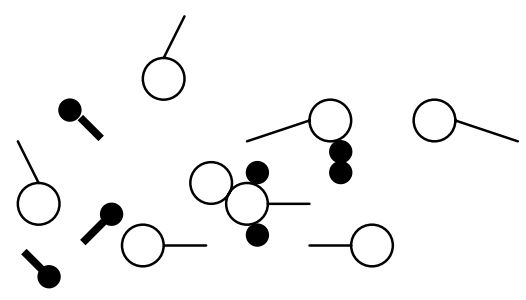

(b) Molecular Clusters

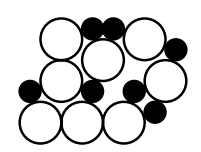

(d) Particle Growth

Figure 2.6. Events leading to binary homogeneous nucleation and particle growth (Baumgard and Johnson, 1996) 


\subsubsection{Effect of Saturation Ratio and Humidity on Nucleation}

The driving force for gas-to-particle conversion processes is the saturation ratio (S). For materials like the constituents of the soluble organic fraction (SOF) or sulfuric acid, the maximum saturation ratio is achieved during dilution and cooling of the exhaust (Kittelson, 1998). The relative rates of nucleation are an extremely nonlinear function of S. The old technology engines will take up supersaturated vapor quickly and prevent $\mathrm{S}$ from rising high enough to produce nucleation. On the other hand, in a modern low emission engine there is little surface area available to adsorb supersaturated vapors making nucleation more likely.

At saturation, $S=1$, nucleation of stable phase of $A$ from the gas phase does not occur. It is necessary that $\mathrm{S}>1$ for nucleation to occur. However, when two vapor species $\mathrm{A}$ and $\mathrm{B}$ are nucleating together, their individual saturation ratios need not exceed 1 for nucleation to occur. When the saturation ratio is raised above unity, there is an excess of monomer molecules over that at $\mathrm{S}=1$. These excess monomers bombard clusters and produce a greater number of clusters of larger size than exist at $S=1$. If the value of $S$ is sufficiently large, then sufficiently large clusters can be formed so that some clusters exceed a critical size, and then they can grow rapidly to form a new phase. Clusters that fluctuate to a size larger than the critical size will likely continue to grow to macroscopic size, whereas those smaller than the critical size most likely will shrink. The nucleation rate is the net number of clusters per unit time that grow past the critical size. 
Shi et al. (1998) reported that higher relative humidity and lower temperature lead to a higher nucleation rate, which is consistent with experimental findings. Temperature and relative humidity have been reported to be two key factors affecting the measured particle size distribution and concentration. Dilution ratio changes both temperature and gas phase $\mathrm{H}_{2} \mathrm{SO}_{4}$ partial pressure. In a real situation, the processes of nucleation, coagulation, and condensation will occur simultaneously. However, results of Shi et al. (1998) support, at least qualitatively, the hypothesis that $\mathrm{H}_{2} \mathrm{SO}_{4}-\mathrm{H}_{2} \mathrm{O}$ nucleation is a major cause of nanoparticle formation during dilution.

\subsubsection{Effect of Dilution Ratio on Diesel Particulate Matter}

Ahlvik et al. (1998) reported a higher number of ultrafine particles $(<0.1 \mu \mathrm{m})$ during operation of the tunnel at dilution ratios greater than 5. However, larger particles $(0.17 \mu \mathrm{m}-1 \mu \mathrm{m})$ increased in number at low dilution, but their number concentration remained several order of magnitudes below that of the smaller particles. The total number concentration was thus higher when higher dilution was applied. This tendency is related to coagulation and the possible nucleation phenomenon occurring in the dilution tunnel. In this study, both the chassis dynamometer and the transient test cell were equipped with full flow dilution tunnels. The constant flow was generated in both cases by using a critical flow venturi. In the engine test cell a secondary dilution tunnel is used

to further dilute the exhaust in order to achieve the desired temperature before the particulate filter. 
Abdul-Khalek et al. (1998) employed a the partial iso-kinetic mini-dilution system with primary and secondary dilution to study the effect of dilution ratio on the number and volume concentration of diesel particulate matter. Ejector pumps were used for primary and secondary dilution of exhaust, providing overall dilution ratio from 653000. For dilution ratio above about 60 , number as well as volume concentrations increased with decreasing dilution ratios. Nucleation was favored by the lower dilution ratios and more than likely increased the number concentration in the nuclei mode under those conditions.

Kittelson et al. (1998) reported three distinct stages of dilution in a cargo van - a rapid mixing stage (caused by the turbulent region between the tractor and trailer), followed by a steady dilution stage (similar to the flatbed's first stage), and by a second rapid mixing stage at the rear of the trailer. Data collected from the prototype dilution system, however, showed that it had not adequately simulated the ambient dilution that occurs in tractor-trailer exhaust. It is likely that allowing $500 \mathrm{msec}$ for the sample to undergo dilution from 7 to 70 allowed too much time for nucleation to occur. The plume data from this study on a cargo van indicated the ratio had already reached a dilution ratio of $\sim 250$ in 500 msec. Extrapolation of Kittelson's flatbed study (Kittelson et al., 1998) shows that the dilution ratio surpasses 50 (the top end of the "critical range" for nucleation) according to the authors in about $85 \mathrm{msec}$. Further characterization of the particles and dilution ratio in the plume, as it developed along the trailer and dispersed into the wake, was needed to define a performance envelope for any dilution sampling system. 
Bugarski (1999) reported that the dilution process had an important impact on the formation and transformation of the nano and ultrafine particles. Bugarski (1999) observed that lowering the dilution ratio resulted in diesel particulate matter distributions with slightly smaller count median diameters and higher or comparable peak concentrations. Bugarski (1999) also studied the effects of engine operating conditions, and observed that the emissions from the engine operated under transient conditions were characterized with significantly higher count median diameters and peak count concentrations than emissions from the engine operated at the steady-state conditions.

Baumgard and Johnson (1996) measured differences in particle size distributions in the engine exhaust, and in the dilution tunnel, and concluded that nuclei mode particles $(0.0075-0.046 \mu \mathrm{m})$ were formed in the dilution tunnel. Their experimental data indicated that the number of nuclei mode particles using the 1988 engine with federal on-highway diesel fuel with $0.32 \%$ (wt) sulfur was more than two orders of magnitude greater $\left(10^{9} \mathrm{vs}\right.$ $\left.10^{7}\right)$ than when using low sulfur fuel $(0.03 \% \mathrm{wt})$. They noticed that the percentage of particles in the nuclei mode was reduced from $98 \%$ to less than $33 \%$ with low sulfur fuel, which suggested that the majority of particles in nuclei mode originated from the fuel sulfur. These particles consisted of sulfate particle or sulfate particles provided the nuclei necessary for the nucleation process to occur. 


\subsubsection{Kelvin Effects on Nucleation}

The major aspect that characterizes the thermodynamics of atmospheric particles and droplets is their curved interface. The curvature of the surface modifies slightly the attractive forces between surface molecules, with the net result that the smaller the droplet the easier it is for molecules to leave the droplet surface. To prevent this evaporation, that is, to maintain mass equilibrium (no growth or evaporation), the partial pressure of vapor must be greater than $\mathrm{p}_{\mathrm{A}} \mathrm{S}$. For every droplet size there is one saturation ratio that will exactly maintain that size particle: too great a saturation ratio and the particle grows, too small and it evaporates. Conversely, for a given saturation ratio only those particle having a diameter $\mathrm{D}_{\mathrm{p}}{ }^{*}$ are stable, smaller ones evaporate, and larger ones grow.

The change of Gibbs energy, $\Delta \mathrm{G}$, accompanying the formation of a single droplet of pure material A of radius $R_{p}$ containing g molecules of the substance is as follows:

$$
\Delta G=-\frac{4}{3} \pi R_{p}^{3} \frac{k T}{v_{l}} \ln S+4 \pi R_{p}^{2} \sigma
$$

where $\mathrm{R}_{\mathrm{p}}$ is a radius of droplet, $\mathrm{k}$ is Boltzmann's constant, $\mathrm{T}$ is the temperature, $\mathrm{v}_{l}$ is the volume occupied by a molecule in the liquid phase, saturation ratio $S=p_{A} / p_{A}{ }^{S}$ and $\sigma$ is surface tension .

Figure 2.7. illustrates the behavior of $\Delta \mathrm{G}$ as a function of $\mathrm{R}_{\mathrm{p}}$. If $\mathrm{S}<1, \Delta \mathrm{G}$ increase monotonically with $\mathrm{R}_{\mathrm{p}}$. If $\mathrm{S}>1, \Delta \mathrm{G}$ consists of positive and negative contributions. At small values of $R_{p}$ the surface tension term dominates and the behavior of $\Delta G$ as a function of $R_{p}$ is close to that in the case of $S<1$. 


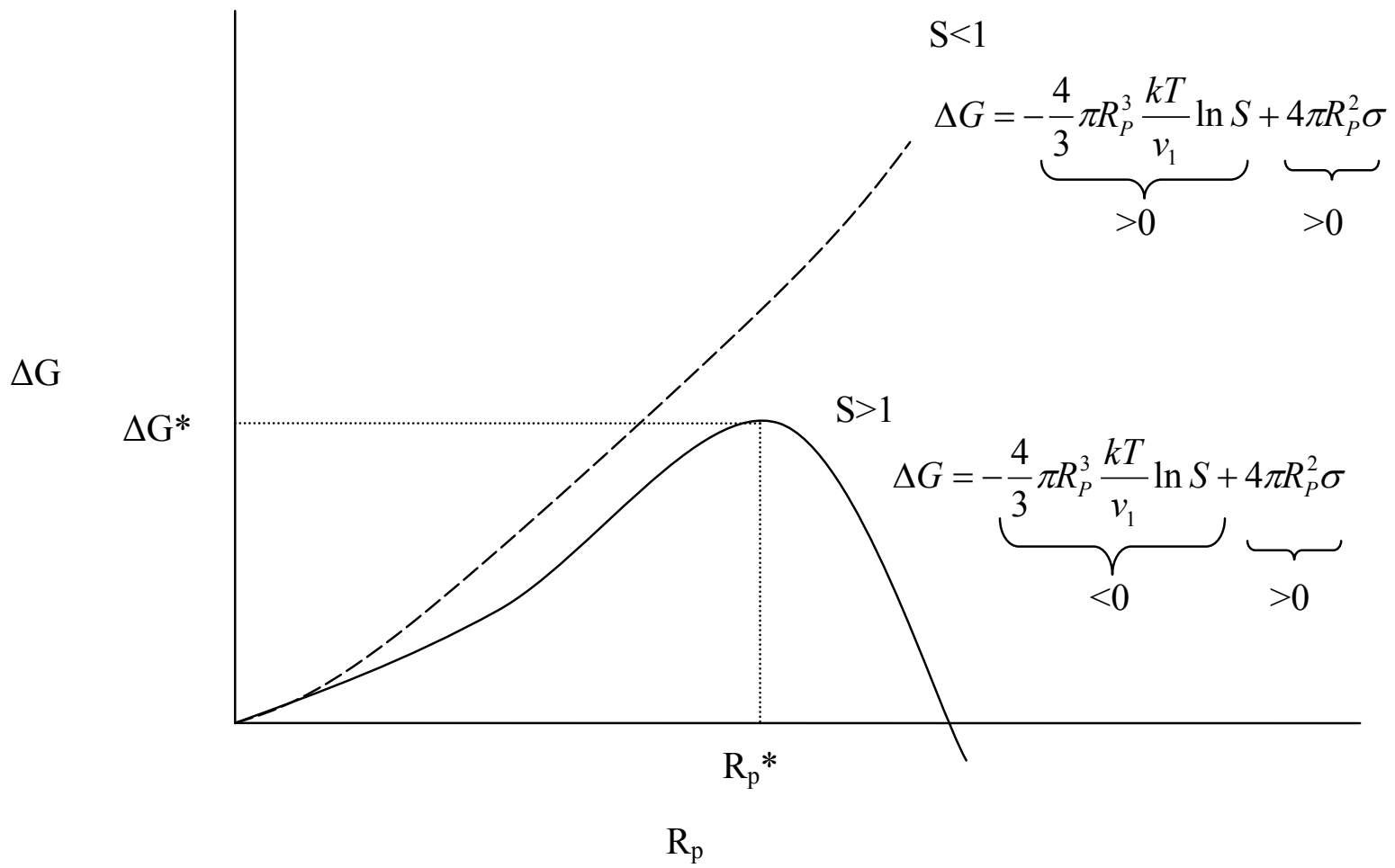

Figure 2.7. Gibbs free energy change for formation of a droplet radius Rp from a vapor with a saturation ratio S (Sienfeld and Pandis, 1997)

The change in Gibbs energy, $\Delta \mathrm{G}$, achieves a maximum at $\mathrm{R}_{\mathrm{p}}=\mathrm{R}_{\mathrm{p}}{ }^{*}$ which can be found by setting $\left(\partial \Delta G / \partial R_{P}\right)_{T, P}=0$,

$$
R_{P}^{*}=\frac{2 \sigma v_{l}}{k T \ln S}
$$

The corresponding value of the number of molecules at the critical size $g^{*}$ is

$$
g *=\frac{32 \pi \sigma^{2} v_{l}^{2}}{3(k T \ln S)^{3}}
$$

The value of $\Delta \mathrm{G}$ at $\mathrm{R}_{\mathrm{p}}=\mathrm{R}_{\mathrm{p}} *$ is 


$$
\Delta G^{*}=\frac{4}{3} \pi R_{P}^{* 2} \sigma=\frac{4}{3} \pi \sigma\left(\frac{2 \sigma v_{l}}{k T \ln S}\right)^{2}
$$

Since $\Delta \mathrm{G}$ is a maximum at $\mathrm{R}_{\mathrm{p}}=\mathrm{R}_{\mathrm{p}}{ }^{*}$, the equilibrium at that point is a meta-stable one, which means changing readily either to a more stable or less stable conditions.

Equation (2.4) can be rearranged so that equilibrium saturation ratio is given as a function of the radius of the droplet,

$$
\ln S=\frac{2 \sigma v_{l}}{k T R_{P}}=\frac{4 \sigma v_{l}}{k T D_{P}}
$$

Equation (2.4) is referred to as the Kelvin equation. The vapor pressure over a curved interface always exceeds that of the same substance over a flat surface as determined by using the Kelvin equation. However, the above equations are applicable to the equilibrium vapor pressure of a droplet of pure substance.

The vapor pressure of solvent over a solution consisting of a solute in a water with a flat surface is, by extension of Raoult'slaw, $p_{A s o l}^{0}=\gamma_{A} x_{A} p_{A}^{0}$, where $\mathrm{p}_{\mathrm{A}}^{0}$ is the vapor pressure of pure solvent water over a flat surface, $\gamma_{\mathrm{A}}$ is its activity coefficient in the solution, and $\mathrm{x}_{\mathrm{A}}$ is mole fraction of species A. Substituting this expression into the Kelvin equation:

$$
\ln \frac{p_{A}}{p_{A}^{0}}=\frac{A}{D_{p}}-\frac{B}{D_{p}^{3}}
$$

where $A=4 \sigma \bar{v}_{w} / R T$ and $B=6 n_{B} \bar{v}_{w} / \pi$. 
For a pure water drop the vapor pressure over a curved interface always exceeds that over a flat interface. By contrast, the vapor pressure $\mathrm{p}_{\mathrm{A}}$ over an aqueous solution droplet may be larger or smaller than the vapor pressure of pure water over a flat surface $\mathrm{p}_{\mathrm{A}}{ }^{0}$ depending upon the magnitude of the solute-effect term, $\mathrm{B} / \mathrm{D}_{\mathrm{p}}{ }^{3}$, relative to the curvature term, $\mathrm{A} / \mathrm{D}_{\mathrm{p}}$.

Figure 2.8. shows a sketch of $\ln \mathrm{p}_{\mathrm{A}} / \mathrm{p}_{\mathrm{A}}{ }^{0}$ as a function of $\mathrm{D}_{\mathrm{p}}$. The value of $\mathrm{D}_{\mathrm{p}}$ at which $\mathrm{p}_{\mathrm{A}}=\mathrm{p}_{\mathrm{A}}{ }^{0}$ is denoted $\mathrm{D}_{\mathrm{pp}}$, called the potential diameter, and is given by $(\mathrm{B} / \mathrm{A})^{1 / 2}$ where $A=4 \sigma \bar{v}_{w} / R T$ and $B=6 n_{B} \bar{v}_{w} / \pi$. The curve of $\ln \mathrm{p}_{\mathrm{A}} / \mathrm{p}_{\mathrm{A}}{ }^{0}$ reaches a maximum at $\mathrm{D}_{\mathrm{pc}}$, the critical diameter, which is equal to $(3 \mathrm{~B} / \mathrm{A})^{1 / 2}$. The steeply rising portion of the Köhler curve (Figure 2.8) represents a region where solute effects dominate, and the $-\mathrm{B} / \mathrm{D}_{\mathrm{p}}{ }^{3}$ term leads to a rapid increase in $\mathrm{p}_{\mathrm{A}} / \mathrm{p}_{\mathrm{A}}{ }^{0}$ to unity as $\mathrm{D}_{\mathrm{p}}$ increases. When $\mathrm{p}_{\mathrm{A}}=\mathrm{p}_{\mathrm{A}}{ }^{0}$, both terms are equal. Beyond $\mathrm{D}_{\mathrm{pp}}$, $\mathrm{S}$ becomes greater than 1 and the Kelvin effect begins to predominate. At the point of maximum, or critical saturation, the Kelvin effect contribution is three times as large as the solute effect term.

\subsection{COAGULATION}

Diesel exhaust is a very complex system where several particle transformation processes, for example, nucleation, coagulation, and condensation among others may occur simultaneously. Condensation can be viewed as an extension of nucleation and adsorption. In case of adsorption, as saturation ratios approach and exceed saturation, continued addition of the organics to the particles comes by condensation. Nucleation 


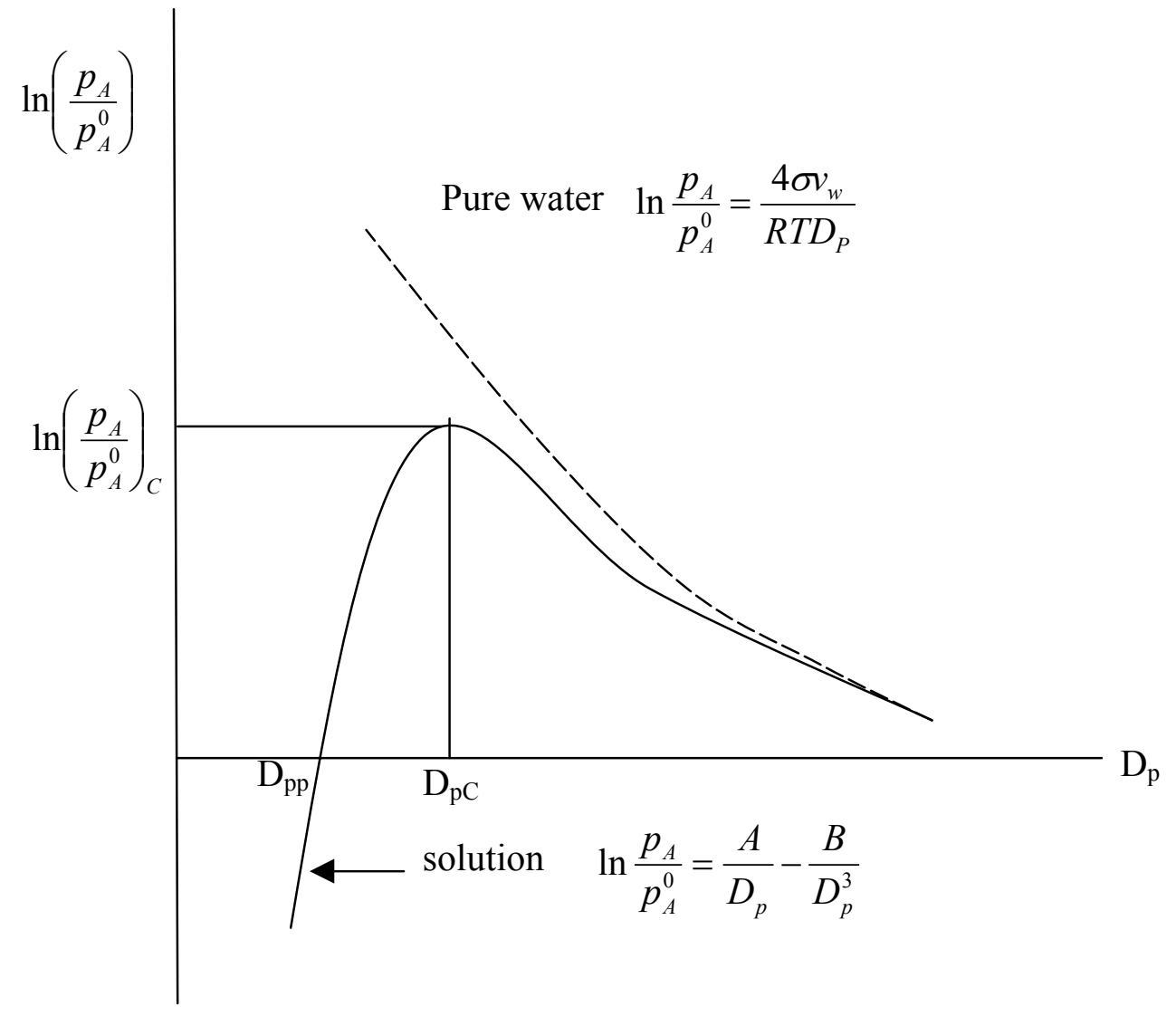

Figure 2.8. The ratio of the vapor pressure $\mathrm{p}_{\mathrm{A}}$ of a solvent $\mathrm{A}$ over a solution droplet to its pure component vapor pressure over a flat surface $\mathrm{p}_{\mathrm{A}}{ }^{0}$, as a function of the diameter of the droplet (Curves of this type are called Köhler curves). (Sienfeld and Pandis, 1997)

contributes to the initial formation of a droplet or particle, and coagulation attributes to increase in particle size. Up to this point, the physics and chemistry of particles have been considered from the point of view of behavior of a single particle. This section is focused on a population of particles that interact with each other. 
The theory of coagulation deals with the process of adhesion or coalescence of particles when they come in contact with one another. This theory describes the particle size distribution as a function of time and space as it undergoes changes due to various external factors, for example relative humidity, dilution and ambient temperature. Particles suspended in a fluid (or air) may come into contact, because of Brownian motion or as a result of motion produced by hydrodynamic, electrical, gravitational, or other forces. Brownian coagulation is often referred to as thermal coagulation.

The evolution of the particle size distribution due to coagulation is represented by the following integro-differential equation (Muller, 1928):

$$
\begin{aligned}
& \frac{\partial n(v, t)}{\partial t}=\frac{1}{2} \int_{v^{*}}^{v} \beta(v-\widetilde{v}, \widetilde{v}) n(\widetilde{v}, t) n(v-\widetilde{v}, t) d \widetilde{v}-\int_{0}^{\infty} \beta(v, \widetilde{v}) n(v, t) n(\widetilde{v}, t) d \widetilde{v}, \\
& n(v, 0)=n_{0}(v), \\
& n(v, t)=0, v<v^{*}
\end{aligned}
$$

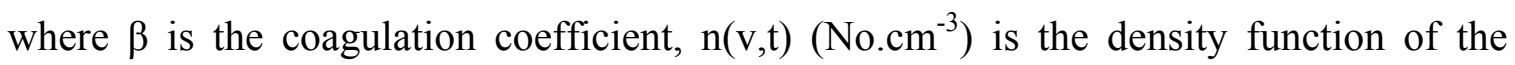
particle number size distribution, $\mathrm{v}$ is particle volume, $\mathrm{t}$ is the time, $\mathrm{v}^{*}$ is the minimum particle volume, $\bar{v}$ is the dummy integral variable of Equation (2.9), and $\mathrm{n}(\mathrm{v}, \mathrm{t}) \mathrm{dv}$ is the number of particles per unit volume of air, with volumes between $\mathrm{v}$ and $\mathrm{v}+\mathrm{dv}$. Difficulties in solving this equation arise in the evaluation of the integrals on the righthand side.

The simple monodisperse coagulation assumes that all particles are monodisperse, and will stick if they contact one another, and then grow slowly. But, particles exhibit Brownian motion and diffusion like gas molecules, and these phenomena occur at a much 
slower pace. Consequently, the diffusion coefficients for particles are about a million times smaller than those for gas molecules (Willeke and Baron, 1992).

The change in number concentration over a period of time $t$ may be written as :

$$
N(t)=\frac{N_{0}}{1+N_{0} k t}
$$

where $\mathrm{N}(\mathrm{t})$ is the number concentration at time $t, \mathrm{~N}_{0}$ is the initial number concentration and $\mathrm{k}$ is the coagulation coefficient. As number concentration decreases, particle size increases, but for a contained system with no losses particle mass will remain constant. Particle size is proportional to the cube root of particle volume, and, consequently, it is also proportional to the inverse cube root of number concentration:

$$
d(t)=d_{0}\left(\frac{N_{0}}{N(t)}\right)^{1 / 3} \quad \text { or } \quad d(t)=d_{0}\left(1+N_{0} K t\right)^{1 / 3}
$$

Above equations are correct for liquid droplets and approximately correct for solid particles that form compact clusters (Hinds, 1982 ).

In a "real-world" case, a poly-disperse collection of particles rather than monodisperse are encountered, and the situation is more complicated. Because the coagulation process is governed by the rate of diffusion of particles to the surface of each particle, the process is enhanced when small particles with their high diffusion coefficients diffuse to a large particle with its large surface. A tenfold difference in particle size produces a three-fold increase in coagulation, and a 100-fold difference results in a more than 25 fold increase in coagulation rate. The use of Equation (2.11) for polydisperse particles requires the use of numerical methods because coagulation for 
every combination of particle sizes has a different value of $\mathrm{k}$ and has to be calculated separately (Zebel, 1966).

\subsubsection{TECHNIQUES FOR SOLVING COAGULATION GOVERNING EQUATIONS}

The study of particle system behavior is relevant to a variety of fields such as air pollution, combustion and chemical engineering science. To understand the behavior of particle systems, it is necessary to be able to simulate by means of mathematical models the dynamic behavior of the particle population. Such simulations allow us to reconcile the theoretical description of the physical principles that govern particle systems with the experimental observation of these systems. Discrepancies between theory and experiment can then be analyzed and utilized to suggest theoretical advances and further experiments in the study of particle dynamics. To that end, several mathematical models have been developed to simulate the physical processes that affect particles. Such processes include coagulation, growth due to condensation of gases, shrinkage due to evaporation, nucleation of new particles, sedimentation, and deposition on surfaces.

\subsubsection{1 “J-space" Transformation}

Suck and Brock (1979), Tsang and Brock (1982, 1983), and Yom and Brock (1984) have developed mathematical models to simulate particle number distribution, $\mathrm{n}(\mathrm{v}, \mathrm{t})$, when it is affected by either coagulation or condensation/evaporation. Suck and Brock (1979) performed the following change of variable, the so-called J-space transformation:

$$
V(J)=v\left(J_{0}\right) \exp \left[\alpha\left(J-J_{0}\right)\right]
$$


Where $J$ and $J_{0}$ are integers such that $J \geq J_{0}, J_{0}>0$, and $\alpha$ is an adjustable parameter.

The particle distribution function in J-space is then represented by the following function:

$$
\psi(J, t)=\alpha v(J) n(v(J), t)
$$

The coagulation equation may then be rewritten for the J-space distribution function as follows:

$\frac{\partial \psi(J, t)}{\partial t}=\int_{J_{0}}^{J_{h}} \beta_{J}\left(\widetilde{J}, J^{\prime}\right) \frac{v(J)}{v(\widetilde{J})} \psi(\widetilde{J}, t) \psi\left(J^{\prime}, t\right) d J^{\prime}-\int_{J_{0}}^{\infty} \beta_{J}\left(J, J^{\prime}\right) \psi(J, t) \psi\left(J^{\prime}, t\right) d J^{\prime}$

where

$$
\begin{aligned}
& \widetilde{J}=J+\frac{1}{\alpha} \ln \left\{1-\exp \left[\alpha\left(J-J^{\prime}\right)\right]\right\} \\
& J_{h}=J-\frac{\ln 2}{\alpha} \\
& \beta_{J}\left(\widetilde{J}, J^{\prime}\right)=\beta\left(v-v^{\prime}, v^{\prime}\right)
\end{aligned}
$$

The integrals in Equation (2.14) are then represented by means of cubic spline functions, and a subroutine based on the method of Gear (1971) is used to integrate the resulting ordinary differential equations with respect to time. The solution of J-space transformation could be rewritten by following forms of size distribution functions in terms of the J-space distribution functions:

$$
\begin{aligned}
& \frac{d N}{d(\ln D)}=\{3 / \alpha\} \Psi(J) \\
& \frac{d M}{d(\ln D)}=(3 / \alpha) m(J) \Psi(J) \\
& \text { and } \\
& \frac{d S}{d(\ln D)}=\left(\frac{12 \pi}{\alpha}\right)\left(\frac{3}{4 \pi}\right)^{2 / 3}\{m(J) / \rho\}^{2 / 3} \Psi(J)
\end{aligned}
$$


The models based on a continuous size distribution provide an accurate solution for both coagulation and condensation.

\subsubsection{Asymptotic Solution}

The asymptotic technique called "the saddlepoint method" is applied to the inverse Laplace transform of the particle spectrum. Pilinis and Seinfeld (1987) presented the asymptotic solution for Equation (2.9). Using dimensionless parameters, Equation (2.9) could be written as follows:

$$
\begin{aligned}
& \frac{\partial \bar{n}(K n, \tau)}{\partial \tau}= \xi\left\{\frac{1}{2} \int_{K n_{b}}^{K n} \beta\left[K n^{\prime},\left(K n^{-3}-K n^{\prime-3}\right)^{-1 / 3}\right]\right. \\
& \times \bar{n}\left(K n^{\prime}, \tau\right) \bar{n}\left[\left(K n^{-3}-K n^{\prime-3}\right)^{-1 / 3}, \tau\right]\left[\frac{\left(K n^{-3}-K n^{\prime-3}\right)^{-1 / 3}}{K n}\right]^{4} d K n^{\prime} \\
& \\
&\left.-\bar{n}(K n, \tau) \int_{K n_{b}}^{K n_{a}} \beta\left(K n, K n^{\prime}\right) \bar{n}\left(K n^{\prime}, \tau\right) d K n^{\prime}\right\}
\end{aligned}
$$

with an initial condition

$$
\bar{n}(K n, 0)=\bar{f}(K n)
$$

where

$$
\begin{aligned}
& \bar{n}(K n, \tau)=-\frac{\lambda_{n}(D, t)}{N_{0} K n^{2}}, \quad \bar{S}_{0}(K n, \tau)=\frac{S_{0}(D, t) \lambda^{3}}{D N_{0} K n^{2}}, \quad \alpha(K n, \tau)=\frac{\alpha(D, t) \lambda^{2}}{D}, \\
& \xi=\frac{N_{0} k T \lambda^{2}}{D \eta}, \quad \bar{I}(K n, \tau)=\phi I(D, t), \quad \tau=\frac{D t}{\lambda^{2}}
\end{aligned}
$$

and $\mathrm{Kn}$ is the Knudsen number, $2 \lambda / \mathrm{D}$; $\mathrm{T}$ is the absolute temperature; $\varnothing$ is a growth coefficient depending on the growth mechanisms, $\varnothing=\mathrm{N}_{1} \mathrm{v}_{1} \mathrm{D} / \lambda$, D is the diffusivity of the vapor molecules, $\lambda$ is the mean free path, $\sigma(\tau)$ is the coefficient accounting for any temporal variation of the growth rate; $\mathrm{N}_{1}, \mathrm{v}_{1}$ are the gas phase concentration and the 
volume of the condensing molecules, respectively, $\mathrm{N}_{0}$ is the initial total particle number concentration, $\mathrm{t}$ is time, $\tau$ is dimensionless time and $\eta$ is the viscosity. The Equation (2.16) is multiplied by the parameter $\xi$. This parameter depends on conditions (growth or deposition) in the system and the total particle concentration. If $\xi$ is small, then the coagulation contribution to the evolution of $\mathrm{n}(\mathrm{Kn}, \tau)$ is correspondingly small. The dimensionless total particle number concentration, $\bar{N}(\tau)=\mathrm{N}(\tau) / \mathrm{N}_{0}$, is given by

$$
\bar{N}(\tau)=\int_{K n_{b}}^{K n_{a}} \bar{n}(K n, \tau) d K n
$$

In general, the integral in Equation (2.17) must be evaluated numerically. In the special case of $\beta(\mathrm{Kn}$ ', Kn' $)=2 \mathrm{C}$ the integral can analytically be evaluated to give

$$
\bar{N}(\tau)=1-\xi C \tau+O\left(\xi^{2}\right)
$$

where $\mathrm{Kn}^{\prime}$ is the dummy integral variable of Equation (2.16), and $\mathrm{Kn}^{\prime \prime}$ is $\left(\mathrm{Kn}^{-3}-\mathrm{Kn}^{1-3}\right)^{-1 / 3}$, and $\xi$ is the variable of integration which depends on the total particle concentration. The exact result for the total number in the case of a constant coagulation coefficient is

$$
\bar{N}(\tau)=\frac{1}{1+\xi C \tau}
$$

Equation (2.18) contributes the first two terms in the Taylor series expansion of Equation (2.19).

\subsubsection{Discrete Method}

Tambour and Seinfeld (1980) assumed that each particle consisted of an integer multiple of elementary units (or monomers), which were taken to be molecules. Assuming that the agglomeration process conserves monomers, the conservation Equation for each $\mathrm{k}$-mer is 


$$
\frac{d n_{k}}{d t}=\frac{1}{2} \sum_{i+j=k} \beta_{i j} n_{i} n_{j}-n_{k} \sum_{i=1}^{\infty} \beta_{i k} n_{i}
$$

where $n_{k}(t)$ is the number of particles per unit volume of the fluid containing $k$ units. The coefficient $\beta_{\mathrm{ij}}$ is the collision frequency or the agglomeration coefficient for particles containing $\mathrm{i}$ and $\mathrm{j}$ monomers. Now, for any arbitrary initial distribution, Equation (2.20) can be written in the following form:

$$
n_{k}(t)=\frac{N_{\infty}(0) \sum_{m=0}^{\infty} A_{m k}\left(\frac{t}{\tau}\right)^{m}}{\left(1+\frac{t}{\tau}\right)^{K+1}}
$$

where $\tau$ is the dimensionless time, $t$ is the time, $\mathrm{N}_{\infty}$ is the total particle number concentration (sum of all $\mathrm{k}$ particles), and $\mathrm{A}_{\mathrm{mk}}$ is the collection efficiency between m-and $\mathrm{k}$ - particle. As in the solution for $\mathrm{N}_{\infty}(\mathrm{t})$, the moments, $\mathrm{x}_{\mathrm{q}}$ and $\mathrm{y}_{\mathrm{q}}$, will be taken as constants, and will then be reevaluated as a function of time.

Discrete (sectional) approaches simulate coagulation very well but require a fine size resolution to minimize numerical diffusion in the simulation of condensation (Seigneur et al., 1986).

\subsubsection{Moment Method}

For this method the evolving spectrum is expressed in a series of orthogonal functions with coefficients that depend upon the power moments of the spectrum. If the series is substituted into the kinetic equation for the spectrum, the result is an infinite system of equations describing the evolving power moments. The orthogonality property allows the infinite series to be truncated without producing a "closure problem" in the set 
of equations for the moments. This truncated series is, an approximate solution for the evolving spectrum (Golovin, 1963; Enukashvili, 1964). Peterson et al. (1978) used this method of approach through the Laplace transformation with respect to volume.

The equation defining the time evolution of the total number of particles per unit volume of fluid,

$$
M_{0}(t)=\int_{0}^{\infty} n(v, t) d v
$$

and the total volume of particles per unit volume of fluid,

$$
M_{1}(t)=\int_{0}^{\infty} v n(v, t) d v
$$

is obtained from Equation (2.22) by integrating over all volumes, or by multiplying by $\mathrm{v}$ and then integrating over all volumes. Much useful information regarding the time evolution of the particle is obtained by analyzing these moment solutions.

\subsubsection{Parametrized Representation}

Whitby (1981) method is based on the parametrization of the coagulation and condensation processes and describes the evolution of the number and volume concentrations. The fixed number of parameters involved in the model formulation does not allow for variation of the resolution of the particle size distribution, and the parametrized approach is constrained by this definition. This distribution is represented by two lognormal distribution as follows:

$$
\begin{aligned}
n(d, t)= & \frac{N_{n}(t)}{(2 \pi)^{1 / 2} \log \left[\sigma_{n}(t)\right]} \exp \left[-\frac{1}{2}\left(\frac{\log \left[d / d_{N n}(t)\right]}{\log \sigma_{n}(t)}\right)^{2}\right] \\
& +\frac{N_{a}(t)}{(2 \pi)^{1 / 2} \log \left[\sigma_{n}(t)\right]} \exp \left[-\frac{1}{2}\left(\frac{\log \left[d / d_{N a}(t)\right]}{\log \sigma_{a}(t)}\right)^{2}\right] .
\end{aligned}
$$


where the subscripts $\mathrm{n}$ and a refer to the nuclei and accumulation modes, respectively, $\mathrm{N}_{\mathrm{n}}$ and $\mathrm{N}_{\mathrm{a}}$ represent the total particle number concentrations in each mode, $\mathrm{d}_{\mathrm{Nn}}$ and $\mathrm{d}_{\mathrm{Na}}$ are the geometric mean particle diameters, and $\sigma_{\mathrm{n}}$ and $\sigma_{\mathrm{a}}$ are the standard deviations. Similarly, the bimodal volume distribution, $\mathrm{V}(\mathrm{d}, \mathrm{t})$ represents the particle volume concentrations as a function of $\log (\mathrm{d})$. It is represented by the sum of two log-normal distributions as follows:

$$
\begin{aligned}
V(d, t)= & \frac{V_{n}(t)}{(2 \pi)^{1 / 2} \log \left[\sigma_{n}(t)\right]} \exp \left[-\frac{1}{2}\left(\frac{\log \left[d / d_{V n}(t)\right]}{\log \left[\sigma_{n}(t)\right.}\right)^{2}\right] \\
& +\frac{V_{a}(t)}{(2 \pi)^{1 / 2} \log \left[\sigma_{a}(t)\right]} \exp \left[-\frac{1}{2}\left(\frac{\log \left[d / d_{V a}(t)\right]}{\log \left[\sigma_{a}(t)\right.}\right)^{2}\right]
\end{aligned}
$$

where $V_{n}$ and $V_{a}$ represent the total particle volumes in each mode, and, $d_{V n}$ and $d_{V a}$ are the geometric mean particle diameters. A third log-normal distributions with constant parameters was added in this study to represent the coarse mode.

In the original formulation (Whitby, 1981), the coagulation equations consist of a set of four ordinary differential equations representing the temporal evolution of these four parameters, and four algebrasic equations calculating the mean diameters; the standard deviation were kept constant:

$$
\begin{aligned}
& \frac{d N_{n}(t)}{d t}=-K_{n n} N_{n}^{2}(t)-K_{n a} N_{n}(t) N_{a}(t), \\
& \frac{d N_{a}(t)}{d t}=K_{n a} N_{n}(t) N_{a}(t)-K_{a a} N_{a}^{2}(t), \\
& \frac{d V_{n}(t)}{d t}=-K_{n a} N_{a}(t) N_{n}(t) \frac{\pi}{6}\left(d_{N n}\right)^{3} \exp \left(4.5 \ln ^{2} \sigma_{n}\right), \\
& \frac{d V_{a}(t)}{d t}=K_{n a} N_{a}(t) N_{n}(t) \frac{\pi}{6}\left(d_{N n}\right)^{3} \exp \left(4.5 \ln ^{2} \sigma_{n}\right)
\end{aligned}
$$


The parameters $\mathrm{K}_{\mathrm{nn}}, \mathrm{K}_{\mathrm{aa}}$, and $\mathrm{K}_{\mathrm{na}}$ are the nuclei intramodal (inside nuclei or accumulation mode), accumulation intramodal, and nuclei/accumulation intermodal (between the nuclei and accumulation mode) coagulation coefficients, respectively. These parameters depend on the standard deviations and mean diameters of the modal distribution (Whitby, 1985). The first equation represents the decrease in particle numbers in the nuclei mode due to intramodal coagulation of nuclei mode particles and intermodal coagulation of nuclei mode particles with accumulation model particles. The second equation represents the change in particle number in the accumulation mode due to intermodal coagulation of nuclei mode particle with accumulation mode particles and intramodal coagulation of accumulation mode particles. The change in volume concentrations results from intermodal coagulation only. This value is calculated from the intermodal coagulation term of the number concentrations and converted into volume concentrations.

The parametrized model based on log-normal modal size distributions is computationally efficient but tends to overestimate the rate of coagulation and the peak particle concentration resulting from condensational growth (Seigneur et al., 1986).

\subsubsection{Similarity Solution}

A method of solving certain coagulation problems has been developed based on the use of a similarity transformation for the size distribution function. The similarity transformation for the particle size distribution is based on the assumption that the 
fraction of the particles in a given size range is a function only of particle volume normalized by the average particle volume (Friedlander and Wang,1966):

$$
\frac{n d v}{N_{\infty}}=\psi\left(\frac{v}{\bar{v}}\right) d\left(\frac{v}{\bar{v}}\right)
$$

where $\bar{v}=V / N_{\infty}$ is the average particle volume ( $\mathrm{V}$ is the total particle volume, and $N_{\infty}$ is the total number concentration), $\mathrm{n}$ is the number concentration of particles, $\psi\left(\frac{v}{\bar{v}}\right)$ is the self-preserving spectrum, $\mathrm{N}_{\infty}$ is the total particle number concentration, and $\mathrm{v}$ is the particle volume. Both sides of Equation (2.27) are dimensionless. Rearranging the result yields

$$
n(v, t)=\frac{N_{\infty}^{2}}{V} \psi(\zeta)
$$

where $\zeta=v / \bar{v}=N_{\infty} v / V$. There are also the integral relations:

$$
N_{\infty}=\int_{0}^{\infty} n d v
$$

and

$$
V=\int_{0}^{\infty} n v d v
$$

In terms of the distribution function $n_{a}(a)$, the similarity transformation takes the form

$$
n_{a}(a, t)=\frac{N_{\infty}^{4 / 3}}{V^{1 / 3}} \psi_{a}\left(\zeta_{a}\right)
$$

where $\zeta_{a}=a\left(N_{\infty} / V\right)^{1 / 3}$. Both $\mathrm{N}_{\infty}$ and $\mathrm{V}$ are functions of time. In the simplest case, no material is added or lost from the system, and V is constant. The number concentration $\mathrm{N}_{\infty}$ decreases as coagulation takes place. If the size distribution corresponding to any value of $\mathrm{N}_{\infty}$ and $\mathrm{V}$ are known, the distribution for any value of $\mathrm{N}_{\infty}$, corresponding to a 
different time, can be determined from Equation (2.28) if $\psi(\zeta)$ is known. The shapes of the distribution at different times are similar when reduced by a scale factor. For this reason, the distribution is said to be "self-preserving."

\subsubsection{Direct Simulation by Monte Carlo Method}

Brown (1956) defined a Monte Carlo method as any procedure which involves the use of statistical sampling techniques to approximate the solution of a mathematical or physical problem. Such procedures have been used to "directly simulate" the growth process of particles and cloud droplets.

Husar (1970) considered a population of particles coagulating in a random motion field. He found that Monte Carlo methods were easy to apply and did not use excessive amounts of computer time. Since individual trajectories and collisions can be followed in this type of simulation, particles resulting from the coagulation of initially spherical particles can be of various shapes. In fact Husar (1970) has produced particle shapes which resembled those observed for combustion generated particles.

\subsubsection{OTHER COAGULATION MODELS IN THE LITERATURE}

Over the past 15 years, several aerosol models have been developed relying on thermodynamic equilibrium principles to predict the composition and physical state of inorganic atmospheric aerosols. It has been widely assumed that volatile species in the gas and aerosol phases are in chemical equilibrium (Stelson and Seinfeld, 1982a, b; Bassett and Seinfeld, 1983; Saxena, et al., 1986; Pilinis and Seinfeld, 1987). Previous 
work by Hildemann et al. (1984) has confirmed the general agreement of ambient inorganic aerosol measurements with those predicted by thermodynamic equilibrium. However, in cases where the equilibrium timescale is long relative to the residence time of particles in a given environment, thermodynamic eqilibrium may not be a good approximation (Wexler and Seinfeld, 1990; Meng and Seinfeld, 1996). Aerosol models based on thermodynamic equilibrium include EQUIL (Bassett and Seinfeld, 1983), MARS (Saxena et al., 1986), SEQUILIB (Pilinis and Seinfeld, 1987), AIM (Wexler and Seinfeld, 1991), SCAPE (Kim et al., 1993a), and SCAPE2 (Meng et al., 1995). These models have been developed for atmospheric simulations and thus, computational efficiency was a major objective during their development. To attain computational efficiency, assumptions were made to simplify the problem at hand and allow use of the equilibrium models in Eulerian atmospheric chemical transport models. Threedimensional models capable of tracking aerosol dynamics (transport of gases to particles, particle formation, and size distribution) have been developed in SEQUILIB (Pilinis and Seinfeld, 1988; Lurmann et al., 1997) to simulate the behavior of organic and inorganic aerosols. Although limited comparisons among equilibrium models have been conducted (Waxler and Seinfeld, 1991; Kim et al., 1993b), no detailed comparison to observations of aerosol behavior has been performed in part due to limited availability of such measurements; the ability to reproduce laboratory aerosol observations is yet unknown. The equilibrium models mentioned may agree with each other within a $5-10 \%$ error, but if they cannot reproduce observed aerosol behavior, their results may introduce errors in atmospheric simulations. 
During the dilution process, the formation and growth of aerosol particles by condensation is the principal method of aerosol production in nature. It is the most important mass transfer process between the gas phase and the particle phase., which is described in next section.

\subsection{CONDENSATION}

Condensation is similar to nucleation in that it occurs when saturation ratio is greater than unity, except that the material condenses onto the surface of particles instead of forming new particles (Hinds, 1982). Ahlvik et al. (1998) reported that both condensation and nucleation are dependent upon dilution ratio. It was found that lower dilution ratios lead to an increase in the saturation pressure itself, because of the respective increase in the dilution temperature. Condensation affects both the mass and the number concentration by forming liquid ultra-fine particles in the dilution tunnel.

The opposite of the condensation growth process is the closely related process of evaporation. This process is important for spray-drying applications and is involved in the production of nuclei, such as sea salt nuclei, which are originally formed as droplets and evaporate to form nuclei that serve as sites for subsequent condensation. 


\section{$2.8 \quad$ SUMMARY}

The previous sections have shown that the primary carbon spherules are formed during the combustion process. The majority of these particles are quickly oxidized in the combustion chamber, but the fraction that survives agglomerates and forms the typical long chain or cluster particles associated with diesel exhaust (Vuk et al., 1976). During the dilution process, the agglomerated particle(s) comes into contact with a variety of compounds. Some nuclei mode particles will consist of primarily carbon spherules that did not have a chance to agglomerate and they will also contain adsorbed hydrocarbons. The vapor phase hydrocarbons are not supersaturated and will remain in the vapor state. At certain conditions during the dilution process, the $\mathrm{H}_{2} \mathrm{SO}_{4}$ vapor and the $\mathrm{H}_{2} \mathrm{O}$ vapor will undergo binary homogeneous nucleation and form $\mathrm{H}_{2} \mathrm{SO} 4-\mathrm{H}_{2} \mathrm{O}$ nuclei mode particles that are comparable in size to the nuclei mode carbon particles.

In the conditions where diesel exhaust testing parameters vary greatly, it is critical to understand how testing conditions may affect the nature of the diesel particulate matter being measured (Mostafa et al., 2001).

The key environmental or test parameters that have been identified, include:

- Dilution ratio and resulting saturation

- Time the particles spend at varying dilution ratios or sampling conditions (residence time)

- Humidity and temperature

- Background particle and gas concentration 
Emission characteristics that have a role in affecting the measured particle include:

- Size, number, and composition of particle emitted from the engine

- Composition and quantity of volatile particle precursors in the exhaust

- Fuel and oil hydrocarbon chemistry

- Fuel and oil sulfur levels

- Engine operating condition

The summary of particle dynamics including various transformation stages discussed in this chapter is presented in Table 2.1. 
Table 2.1. Summary of Particle Dynamics and Transformations Processes

\begin{tabular}{|c|c|}
\hline Process & Impact \\
\hline Nucleation & $\begin{array}{l}\text { - Homogeneous nucleation may create large numbers of new } \\
\text { particles } \\
\text { - Nucleation rates are highly nonlinear functions of saturation } \\
\text { ratio } \\
\text { - Heterogeneous nucleation leads to the growth of existing } \\
\text { particles } \\
\text { - Increases number concentration } \\
\text { - Increases volume concentration }\end{array}$ \\
\hline Particle coagulation & $\begin{array}{l}\text { - Dependent on particle size and concentration } \\
\text { - Does not affect total particle mass } \\
\text { - Causes decrease in particle number concentration and increase } \\
\text { in particle size } \\
\text { - No change in volume concentration } \\
\text { - Increase in particle size may effect loss mechanisms } \\
\text { - May affect diesel aerosols if dilution is delayed } \\
\text { - Typical time constant, } \tau=1 / \mathrm{kN}_{0} \sim 10^{9} / \mathrm{N}_{0}(\mathrm{~s}) \text { for diesel size } \\
\text { particles, } \mathrm{N}_{0}=\text { initial particle concentration }\left(1 / \mathrm{cm}^{3}\right) \text { (Fuchs, } \\
\text { 1964) }\end{array}$ \\
\hline Condensation & $\begin{array}{l}\text { - Condensation of volatile constituents will affect size and mass } \\
\text { of measured particulate matter } \\
\text { - Affected by saturation ratio and testing conditions such as : } \\
\text { - Temperature, pressure, and humidity } \\
\text { - Particle formed by nucleation may grow by condensation. }\end{array}$ \\
\hline
\end{tabular}


The next Chapter presents the theoretical formulations and governing equations used for the current work to describe the plume dispersion, binary homogeneous nucleation model for the $\mathrm{H}_{2} \mathrm{SO}_{4}-\mathrm{H}_{2} \mathrm{O}$ mixture, and the coagulation model for the accumulation mode of particulate matter formation. 


\section{CHAPTER 3}

\section{THEORETICAL FORMULATION AND GOVERNING EQUATIONS}

There are numerous mathematical models available in the published literature that have examined the dynamics and formation of particulate matter. However, there are very limited number of studies available in the literature, if any, which take into account the detailed structure of an exhaust plume. To this end, a detailed computational fluid dynamics analysis of a heavy-duty truck exhaust plume is used to predict the rate of variation of particle number concentration with the local dilution of exhaust gases in the ambient.

\subsection{PLUME DISPERSION MODEL}

An accurate and useful plume dispersion model must include the effects of turbulent mixing, convection, diffusion, temperature variations, and species transport among others. To this end, the governing transport equations for the mean fluid motion, species, and enthalpy are solved inside the wind-tunnel space using the commercial

computational fluid dynamics package FLUENT ${ }^{\mathrm{TM}}$. The mean fluid motion represented as the time averaged equations for the conservation of momentum is written as:

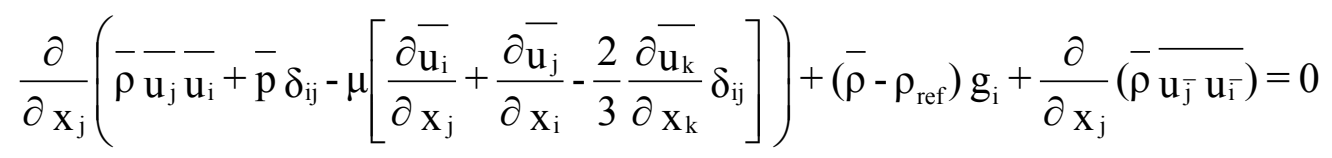

where $u_{i}$ is the velocity component in the direction of coordinate $x_{i}, \rho$ is the fluid density, $\mathrm{g}_{\mathrm{i}}$ is the magnitude of the gravitational acceleration in the $\mathrm{i}$-direction, $\mathrm{p}$ is the pressure, $\mu$ 
is the laminar viscosity, and the operator $\delta_{\mathrm{ij}}$ is unity for $\mathrm{i}=\mathrm{j}$ and zero when $\mathrm{i} \neq \mathrm{j}$. The last term (Reynolds stresses) is modeled using the "two-equation" k- $\varepsilon$ model:

$$
-\left(\bar{\rho} \overline{\mathrm{u}_{\bar{j}} \mathrm{u}_{\bar{i}}}\right)=\mu_{\mathrm{t}}\left[\frac{\partial \overline{\mathrm{u}_{\mathrm{i}}}}{\partial \mathrm{x}_{\mathrm{j}}}+\frac{\partial \overline{\mathrm{u}_{\mathrm{j}}}}{\partial \mathrm{x}_{\mathrm{i}}}-\frac{2}{3} \frac{\partial \overline{\mathrm{u}_{\mathrm{k}}}}{\partial \mathrm{x}_{\mathrm{k}}} \delta_{\mathrm{ij}}\right]
$$

where $\mu_{\mathrm{t}}$ is the turbulent viscosity that may be related to $\mathrm{k}$ and $\varepsilon$ by:

$$
\mu_{\mathrm{t}}=\mathrm{c}_{\mu} \mathrm{k}^{2} / \varepsilon
$$

where $\mathrm{c}_{\mu}$ is a constant of the model. For closure, the following differential equations for $\mathrm{k}$ and $\varepsilon$ also need to be solved:

$\frac{\partial}{\partial \mathrm{xj}_{\mathrm{j}}}\left(\bar{\rho} \overline{\mathrm{uj}_{\mathrm{j}}} \mathrm{k}\right)-\frac{\partial}{\partial \mathrm{x} \mathrm{j}_{\mathrm{j}}}\left(\Gamma \mathrm{k} \frac{\partial \mathrm{k}}{\partial \mathrm{x} \mathrm{j}_{\mathrm{j}}}\right)-\mu_{\mathrm{t}} \frac{\partial \overline{\mathrm{u}_{\mathrm{i}}}}{\partial \mathrm{x} \mathrm{j}_{\mathrm{j}}}\left(\frac{\partial \overline{\mathrm{u}_{\mathrm{i}}}}{\partial \mathrm{x}_{\mathrm{j}}}+\frac{\partial \overline{\mathrm{u}_{j}}}{\partial \mathrm{xi}_{\mathrm{i}}}\right)+\overline{\rho \varepsilon}=0$

$\frac{\partial}{\partial \mathrm{x}_{\mathrm{j}}}\left(\bar{\rho} \overline{\mathrm{u}_{\mathrm{j}}} \varepsilon\right)-\frac{\partial}{\partial \mathrm{x}_{\mathrm{j}}}\left(\Gamma_{\varepsilon} \frac{\partial \varepsilon}{\partial \mathrm{x}_{\mathrm{j}}}\right)-\mathrm{C}_{1} \frac{\varepsilon}{\mathrm{k}} \mu_{\mathrm{t}} \frac{\partial \overline{\mathrm{u}_{\mathrm{i}}}}{\partial \mathrm{x}_{\mathrm{j}}}\left(\frac{\partial \overline{\mathrm{u}_{\mathrm{i}}}}{\partial \mathrm{x}_{\mathrm{j}}}+\frac{\partial \overline{\mathrm{u}_{\mathrm{j}}}}{\partial \mathrm{x}_{\mathrm{i}}}\right)+\mathrm{C}_{2} \bar{\rho} \frac{\varepsilon^{2}}{\mathrm{k}}=0$

where $\mathrm{C}_{1}$ and $\mathrm{C}_{2}$ are constants, and $\Gamma_{\mathrm{k}}$ and $\Gamma_{\varepsilon}$ are determined via Boussinesq approximations.

In addition to the conservation of momentum, the equation of mass continuity (Equation 3.6), conservation of energy (Equation 3.7), and advection-diffusion of transport of species are also solved. 


$$
\begin{gathered}
\frac{\partial}{\partial x_{i}}\left(\bar{\rho} \overline{u_{i}}\right)=0 \\
\frac{\partial}{\partial t}(\rho h)+\frac{\partial}{\partial x_{i}}\left(\rho u_{i} h\right)=\frac{\partial}{\partial x_{i}}\left(k+k_{t}\right) \frac{\partial T}{\partial x_{i}}-\frac{\partial}{\partial x_{i}} \sum_{j-}\left(h_{\bar{j}} J_{\bar{i}^{\prime}, i}\right)+\frac{D p}{D t}+\tau_{i k} \frac{\partial u_{i}}{\partial x_{k}} \\
\frac{\partial}{\partial t}\left(\rho C_{i}\right)+\frac{\partial}{\partial x_{i}}\left(u_{i} C_{i}\right)=-\frac{\partial}{\partial x_{i}} J_{\bar{i}^{,}, i}+R_{i}
\end{gathered}
$$

where $\mathrm{h}$ is the static enthalpy, $\mathrm{k}$ is the molecular conductivity, $\mathrm{k}_{\mathrm{t}}$ is the effective conductivity due to turbulent transport $\left(\mathrm{k}_{\mathrm{t}}=\mu_{\mathrm{t}} / \mathrm{Pr}_{\mathrm{t}}\right), \mathrm{J}_{\mathrm{i}}, \mathrm{i}$ is the diffusion flux of the $\mathrm{i}^{\text {th }}$ species arising due to concentration gradients, $C_{i}$ is the mean concentration of the $i^{\text {th }}$ species, and $\mathrm{R}_{\mathrm{i}}$ is the mass rate of creation or depletion of the $\mathrm{i}^{\text {th }}$ species during chemical reaction. The influence of turbulence on the reaction rate is taken into account by employing the eddy-dissipation model which relates the rate of reaction to the rate of dissipation of the reactant and product-containing eddies as follows (Spalding, 1970):

$$
\begin{aligned}
& R_{i^{\prime}, k}=v_{i^{\prime}, k}^{\prime} M_{i^{\prime}} A \rho \frac{\varepsilon}{k} \frac{m_{R}}{v_{R, k}^{\prime} M_{R}} \\
& R_{i^{\prime}}=v_{i^{\prime}, k}^{\prime} M_{i^{\prime}} A B \rho \frac{\varepsilon}{k} \frac{\sum_{P} m_{P}}{\sum_{j^{\prime}}^{N} v_{R, k}^{\prime \prime} M_{j^{\prime}}}
\end{aligned}
$$

where $m_{P}$ represents the mass fraction of any product species, $P, m_{R}$ and $M_{R}$ represent the mass fraction and molecular weight of a particular reactant, $\mathrm{k}$ is the turbulent kinetic energy, $\varepsilon$ is the dissipation rate, $v_{i^{\prime}, \mathrm{k}}^{\prime}$ and $v_{\mathrm{R}, \mathrm{k}}^{\prime \prime}$ are the stochiometric coefficient for reactant and product respectively, $\mathrm{R}$ is the reactant species giving the smallest value of $\mathrm{Ri}^{\prime}, \mathrm{A}$ is an empirical constant equal to 4.0 , and $\mathrm{B}$ is an empirical constant equal to 0.5 . 
The transport equations arising from the various submodels described in this section are discretized by the finite volume method using a hybrid scheme. The computations were performed using second order accurate schemes in the CFD code FLUENT $^{\mathrm{TM}}$.

It is worth mentioning that the traditional approach to predicting pollutant dispersion in the atmosphere has been the use of Gaussian plume model for several years. The Gaussian model is based on a steady state assumption, and requires the flow to be in a homogeneous and stationary turbulence state. This simplified model is described in the next section.

\subsubsection{GAUSSIAN MODEL}

The Gaussian plume models, the simplest of the dispersion models, are based on the analytical solution of the transport of species advection-diffusion equation by assuming constant diffusion coefficients. Mathematically, it can be expressed as (Seinfeld and Pandis, 1997):

$$
\begin{aligned}
& \frac{\partial c}{\partial t}+\bar{u} \frac{\partial c}{\partial x}=K_{x x} \frac{\partial^{2} c}{\partial x^{2}}+K_{y y} \frac{\partial^{2} c}{\partial y^{2}}+K_{z z} \frac{\partial^{2} c}{\partial z^{2}} \\
& c(x, y, z, 0)=0 \\
& c(x, y, z, t)=0 \quad x, y \rightarrow \pm \infty
\end{aligned}
$$


Where $\mathrm{c}$ is the mean concentration of contaminant, $\mathrm{t}$ is the time, $\bar{u}$ is the mean velocity in $\mathrm{x}$-axis, $\mathrm{K}_{\mathrm{xx}}, \mathrm{K}_{\mathrm{yy}}$, and $\mathrm{K}_{\mathrm{zz}}$ is the eddy diffusivity in $\mathrm{x}$-axis, $\mathrm{y}$-axis, and z-axis, respectively. The eddy diffusivities, $\mathrm{K}_{\mathrm{xx}}, \mathrm{K}_{\mathrm{yy}}$, and $\mathrm{K}_{\mathrm{zz}}$, are generally expressed as (Pasquill, 1974):

$$
K_{x x}=\frac{\sigma_{x}^{2}}{2 t}, \quad K_{y y}=\frac{\sigma_{y}^{2}}{2 t}, \quad \text { and } K_{z z}=\frac{\sigma_{z}^{2}}{2 t}
$$

where $\sigma_{\mathrm{x}}, \sigma_{\mathrm{y}}$, and $\sigma_{\mathrm{z}}$ is the dispersion coefficient in $\mathrm{x}, \mathrm{y}$, and $\mathrm{z}$ axis, respectively, and $\mathrm{t}$ is time. The variance $\sigma_{\mathrm{y}}{ }^{2}$ and $\sigma_{\mathrm{z}}{ }^{2}$ are treated as empirical dispersion coefficients, the functional forms of which are determined by matching the Gaussian solution to data. In that way, $\sigma_{\mathrm{y}}$ and $\sigma_{\mathrm{z}}$ actually compensate for deviations from stationary, homogeneous conditions that are inherent in the assumed Gaussian distribution.

The correlations for $\sigma_{\mathrm{y}}$, and $\sigma_{\mathrm{z}}$ require knowledge of atmospheric variables that may not be available. In that case, the correlations for $\sigma_{y}$, and $\sigma_{z}$ are based on readily available data. The Pasquill-Gifford stability categories A (extremely unstable) through F (stable) provide a basis for such correlations. The most widely used $\sigma_{y}$, and $\sigma_{z}$ correlations based on the Pasquill stability classes have been those developed by Gifford (1961). In this research, $\sigma_{y}$, and $\sigma_{z}$ can be used in stability class D. Klug (1969) represented $\sigma_{\mathrm{y}}$, and $\sigma_{\mathrm{z}}$ by the power-law expression for stability class D as:

$$
\begin{aligned}
& \sigma_{y}=R_{y} x^{r_{y}}=0.219 x^{0.764} \\
& \sigma_{z}=R_{z} x^{r_{z}}=0.14 x^{0.727}
\end{aligned}
$$


In the absence of any cross-flow, the solution of concentration distribution in Equation (3.11) above the centerline of plume may be written as (Seinfeld and Pandis, 1997):

$$
\frac{c(x, 0, z)}{c_{o}}=\frac{Q}{2 \pi u \sigma_{y} \sigma_{z}} \exp \left(-\frac{z^{2}}{2 \sigma_{z}^{2}}\right)
$$

where $\mathrm{c}(\mathrm{x}, 0, \mathrm{z})$ is the concentration perpendicular to plume centerline, $\mathrm{Q}$ is the exhaust rate, $\mathrm{u}$ is the velocity of plume, $\sigma_{\mathrm{y}}$, and $\sigma_{\mathrm{z}}$ is the dispersion coefficient of $\mathrm{y}$ - and $\mathrm{z}$-axis, respectively, and $\mathrm{z}$ is the height of the plume at that time.

The concentration distribution from the above Equation (3.14) is modified to account for the physical obstacles along the negative z-axis as:

$$
\frac{c(x, 0, z)}{c_{o}}=\frac{Q}{2 \pi u \sigma_{y^{\prime}} \sigma_{z}} \exp \left(-\frac{z^{2}}{2 C_{e} \sigma_{z}^{2}}\right)
$$

where the horizontal dispersion coefficient $\left(\sigma_{y^{\prime}}=\mathrm{c}_{\mathrm{e}}{ }^{0.03} \sigma_{\mathrm{z}}\right)$ is modified by comparing the above solution with the CFD model, and $\mathrm{C}_{\mathrm{e}}$ is defined by $C_{e}=8\left(\frac{|z|}{x}\right)^{2}$.

Additionally, the species concentrations inside the plume are determined using a probability density function (PDF) mixture fraction formulation. The next section describes the CFD formulation of probability density function (PDF) mixture fraction approach. 


\subsubsection{Probability Density Function (PDF) Model}

The mixture fraction probability density function (PDF) modeling approach is based on the assumption that the instantaneous thermo-chemical state of the fluid is related to a known conserved scalar quantity, mixture fraction (f). In this approach, individual species transport equations are not solved, instead a single conserved scalar (the mixture fraction) is solved and the individual component concentrations are derived from the predicted mixture distribution. For a binary system consisting of species $\mathrm{CO}_{2}$ and $\mathrm{N}_{2}$, the mixture fraction can be written in terms of the elemental mass fraction as (Sivathanu and Faeth, 1990):

$$
f=\frac{m_{\mathrm{CO}_{2}}}{m_{\mathrm{CO}_{2}}+m_{N_{2}}}
$$

where $\mathrm{m}_{\mathrm{CO} 2}$ and $\mathrm{m}_{\mathrm{N} 2}$ are the elemental mass fractions of species $\mathrm{CO}_{2}$ and $\mathrm{N}_{2}$, respectively.

As noted above, the mixture fraction, $\mathrm{f}$, is a conserved quantity. Its value at each point in the flow domain is computed by solving the following conservation equation in the turbulent flow field:

$$
\frac{\partial}{\partial t}(\rho f)+\frac{\partial}{\partial x_{i}}\left(\rho u_{i} f\right)=\frac{\partial}{\partial x_{i}}\left(\frac{\mu_{t} \partial f}{\sigma_{t} \partial x_{i}}\right)
$$


In addition to solving for the mean mixture fraction, conservation equation for the mixture fraction variance, $\mathrm{f}^{2}($ bar) defined by Equation (3.18) is also solved:

$$
\frac{\partial}{\partial t}\left(\rho f^{\prime 2}\right)+\frac{\partial}{\partial x_{i}}\left(\rho u_{i} f^{\prime 2}\right)=\frac{\partial}{\partial x_{i}}\left(\frac{\mu_{t} \partial f^{\prime 2}}{\sigma_{t} \partial x_{i}}\right)+C_{g} \mu_{t}\left(\frac{\partial f}{\partial x_{i}}\right)^{2}-C_{d} \rho \frac{\varepsilon}{\kappa} f^{\prime 2}
$$

where the constants $\sigma_{\mathrm{t}}, \mathrm{c}_{\mathrm{g}}$ and $\mathrm{c}_{\mathrm{d}}$ take the values $0.7,2.86$, and 2.0 , respectively. The mixture fraction variance is used in the closure model describing turbulence-chemistry interactions.

The transport equations arising from the PDF described in this section are discretized by the finite volume method using a hybrid scheme. The computations were performed using second order accurate schemes in CFD code FLUENT ${ }^{\mathrm{TM}}$. Gaussian model equation was solved using an EXCEL $^{\mathrm{TM}}$ worksheet. The CFD model was applied on the detailed geometry of heavy-duty truck operating inside the wind tunnel, and the Gaussian model was however applied in a very simplified setting without considering any detailed geometry of the truck. Gaussian model is solved with the simple boundary conditions stated in Equation (3.11).

It is now worth mentioning that there are two distinct modes by which particles are formed in the atmosphere from diesel exhaust emissions. The first, nuclei mode, is brought by the build-up of concentration of condensable vapor in the atmosphere, either generated inside the cylinder, or brought in from distant sources by transport processes. The nuclei mode contributes to the majority of the particle number but does not contribute significantly to the total PM mass. The second, coagulation (or agglomeration) consists mainly of particle growth through carbonaceous agglomerates and adsorbed 
volatile compounds. These physical and chemical processes affect the size distribution of diesel engine exhaust particles. To this end, mathematical modeling is a useful approach to advance the understanding of these mechanisms of formation and resultant emissions to the environment. The next section describes the formation and growth of discrete ultra fine particles in the exhaust stream of a diesel engine.

\subsection{DISCRETE PARTICLE DYNAMICS}

The evolution of the particle size distribution due to coagulation, nucleation and coagulation is represented by the following discrete dynamical equation (Sienfeld and Pandis, 1997):

$$
\frac{\partial C_{k}}{\partial t}=\frac{1}{2} \sum_{j=1}^{k-1} \underbrace{\beta_{k-j, j} C_{k-j} C_{j}-C_{k} \sum_{j=1}^{\infty} \beta_{k, j} C_{j}}_{\text {Coagulation }}+\underbrace{J(t) \delta(k)+\beta_{1, k-1} C_{1} C_{k-1}-\beta_{1, k} C_{1} C_{k}}_{\text {Nucleation }}
$$
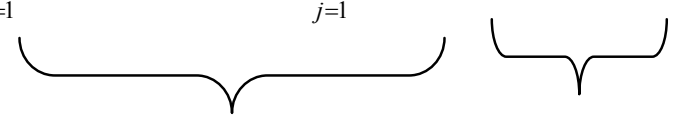

where $C_{k}$ is time (t)-dependent number concentration $\left(\right.$ No. $\mathrm{cm}^{-3}$ ) of particles of volume $v_{k}$ $\left(\mathrm{cm}^{3}\right), \beta$ is the coagulation kernel $\left(\mathrm{cm}^{3} \mathrm{No}^{-1} \mathrm{~s}^{-1}\right)$ of two colliding particles, $\mathrm{J}(\mathrm{t})$ is the nucleation rate and $\delta$ is the Kronecker's delta with the value equal to 1 for the $\mathrm{k}^{\text {th }}$ bin of volume $\mathrm{v}_{\mathrm{k}}$; and 0 otherwise. The first term on right hand side of Equation (3.19) indicates that a particle of volume $\mathrm{v}_{\mathrm{k}}$ can only come into existence if two particles with volumes $\mathrm{v}_{\mathrm{j}}$ and $v_{k-j}$ collide. The one-half is required so that each combination is counted only once. 
Many recent studies have shown that the diesel particulate matter (PM) remains in a continuous transforming (unstable) state for some time after it is emitted into the atmosphere. This is in part due to the continuation of in-stack coagulation and adsorption, but also due to the significant quantities of condensable organics and inorganics usually present in diesel exhaust. The fate of these condensable organics/inorganics is significantly affected by the dilution and atmospheric aging of the exhaust stream. A number of processes occur during atmospheric aging that can alter the size distribution of particulate matter (PM) including binary homogeneous nucleation, coagulation and condensation. Homogeneous nucleation is the spontaneous formation of a nuclei or nanosized particles (nano-particles) from volatile material within a locally supersaturated zone. Binary homogeneous nucleation requires at least two components interacting to form particles. In the case of diesel exhaust, the two components that most likely would nucleate are $\mathrm{H}_{2} \mathrm{SO}_{4}$ and water vapor. The formation of particles from this binary homogeneous nucleation is described in the following section.

\subsubsection{Nucelation Model}

The $\mathrm{H}_{2} \mathrm{SO}_{4}$ hydrate (embryo) formation rate is predicted by the equation (Reiss, 1950)

$$
J=C \exp \left(-\Delta G * / k_{b} T\right)
$$

where $\mathrm{C}$ is frequency factor, $\mathrm{k}_{\mathrm{b}}$ is Boltzmann's constant, $\mathrm{T}$ is the temperature and $\Delta \mathrm{G}^{*}$ is the free energy required to form an embryo. The free energy of formation of an embryo of arbitrary size and composition is (Roedel, 1979)

$$
\Delta G=n_{1}\left(\mu_{11}-\mu_{1 g}\right)+n_{2}\left(\mu_{21}-\mu_{2 g}\right)+4 \pi r^{2} \sigma
$$


where $\mathrm{n}_{1}$ and $\mathrm{n}_{2}$ are the number of molecules of component water and $\mathrm{H}_{2} \mathrm{SO}_{4}$ in the embryo, $\mu_{11}$ and $\mu_{21}$ are the chemical potentials of water and $\mathrm{H}_{2} \mathrm{SO}_{4}$ taken for a macroscopic amount of a liquid phase of the same composition, $\mu_{1 \mathrm{~g}}$ and $\mu_{2 \mathrm{~g}}$ are the chemical potentials of components water and $\mathrm{H}_{2} \mathrm{SO}_{4}$ in the gas phase, and $\sigma$ is the surface tension of the binary mixture.

$\Delta \mathrm{G}^{*}$ can be found by solving the two equations

$$
\left(\frac{\partial \Delta G}{\partial n_{1}}\right)_{n_{2}}=0, \quad\left(\frac{\partial \Delta G}{\partial n_{2}}\right)_{n_{1}}=0
$$

Solving Equations (3.22), one obtains

$$
\begin{aligned}
& \Delta \mu_{i}+\frac{2 \sigma v_{i}}{r^{*}}=0 \quad(\mathrm{i}=1,2) \\
& v_{2} \Delta \mu_{1}=v_{1} \Delta \mu_{2}
\end{aligned}
$$

where $v_{1}$ and $v_{2}$ are the partial molar volumes. $r^{*}$ is the radius of the droplet, and $4 \pi *^{* 3} / 3=n_{1} v_{1}+n_{2} v_{2}$

\subsubsection{Hydration Effects on Nucleation}

Sulfuric acid has an affinity to form hydrates in the gas phase, which affect sulfuric acid vapor activity. The chemical potential difference of $i$ with the help of liquid and vapor activities (Kulmala et al., 1998) is expressed as:

$$
\Delta \mu_{i}=-k T \ln \frac{A_{i v}}{A_{i l}}
$$

where the activities are given by $\mathrm{A}_{\mathrm{il}}=\mathrm{p}_{\mathrm{i}, \mathrm{sol}} / \mathrm{p}_{\mathrm{i}, \mathrm{s}}$ and $\mathrm{A}_{\mathrm{iv}}=\mathrm{p}_{\mathrm{i}} / \mathrm{p}_{\mathrm{i}, \mathrm{s}}$, and $\mathrm{p}_{\mathrm{i}}, \mathrm{p}_{\mathrm{i}, \mathrm{s}}$ and $\mathrm{p}_{\mathrm{i}, \text { sol }}$ denote partial pressure, saturation vapor pressure and vapor pressure over the solution surface, respectively. 
The hydration correction for the acid activities has the following form:

$$
\left(\ln \frac{A_{a v}}{A_{a l}}\right)_{c o r r}=\ln \frac{A_{a v}}{A_{a l}}+\ln F .
$$

The hydration correction factor $\mathrm{F}$ is given by

$$
F=\left[\frac{1+K_{1} p_{w, s o l}+\cdots+K_{1} K_{2} \times \cdots \times K_{k} p_{w, s o l}^{k}}{1+K_{1} p_{w}+\cdots+K_{1} K_{2} \times \cdots \times K_{k} p_{w}^{k}}\right]^{n_{a}}
$$

where the subscripts $\mathrm{w}$ and a refer to water and acid, respectively, and $\mathrm{K}_{\mathrm{h}}$ is the equilibrium constant for formation of hydrate containing $\mathrm{h}$ water molecules. In this research, it is assumed that the concentration of hydrates containing more than 10 water molecules is negligible, that is, $\mathrm{k}=10$.

Jaecker-Voirol et al. (1987) gave an approximate expression for the equilibrium constants:

$$
\ln K_{h}=\ln \left(A_{w v} / A_{w l}\right)-2 \sigma v_{w} /\left(k T r_{h}\right)
$$

where $r_{h}$ denotes the radius of the hydrate and $v_{w}$ is the partial molecular volume of water.

Frequency factor $\mathrm{C}$ is defined as follows:

$$
C=N_{v} A B_{A V} Z
$$

where $\mathrm{N}_{\mathrm{v}}=\mathrm{N}_{\mathrm{wv}}+\mathrm{N}_{\mathrm{av}}+\sum_{\mathrm{h}=1,10} \mathrm{~N}_{\mathrm{h}}$ is the total number density of the vapor phase, $\mathrm{A}$ is the surface area of the nucleus, $\mathrm{B}_{\mathrm{AV}}$ is the average growth rate, and $\mathrm{Z}$ is Zeldovich nonequilibrium factor. In this research, it has been assumed that the vapor consists of water monomers, sulfuric acid monomers, and hydrates containing 1 acid and 1 to 10 water molecules. The average growth rate is then given by 


$$
B_{A V}=\frac{B_{w} B_{a}-\left(\sum_{h} B_{h}\right)^{2}}{B_{w} \sin ^{2} \theta+B_{a} \cos ^{2} \theta-2 \sum_{h} B_{h} \sin \theta \cos \theta}
$$

where $\theta$ is the angle between the $\mathrm{n}_{\mathrm{w}}$ axis and direction of growth at the saddle point in three-dimensional space $\left(\mathrm{n}_{\mathrm{w}}, \mathrm{n}_{\mathrm{a}}, \Delta \mathrm{G}\right)$. The condensation rates of the monomers are obtained from kinetic theory:

$$
B_{i}=N_{i v} \sqrt{\frac{k_{B} T}{2 \pi}\left(\frac{1}{m_{i}}-\frac{1}{m *}\right)} \quad(\mathrm{i}=\mathrm{w}, \mathrm{a})
$$

where $m_{i}$ is the molecular mass of species $i$ and $m^{*}$ is the mass of the critical nucleus. The condensation rates of the hydrates are given by

$$
B_{h}=N_{h} \sqrt{\frac{k_{B} T}{2 \pi}\left(\frac{1}{m_{h}}-\frac{1}{m^{*}}\right)} \quad(\mathrm{h}=1, \cdots, 10)
$$

and $\mathrm{m}_{\mathrm{h}}=\mathrm{m}_{\mathrm{a}}+\mathrm{hm}_{\mathrm{w}}$.

The growth angle $\theta$ by the angle of steepest descent is approximated by:

$$
\tan \theta \approx \frac{x_{a l}}{x_{w l}} .
$$

The Zeldovich factor is calculated from the equation of Kulmala and Viisanen (1991), who considered a critical nucleus consisting of fictitious "average" monomers with volume $\mathrm{V}_{\mathrm{AB}}=\mathrm{X}_{\mathrm{wl}} \mathrm{V}_{\mathrm{w}}+\mathrm{x}_{\mathrm{al}} \mathrm{V}_{\mathrm{a}}$, in which case the problem reduces to one-component nucleation:

$$
Z=\sqrt{\frac{\sigma}{k_{B} T}} \frac{v_{A V}}{2 \pi r^{* 2}} .
$$

where $\sigma$ is the surface tension, $\mathrm{k}_{\mathrm{B}}$ is the Boltzmann constant, $\mathrm{T}$ is the temperature, $\mathrm{v}_{\mathrm{av}}$ is the average volume, and $\mathrm{r}^{*}$ is the critical radius. It is known that various approximations in the pre-exponential of Equation (3.33) can produce surprisingly large errors in the 
calculated nucleation rate (see Kulmala and Laaksonen, 1990). However, Viisanen et al. (1997) suggested that the error produced by the growth angle and Zeldovich factor expressions employed here result in no more than 1 order of magnitude error in nucleation rate.

\subsubsection{Physical Parameters Used in the Models}

Partial molar free energies at $25{ }^{\circ} \mathrm{C}$ have been taken from the compilation of Giaque et al. (1960) for the sulfuric acid-water system. The values of partial molar free energy (chemical potential) for both components versus mole fraction of sulfuric acid used in this research were taken from an eighth order polynomial curve fit by Shi and Harrison (1999). Partial molar volumes were calculated from the data on density and composition given in the Chemical Engineer's Handbook (1973). Surface tension of the mixture used was based on an eighth order polynomial fit by Sabinina and Terpugow (1935). The analytical pure sulfuric acid vapor pressure equation given by Ayers et al. (1980) was used in this study:

$$
\ln p_{H_{2} \mathrm{SO}_{4}}^{s}=-10156 / T+16.259
$$

where $p_{\mathrm{H}_{2} \mathrm{SO}_{4}}^{s}$ is a saturation pressure of $\mathrm{H}_{2} \mathrm{SO}_{4}$, and $\mathrm{T}$ is temperature.

The next section describes the theory of coagulation that deals with the process of adhesion or agglomeration of particles when they come in contact with one another. The aim of this theory is to describe the particle size distribution as a function of time and space as particles collide with each other. 


\subsection{COAGULATION MODEL}

The semi-implicit solution to the coagulation equation for particles of uniform composition is presented in this section. To derive the semi-implicit solution, start with an integro-differential equation (Muller, 1928)

$$
\frac{\partial C_{v}}{\partial t}=\frac{1}{2} \int_{0}^{v} \beta_{v-\bar{v}} C_{v-\bar{v}} C_{\bar{v}} d \bar{v}-C_{v} \int_{0}^{\infty} \beta_{v, \bar{v}} C_{\bar{v}} d \bar{v}
$$

where $\mathrm{C}$ is time-dependent number concentration $\left(\right.$ No. $\left.\mathrm{cm}^{-3}\right)$ of particle of volume $\mathrm{v},(\mathrm{v}$ $\bar{v}$ ), or $\bar{v}$ (dummy integral variable of Equation (3.35), and $\beta$ is the coagulation kernel $\left(\mathrm{cm}^{3} \mathrm{No}^{-1} \mathrm{~s}^{-1}\right)$ of two colliding particles. The first integral in Equation (3.35) expresses the fact that each particle of volume $\mathrm{v}$ disappears from the interval $\mathrm{v}$ to $\mathrm{d}+\mathrm{dv}$ after colliding with a particle of volume $\bar{v}$. The second integral says that a particle of volume $\mathrm{v}$ can only come into existence if two particles with volumes $\bar{v}$ and $(\mathrm{v}-\bar{v})$ collide. The one-half is required so that each combination is counted only once. For size bins consisting of monomers (e.g. the volume of bin $\mathrm{k}$ equals $\mathrm{k} x$ the volume of bin one), rewriting Equation (3.35) as (Jacobson et al., 1994):

$$
\frac{\partial C_{k}}{\partial t}=\frac{1}{2} \sum_{j=1}^{k-1} \beta_{k-j, j} C_{k-j} C_{j}-C_{k} \sum_{j=1}^{\infty} \beta_{k, j} C_{j}
$$

where the volume subscripts in equation (3.35) are substituted with the size-bin subscripts in the Equation (3.36).

The next step in the derivation of the semi-implicit solution is to write equation (3.36) in fully implicit finite difference form as

$$
C_{k}^{t+1}=C_{k}^{t}+\frac{1}{2} \Delta t \sum_{j=1}^{k-1} P_{k, j}-\Delta t \sum_{j=1}^{\infty} L_{k, j}
$$


where $\Delta \mathrm{t}$ is the time-step (s), superscripts $\mathrm{t}$ and $\mathrm{t}+1$ indicate initial and final concentrations, respectively, while

$$
P_{k, j}=\beta_{k-j, j} C_{k-j}^{t+1} C_{j}^{t+1}
$$

and

$$
L_{k, j}=\beta_{k, j} C_{k}^{t+1} C_{j}^{t+1}
$$

are production and loss rates $\left(\right.$ No. $\left.\mathrm{cm}^{-3} \mathrm{~s}^{-1}\right)$, respectively.

Third, to obtain the semi-implicit solution, redefine the loss term from Equation (3.39) in semi-implicit form as

$$
L_{k, j}=\beta_{k, j} C_{k}^{t+1} C_{j}^{t}
$$

Using equation (3.40) instead of Equation (3.39) will allow a non-iterative solution to coagulation that approximates an exact solution. Equation (3.38) and (3.39) require that $\mathrm{P}_{\mathrm{k}, \mathrm{j}}=\mathrm{L}_{\mathrm{k}-\mathrm{j}, \mathrm{j}}$ for each $\mathrm{k}$ and $\mathrm{j}$. Applying this equality to Equation (3.40) and substituting the result and Equation (3.40) into Equation (3.37), the semi-implicit coagulation solution for monomer particles of uniform composition is obtained as:

$$
C_{k}^{t+1}=\frac{C_{k}^{t}+\frac{1}{2} \Delta t \sum_{j=1}^{k-1} \beta_{k-j, j} C_{k-j}^{t+1} C_{j}^{t}}{1+\Delta t \sum_{j=1}^{\infty} \beta_{k, j} C_{j}^{t}}
$$

where $\mathrm{k}$ varies from one to infinity. While Equation (3.41) correctly accounts for the reduction in particle number when two particles coagulate (reducing the number by onehalf), it does not conserve volume (Equation (3.37) correctly accounts for both number and volume, but is fully implicit). In order to conserve volume (which coagulation 
physically does) while giving up some accuracy in number, Jacobson et al. (1994) reformed Equation (3.41) as

$$
v_{k} C_{k}^{t+1}=\frac{v_{k} C_{k}^{t}+\Delta t \sum_{j=1}^{k-1} \beta_{k-j, j} v_{k-j} C_{k-j}^{t+1} C_{j}^{t}}{1+\Delta t \sum_{j=1}^{\infty} \beta_{k, j} C_{j}^{t}}
$$

Equation (3.42) satisfies the volume-conservation requirement, $\mathrm{v}_{\mathrm{k}-\mathrm{j}} \mathrm{P}_{\mathrm{k}, \mathrm{j}}=\mathrm{v}_{\mathrm{k}-\mathrm{j}} \mathrm{L}_{\mathrm{k}-\mathrm{j}, \mathrm{j}}$ for each $\mathrm{k}$ and $\mathrm{j}$.

While Equation (3.42) solves the equation over a monomer size-bin structure, it can be modified to solve over different bin structures. This may be done by setting up geometric bins, where the volume of one bin equals the volume of the previous bin multiplied by a constant factor. In this particular distribution, the volume of the smallest bin is $v_{1}=(4 / 3) \pi\left(r_{1}\right)^{3}$, where $r_{1}$ is the radius of the smallest bin. Furthermore, $V_{\text {RAT }}=v_{i+1} / v_{i}$ is the volume ratio of two adjacent bins, and $\mathrm{N}_{\mathrm{B}}$ is the total number of size bins. For any volume of $\mathrm{V}_{\mathrm{RAT}}$ greater than one, the volume of bin $\mathrm{i}$ is

$$
v_{i}=v_{1} V_{R A T}^{i-1}
$$

and the radius is

$$
r_{i}=r_{1} V_{R A T}^{(i-1) / 3}
$$

Consequently, the number of bins that covers the particle size range from radius $r_{1}$ to $r_{i}$ is

$$
i=1+\ln \left[\left(r_{i} / r_{1}\right)^{3}\right] / \ln \left[V_{R A T}\right] .
$$

Thus, for a radius from $0.01 \mu \mathrm{m}\left(10^{-6} \mathrm{~cm}\right)$ to $1 \mathrm{~mm}\left(10^{-1} \mathrm{~cm}\right)$, the model requires $87\left(=\mathrm{N}_{\mathrm{B}}\right)$ bins when $V_{\text {RAT }}=1.5$ and 26 bins when $V_{\text {RAT }}=4$. 
With this new bin structure, each time a model particle of size i collides and sticks to a model particle of size $\mathrm{j}$, the resulting volume of the intermediate particle is

$$
V_{i, j}=v_{i}+v_{j}
$$

The intermediate particle has volume between those of two model bins, $\mathrm{k}$ and $\mathrm{k}+1$. Defining an intermediate partition between the two bins by defining $\mathrm{f}_{\mathrm{i}, \mathrm{j}, \mathrm{k}}$, and the volume fraction of intermediate particles of size by $\mathrm{V}_{\mathrm{i}, \mathrm{j}}$, it can be mathematically written as:

$$
f_{i, j, k}=\left\{\begin{array}{lll}
\left(\frac{v_{k+1}-V_{i, j}}{v_{k+1}-v_{k}}\right) & v_{k} \leq V_{i, j}<v_{k+1} ; & k<N_{B} \\
1-f_{i, j, k-1} & v_{k-1}<V_{i, j}<v_{k} ; & k>1 \\
1 & V_{i, j} \geq v_{k} & k=N_{B} \\
0 & \text { all other cases } &
\end{array}\right.
$$

The fractions in Equation (3.47) are independent of the size-bin structure. Thus, they work with monomer structures (where all values of $\mathrm{f}$ would be 1 or 0 ), geometric structures, or random structures. Using Equation (3.47) in Equation (3.42), the general formula for volume-conserving, semi-implicit coagulation for particles of uniform composition can be written as

$$
v_{k} C_{k}^{t+1}=\frac{v_{k} C_{k}^{t}+\Delta t \sum_{j=1}^{k}\left\{\sum_{i=1}^{k-1} f_{i, j, k} \beta_{i, j} v_{i} C_{i}^{t+1} C_{j}^{t}\right\}}{1+\Delta t \sum_{j=1}^{N_{B}}\left(1-f_{i, j, k}\right) \beta_{k, j} C_{j}^{t}}
$$

In Equation (3.48), values for $\mathrm{f}_{\mathrm{i}, \mathrm{j}, \mathrm{k}}$ are frequently zero; thus, to speed the computer solution to Equation (3.48), every multiplication by a zero value of $\mathrm{f}$ is eliminated. Also in Equation (3.48) each $\mathrm{C}_{\mathrm{t}+1}$ term on the right hand side of the equation is final concentration calculated for a previous bin. No production occurs in the first bin, $\mathrm{k}=1$, since k-1 $=0$ in Equation (3.48). Thus all $C_{i}^{t+1}$ terms are known when calculating $C_{k}^{t+1}$. 
The effect of local mixing/dilution of particulate matter in ambient air is incorporated implicitly through the coagulation kernel $(\beta)$, and explicitly by dividing the right hand side of Equation (3.48) with the local dilution ratio at any given spatial location. In the present study to test the coagulation model, simple coagulation kernel is defined as

$$
\beta_{i, j}=\frac{8 k_{b} T}{3 \mu}
$$

where $\mathrm{k}_{\mathrm{b}}$ is the Boltzman constant, $\mathrm{T}$ is the absolute temperature and $\mu$ is the dynamic viscosity. However, there are various coagulation kernels available elsewhere in the literature (Tambour and Seinfeld, 1980). The variation in turbulent viscosity is strongly dependent on the ambient and diesel exhaust flow rates, and hence it is implicitly affected by the local dilution of the exhaust stream. The turbulent viscosity is predicted using the k- $\varepsilon$ turbulent closure from a CFD simulation (Kim et al., 2001). Equation (3.48), which accounts for the effect of local dilution ratio explicitly, can finally be written as:

$v_{k} C_{k}^{t+1}=\left(\frac{v_{k} C_{k}^{t}+\Delta t \sum_{j=1}^{k}\left\{\sum_{i=1}^{k-1} f_{i, j, k} \beta_{i, j} v_{i} C_{i}^{t+1} C_{j}^{t}\right\}}{1+\Delta t \sum_{j=1}^{N_{B}}\left(1-f_{i, j, k}\right) \beta_{k, j} C_{j}^{t}}\right) \frac{1}{\text { Dilution Ratio }}$

The above equation is solved to predict the concentration variation of particulate matter in the exhaust plume of a diesel truck operating at highway speeds. 
To account for the simultaneous effects of nucleation, coagulation, and condensation, the numerator of Equation (3.50) is modified to include the nucleation and condensation terms to give Equation (3.51):

$$
v_{k} C_{k}^{t+1}=\left(\frac{v_{k} C_{k}^{t}+\Delta t \sum_{j=1}^{k}\left\{\sum_{i=1}^{k-1} f_{i, j, k} \beta_{i, j} v_{i} C_{i}^{t+1} C_{j}^{t}\right\}+\Delta t v_{k} J(t) \delta\left((k)+\Delta t v_{k} \beta_{1, k-1} C_{1}^{t+1} C_{k-1}^{t+1}\right.}{1+\Delta t \sum_{j=1}^{N_{B}}\left(1-f_{i, j, k}\right) \beta_{k, j} C_{j}^{t}+\Delta t \beta_{k, j} C t_{1}}\right) \frac{1}{\text { Dilution Ratio }}
$$

The advantage of using a semi-implicit equation, such as Equations (3.41), (3.42), or (3.48) instead of a fully implicit equation, such as Equation (3.35), is significant. For example, Equations (3.41), (3.42), and (3.48) allow immediate, volumeconserving solutions. It should be mentioned here that by increasing the resolution of the bin structure (for example, by decreasing $\mathrm{V}_{\mathrm{RAT}}$ ), the error in number approaches zero while the solution remains non-iterative and volume conserving.

To test the above coagulation model (Equation 3.50) in the absence of any nucleation and condensation terms, the numerical solution is compared with the analytical solution given by Smoluchowski (1918) for a simple initial condition. For a given initial number concentration $C^{t}$ in the first bin of volume $v_{1}$, Smoluchowski (1918) derived the concentration variation at any time $t+1$ in bin of volume $v_{k}$ to be (Sienfeld and Pandis, 1997):

$$
C_{k}^{t+1}=\frac{C^{t}\left(0.5 \Delta t \beta C^{t}\right)^{k-1}}{\left(1+0.5 \Delta t \beta C^{t}\right)^{k+1}}
$$




\subsubsection{Coagulation kernel}

For part of this work, it is proposed that the coagulation kernel be calculated by assuming only Brownian diffusion. However, empirical equations in Pruppacher and Klett (1978) described additional coagulation rates, including those for convective diffusion enhancement, gravitation, turbulent shear, and turbulent inertial motion (See also Saffman and Turner (1956) for coagulation rates due to turbulent shear and inertial motion). To calculate the Brownian diffusion kernel $\left(\beta_{i, j}^{B}-\mathrm{cm}^{3} \mathrm{No}^{-1} \mathrm{~s}^{-1}\right)$ it is proposed that Fuchs' (1964) interpolation formula be used:

$$
\beta_{i, j}^{B}=\frac{4 \pi\left(r_{i}+r_{j}\right)\left(D_{i}+D_{j}\right)}{\frac{r_{i}+r_{j}}{r_{i}+r_{j}+\left(\delta_{i}^{2}+\delta_{j}^{2}\right)^{1 / 2}}+\frac{4\left(D_{i}+D_{j}\right)}{\left(\bar{v}_{p i}^{2}+\bar{v}_{p j}^{2}\right)^{1 / 2}\left(r_{i}+r_{j}\right)}}
$$

where $r_{i}$ and $r_{j}$ are the radii $(\mathrm{cm})$ of particles $i$ and $j$, respectively. Also, $D_{i}\left(\right.$ or $\left.D_{j}\right)$ is the particle diffusion coefficient $\left(\mathrm{cm}^{2} \mathrm{~s}^{-1}\right)$ defined as

$$
D_{i}=\frac{k_{B} T}{6 \pi r_{i} \eta}\left(\frac{5+K n_{i}+6 K n_{i}^{2}+18 K n_{i}^{3}}{5-K n_{i}+(8+\pi) K n_{i}^{2}}\right)
$$

which simplifies to the Stokes-Einstein formula in the continuum regime $\left(\mathrm{Kn}_{\mathrm{i}} \ll 1\right)$. In Equation (3.54), $K n_{i}=\lambda_{g} / r_{i}$ is the Knudsen number of particle i, $k_{B}$ is Boltzmann's constant, $\mathrm{T}$ is the temperature $(\mathrm{K})$, and $\eta$ is the dynamic viscosity of air.

In addition, the mean free path $\left(\mathrm{cm} \mathrm{s}^{-1}\right)$ of a gas molecule is

$$
\lambda_{g}=\frac{2 \eta}{\rho_{g} \bar{v}_{g}}
$$

where $\rho_{\mathrm{g}}$ is the density of air, and $\bar{v}_{g}$ is the mean thermal velocity of an air molecule. The thermal velocity of an air molecule is similar to that of a particle of size $i$ 


$$
\bar{v}_{g} \quad \text { or } \quad \bar{v}_{p i}=\left(\frac{8 k_{B} T}{\pi m_{i}}\right)^{1 / 2}
$$

except that, for a particle of size $\mathrm{i}, \mathrm{m}_{\mathrm{i}}$ is the mass of the particle, and for an air molecule, $\mathrm{m}_{\mathrm{i}}$ is the mass of the air molecule. Finally, Fuchs (1964) defined the mean distance from the center of a sphere and traveling a distance of particle mean free path $\lambda_{\text {pi }}$ as

$$
\delta_{i}=\frac{\left\{\left(2 r_{i}+\lambda_{p i}\right)^{3}-\left(4 r_{i}^{2}+\lambda_{p i}^{2}\right)^{3 / 2}\right\}}{6 r_{i} \lambda_{p i}}-2 r_{i}
$$

where

$$
\lambda_{p i}=\frac{8 D_{i}}{\pi \bar{v}_{p i}}
$$

In the continuum regime, Equation (3.53) simplifies to its numerator, while in the free molecular regime $\left(\mathrm{Kn}_{\mathrm{i}} \gg 10\right)$ it simplifies to

$$
\beta_{i, j}^{B}=\pi\left(r_{i}+r_{j}\right)^{2}\left(\bar{v}_{p i}^{2}+\bar{v}_{p j}^{2}\right)^{1 / 2}
$$

Equations arising from the various submodels described in this section are discretized using the semi-implicit finite difference scheme. The predicted results from the CFD models are compared with the experimental data for $\mathrm{CO}_{2}$ concentration, dilution ratio, and temperature variations inside the plume. The numerical prediction of particulate matter size distribution and concentration using the above sub-models is also presented, discussed and compared with the experimental data for PM size distributions and concentrations in the next Chapter. 


\section{CHAPTER 4}

\section{RESULTS AND DISCUSSION}

The transport equations arising from the various submodels described in this section have been discretized by the finite volume method using a hybrid scheme. The computations have been performed using second order accurate schemes in the CFD code FLUENT $^{\mathrm{TM}}$.

\subsection{SIMULATION CONDITIONS}

The geometry of the heavy-duty on-road tractor truck powered by a diesel engine rated at $330 \mathrm{hp}$, which was used during a NASA Langley wind tunnel has been discretized using approximately 500,000 hexahedral and tetrahedral control volumes (cells) as shown in Figures (4.1) and (4.2). The dimensions of the truck were $28 \mathrm{ft}(8.4 \mathrm{~m})$ long, $8 \mathrm{ft}(2.44 \mathrm{~m})$ wide and $13 \mathrm{ft}(4 \mathrm{~m})$ high, and the dimensions of the wind-tunnel test section used in the current simulation were $90 \mathrm{ft}(27.4 \mathrm{~m}) \times 60 \mathrm{ft}(18.3 \mathrm{~m}) \times 30 \mathrm{ft}(9.1 \mathrm{~m})$. The air velocity provided by fans in the wind tunnel was approximately $24.6 \mathrm{~m} / \mathrm{s}$ which simulated the situation of a truck traveling at $55 \mathrm{mph}$ on a highway. The exhaust was emitted out at $29.8 \mathrm{~m} / \mathrm{s}$ through a $5 "(0.13 \mathrm{~m})$ diameter stack behind the truck's cabin (more detailed discussion of the experimental conditions may be found in Boyce et al. (2000) and Gautam et al. (2000)). The $\mathrm{CO}_{2}$ concentration in the raw exhaust (undiluted exhaust) was $6 \%$ under the steady state operation of $55 \mathrm{mph}$. The gas flow in the above configuration is described by the time-averaged equations of global mass, momentum, enthalpy and species mass fractions. The standard k- $\varepsilon$ turbulence closure and finite rate chemistry/eddy dissipation has been used in the current CFD simulation. 


\subsection{PLUME PREDICTIONS}

To test the model, simulation results were compared to measurements of relative $\mathrm{CO}_{2}$ concentration, temperature variations, and dilution ratios inside a dispersing plume emanating from the truck's exhaust pipe inside the Langley wind tunnel. These variations were actually averaged concentration and temperature values obtained at a predetermined sampling rate and time. Data were collected at the several locations perpendicular to the direction of flow inside the plume, and at certain distances downstream of the source along the centerline of the plume. The relative concentration $\left(\mathrm{R}_{\mathrm{C}}\right)$ of carbon dioxide has been defined as the ratio of the $\mathrm{CO}_{2}$ concentration at a given location $\mathrm{C}(\mathrm{x}, \mathrm{y}, \mathrm{z})$ (in the diluted exhaust; the plume) to raw exhaust $\mathrm{CO}_{2}$ concentration $\left(\mathrm{C}_{0}\right)$ :

$$
R_{C}=\frac{C(x, y, x)-\text { Background Concentration }}{C_{o}-\text { Background Concentration }}
$$

The ambient $\mathrm{CO}_{2}$ concentration or background concentration inside the recirculating wind tunnel was measured to be equal to $640 \mathrm{ppm}$, and $\mathrm{C}_{\mathrm{o}}$, the raw $\mathrm{CO}_{2}$ concentration from the exhaust pipe was $6 \%(60,000 \mathrm{ppm})$ by volume. The relative concentration defined by Equation (4.1) has been plotted in Figure 4.3 on the centerline plume. It may be seen from the Figure 4.3 that a significant amount of exhaust gases were trapped in the re-circulation region below the wind deflector (drag reducing air shield; see Figure 4.1 for location). It is worth mentioning that such an effect can not be accurately modeled via a Gaussian plume model defined by Equation (4.1), which is traditionally used in the application of atmospheric dispersion modeling in the far field regions from the source. The CFD models provide valuable tools for formulating the physical phenomena important to plume dispersion, for example to include the effects of 
turbulent mixing, convection, diffusion, temperature gradients, and species transport among others. The concentration variation originating from the exhaust pipe, representing the relative concentration $\left(\mathrm{R}_{\mathrm{C}}\right)$ is plotted in Figure 4.4. These concentration variations also depict the growth and the bounds for dispersion of the plume. It may be seen from Figure 4.4 that the center of the plume is pointing slightly downward, and this maybe attributed to the wake effects behind the truck.

The relative concentration $\left(\mathrm{R}_{\mathrm{c}}\right)$ predicted by the CFD model is compared with the experimental data measured along the centerline of the plume as shown in Figure 4.5. It may be seen that the relative concentration of $\mathrm{CO}_{2}$ drops rapidly close to the ambient concentration value of the $\mathrm{CO}_{2}$ within a distance of 100 inches $(2.54 \mathrm{~m})$ downstream of the exhaust stack outlet. This is due to the fact that the small flow rate of $13.4 \mathrm{ft}^{3} / \mathrm{s}(0.38$ $\left.\mathrm{m}^{3} / \mathrm{s}\right)$ coming out of the exhaust pipe is turbulently mixing with the $126,358 \mathrm{ft}^{3} / \mathrm{s}(3,578$ $\mathrm{m}^{3} / \mathrm{s}$ ) of ambient air. The small variations along the centerline predicted by the CFD model are due to high amount of turbulence present near the exhaust pipe.

The velocity vectors predicted from the CFD simulations on a plane passing through the center of the exhaust pipe are shown in Figure 4.6. It may be seen that the velocity vectors (or flow field) in the far field are fairly uniform, and not surprisingly, there is a significant recirculation of the flow below the wind deflector that extends some distance downstream of the truck. Accurate prediction of the recirculation is important for accurately predicting the relative concentration of $\mathrm{CO}_{2}$ inside the plume. In the CFD 
models, the dispersion coefficients are not necessarily constant, but they do include the effect of turbulent mixing (or eddies) in the dispersion of the plume.

The raw $\mathrm{CO}_{2}$ concentration inside the dispersion plume predicted by the CFD simulations is compared with the experimentally measured values at a few locations downstream of the exhaust pipe in Figure 4.7. The predicted and measured $\mathrm{CO}_{2}$ values on a plume centerline are plotted at $20 "(0.51 \mathrm{~m}), 40$ " $(1.02 \mathrm{~m}), 80$ " $(2.03 \mathrm{~m})$ and 120 " $(3.05 \mathrm{~m})$ downstream of the exhaust pipe. At each of these 4 locations, raw $\mathrm{CO}_{2}$ concentrations are plotted to show their variation along a vertical line that is perpendicular to the centerline of the plume. It may be seen that the $\mathrm{CO}_{2}$ concentration diffuses close to the ambient $\mathrm{CO}_{2}$ concentration $(640 \mathrm{ppm})$ within a vertical distance of approximately $\pm 30 "(0.76 \mathrm{~m})$ from the centerline of the plume. Also, a slight asymmetry of the various curves may be observed about the abscissa (centerline) of the plume. This is due to the fact that plume decays very quickly in the absence of any physical obstacle above the centerline. However, in the presence of physical walls (obstacles) the plume decays more slowly below the centerline. The re-circulation of air flow is also enhanced below the top arch wall (see Figure 4.1 for location of wind deflector) due to an opening below the back cabin which brings the under carriage air. It was noted from an additional exploratory simulation in the absence of any physical walls near the plume source, and without any undercarriage flow, the $\mathrm{CO}_{2}$ concentration was approximately $5-8 \%$ higher than the current simulation at 20 " $(0.508 \mathrm{~m})$ downstream of the source. The difference in $\mathrm{CO}_{2}$ concentration from above two cases is not significant at a distance far away from the plume source. It is evident from Figure 4.7 that the $\mathrm{CO}_{2}$ concentration predicted by the 
CFD model agrees very well with the experimental data. It may be seen that the plume gets symmetric with increasing distance from the source. A small unevenness in Figure 4.7 at 2 " $(0.051 \mathrm{~m})$ below the centerline of the plume and $20 "(0.51 \mathrm{~m})$ away from the stack is not due to the lack of grid points, but it may be attributed to high amount of turbulence near the truck walls. There were 44 grid points used to plot the curves in Figure 4.7, which spanned from -30 " $(-0.76 \mathrm{~m})$ to +30 " $(0.76 \mathrm{~m})$ perpendicular to the centerline of the plume.

The dilution ratio (DR, defined as the reciprocal of $R_{c}$ (Equation 4.1) predicted by the CFD model is compared with the experimentally measured values along the centerline of the plume in Figure 4.8. It may be seen that at $55 \mathrm{mph}(88.51 \mathrm{~km} / \mathrm{hr})$ wind speeds, the dilution ratio increases rapidly to a value of 100 within 100 inches $(2.54 \mathrm{~m})$ downstream of the exhaust stack outlet. This is due to the fact that the high flow rate of ambient $126,358 \mathrm{ft}^{3} / \mathrm{s}\left(3,578 \mathrm{~m}^{3} / \mathrm{s}\right)$ is diluting the small flow rate of $13.4 \mathrm{ft}^{3} / \mathrm{s}\left(0.38 \mathrm{~m}^{3} / \mathrm{s}\right)$ coming out of the exhaust pipe. The small spikes on the centerline, as predicted by the CFD model, are due to the high amount of turbulence present near the exhaust pipe. The increase in dilution ratio on the centerline of the plume can be represented by the power law of the form $\left(\mathrm{DR}=\mathrm{Ax} \mathrm{x}^{\mathrm{n}}\right)$, where $\mathrm{x}$ is the distance $(\mathrm{m})$ from the stack. The power law predicted from the CFD simulations is $\mathrm{DR}=16.1 \times{ }^{1.36}$ matches well with the power law of the form $\mathrm{DR}=17.6 \times{ }^{1.3}$ from the experimental data. The CFD simulations clearly showed an increase in the dilution ratio with the increasing distance. 
The temperature variation predicted by the CFD model is compared with the experimentally measured values along the centerline of the plume in Figure 4.9. It may be seen that as the exhaust dilution increases, the temperature decreases rapidly to the ambient value of $75{ }^{\circ} \mathrm{F}\left(24{ }^{\circ} \mathrm{C}\right)$ within 100 inches $(2.54 \mathrm{~m})$ downstream of the exhaust stack outlet. This is explained by the discussion that pertained to Figures 4.6 and 4.8. That is, high flow rate of ambient air was diluting the small flow rate coming out of the exhaust pipe. Decrease in the temperature on the centerline of the plume can be represented by the power law of the form $\left(\mathrm{T}\left({ }^{\circ} \mathrm{C}\right)=\mathrm{Ax} \mathrm{x}^{\mathrm{n}}\right)$, where $\mathrm{x}$ is the distance $(\mathrm{m})$ from the stack. The power law predicted from the CFD simulations is $\mathrm{T}\left({ }^{\circ} \mathrm{C}\right)=34.5 \mathrm{x}^{-0.2}$ matches very well with the power law of the form $\mathrm{T}\left({ }^{\circ} \mathrm{C}\right)=35.3 \mathrm{x}^{-0.2}$ from the experimental data.

\subsubsection{Effect of Moving Gantry}

In the Langley tunnel, a moving gantry, which housed the measurement devices, was positioned near the exhaust stack. To determine the effect of this device near the exhaust stack on the structure of plume, the geometry was re-discretized inside the tunnel as shown in Figure 4.10. It may be observed from Figure 4.11 that the $\mathrm{CO}_{2}$ concentration variation in the lower part of the plume ( $\mathrm{z} \sim-10-20$ ”) from CFD simulation with the gantry agrees well with experimental data at 20 inches from the exhaust stack better than CFD simulation without the gantry (Figure 4.7). Since most of $\mathrm{CO}_{2}$ concentration diffuses quickly to the atmosphere within 35 inches, there is no significant difference at 40,80 , and 120 inches away from the exhaust stack. 


\subsubsection{Effect of Tractor-Trailer}

Trucks on the highways are traditionally accompanied by the trailers at their back. Because of the limited space of the tunnel, experimental data collected to validate the current study was taken on a truck without the trailer inside the Langley tunnel. However, it is worth analyzing the effect of the trailer on the structure of plume. To this end, a truck with the trailer, created using FLUENT software, is presented in Figure 4.12. The velocity and the temperature boundary conditions for this configuration are taken to be same as in Figure 4.4. The contours of relative concentration of $\mathrm{CO}_{2}$ coming out of a stack of the truck with the trailer at the back are presented on a plane passing through the center of the stack outlet in Figure 4.13. The CFD simulations clearly predict a longer plume for the trailer configuration compared to the truck without the trailer shown in Figure 4.4. The relative concentration of $\mathrm{CO}_{2}$ with the trailer decayed to the ambient concentration of 0.01 within $160 "(4.06 \mathrm{~m})$ from the stack, and the relative concentration decreased to 0.01 within 100 " $(2.54 \mathrm{~m})$ from the stack for the truck without the trailer. The plume remained attached to the body of the trailer, and hence it mixed slowly with the ambient as compared to the truck configuration without the trailer. Consequently, the plume decayed slowly in the presence of the trailer walls. 


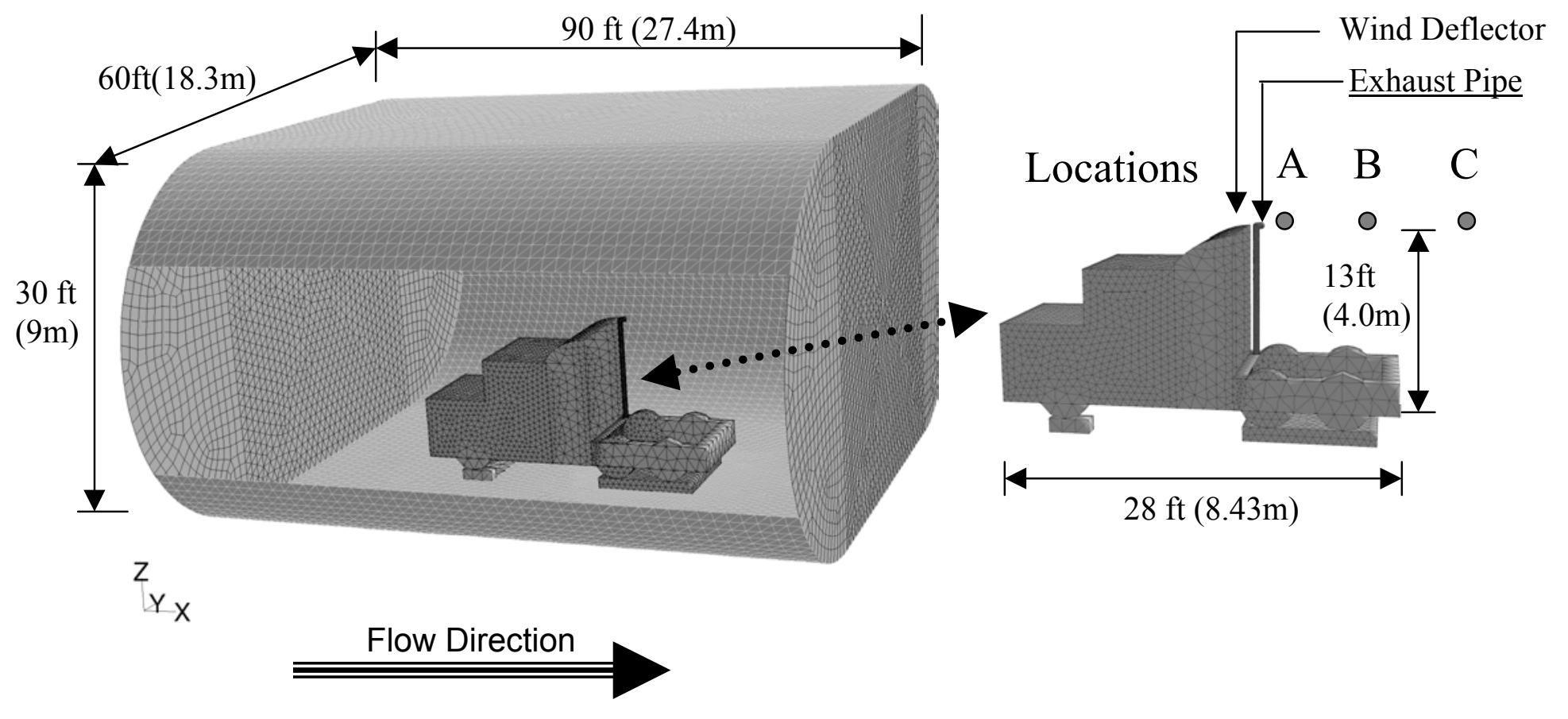

$\mathrm{D}$

○

Figure 4.1 Schematic grid of truck inside the wind tunnel

(Locations A, B, C and D are 20" (0.5 m), 80"(2.0 m), 200"(5.0m), and 337" (8.6 m)

respectively from the source) 


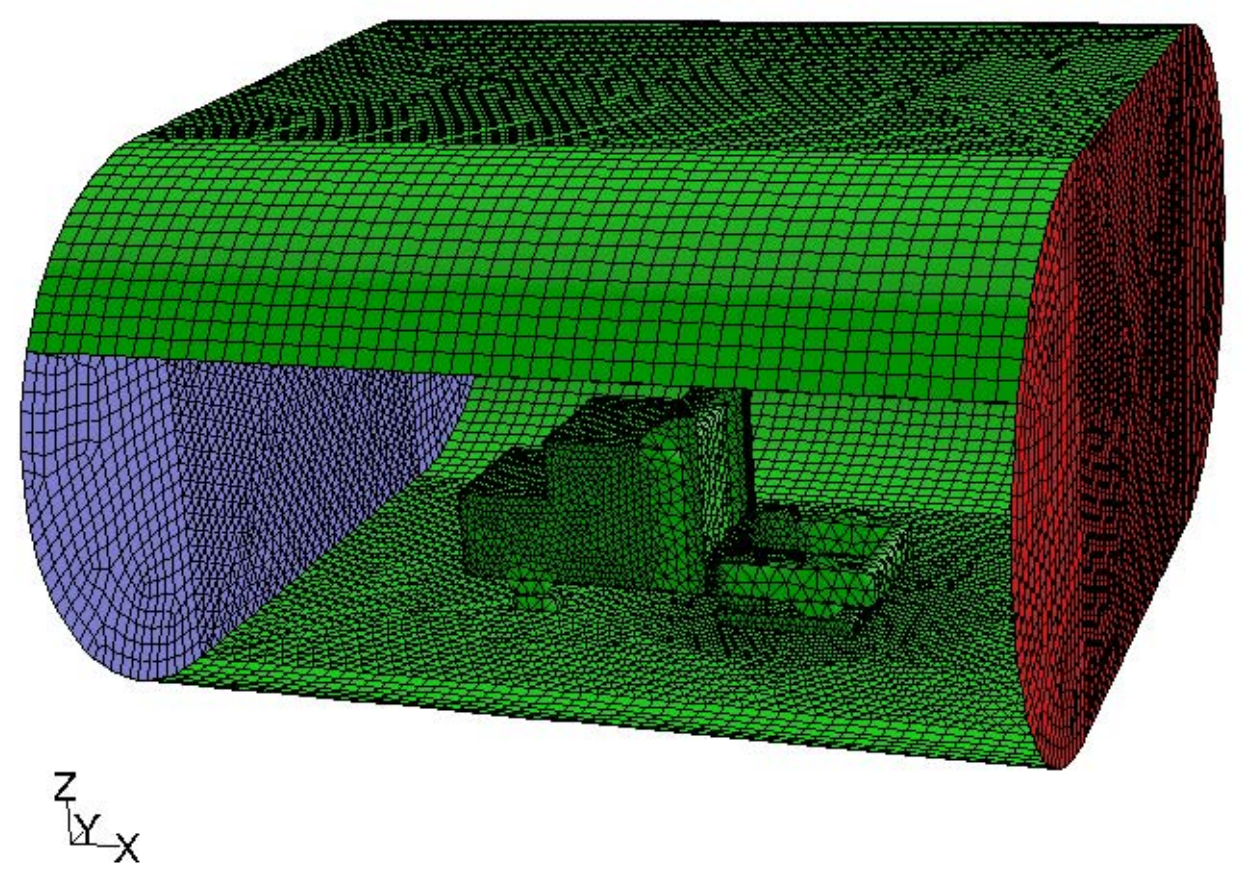

Figure 4.2 Computational grid of the truck inside the wind tunnel 


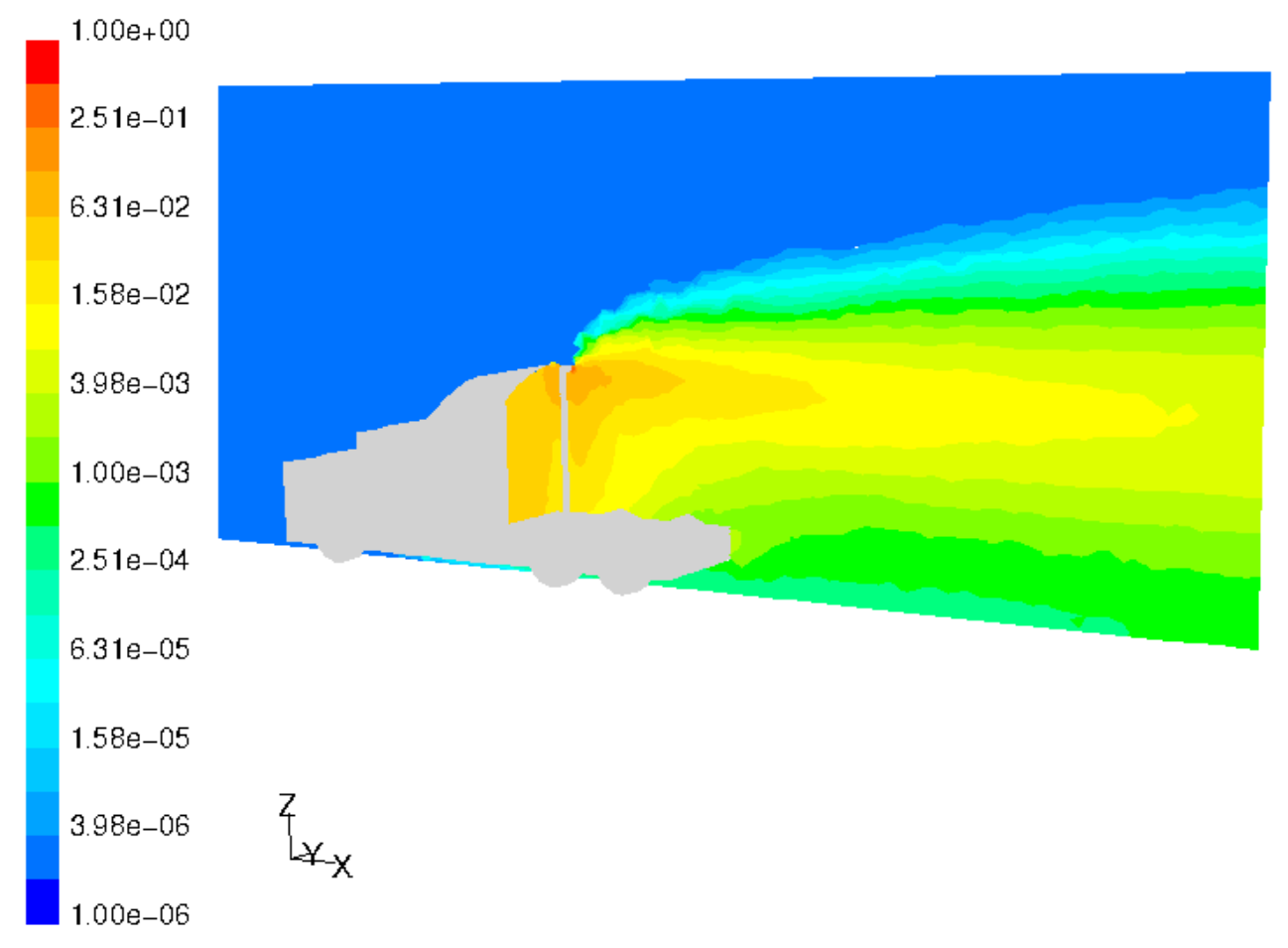

Figure 4.3 Filled contours of relative concentration of $\mathrm{CO}_{2}$ inside the wind tunnel 


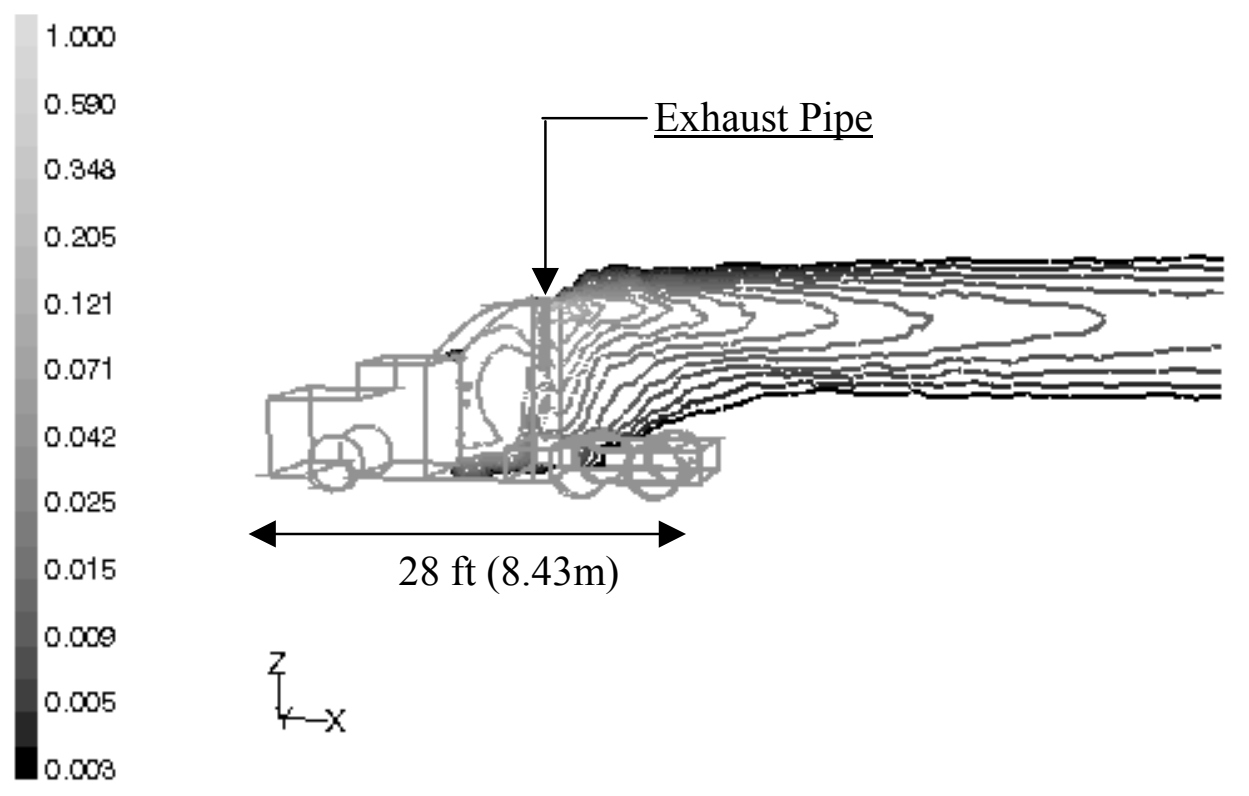

Figure 4.4 Contours of relative concentration (dimensionless) of $\mathrm{CO}_{2}$ inside the tunnel on a plane passing through the exhaust pipe 


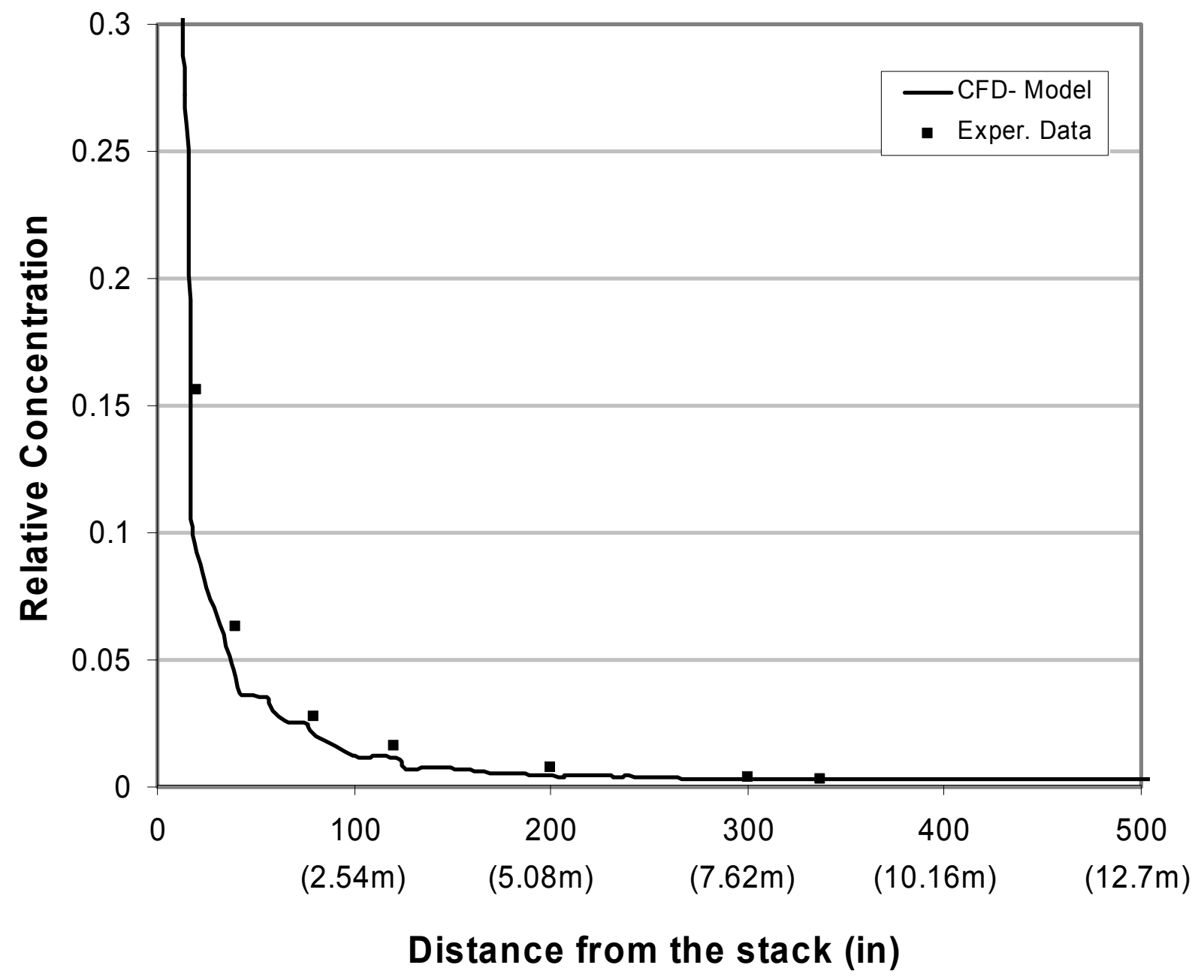

Figure 4.5 Variation of relative concentration of $\mathrm{CO}_{2}$ along the centerline of plume 


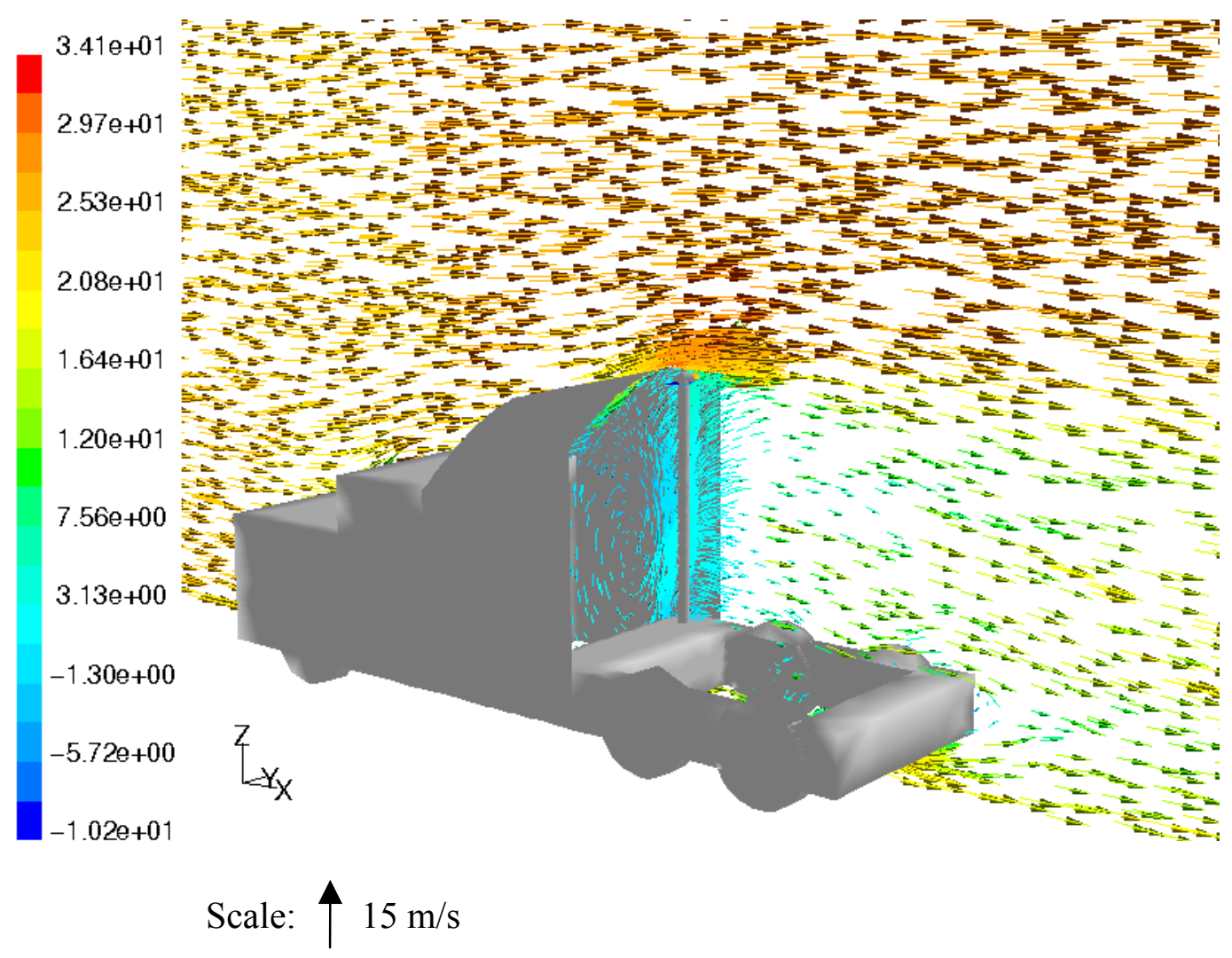

Figure 4.6 Velocity vectors $(\mathrm{m} / \mathrm{s})$ showing recirculation near the exhaust pipe of the truck 


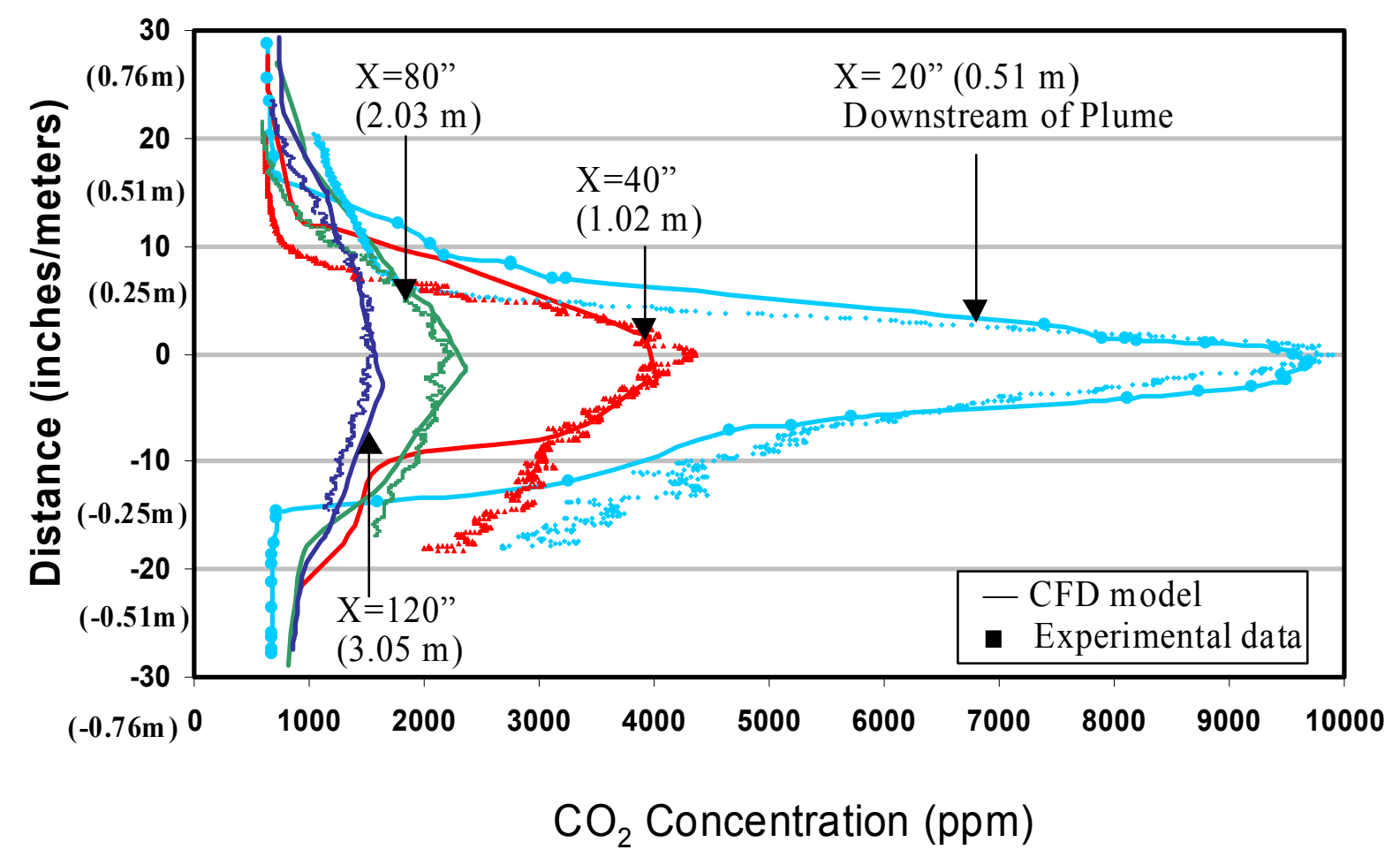

Figure 4.7 Variation of $\mathrm{CO}_{2}$ concentration inside the plume perpendicular to the centerline 


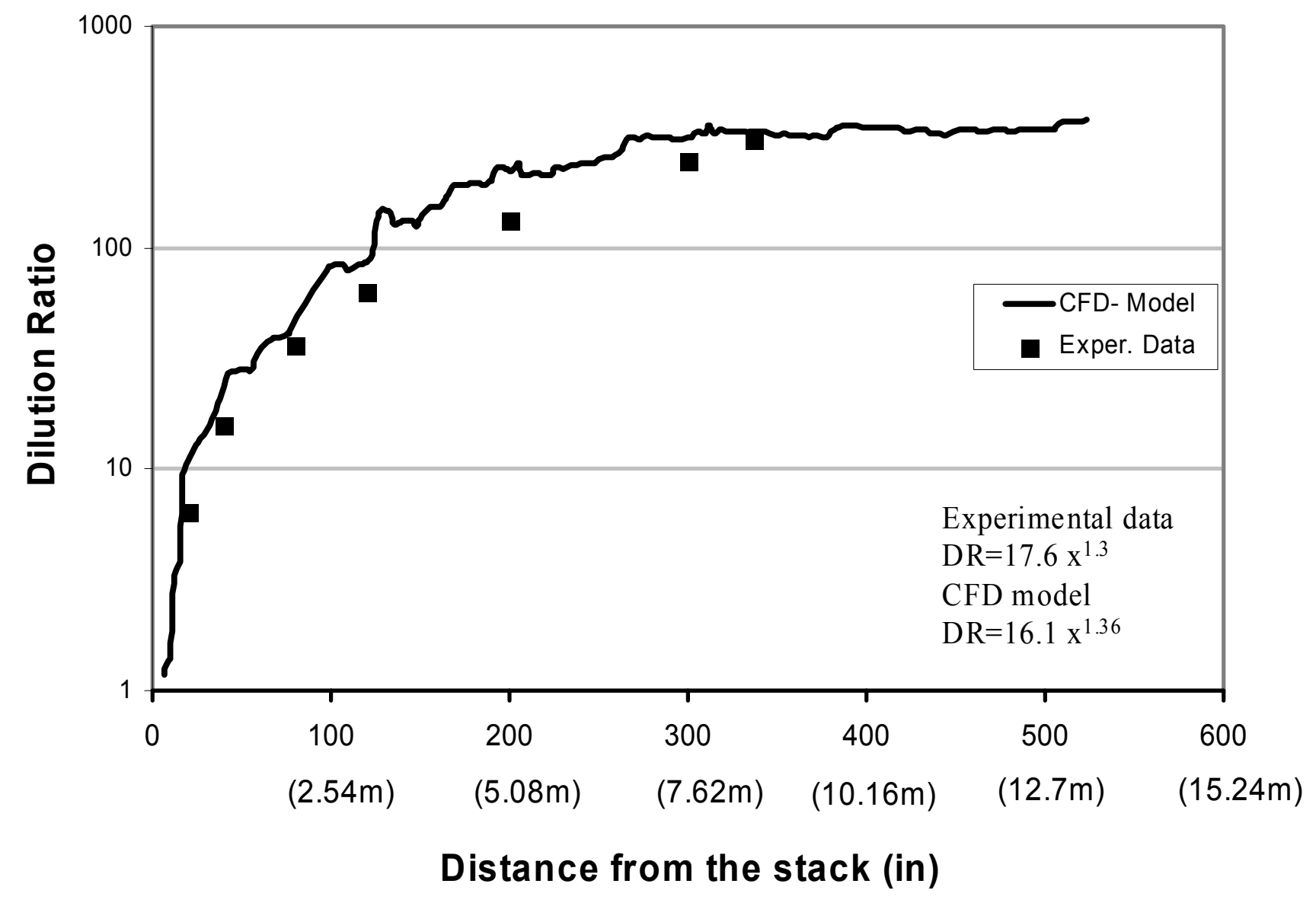

Figure 4.8 Variation of dilution ratio of $\mathrm{CO}_{2}$ along the centerline of plume 


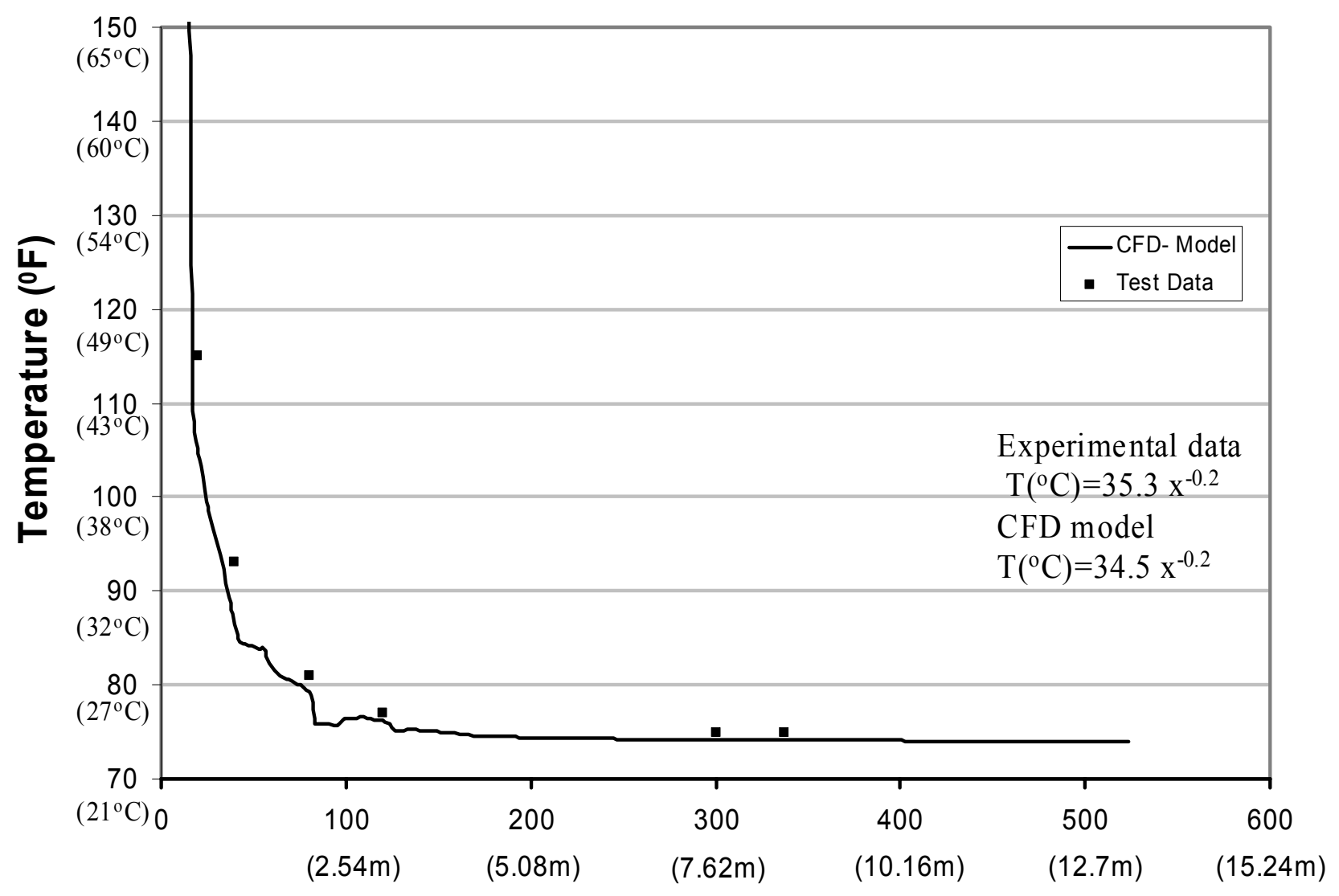

Distance from the stack (in)

Figure 4.9 Variation of temperature along the centerline of plume 


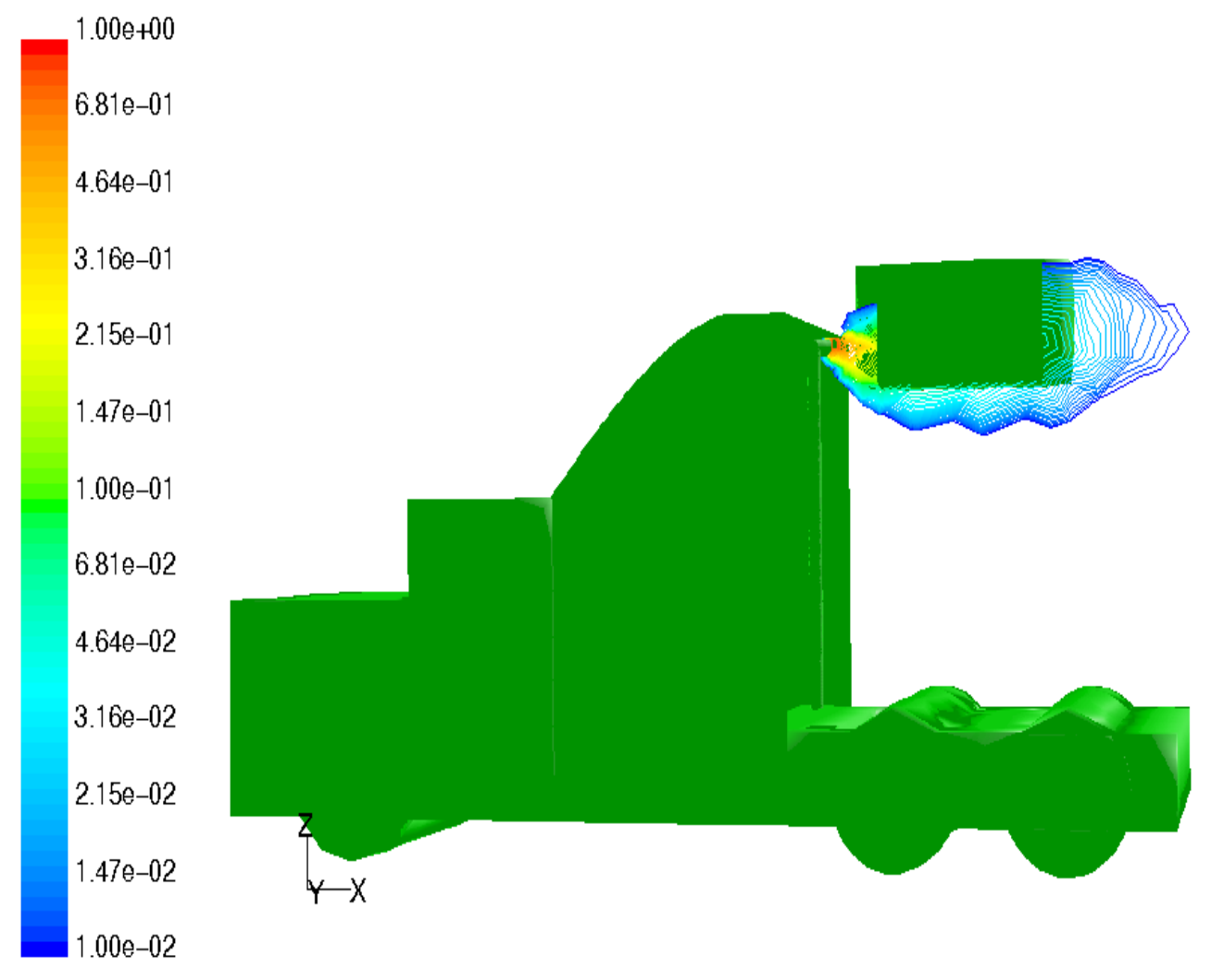

Figure 4.10 Relative concentration of $\mathrm{CO}_{2}$ near the moving gantry inside wind tunnel 


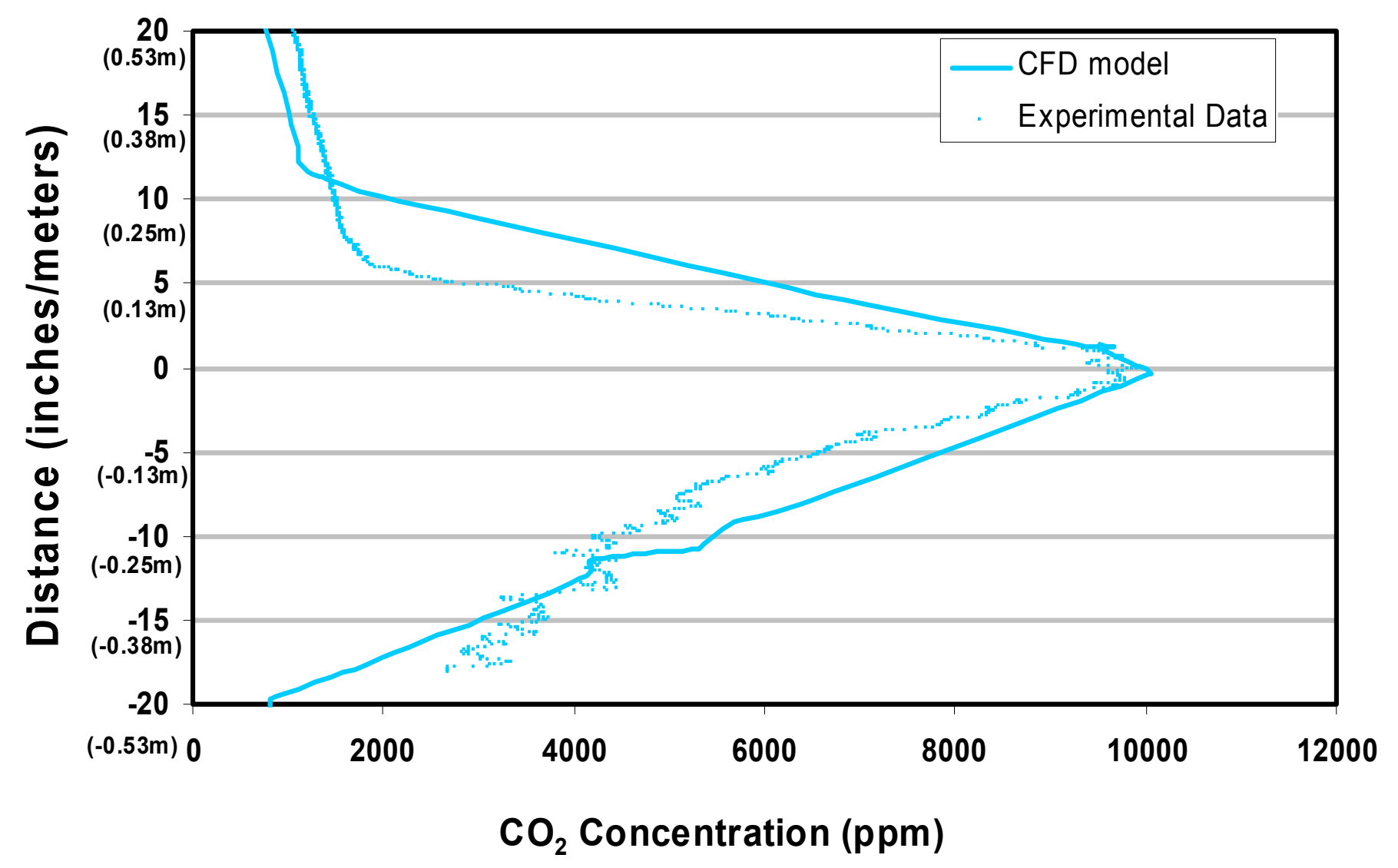

Figure 4.11 Variation of $\mathrm{CO}_{2}$ concentration inside the plume perpendicular to the ceterline near the moving gantry 


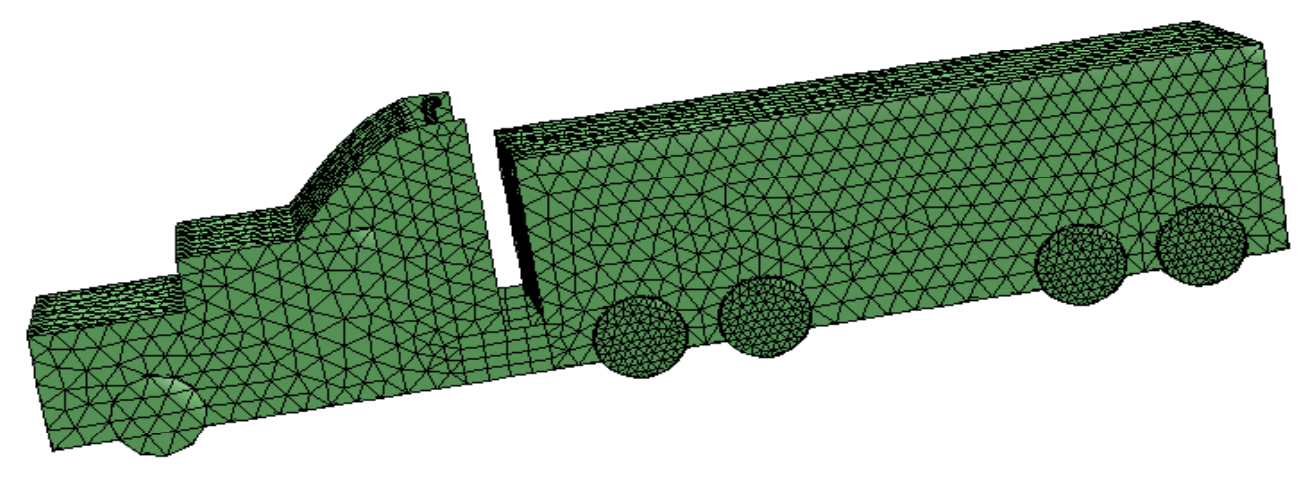

$\begin{aligned} & Z \\ & Y \\ & Y\end{aligned}-x$

Figure 4.12 Computational grid of tractor-trailer 


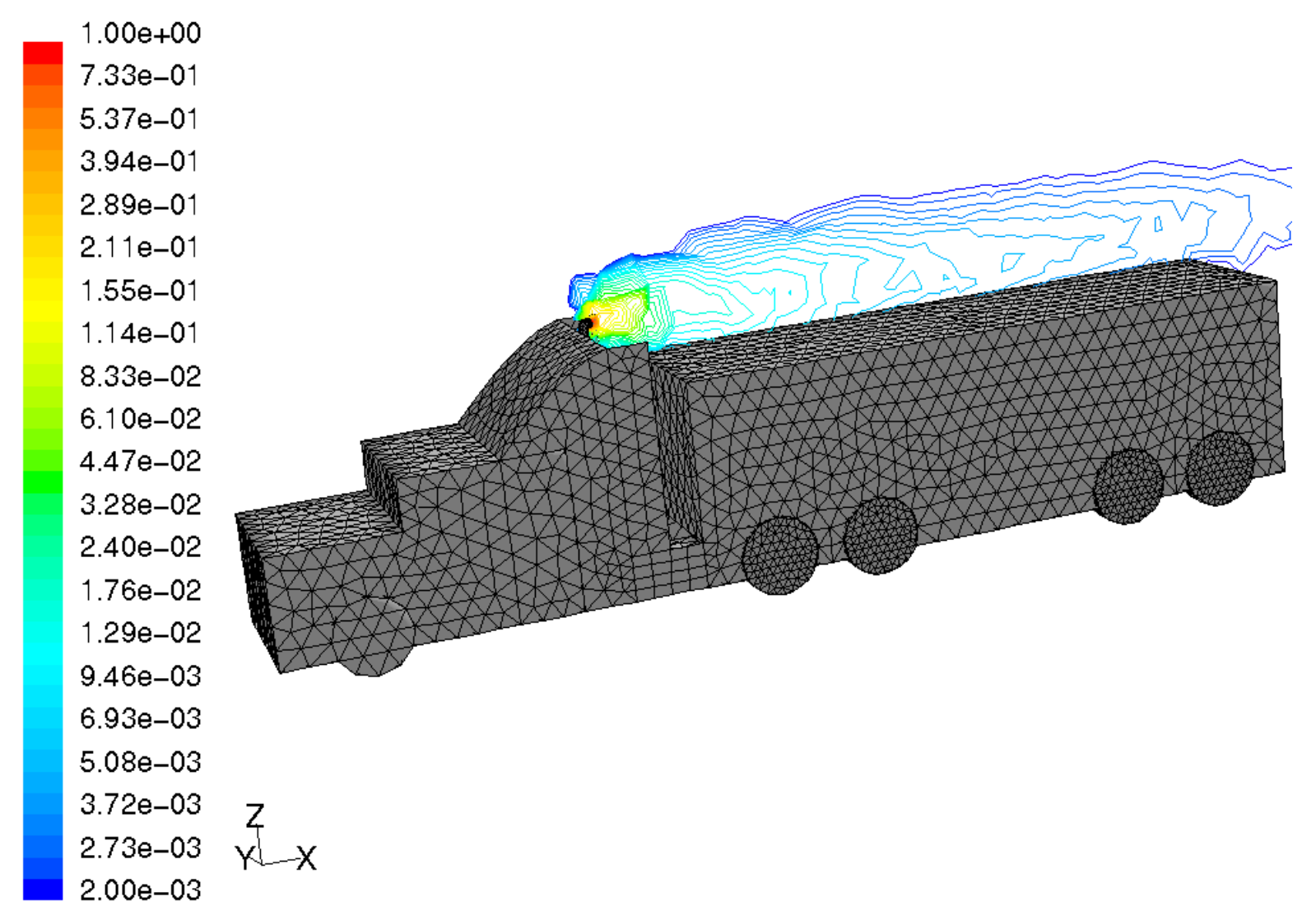

Figure 4.13 Contours of relative concentration (dimensionless) of $\mathrm{CO}_{2}$ on tractor-trailer 


\subsubsection{SIMULATION RESULTS USING GAUSSIAN AND PDF MODELS}

The $\mathrm{CO}_{2}$ concentration inside the dispersion plume predicted by the probability density function (PDF) mixture formulation from Equations (3.16) - (3.18) is compared with the experimentally measured values at a few locations downstream of the exhaust pipe in Figure 4.14. This formulation is different from the finite rate chemistry formulation presented in section 3.1.2. The predicted and measured $\mathrm{CO}_{2}$ values on a plume centerline are plotted at 20" $(0.51 \mathrm{~m}), 40 ”(1.02 \mathrm{~m}), 80 "(2.03 \mathrm{~m})$ and $120 "(3.05$ m) downstream of the exhaust pipe. At each of these 4 locations, raw $\mathrm{CO}_{2}$ concentrations are plotted to show their variation along a vertical line that is perpendicular to the centerline of the plume. It may be seen that the $\mathrm{CO}_{2}$ concentration decays close to the ambient $\mathrm{CO}_{2}$ concentration $(640 \mathrm{ppm})$ within a vertical distance of approximately \pm 30 " $(0.76 \mathrm{~m})$ from the centerline of the plume. Also, a slight asymmetry of the various curves may be observed about the abscissa (centerline) of the plume. This is due to the fact that plume disperses very quickly in the absence of any physical obstacle above the centerline. However, in the presence of physical obstacles the experimental plume decays much more slowly below the centerline. The re-circulation of air flow is also enhanced below the top arch wall (see Figure 4.1 for location of top arch) due to an opening below the back cabin which brings the under carriage air. It was noted from an additional exploratory simulation in the absence of any physical obstacles near the plume source, and without any undercarriage flow, the $\mathrm{CO}_{2}$ concentration was approximately 5-8\% higher than the current simulation at $20 "(0.508 \mathrm{~m})$ downstream of the source. The difference in $\mathrm{CO}_{2}$ concentration from above two cases is not significant at a distance away from the plume source. It is evident from Figure 4.14 that the $\mathrm{CO}_{2}$ concentration 
predicted by the CFD model agrees well with the experimental data. It may be seen that the plume becomes symmetric away from the source. . A small unevenness in Figure 4.14 at 2 " $(0.051 \mathrm{~m})$ below the centerline of the plume and 20 " $(0.51 \mathrm{~m})$ away from the stack is not due to the lack of grid points, but it may be attributed to high amount of turbulence near the truck walls. There were 44 grid points used to plot the curves in Figure 4.7, which spanned from -30 " $(-0.76 \mathrm{~m})$ to +30 " $(0.76 \mathrm{~m})$ perpendicular to the centerline of the plume.

The $\mathrm{CO}_{2}$ concentration inside the dispersion plume predicted by the modified Gaussian model (Equations 3.14 and 3.15) is also compared with the experimentally measured values at $20 "(0.51 \mathrm{~m}), 40 "(1.02 \mathrm{~m}), 80 "(2.03 \mathrm{~m})$ and $120 "(3.05 \mathrm{~m})$ downstream of the exhaust pipe. It is evident from Figure 4.15 that the $\mathrm{CO}_{2}$ concentration predicted by the modified Gaussian model agrees well with the experimental data, including values in the recirculation region. It is worth mentioning that once the Gaussian model is calibrated for a certain configuration then it may be used to give a fast accurate solution for different flow rates. 


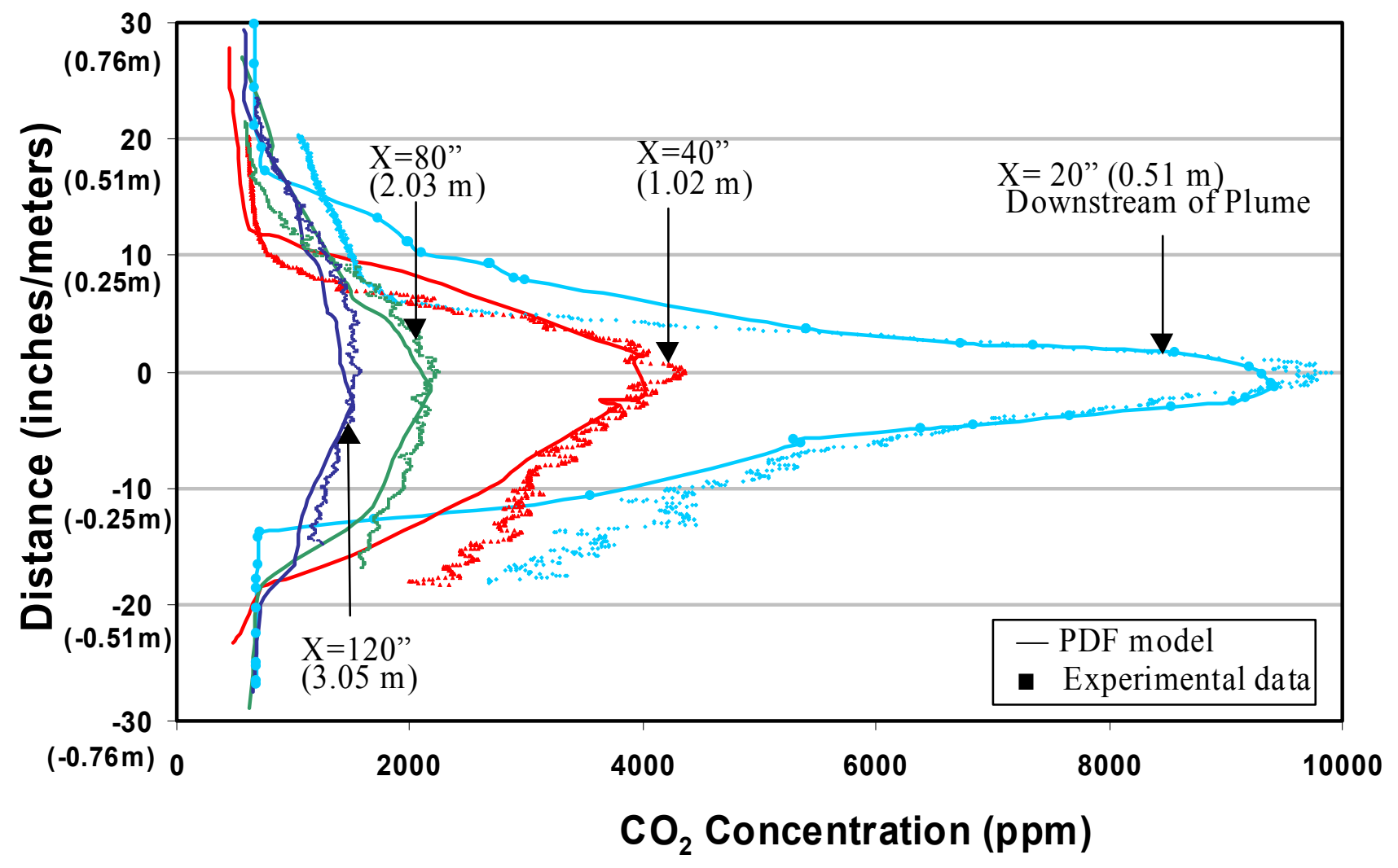

Figure 4.14 Variation of $\mathrm{CO}_{2}$ concentration inside the plume perpendicular to the centerline 


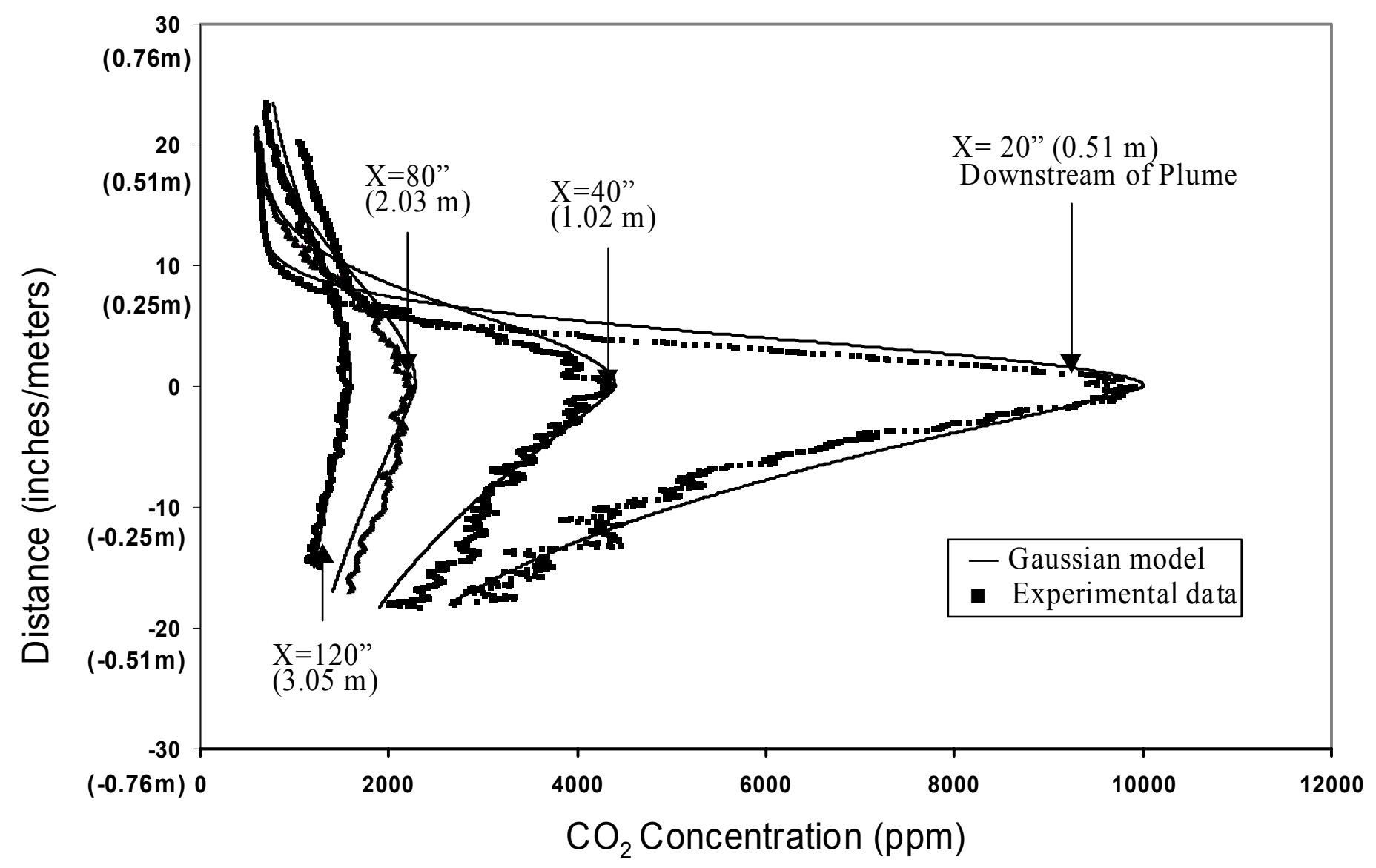

Figure 4.15 Variation of $\mathrm{CO}_{2}$ concentration inside the plume perpendicular to the centerline 


\subsection{EFFECT OF NUCLEATION ON PM FORMATION}

In order to determine the nucleation rate, it is necessary to convert the fuel sulfur to the $\mathrm{H}_{2} \mathrm{SO}_{4}$ vapor pressure in a dilution tunnel as done previously by Baumgard and Johnson (1996). First, the exhaust $\mathrm{SO}_{4}$ concentration can be determined from the following relation:

$\left[\mathrm{SO}_{4}\right]=(\mathrm{F} / \mathrm{A}) *(\%$ fuel sulfur $) *\left(\% \mathrm{~S}\right.$ to $\mathrm{SO}_{4}$ conv. $) *\left(\mathrm{M}_{\mathrm{SO}_{4}} / \mathrm{M}_{\text {exhaust }}\right) *(1-\mathrm{F} / \mathrm{A}) *$ density $)(4.2)$

where $(\mathrm{F} / \mathrm{A}=0.035)$ is the fuel to air ratio, wt. \%fuel sulfur for this fuel is $50 \mathrm{ppm}, \mathrm{M}_{\mathrm{SO} 4}$ is the molecular weight of $\mathrm{SO}_{4}, \mathrm{M}_{\text {exhaust }}$ is the apparent molecular weight of the exhaust stream, $\% \mathrm{~S}$ to $\mathrm{SO}_{4}$ is assumed to be 0.04 (Baumgard and Johnson, 1996, Shi and Harrison, 1999), and the density of air is assumed to be $1.186 \mathrm{~kg} / \mathrm{m}^{3}$. The next step is to convert the actual dilution tunnel $\mathrm{SO}_{4}$ concentration to $\mathrm{H}_{2} \mathrm{SO}_{4}$ vapor pressure using the ideal gas law:

$$
\mathrm{P}_{\mathrm{H} 2 \mathrm{SO} 4}=(\mathrm{m} / \mathrm{V}) \mathrm{RT} / \mathrm{M}
$$

where $\mathrm{V}$ is the volume, $\mathrm{m}$ is the mass of $\mathrm{H}_{2} \mathrm{SO}_{4}, \mathrm{R}$ is the universe gas constant, $\mathrm{M}$ is the molecular weight of exhaust, and $\mathrm{T}$ is the temperature.

The effect of relative humidity on the critical nucleation particle diameter (stable nucleus diameter) and the nucleation rate as a function of time at $25^{\circ} \mathrm{C}$ is shown in Figure 4.16. It may be seen that the nucleus diameter decreases with the increasing relative humidity, and the nucleation rate increases with the increasing relative humidity. Similar 
trends were also observed by Shi and Harrison (1999). At the lower relative humidity, more molecules are necessary to obtain the critical energy required for the nucleus to be stable and grow, and consequently the particle diameter is larger. This occurs because, at the lower relative humidity, there will be a tendency for the particles to evaporate. Conversely, the higher the relative humidity, the smaller the particle diameter necessary to become a stable particle.

The nucleation rate as a function of $\mathrm{H}_{2} \mathrm{SO}_{4}$ vapor pressure at a constant relative humidity $(20 \%)$ and $25{ }^{\circ} \mathrm{C}$ and $46{ }^{\circ} \mathrm{C}$ is shown in Figure 4.17 . These findings are similar to Baumgard and Johnson (1996), and Shi and Harrison (1999). Increasing the temperature decreases the nucleation rate mainly because the $\Delta \mathrm{G}$ value increases almost an order of magnitude due to an increase in $\mathrm{H}_{2} \mathrm{SO}_{4}$ vapor pressure from $25{ }^{\circ} \mathrm{C}$ to $46{ }^{\circ} \mathrm{C}$. Because nucleation rate is proportional to $\exp (-\Delta \mathrm{G} / \mathrm{kT})$, the larger the $\Delta \mathrm{G}$ value, the lower the nucleation rate. This may be attributed to the fact that at higher temperature, the vapor molecules stay in the vapor-state instead of condensing onto each other.

The effect of relative humidity on critical nucleation particle diameter (stable diameter) at temperature $25{ }^{\circ} \mathrm{C}$ is shown in Figure 4.18. The higher the relative humidity, the smaller the particle diameter necessary to become a stable particle. At the lower relative humidity, it requires more energy to get over the saddle point and, therefore, more molecules are necessary to obtain this energy and consequently the particle diameter is large. 
The effect of relative humidity on nucleation rate at temperature $25^{\circ} \mathrm{C}$ is shown in Figure 4.19. The trend indicates that the higher relative humidity enhances nucleation. This can be explained by examining the frequency factor "C" ( Equation. 3.28). At the higher relative humidity, the bulk phase density of the water molecules also increases, leading to more molecules colliding pre unit time. Therefore, nucleation rate increases with the increasing relative humidity.

Figure 4.20 depicts the effect of relative humidity on sulfuric acid vapor pressure with time for $13.1 \mathrm{ppm} \mathrm{S}$ fuel. It may be seen that the sulfuric acid vapor pressure decreases with the increase in relative humidity. At $20 \%$ relative humidity, the value of sulfuric acid vapor pressure remained at about constant $4.5 \times 10^{-5} \mathrm{~mm} \mathrm{Hg}$. However, when the higher relative humidity (such as $35 \%$ or $45 \%$ ) was applied, the value of sulfuric acid vapor pressure dropped much faster than the case with the $20 \%$ relative humidity. This similar trend may be seen from the Figure 4.21 and Figure 4.22 with the fuel sulfur content of $125 \mathrm{ppm}$ and $300 \mathrm{ppm}$, respectively. This may be due to the fact that the decrease in partial vapor pressure for sulfuric acid is proportional to the nucleation rate at a given time. Therefore, decrease in the partial vapor pressure for sulfuric acid for $20 \%$ relative humidity case is the smallest compared to the $35 \%$ and $45 \%$ relative humidity cases.

Figure 4.23 shows the effect of relative humidity on diameter of a nucleated particle with time for the $13.1 \mathrm{ppm}$ sulfur fuel. It may be seen that the nucleus diameter 
decreases with the increasing relative humidity. At the lower relative humidity, more molecules are necessary to obtain the critical energy required for the nucleus to be stable and grow, and consequently the particle diameter is larger. This similar trend may be observed in Figures 4.24 and 4.25 with the fuel sulfur content of $125 \mathrm{ppm}$ and $300 \mathrm{ppm}$, respectively.

Figure 4.26 presents the effect of relative humidity on nucleation rate as a function of elapsed time in the $13.1 \mathrm{ppm}$ sulfur fuel content. It should be mentioned here that the nucleation rate increases with the increase in relative humidity. At the higher relative humidity, the bulk phase density of the water molecules also increases, leading to more molecules colliding per unit time. At the $20 \%$ relative humidity, the nucleation rate remained almost constant. It may be seen from Figure 4.26 that when the relative humidity increases, the nucleation rate decreases slightly with time. This trend can be also observed in Figure 4.27 and Figure 4.28 with the fuel sulfur content of $125 \mathrm{ppm}$ and 300 ppm, respectively.

The variation of nucleation rate with time for different sulfur fuel content at constant relative humidity of $20 \%$ is presented in Figure 4.29. As the percentage of sulfur fuel content increases, the nucleation rate increases. Higher the percentage of fuel sulfur content, gives higher $\mathrm{SO}_{4}$ concentration, which leads to higher nucleation rate. Figure 4.30 shows the variation of nucleation rate with time for fuels with different sulfur contents at constant relative humidity of $35 \%$. It may be seen from Figure 4.30 that the nucleation rate decreases slightly with time for fuel with sulfur content of $300 \mathrm{ppm}$ 
compared with sulfur content of $125 \mathrm{ppm}$ and $13.1 \mathrm{ppm}$. This trend can be also seen in Figure 4.31 for the relative humidity of $45 \%$. This trend is similar to the trends already discussed on the examples shown in Figures 4.29 and 4.30.

Figure 4.32 shows the temporal variation of nucleus diameter with time in fuels with different fuel sulfur content at a constant relative humidity $20 \%$. As the sulfur fuel content increases, the nucleus diameter also decreases. Figure 4.33 shows the variation of nucleus diameter with time in different sulfur fuel content at constant relative humidity $35 \%$. It may be seen from Figure 4.33 that the nucleus diameter increases more rapidly with time at sulfur fuel content $300 \mathrm{ppm}$ compared with the sulfur fuel content of 125 ppm or $13.1 \mathrm{ppm}$. It may be seen that the diameter increases with time at the sulfur fuel content $125 \mathrm{ppm}$ and $13.1 \mathrm{ppm}$ as shown in Figure 4.34 with relative humidity $45 \%$.

Figure 4.35 presents the temporal variation of sulfuric acid vapor pressure with time for different sulfur fuel contents at constant relative humidity $20 \%$. Higher sulfur fuel content gives the higher sulfuric acid vapor pressure. As the sulfur fuel content increases, consequently $\mathrm{SO}_{4}$ concentration increases. Figure 4.36 shows the variation of sulfuric acid vapor pressure with time for different sulfur fuel contents at constant relative humidity of $35 \%$. It may be seen that the sulfuric acid vapor pressure decrease more rapidly with time for sulfur fuel content $300 \mathrm{ppm}$ than for the fuels with the sulfur content of $125 \mathrm{ppm}$ or $13.1 \mathrm{ppm}$. This trend can also be observed in much higher relative humidity of (45\%) in Figure 4.37. This may be due to the fact that the decrease in partial vapor pressure for sulfuric acid is proportional to the nucleation rate at a given time. 
Therefore, the decrease in the partial vapor pressure for sulfuric acid for $13.1 \mathrm{ppm}$ case is smaller than for the $125 \mathrm{ppm}$ and 300 ppm cases. 


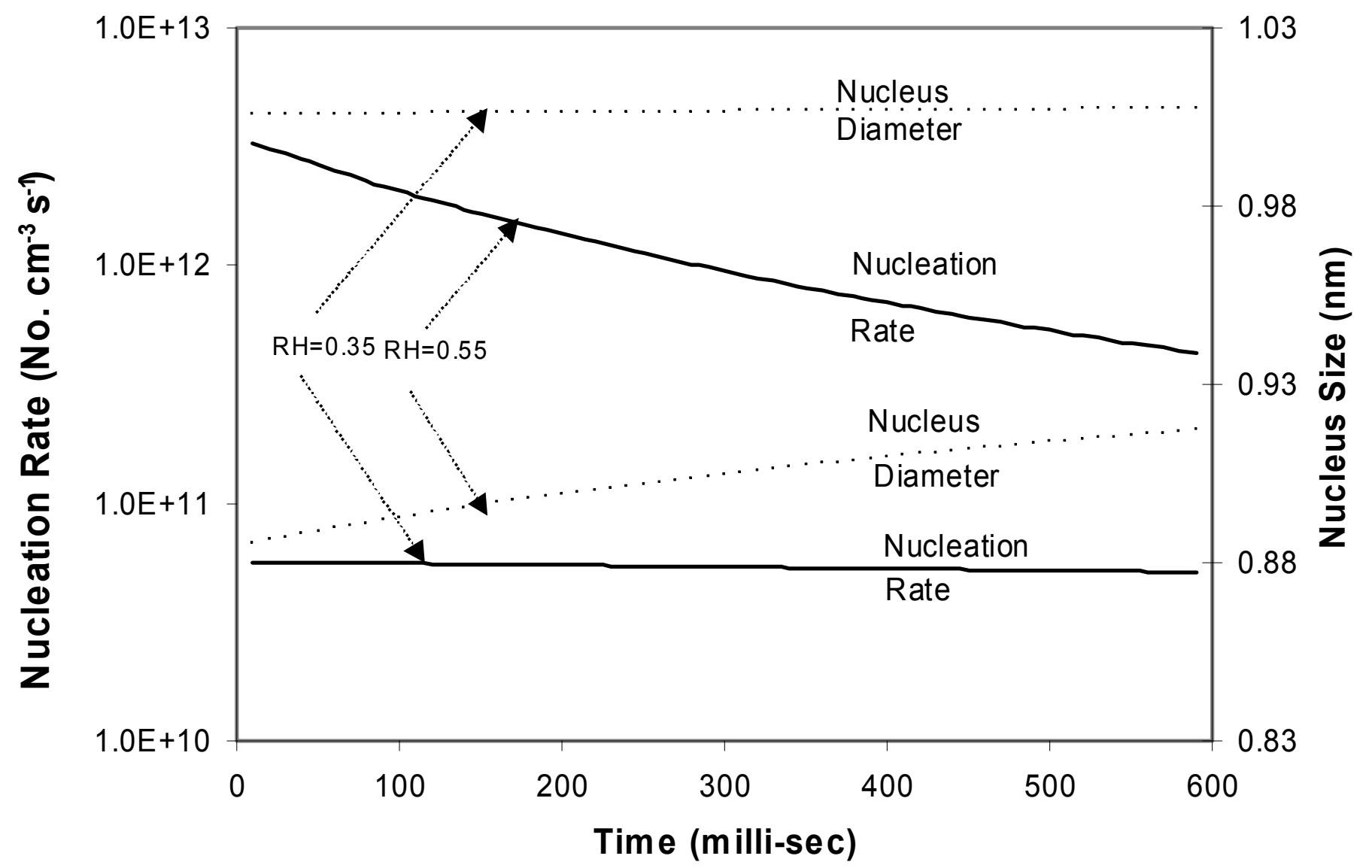

Figure 4.16 Effect of relative humidity on nucleation rate and nucleus size 


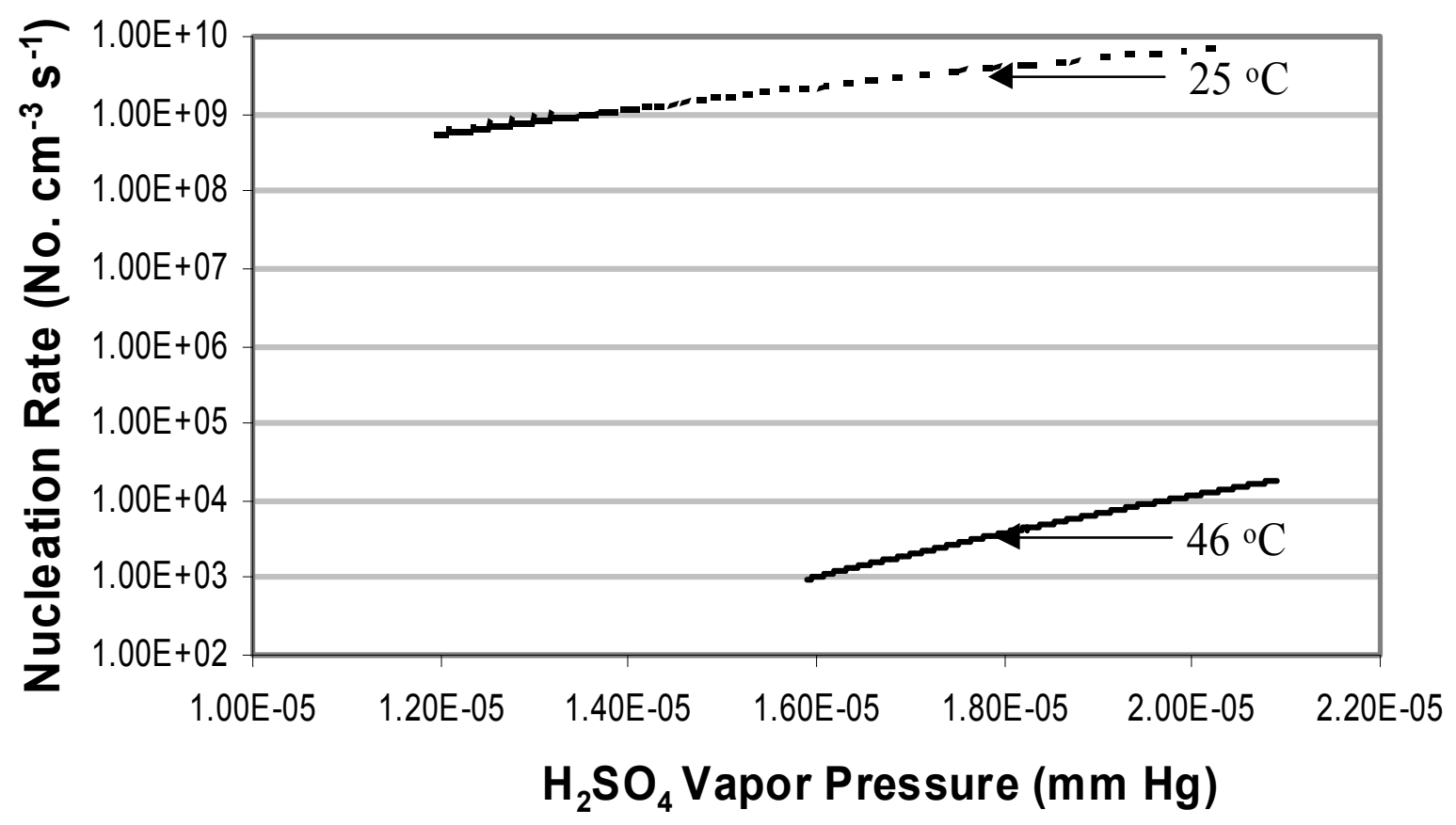

Figure 4.17 Nucleation rate as a function of dilution tunnel $\mathrm{H}_{2} \mathrm{SO}_{4}$ vapor pressure at a constant $20 \%$ relative humidity and temperature $25{ }^{\circ} \mathrm{C}$ and $46{ }^{\circ} \mathrm{C}$ 


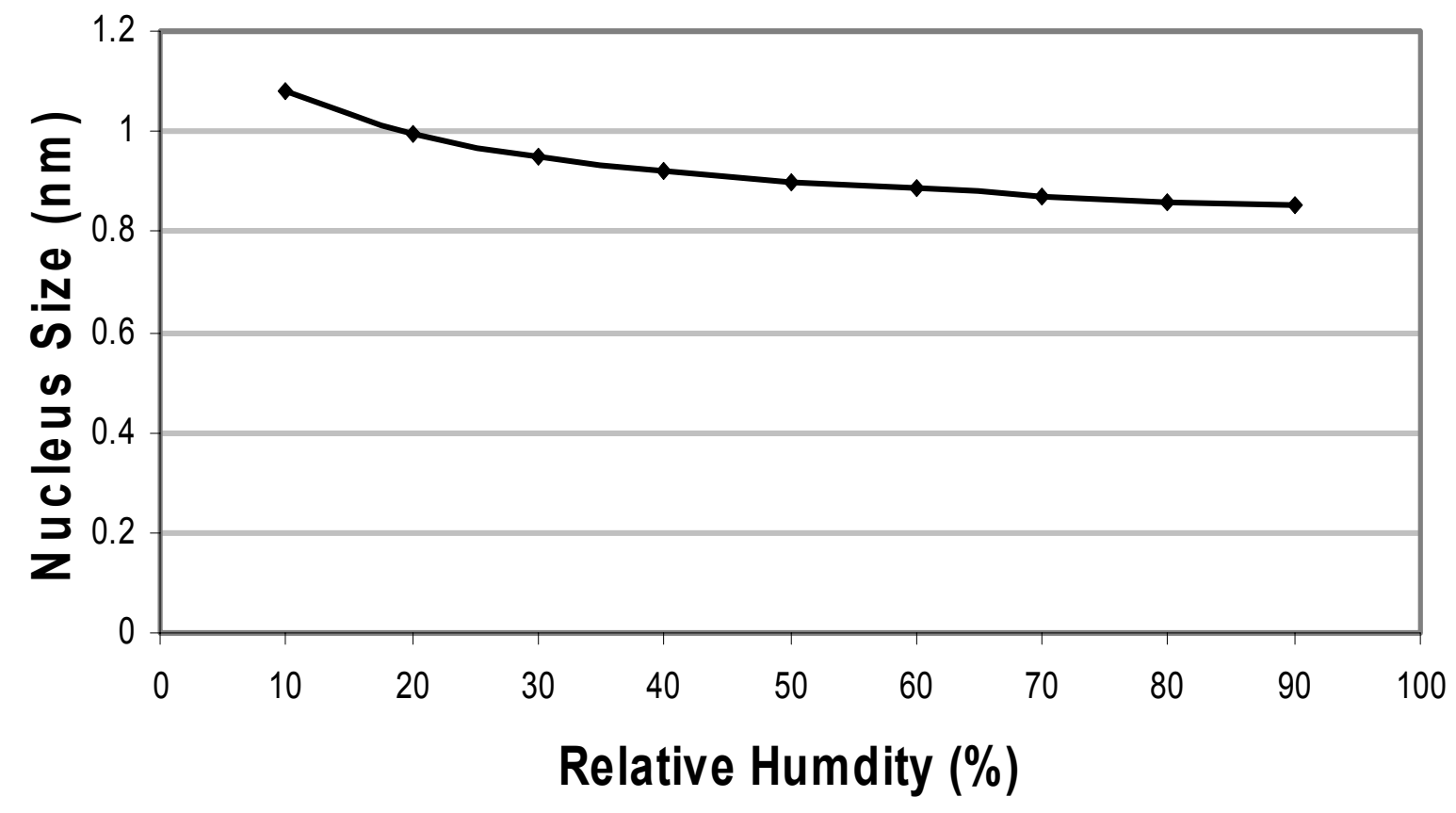

Figure 4.18 Effect of critical nucleation diameter as a function of relative humidity 


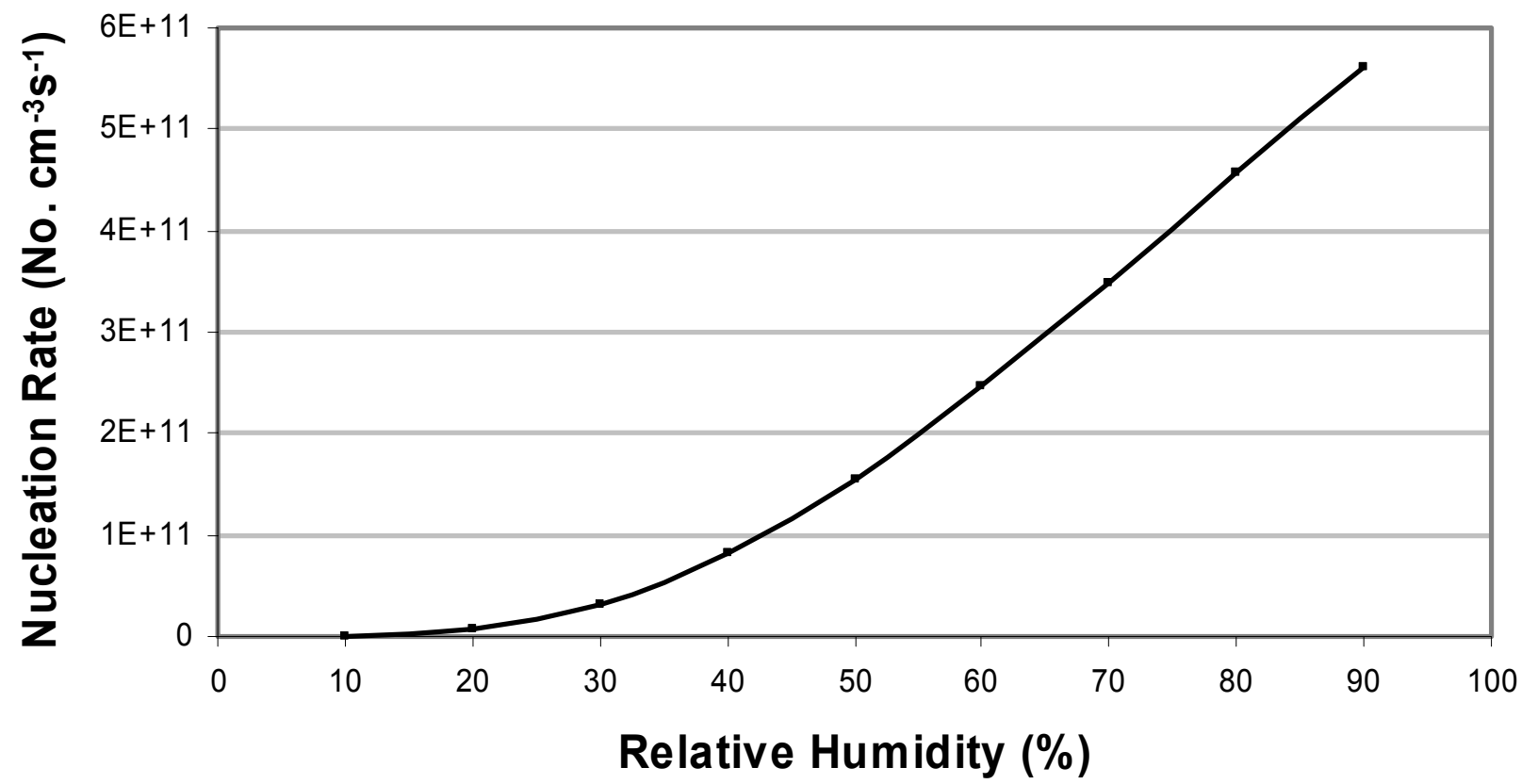

Figure 4.19 Effect of relative humidity on nucleation rate at $25^{\circ} \mathrm{C}$ 


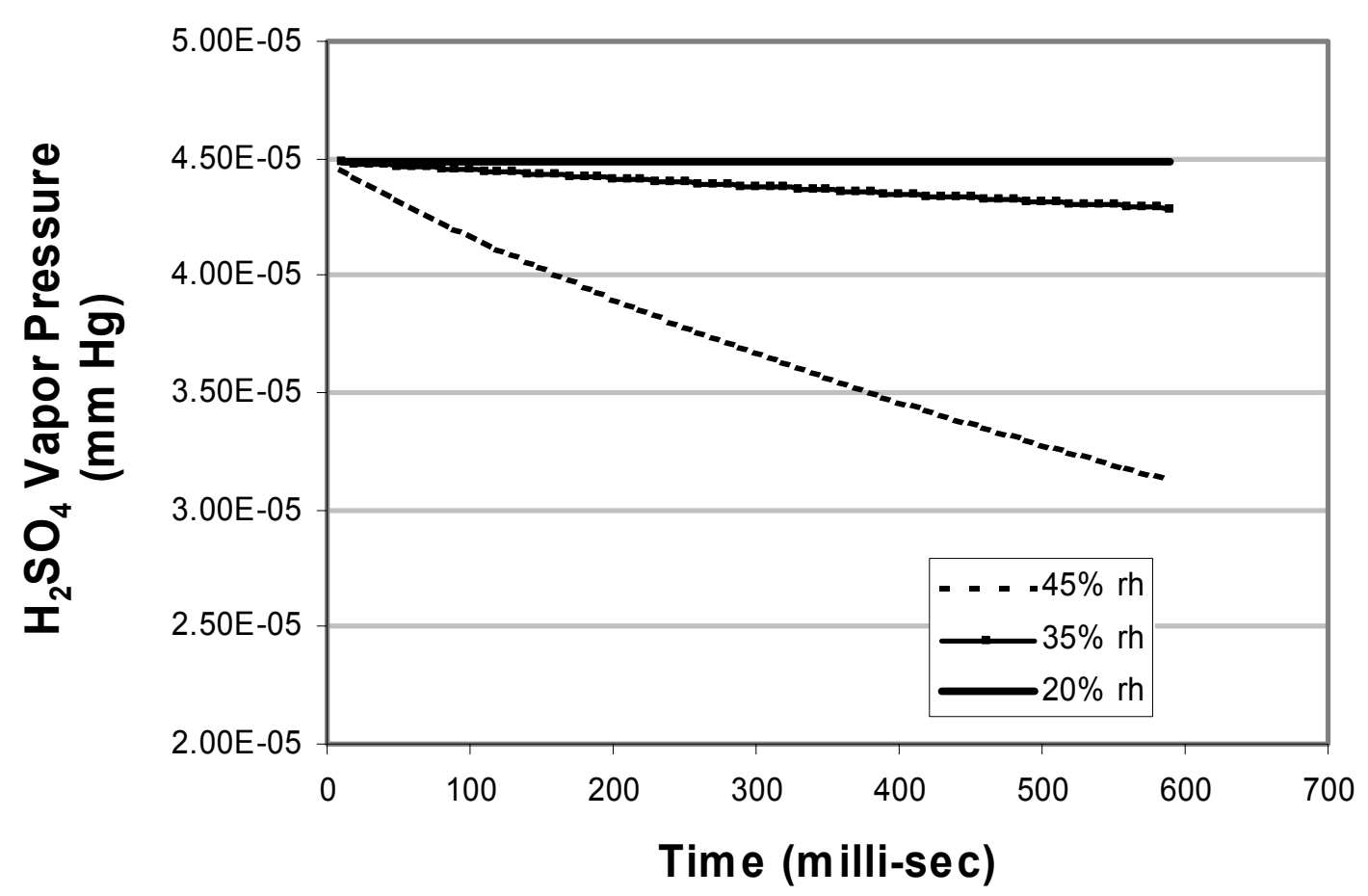

Figure 4.20 Effect of relative humidity on sulfuric acid vapor pressure with time for $13.1 \mathrm{ppm}$ sulfur fuel 


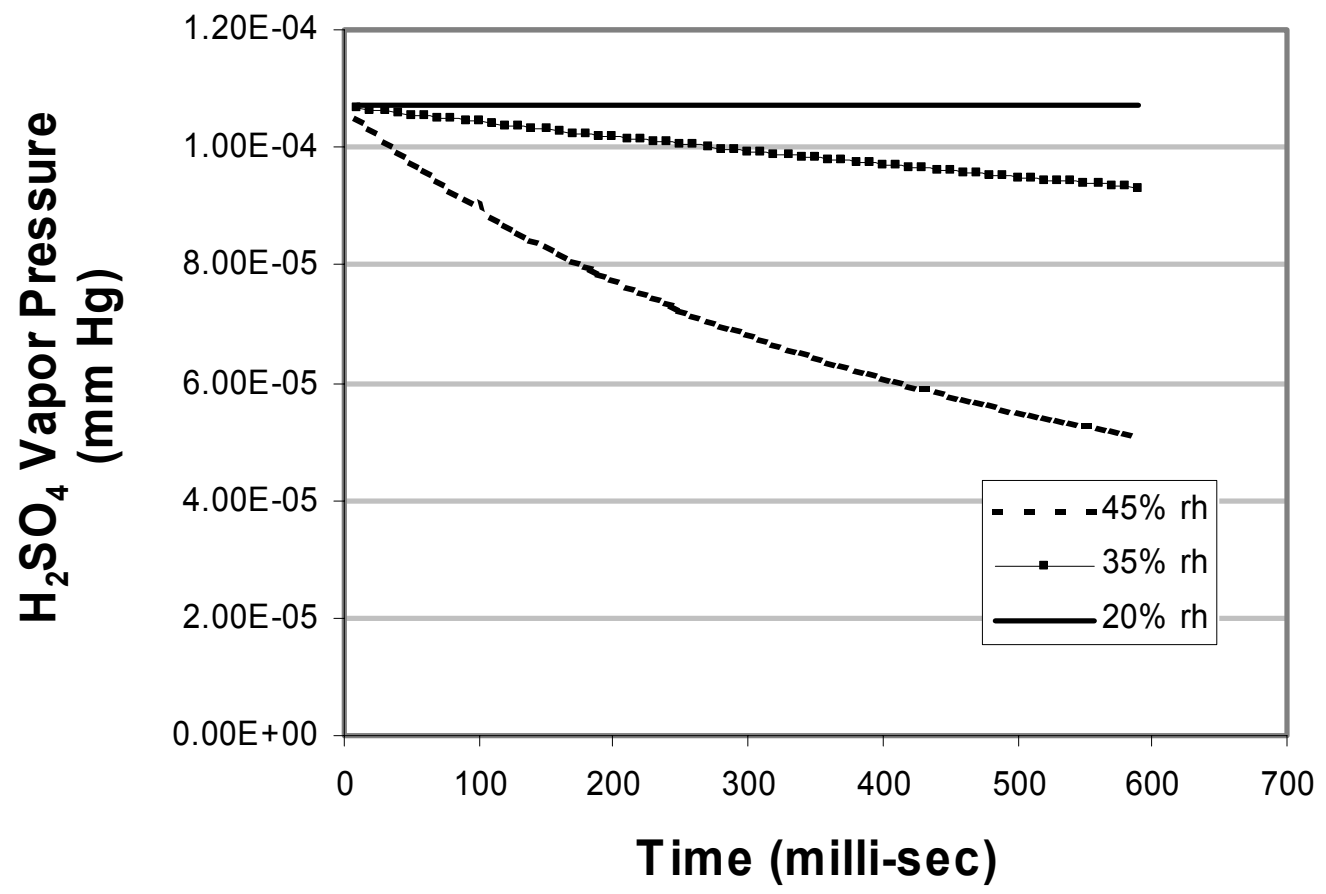

Figure 4.21 Effect of relative humidity on sulfuric acid vapor pressure with time for $125 \mathrm{ppm}$ sulfur fuel 


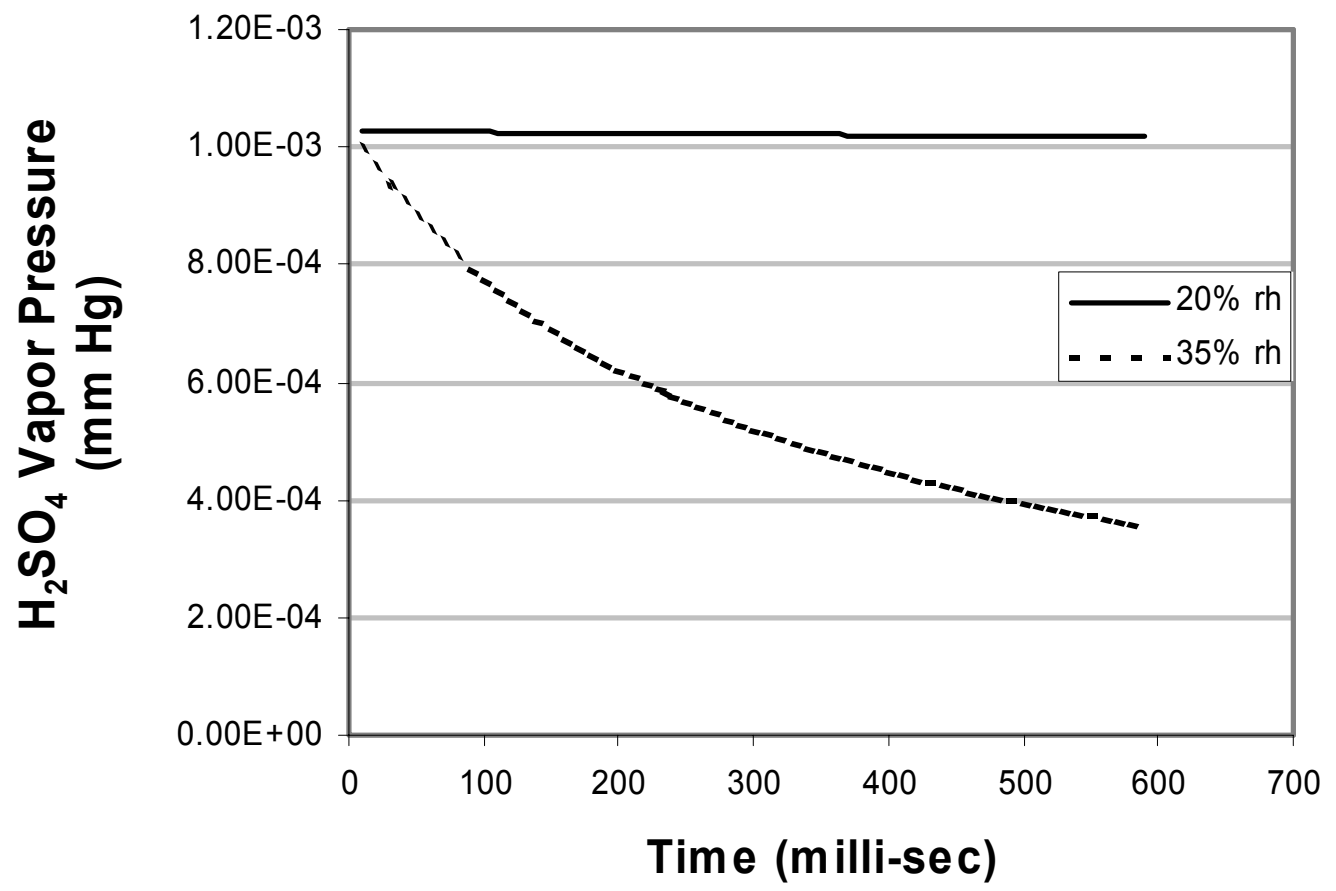

Figure 4.22 Effect of relative humidity on sulfuric acid vapor pressure with time for 300 ppm sulfur fuel 


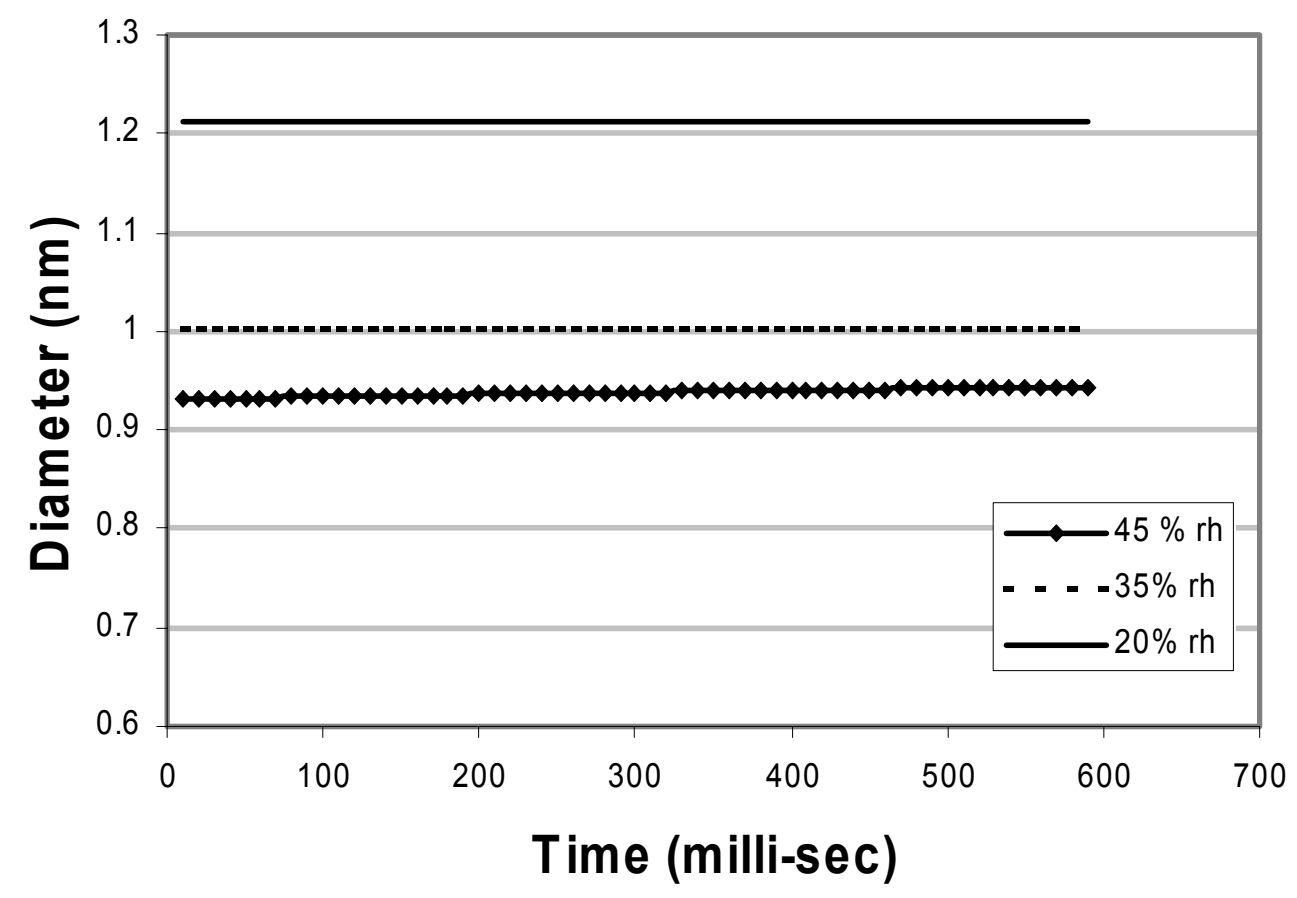

Figure 4.23 Effect of relative humidity on nucleus diameter for $13.1 \mathrm{ppm}$ sulfur fuel 


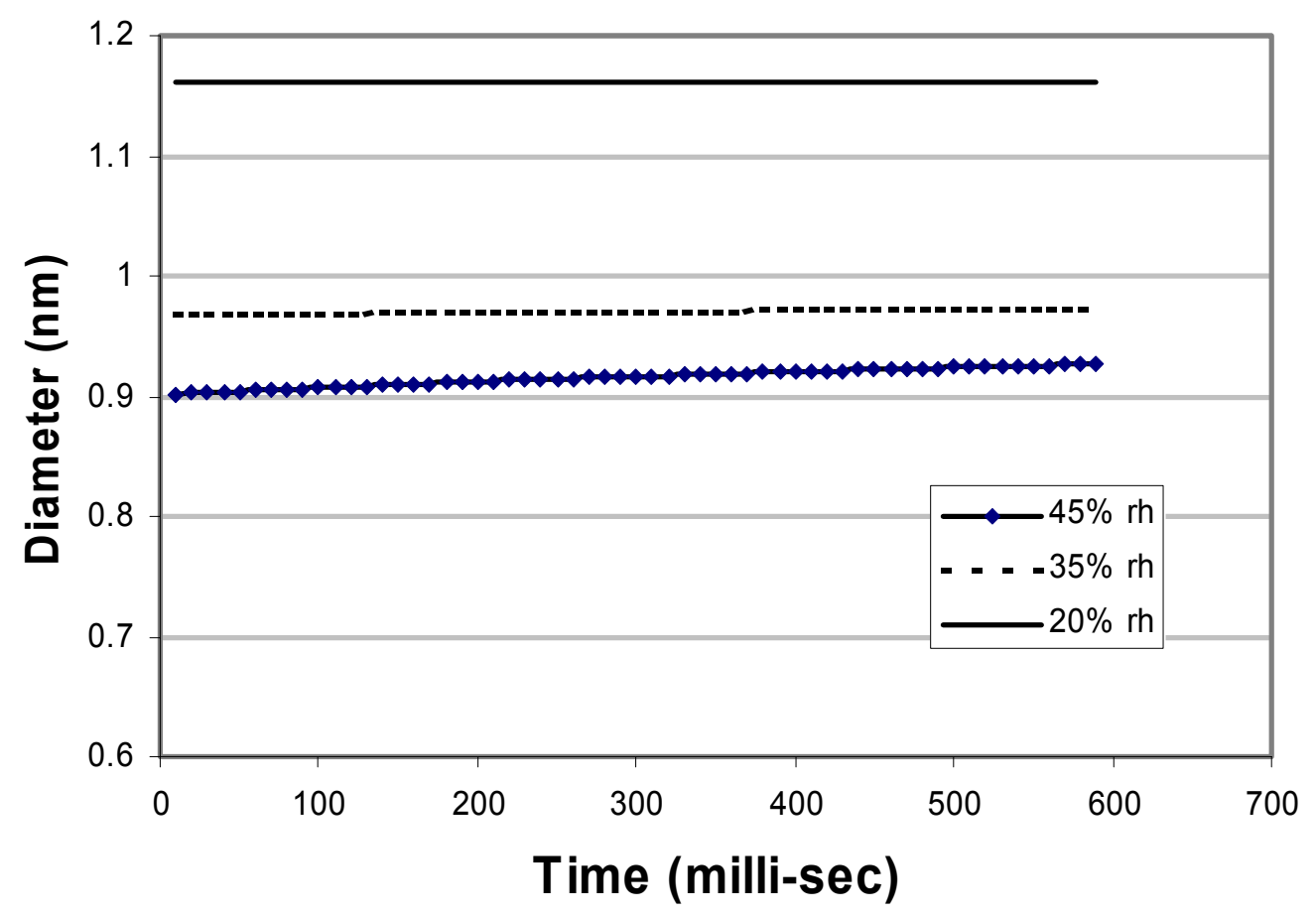

Figure 4.24 Effect of relative humidity on nucleus diameter for $125 \mathrm{ppm}$ sulfur fuel 


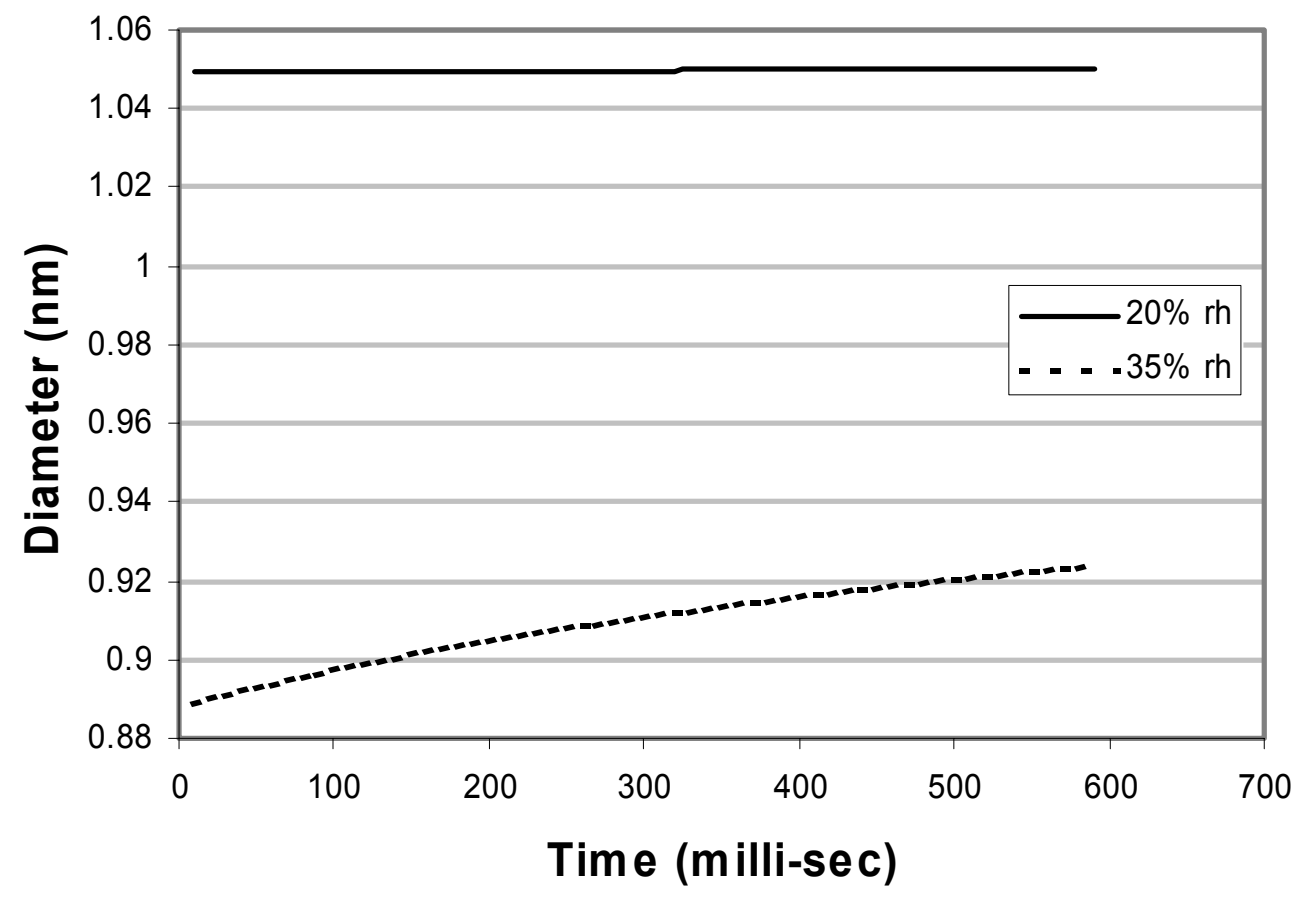

Figure 4.25 Effect of relative humidity on nucleus diameter for $300 \mathrm{ppm}$ sulfur fuel 


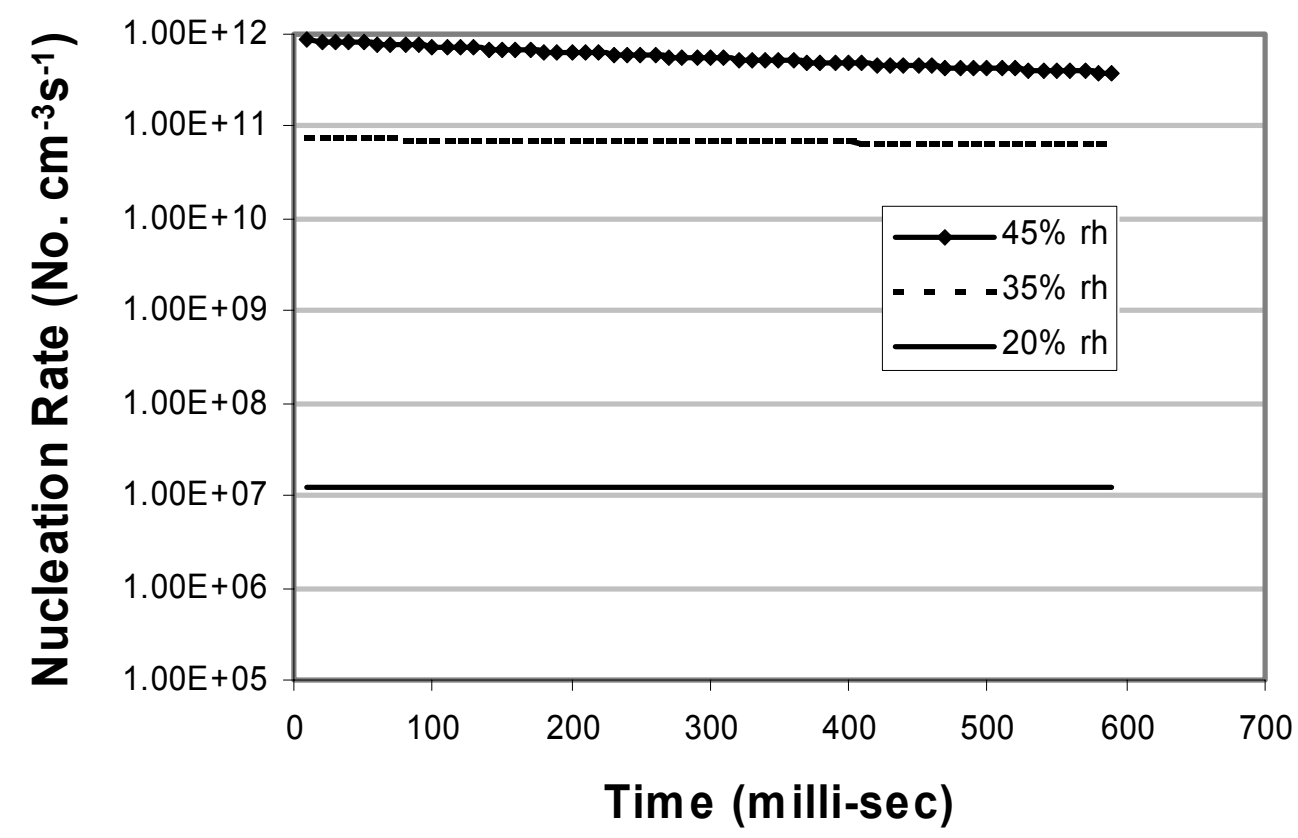

Figure 4.26 Effect of relative humidity on nucleation rate with time for $13.1 \mathrm{ppm}$ sulfur fuel 


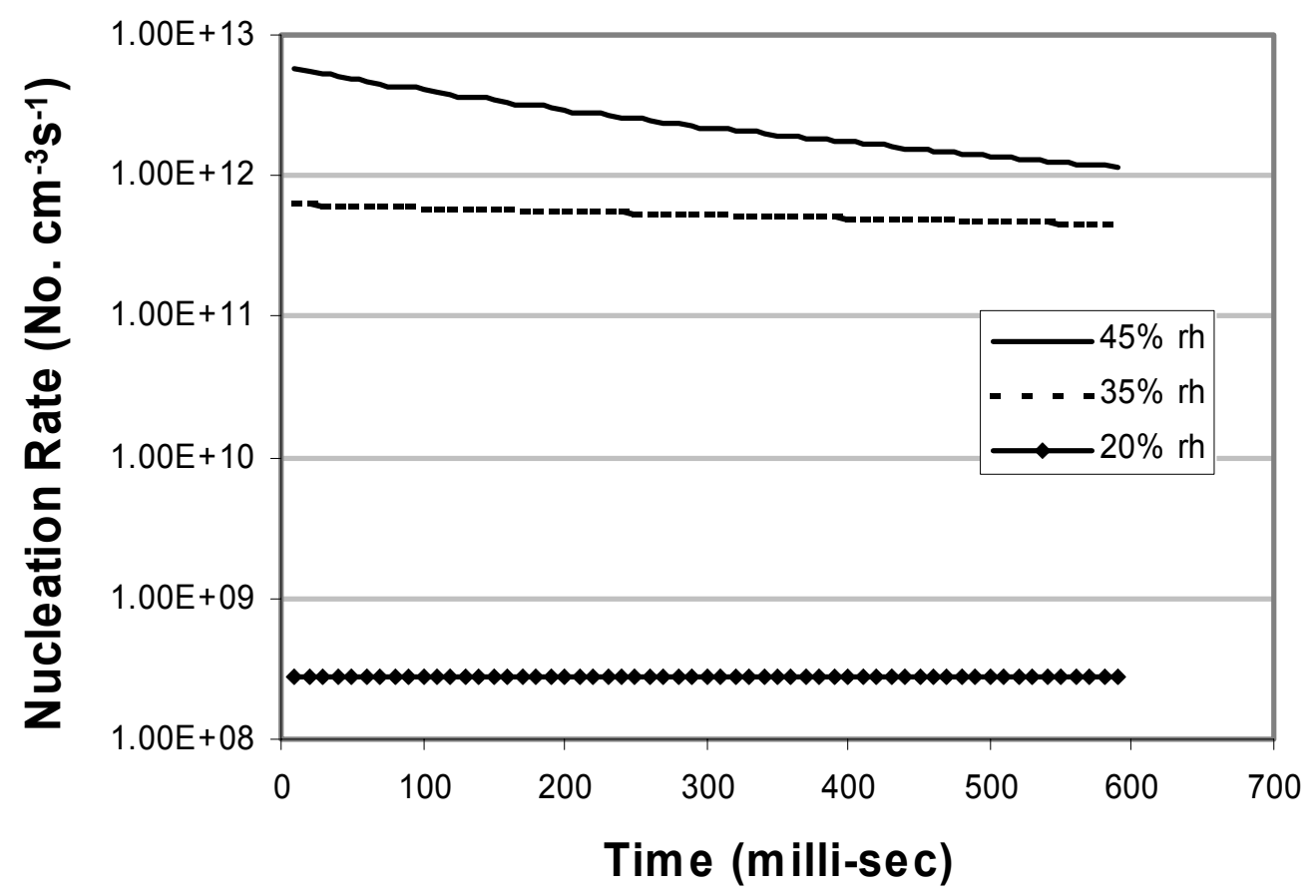

Figure 4.27 Effect of relative humidity on nucleation rate with timefor $125 \mathrm{ppm}$ sulfur fuel 


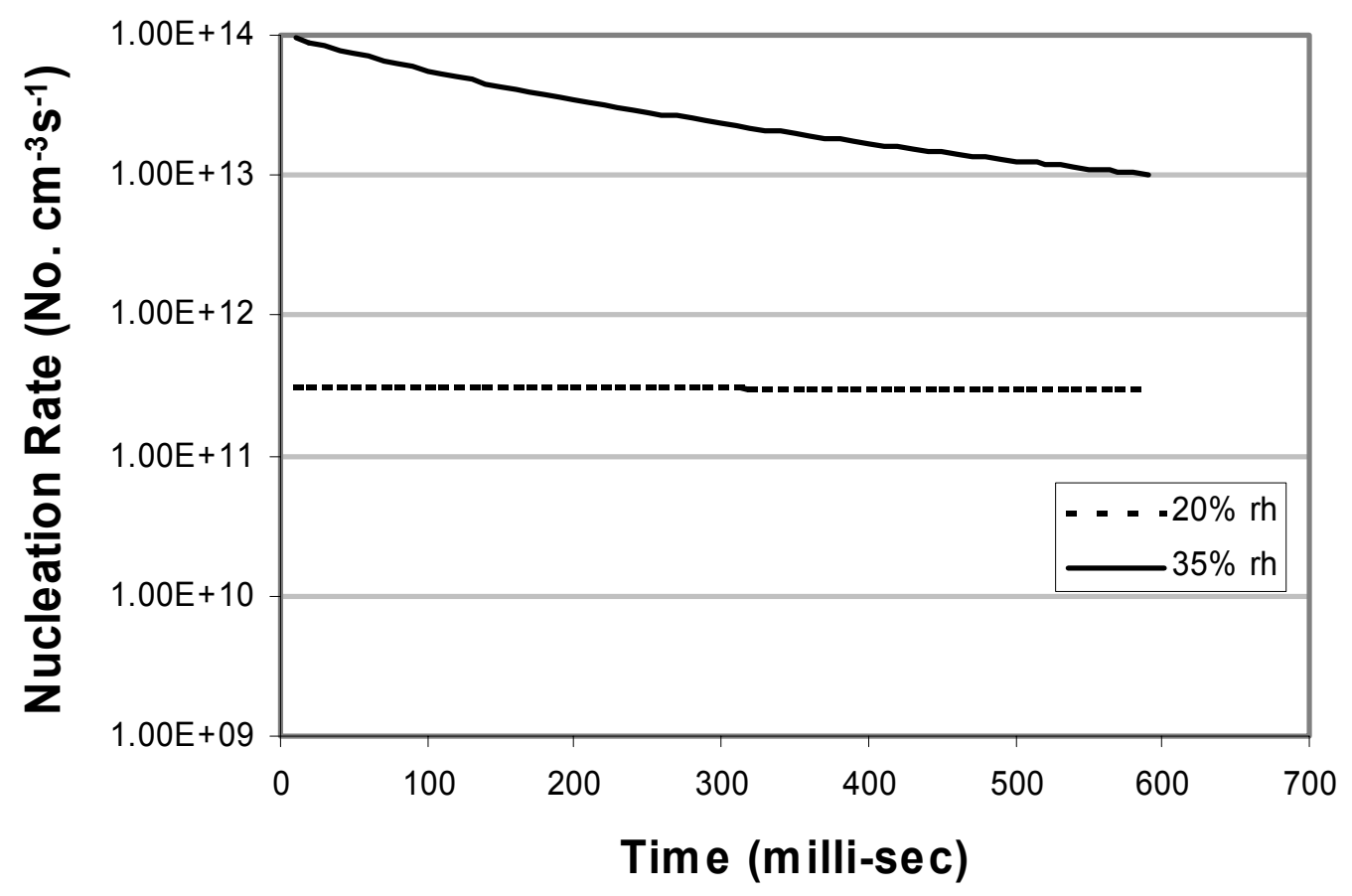

Figure 4.28 Effect of relative humidity on nucleation rate with time for $300 \mathrm{ppm}$ sulfur fuel 


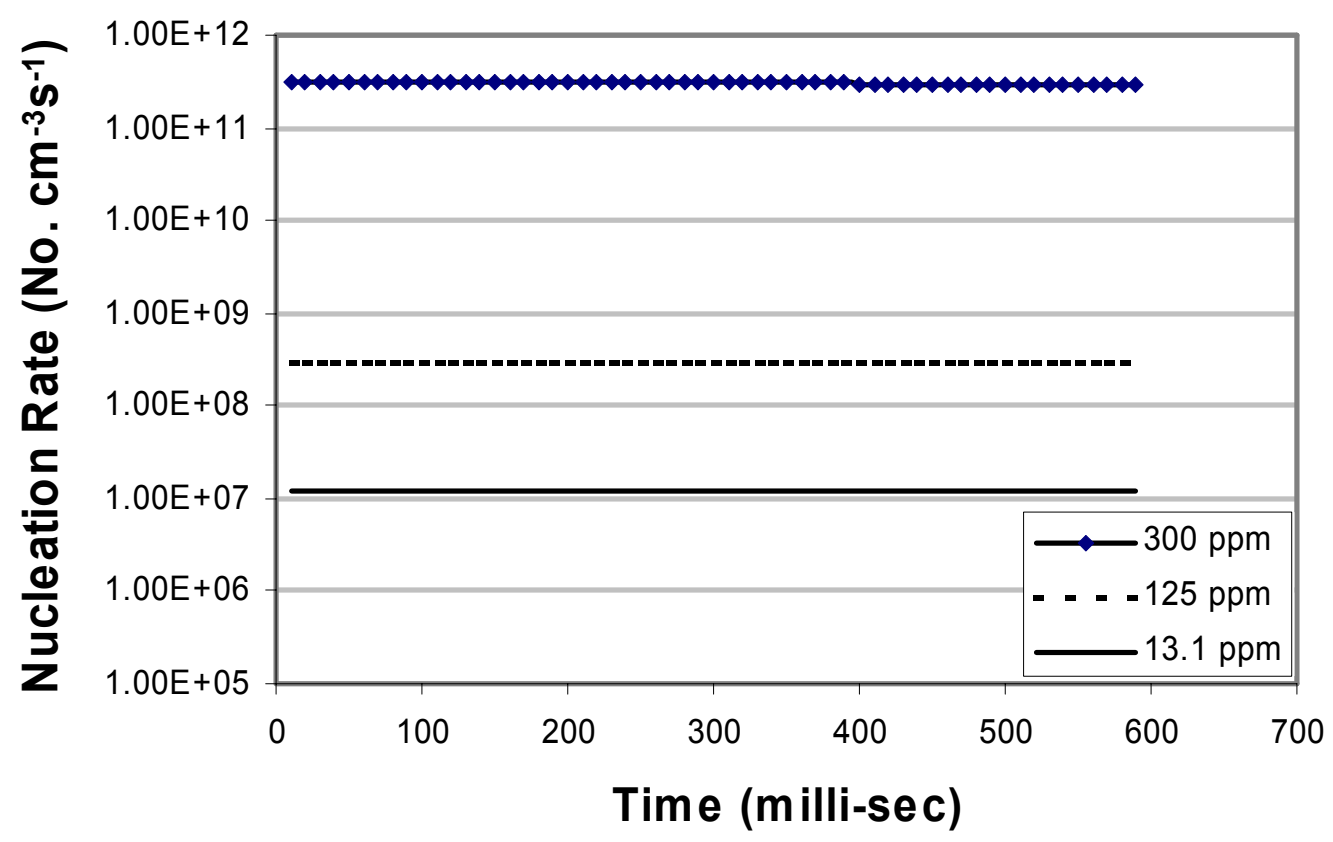

Figure 4.29 Effect of sulfur fuel content on nucleation rate with time for $20 \%$ relative humidity 


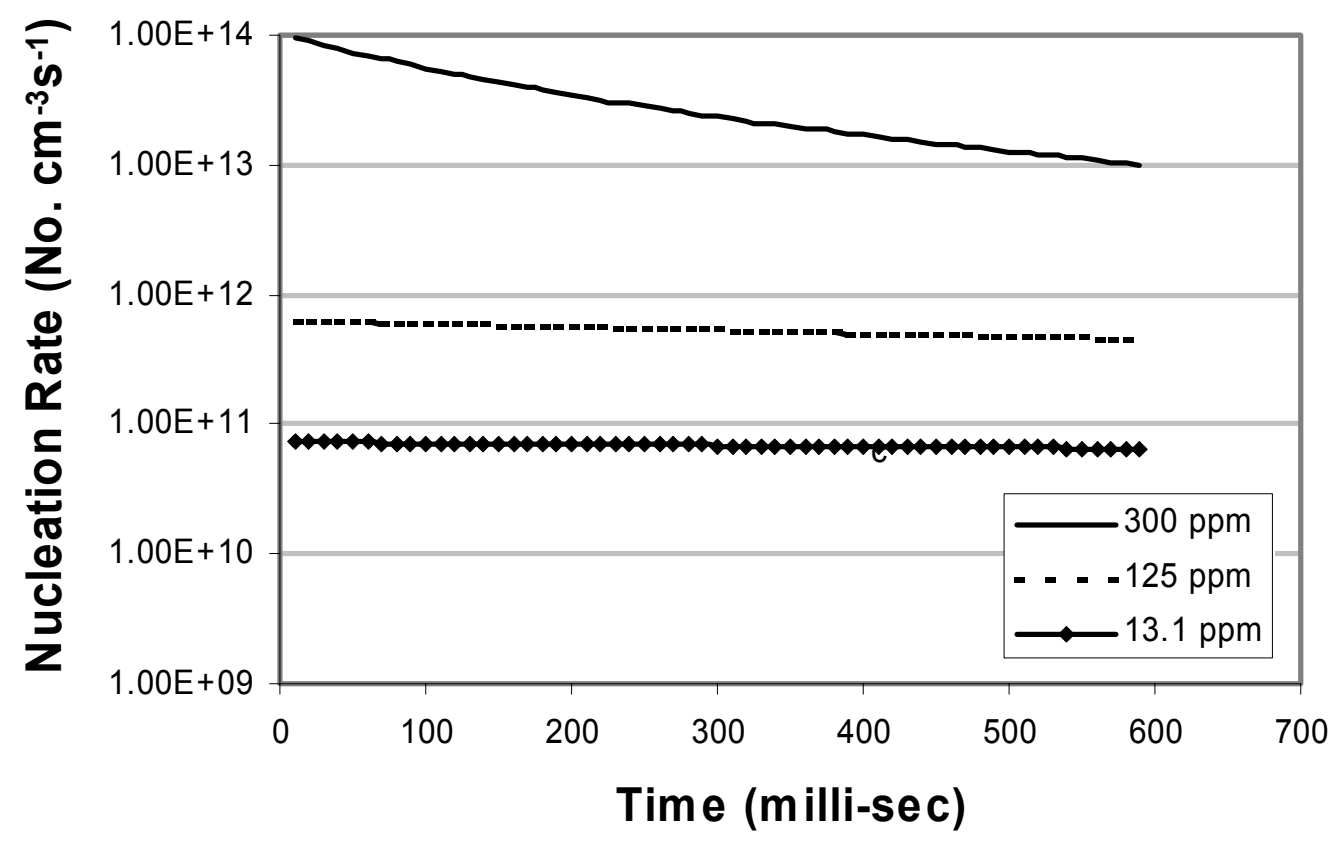

Figure 4.30 Effect of sulfur fuel content on nucleation rate with time for $35 \%$ relative humidity 


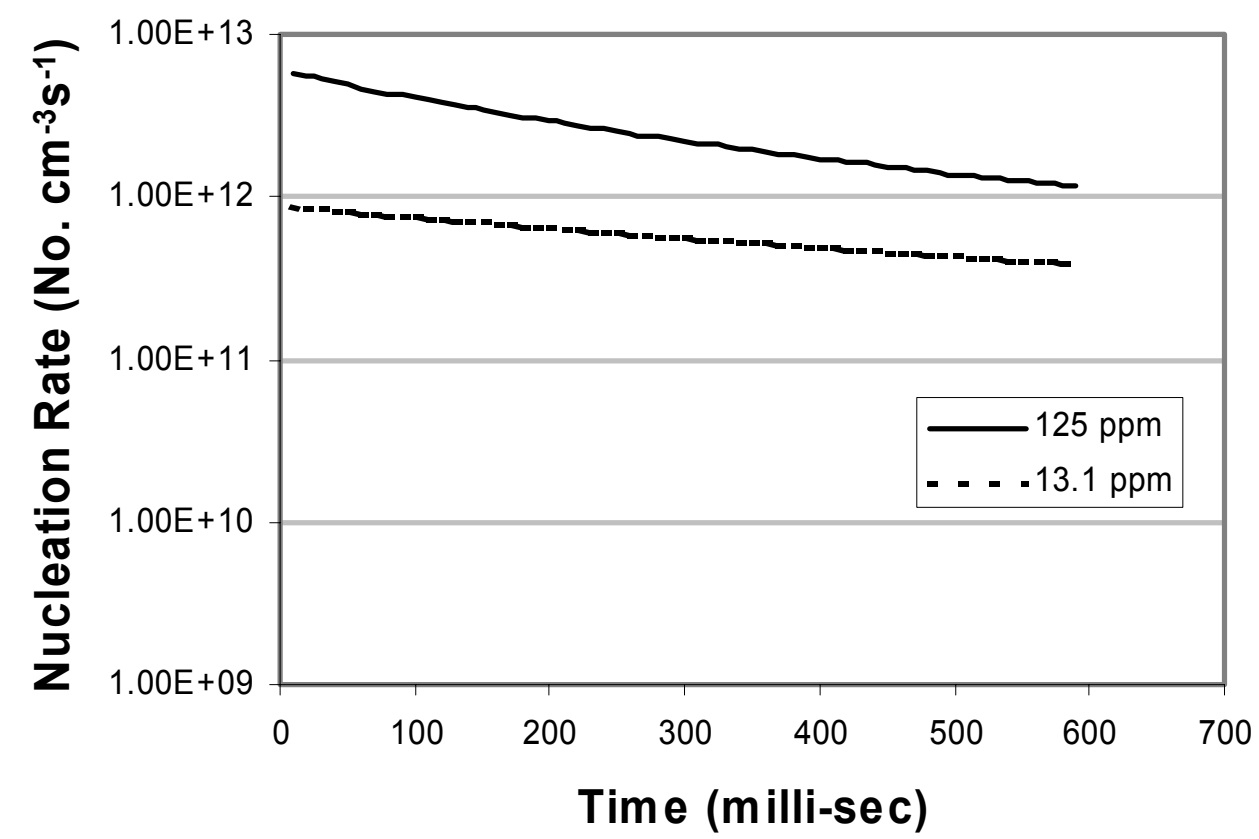

Figure 4.31 Effect of fuel sulfur content on nucleation rate with time for $45 \%$ relative humidity 


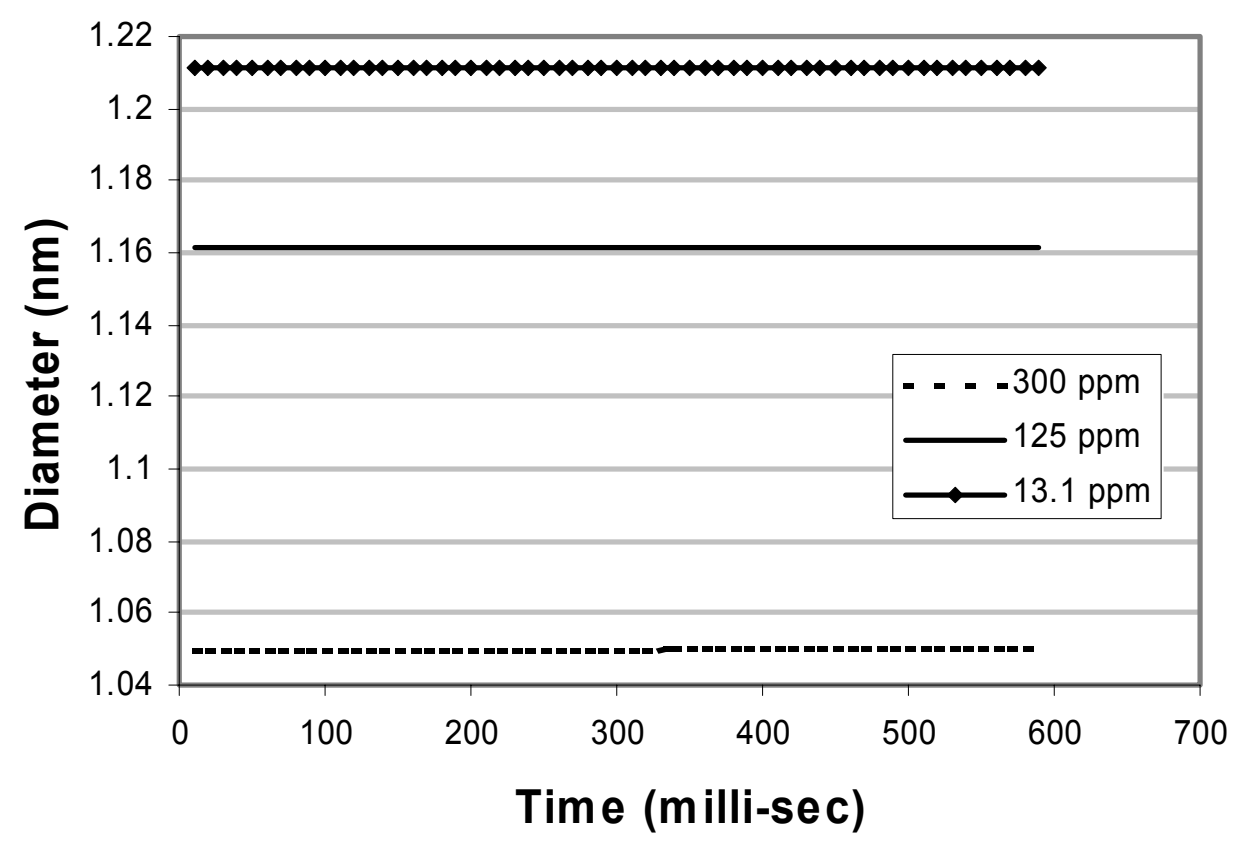

Figure 4.32 Effect of fuel sulfur content on nucleus diameter with time for $20 \%$ relative humidity 


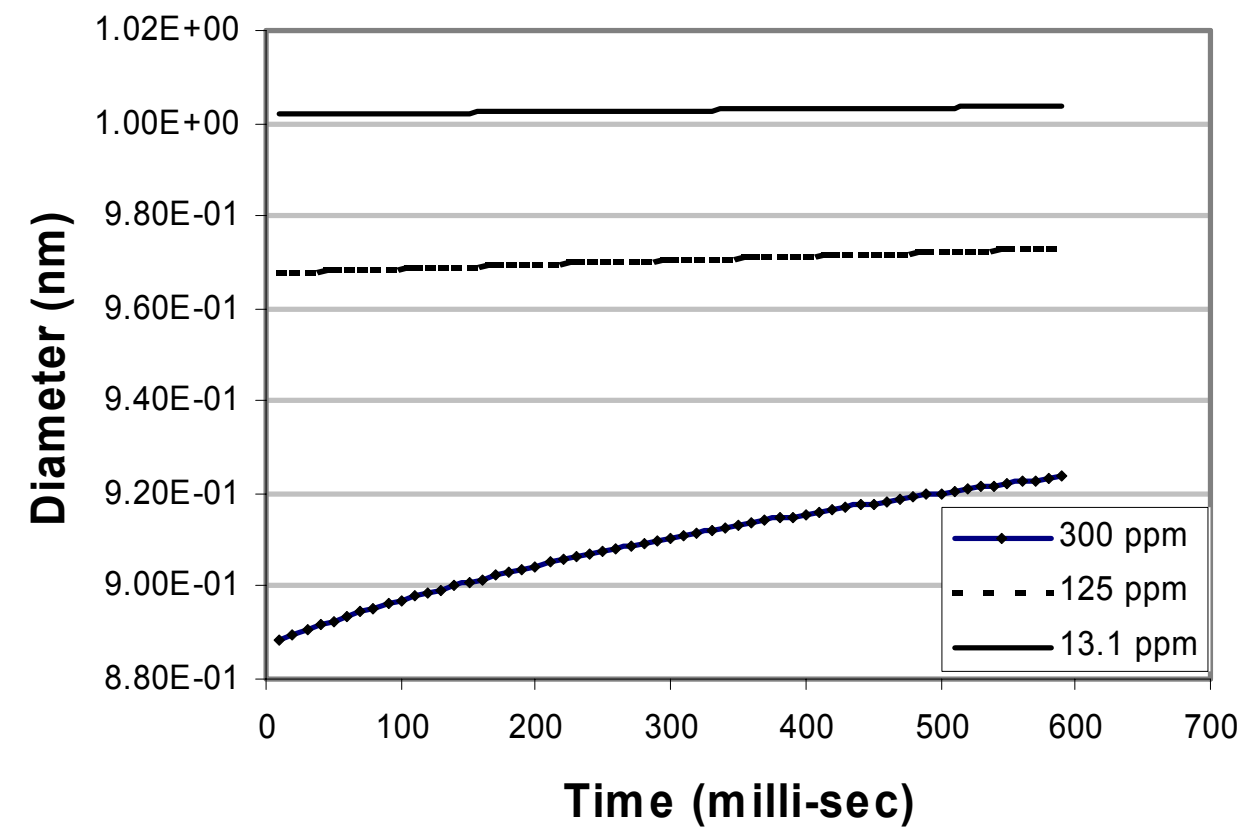

Figure 4.33 Effect of fuel sulfur content on nucleus diameter with time for $35 \%$ relative humidity 


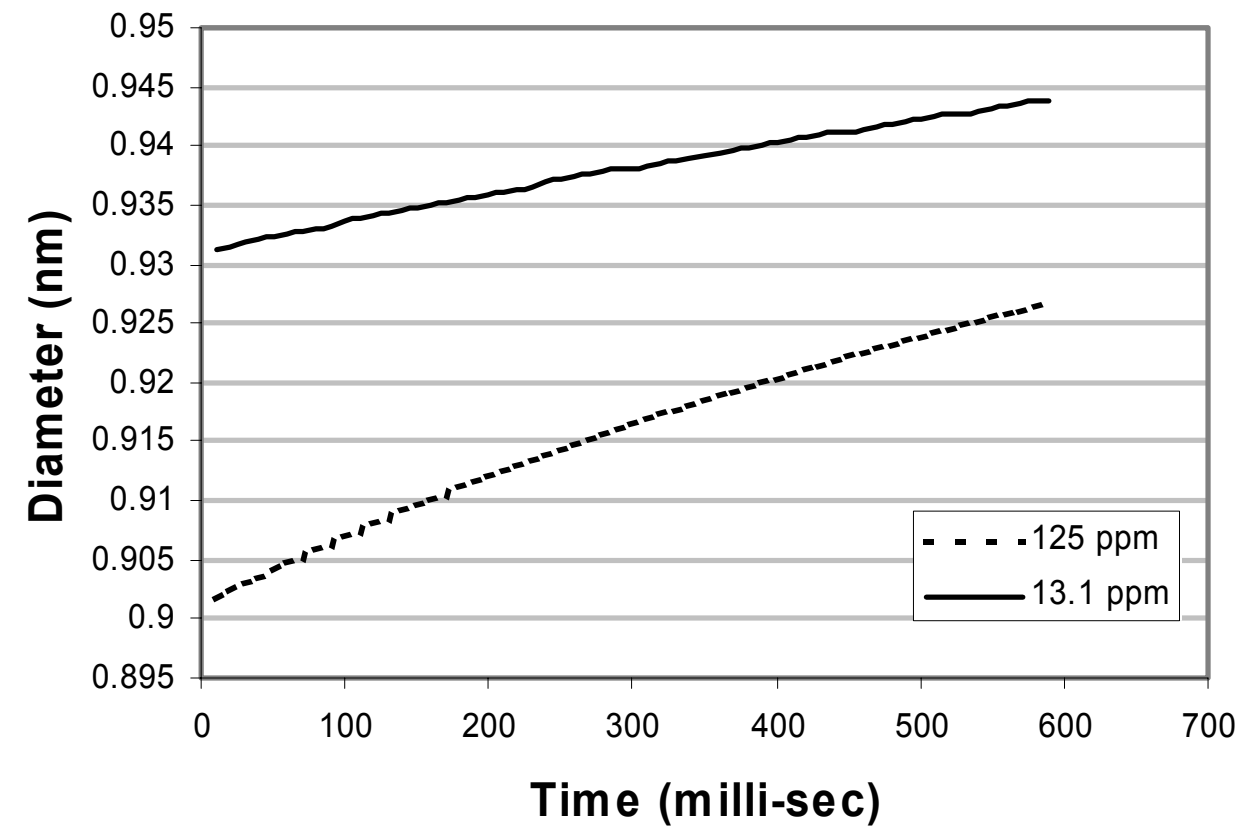

Figure 4.34 Effect of fuel sulfur content on nucleus diameter with time for $45 \%$ relative humidity 


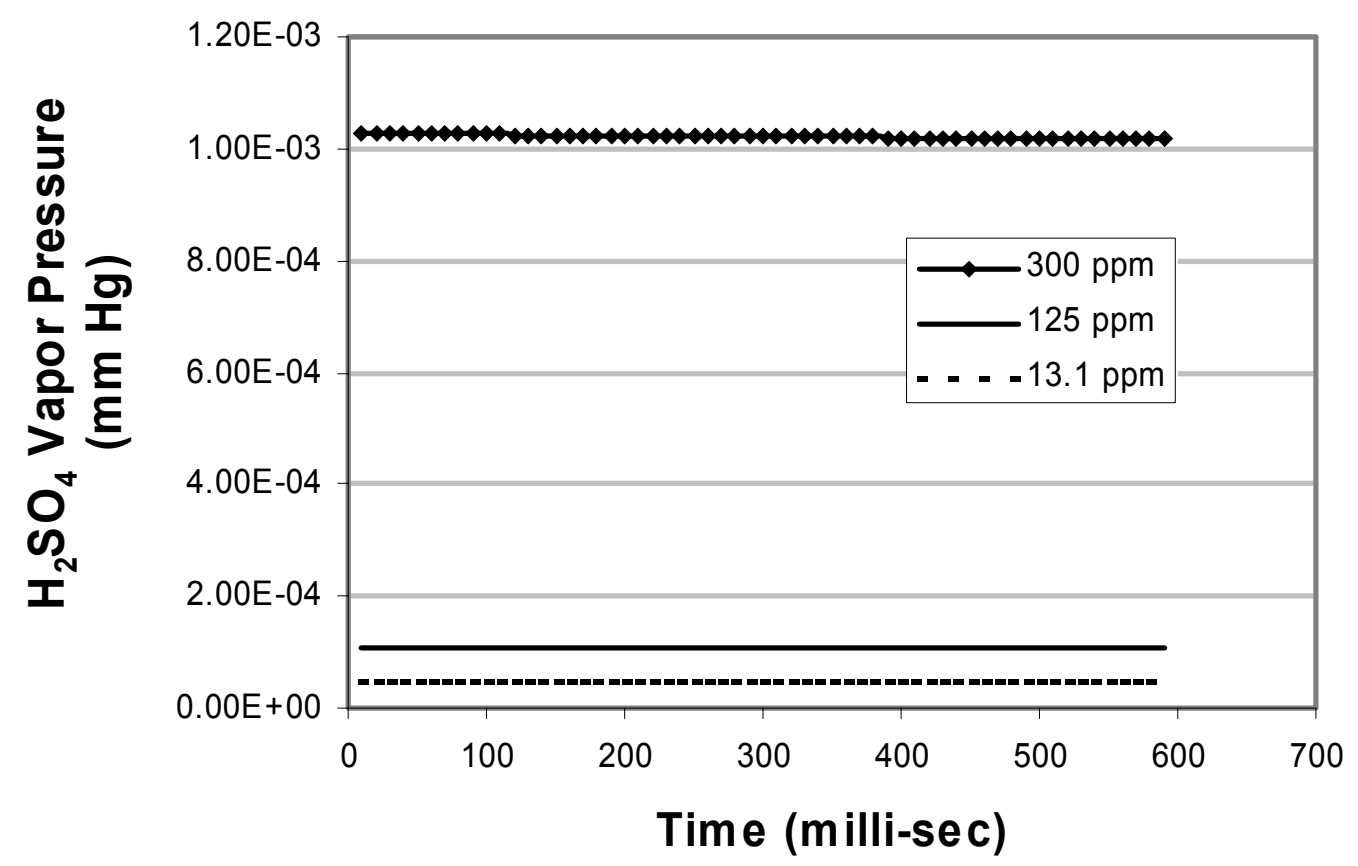

Figure 4.35 Effect of fuel sulfur content on sulfuric acid vapor pressure with time for $20 \%$ relative humidity 


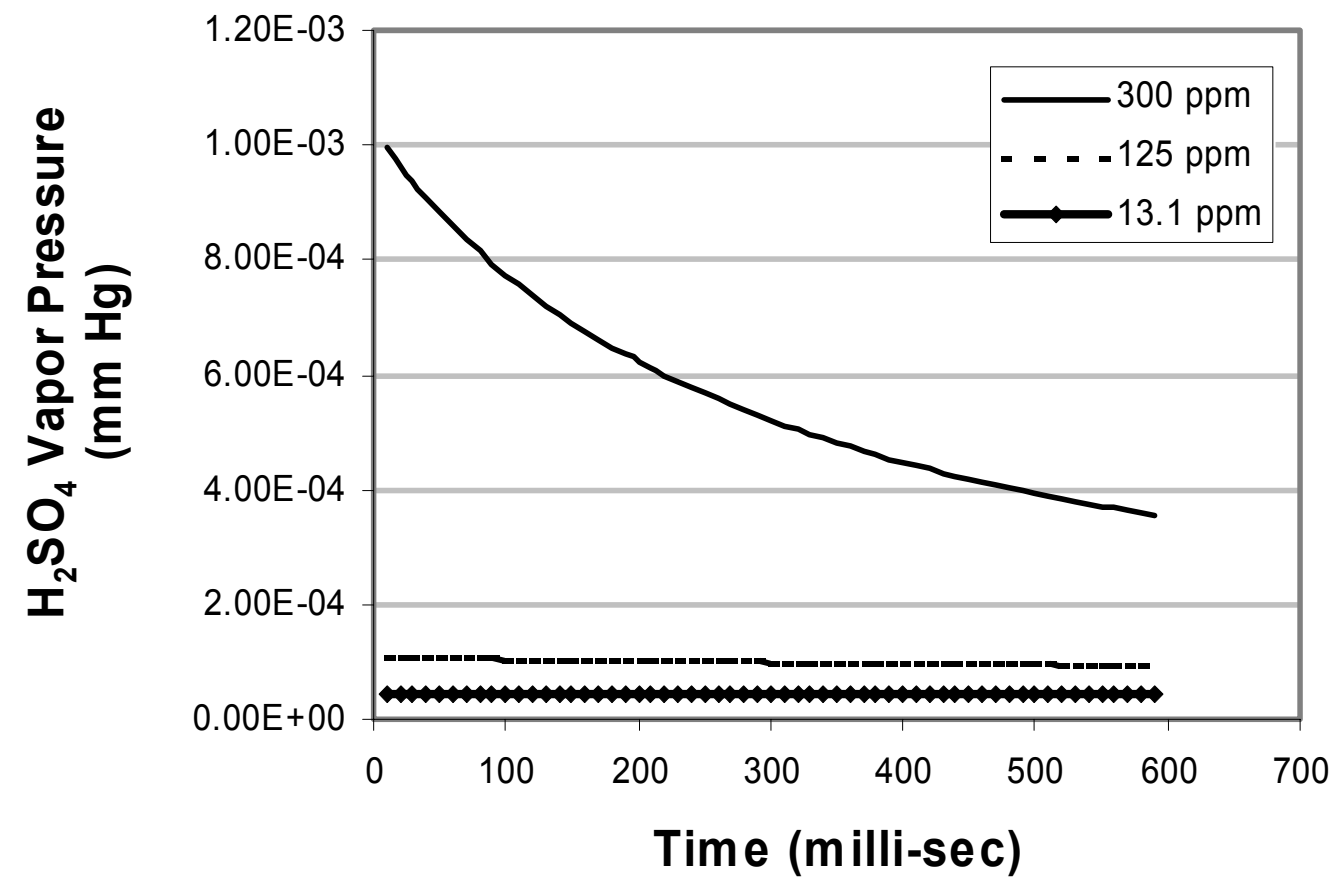

Figure 4.36 Effect of fuel sulfur content on sulfuric acid vapor pressure with time for $35 \%$ relative humidity 


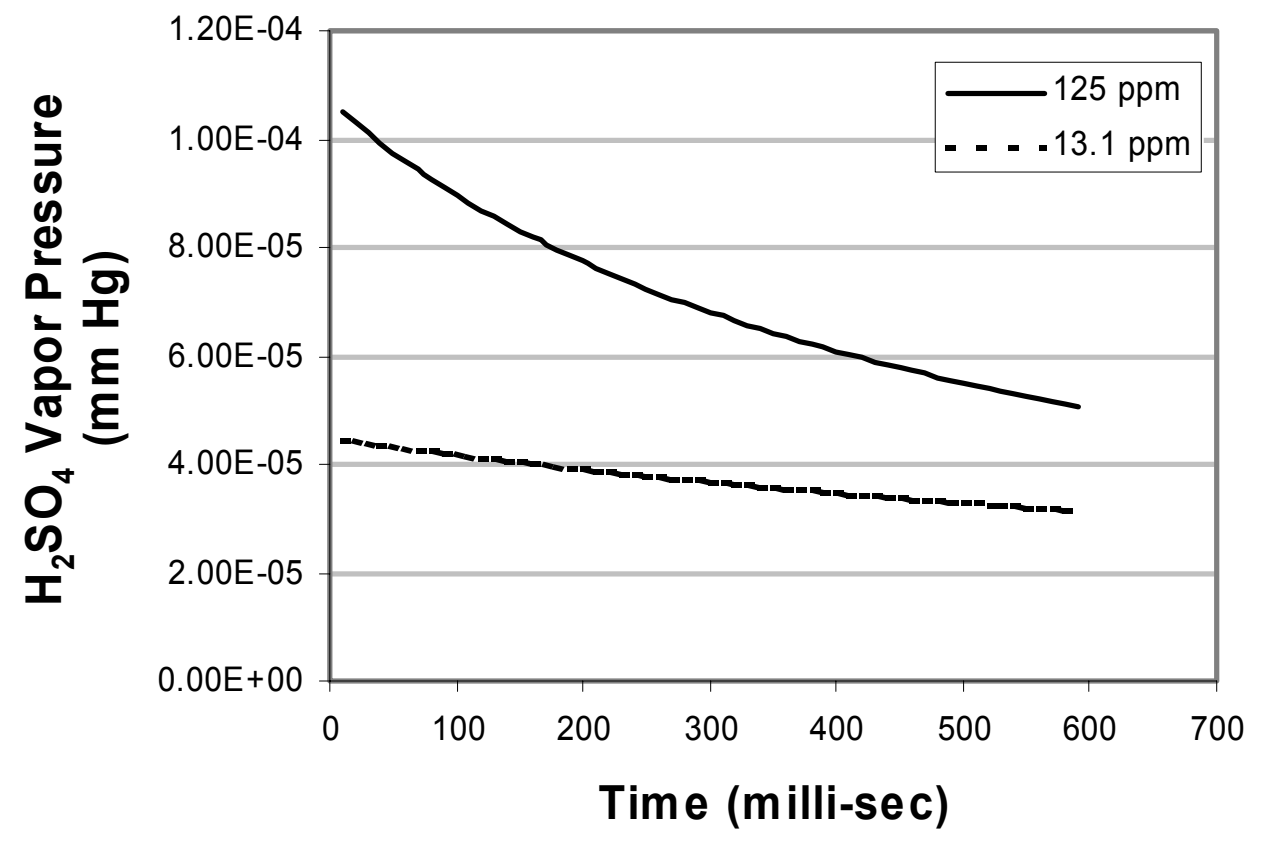

Figure 4.37 Effect of fuel sulfur content on sulfuric acid vapor pressure with time for $45 \%$ relative humidity 


\subsection{EFFECT OF COAGULATION ON PM SIZE DISTRIBUTION}

To test the coagulation model, a simple test case in the absence of any nucleation and condensation terms with an initial PM concentration of $10^{6} \mathrm{\#} / \mathrm{cm}^{3}$ was compared with the analytical solution given by Smolouchowski (Equation 3.52) for run time of $\mathrm{t}=12$ hours. The results are given in Figure 4.38. In this simple case, coagulation kernel $(\beta)$ for mono-dispersed particles is defined as:

$$
\beta=8 \mathrm{kT} / 3 \eta
$$

It may be seen that the numerical model with the volume ratio $\left(\mathrm{V}_{\mathrm{RAT}}\right)$ of 1.2 between the neighboring bins compares very well with Smolouchowski’s analytical solution.

The evolution of the particle size distribution due to coagulation is represented by Equation 3.50, which required an initial PM size distribution and concentration at the source of the plume. In the present investigation, this initial PM concentration at the source of the plume has been determined by dividing the measured values of PM concentration with the local dilution ratio at location A, 20" $(0.51 \mathrm{~m})$ apart from the source (see Figure 4.1 for its location). The PM size distributions and concentrations at three other locations B, C, and D obtained from Equation (3.51) are presented in Figure 4.39. The local dilution ratios at these 3 different locations, 80 " (2.03 m; location $\mathrm{B})$, 200" (5.08 m, location C), and 337" (8.56 m, location D) apart from the source were obtained from Figure 4.8. An excellent agreement with the measured values of PM size distribution and concentration may be viewed in Figure 4.39. 
It may be seen that the coagulation/dispersion coefficient $(\beta)$ is inversely proportional to the dynamic viscosity. Therefore, it is worthwhile to explore the effect of dynamic viscosity on the PM size distribution and concentration at different times. The results from this exploratory study are presented in Figures 4.40 and 4.41. It may be noted from Equation 3.49 that if the viscosity $(\mu)$ increases then the coagulation/dispersion coefficient ( $\beta$ ) decreases. Hypothetically, the limit $\mu$ going to zero would lead the coagulation/dispersion coefficient ( $\beta$ ) to go to infinity. Essentially, in the absence of any ambient flow (in vacuum), there will be no dispersion of particles. Hence, the particles will keep sticking to each other and theoretically, form only one big particle at the end. Alternatively, if the viscosity is high then the PM is dispersed in the ambient as soon as it is released. In the case of high viscosity, the PM distribution preserved the initial condition at different time intervals. Both of these phenomena may be observed from Figures 4.40 and 4.41. It may be seen from Figure 4.40 for smaller viscosity case, particles are sticking to each other and the particle size distribution (or count median diameter, CMD) is moving to the right side with the elapsed time. It is evident from Figure 4.41 that in a high viscosity case, the CMD is moving to right side at a much smaller rate than in Figure 4.40. It may be inferred that the dynamic viscosity plays a major role in determining the PM size distribution and concentration in the exhaust plume. 


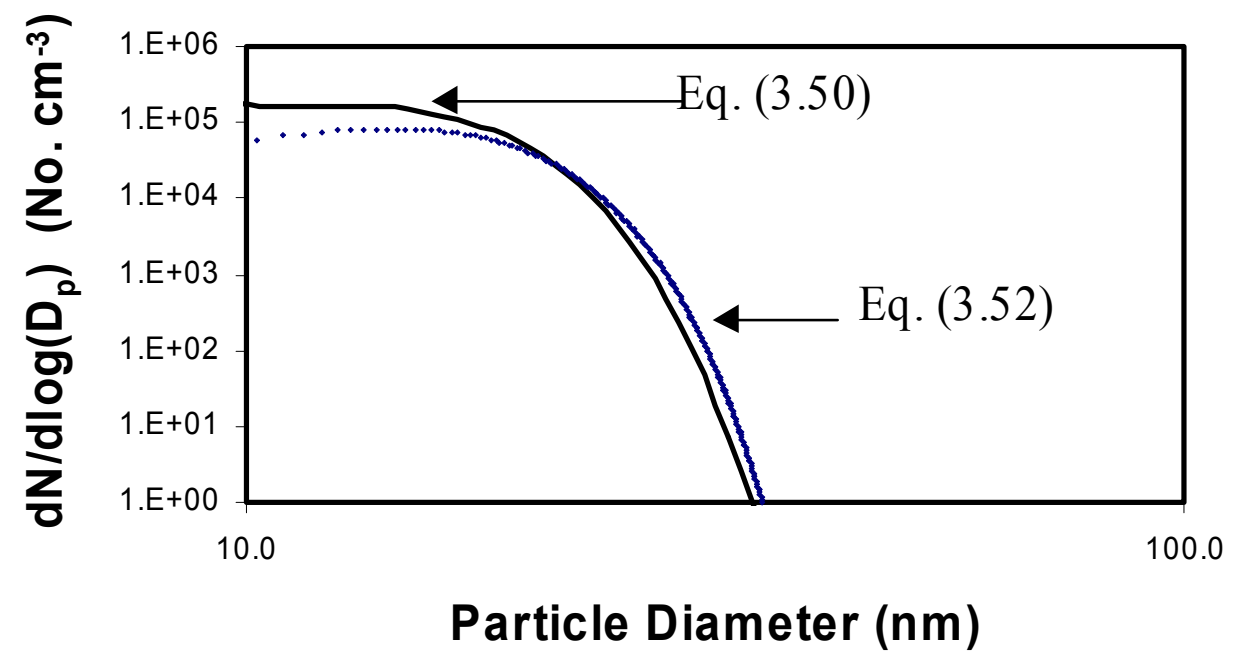

Figure 4.38 Comparison of particle concentration variation with diameter from coagulation Equation (3.50) and smoluchowski Equation (3.52) 


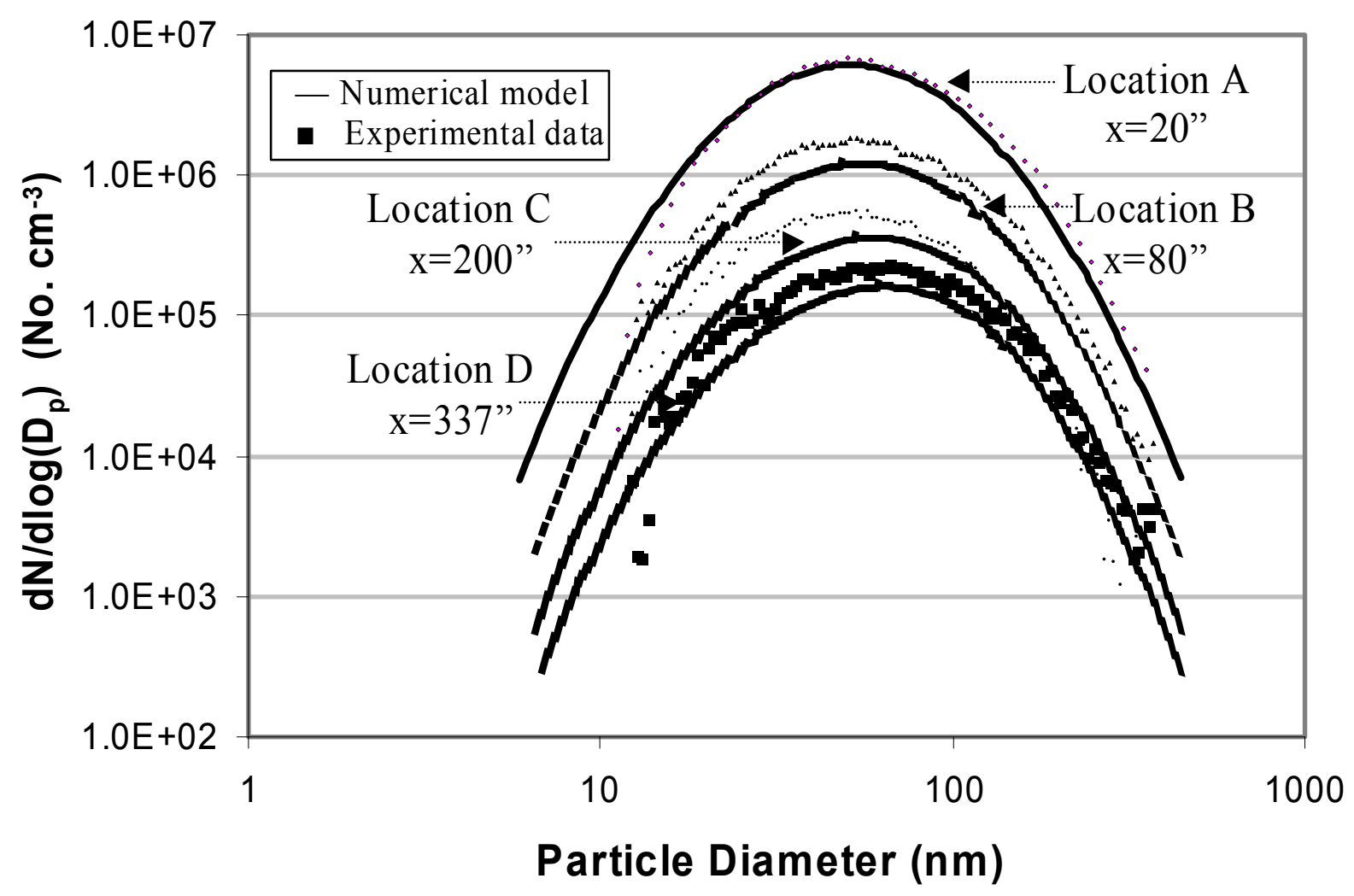

Figure 4.39 Particle Concentration Variation with Diamter at different locations for a turbulent plume 


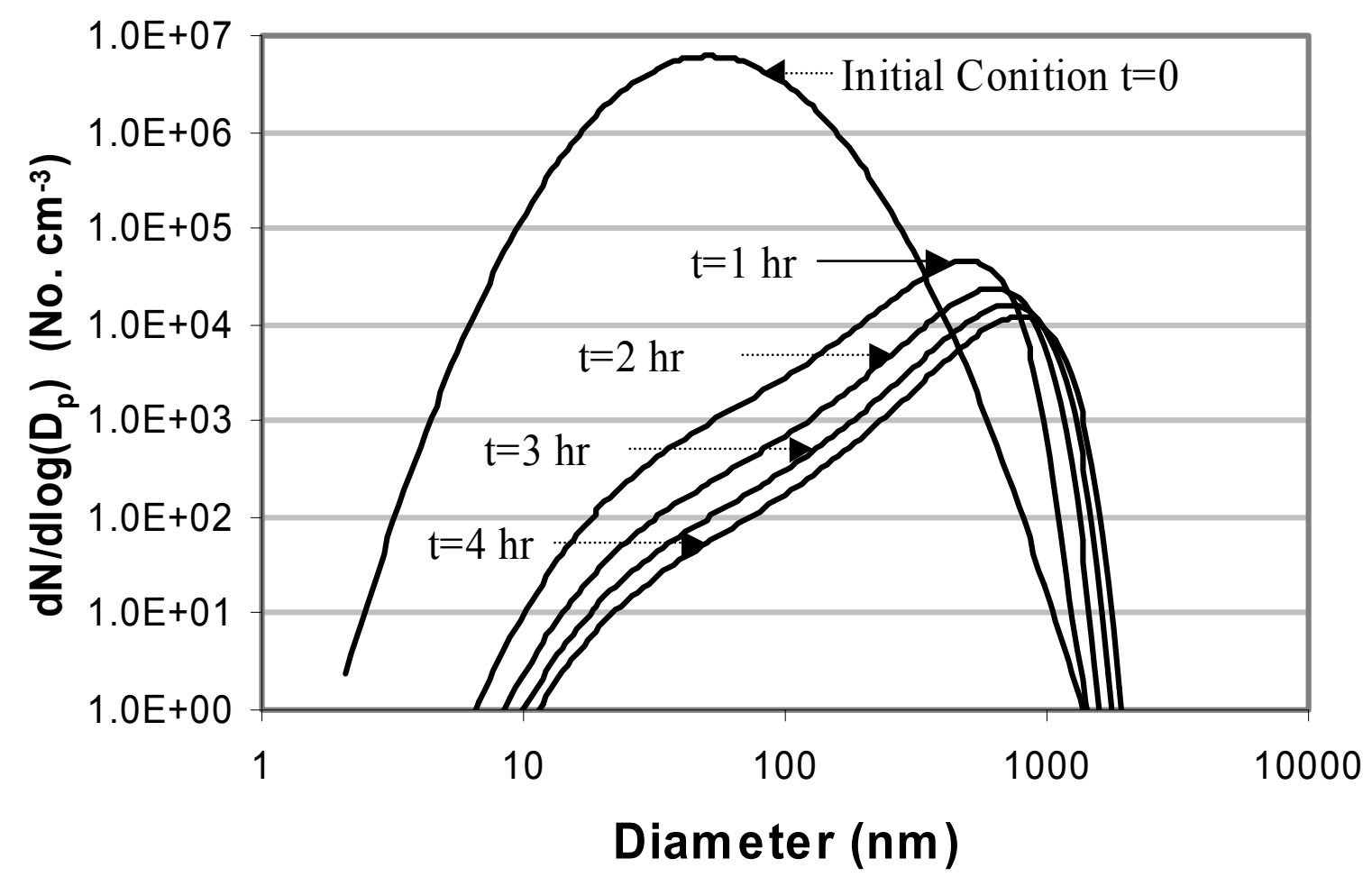

Figure 4.40 Particle concentration variation with diamter in a laminar plume at different times $\left(\mu=1.79 \mathrm{e}-4 \mathrm{dynes} \mathrm{sec} / \mathrm{cm}^{2}\right)$ 


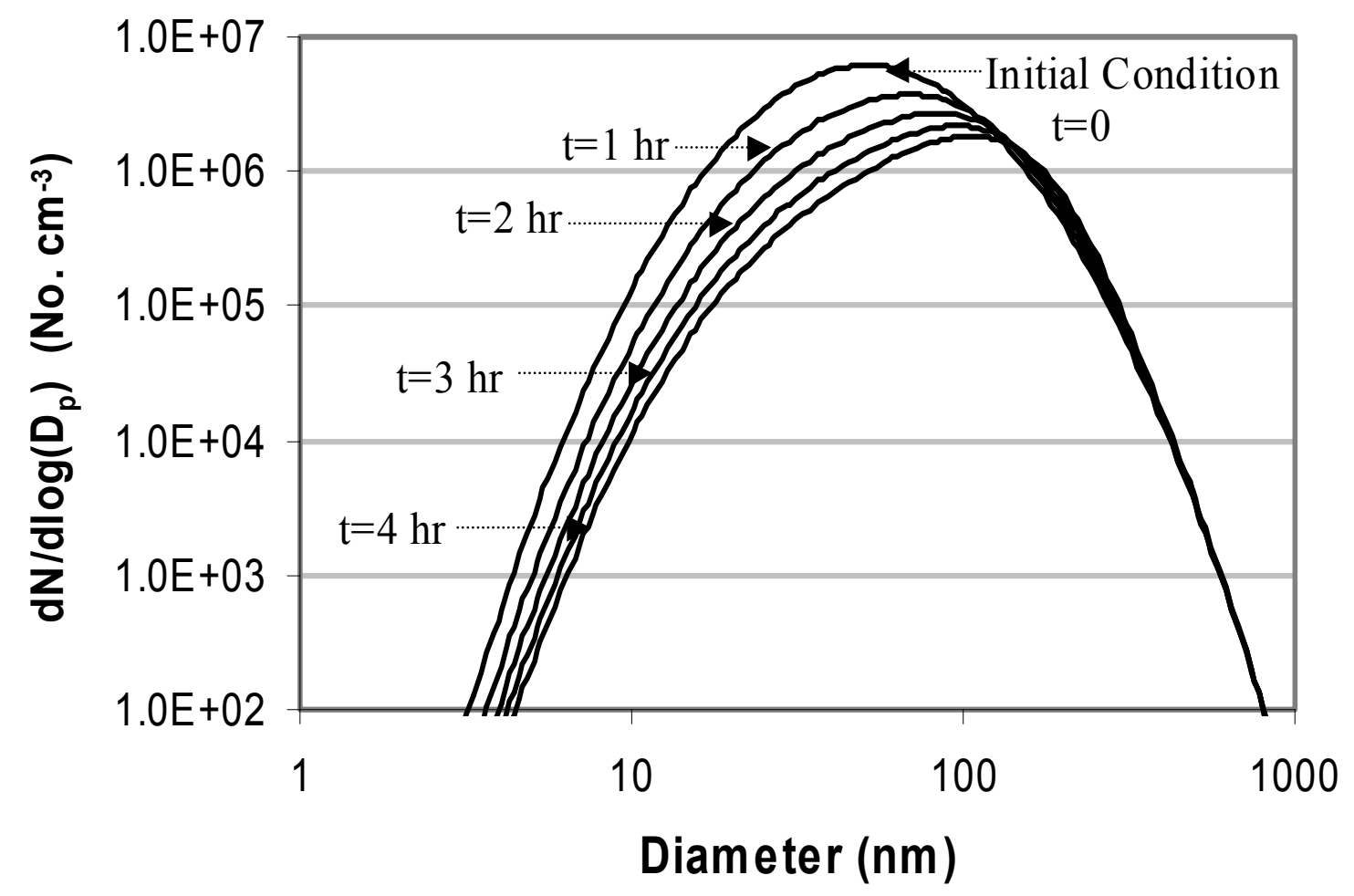

Figure 4.41 Particle concentration variation with diamter in a turbulent plume at different times $\left(\mu=5.79 \mathrm{e}-2 \mathrm{dynes} \mathrm{sec} / \mathrm{cm}^{2}\right)$ 


\subsection{EFFECTS OF NUCLEATION, CONDENSATION \& COAGULATION ON PM}

The PM size distribution and concentration obtained from Equation (3.51) with contributions from nucleation, coagulation and condensation terms at location A (20" from the stack outlet; see Figure 4.1 for location) are shown in Figure 4.42. The PM size distribution at location $\mathrm{A}$ is presented at 200 milli-seconds. The elapse of 200 milliseconds is calculated by assuming that the location $\mathrm{A}$ is approximately $5.9 \mathrm{~m}$ from the exhaust valves of the diesel engine where the nucleation process is first initiated, and the exhaust is emitted at a velocity of $29.8 \mathrm{~m} / \mathrm{s}$. In the present case, geometric ratios of 1.4, 1.2, and 1.05 were employed to check the grid independent solutions. It was found that there was no significant difference in the results obtained from the geometric ratios of 1.2 and 1.05 . Hence, the geometric ratio of 1.05 was used for the discretization of bin sizes in the current computations. It may be also be seen from Figure 4.42 that if the contribution from condensation term is included then the PM count median diameter (CMD: inferred from the peak diameter) shifts to the right from approximately $10 \mathrm{~nm}$ to $52 \mathrm{~nm}$. This shift may be attributed to the fact that the condensation essentially increases the nucleus radius. When the nucleus size increases, then it is worth mentioning that the coagulation/agglomeration process is enhanced because these nuclei particles with their high diffusion coefficients diffuse to large particles with large surface area. This also implies that near the stack outlet, where rapid dilution (see also Figure 4.8) of exhaust with the ambient air is taking place, condensation effects are very important. Additionally, the dip near $10 \mathrm{~nm}$ in Figure 4.42 indicates that the nucleation mode (typically less than $10 \mathrm{~nm}$ ) is dominant, and the coagulation/agglomeration of particles is causing the particle diameter to increase. It should be noted that experimental data for 
PM size less than $10 \mathrm{~nm}$ is not believed to be reliable by WVU researchers. Although the instrument manufacturer claims as lower size limit, the SMPS configuration that was used in the Langley tunnel is limited in its measurement capability to $10 \mathrm{~nm}$ at the lower end (Bischof, 1998). The count median diameter predicted by the numerical model is 52 $\mathrm{nm}$, and the CMD measured experimentally is $51 \mathrm{~nm}$. The root mean square (RMS) error in the predicted particle number concentration with respect to the experimentally measured values is $14.3 \%$. The RMS error for the particle number concentration is calculated as:

$R M S$ Error (Particle Concentration $)=\frac{\frac{\sqrt{\left(\sum_{i=1}^{N_{B}}\left(N_{i, \text { numerical }}-N_{i, \text { measured }}\right)^{2}\right)}}{N_{B}-1}}{N_{\text {Maximum at CMD }}}$

where $\mathrm{N}_{\mathrm{i}}$, numerical is the numerically predicted particle concentration, $\mathrm{N}_{\mathrm{i}}$, measured is the experimentally measured particle concentration, and the RMS error is normalized by the maximum particle concentration that exists at the CMD value.

The predicted PM size distribution and concentration profiles obtained from Equation (3.51) at three additional locations B, C, and D are compared to experimental results in Figure 4.43. The local dilution ratios at these 3 different locations, 80" (2.03 m; location B), 200" (5.08 m, location C), and 337" (8.56 m, location D) from the source were obtained from Figure 4.43. A good agreement of the results with the measured values of PM size distributions and concentrations may be viewed in Figure 4.43. Part of 
discrepancy on the right tail of the distribution may be due to the fact that the current model does not include the contribution from the dry soot/unburned carbon from the fuel.

The effects of different sulfur level (13.1 ppm and $125 \mathrm{ppm})$ on particle diameter and concentration for constant relative humidity of 30\% are shown in Figure 4.44 and Figure 4.45 , respectively. It may be seen that the model predictions agree well qualitatively with the experimentally measured values at 0.14 and 0.17 seconds.

Figures 4.46 and 4.47 present the effect of different sulfur fuel content (13.1 ppm and $125 \mathrm{ppm}$ ) on particle diameter and concentration at $0.14 \mathrm{sec}$ and $0.34 \mathrm{sec}$, respectively. It is worth mentioning that the count median diameter (CMD) for fuel with sulfur content of $125 \mathrm{ppm}$ shifted to right more quickly than fuel with sulfur content of 13.1 ppm. This may be due to the fact that higher fuel sulfur concentration results in higher $\mathrm{SO}_{4}$ concentration in the exhaust. As particles grow, the $\mathrm{H}_{2} \mathrm{SO}_{4}$ particle molar fraction decreases due to the addition of $\mathrm{H}_{2} \mathrm{O}$ molecules, and the vapor pressure above the particles surface decreases. When the particle's $\mathrm{H}_{2} \mathrm{SO}_{4}$ or $\mathrm{H}_{2} \mathrm{O}$ vapor pressure equals the species atmospheric vapor pressure, the number of molecules striking the particles surface equals the number of molecules leaving the surface, and the particle diameter will become stable. Also, a bigger critical diameter of particles will result in much faster growth of the particles because of coagulation.

The particulate matter concentrations predicted by the model for the different sulfur fuel contents (13.1 and $300 \mathrm{ppm}$ ) at a constant relative humidity of $30 \%$ are shown 
in Figure 4.48. The residence time of $0.14 \mathrm{sec}$ was used for calculation in the Figure 4.48. It may be noted that the curve for high sulfur fuel $(300 \mathrm{ppm})$ shifted to the right more quickly than low sulfur fuel (13.1 ppm).

The effects of relative humidity on particulate matter size distribution and concentrations are shown in Figure 4.49. The results of simulation for fuel with sulfur content of 125 ppm are shown in Figure 4.49. It may be noted that the CMD for the case of higher relative humidity (48\%) shifted to the right (larger CMD) more quickly than the case with a relative humidity of $30 \%$. 


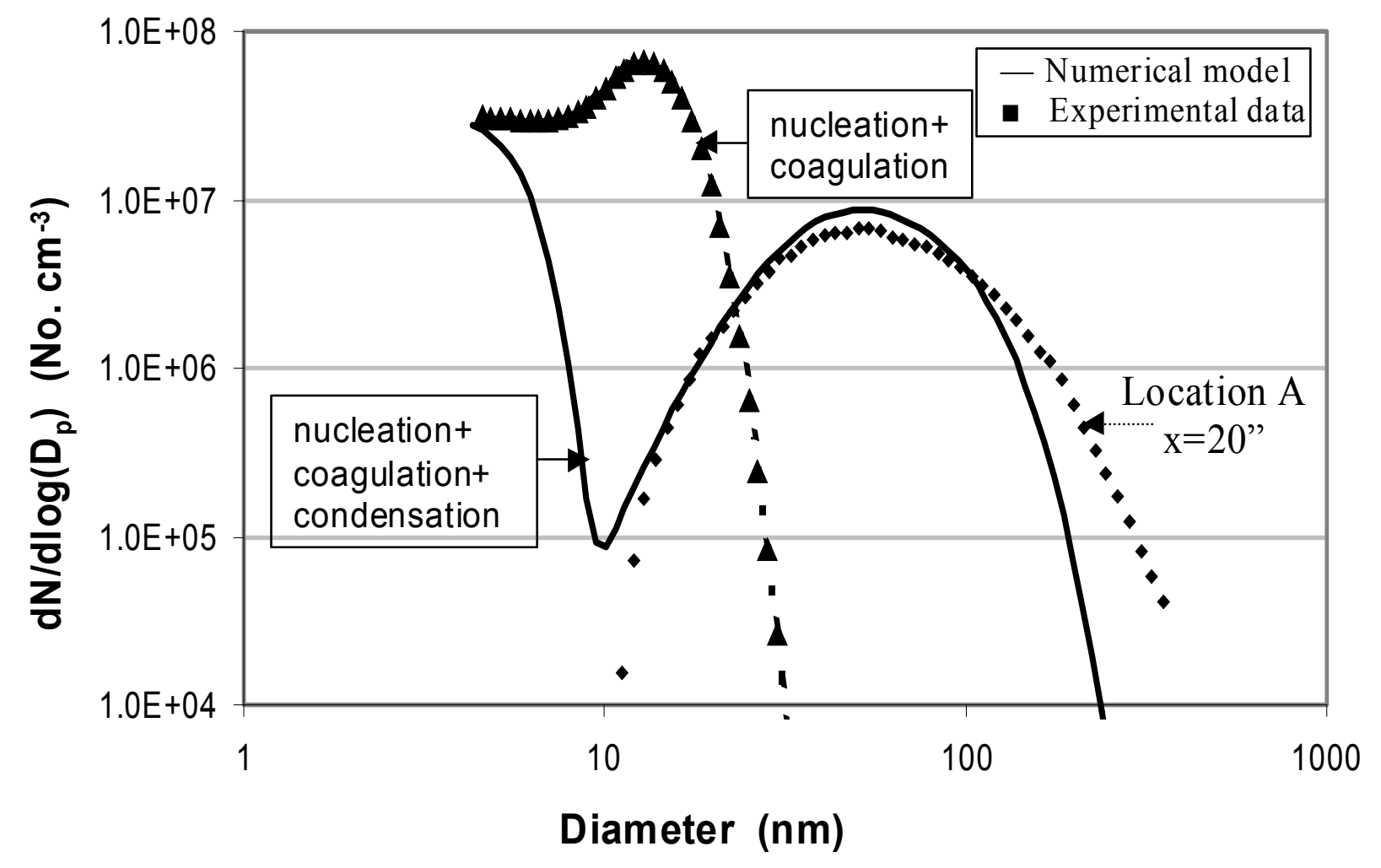

Figure 4.42 Particle concentration variation with diameter at a location 20 " $(0.5 \mathrm{~m})$ from the stack outlet by considering (a) nucleation+coagulation $(\boldsymbol{\Lambda})$, and (b) nucleation+coagulation+ condensation effects 


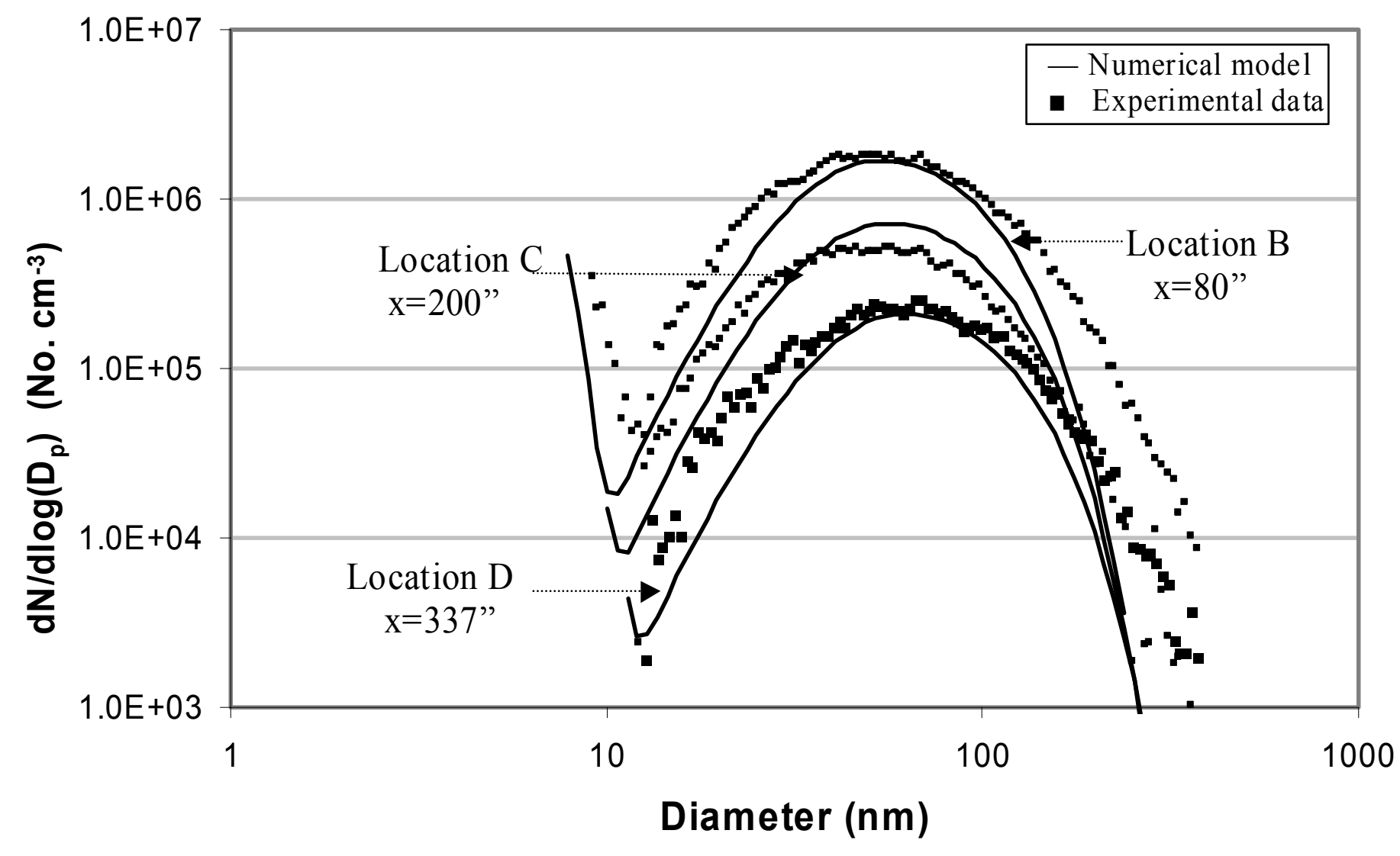

Figure 4.43 Particle concentration variation with diameter at different locations in a turbulent plume using nucleation+coagulation+condensation model 


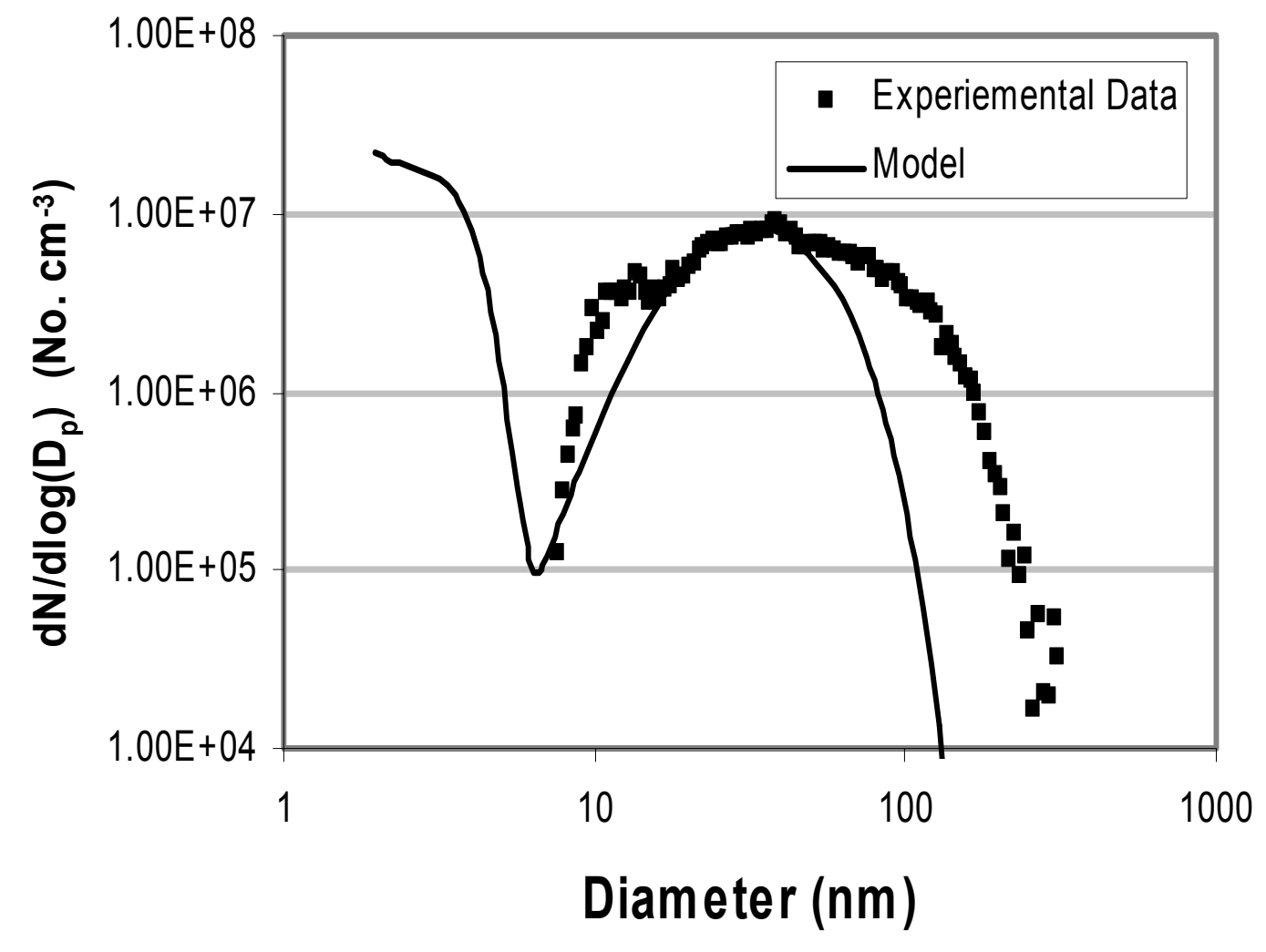

Figure 4.44 Comparison with experiemntal data of particle concentration variation with diameter (sulfur fuel level $=13.1 \mathrm{ppm}$, relative humidity $=30 \%$, dilution ratio $=10$ ) 


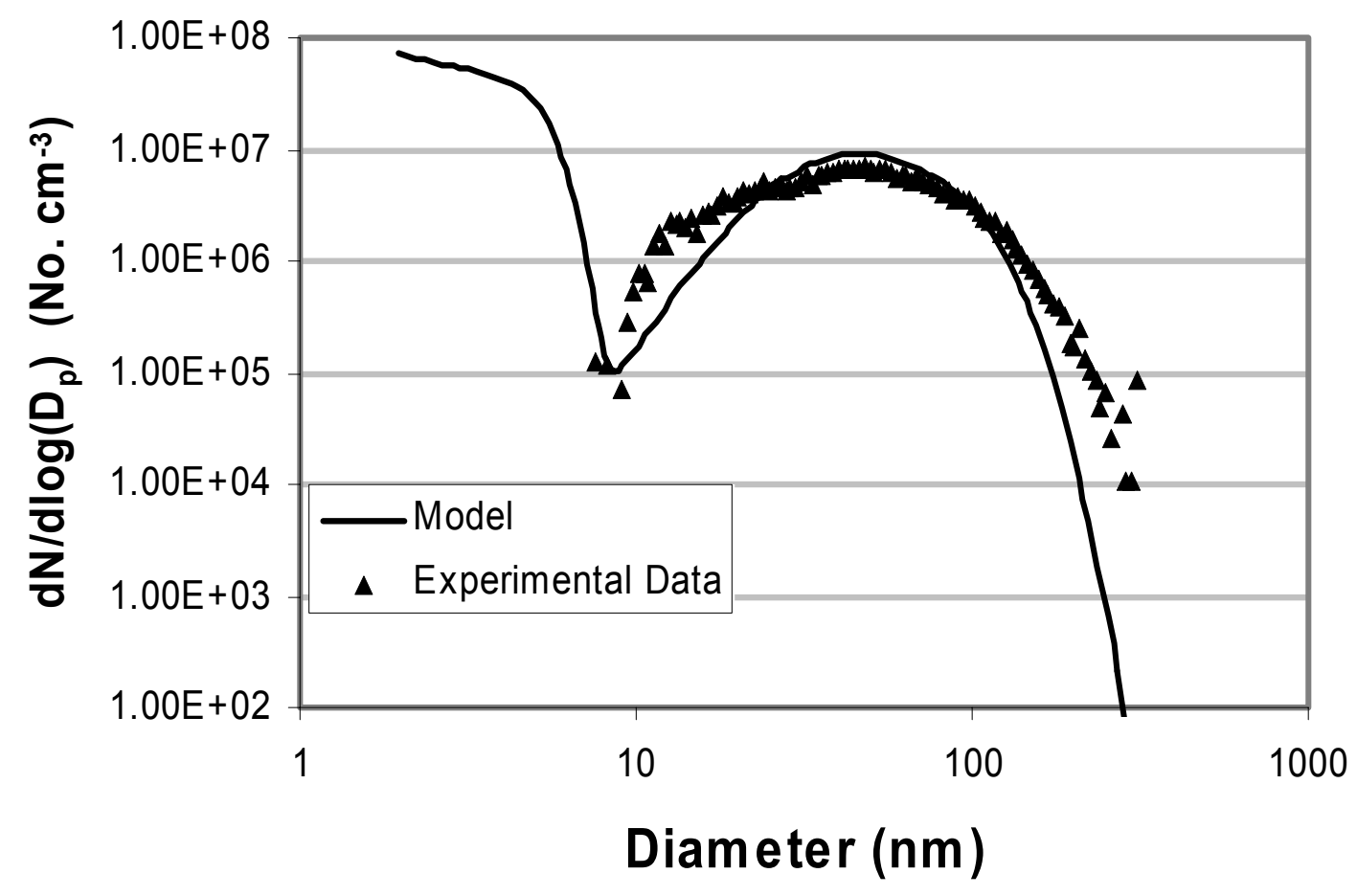

Figure 4.45 Comparison with experiemntal data of particle concentration variation with diameter (sulfur fuel level $=125 \mathrm{ppm}$, relative humidity $=30 \%$, dilution ratio $=5$ ) 


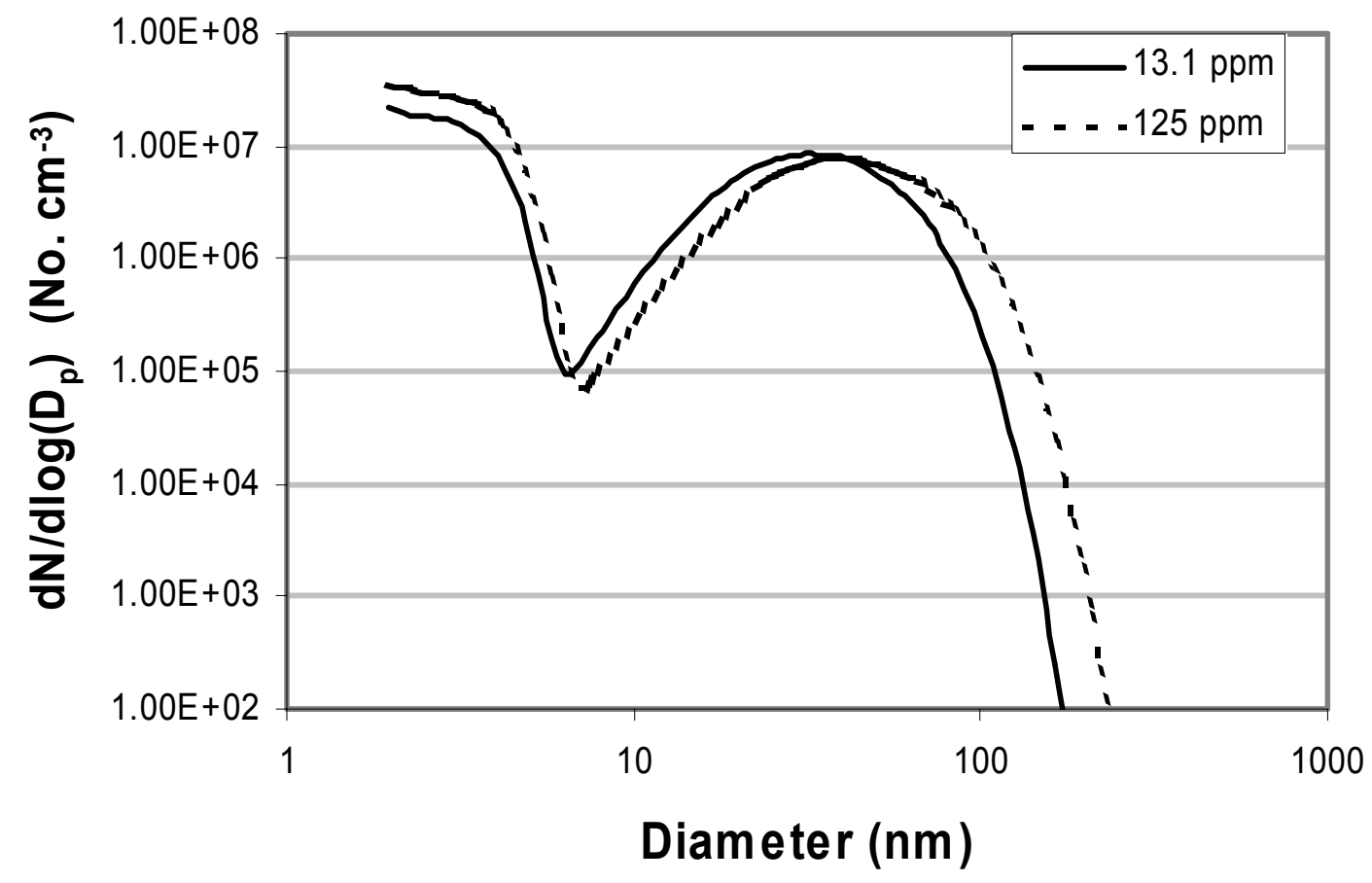

Figure 4.46 Particulate matter prediction on different sulfur fuel level (13.1 and $125 \mathrm{ppm})$ at $0.14 \mathrm{sec}(30 \%$ relative humidity, dilution ratio 10$)$ 


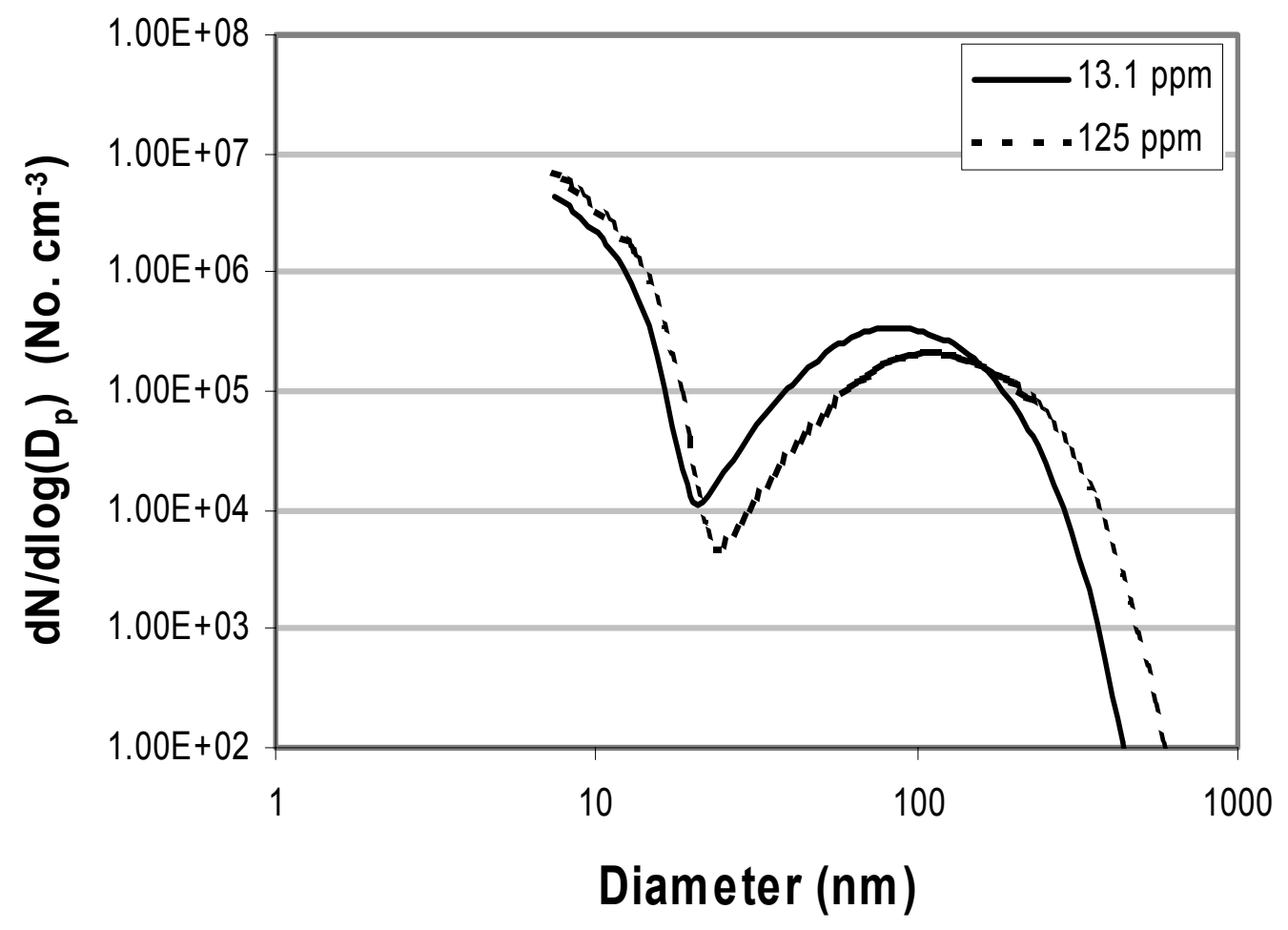

Figure 4.47 Particulate matter prediction on different sulfur fuel level (13.1 and $125 \mathrm{ppm})$ at $0.34 \mathrm{sec}(30 \%$ relative humidity, dilution ratio 10$)$ 


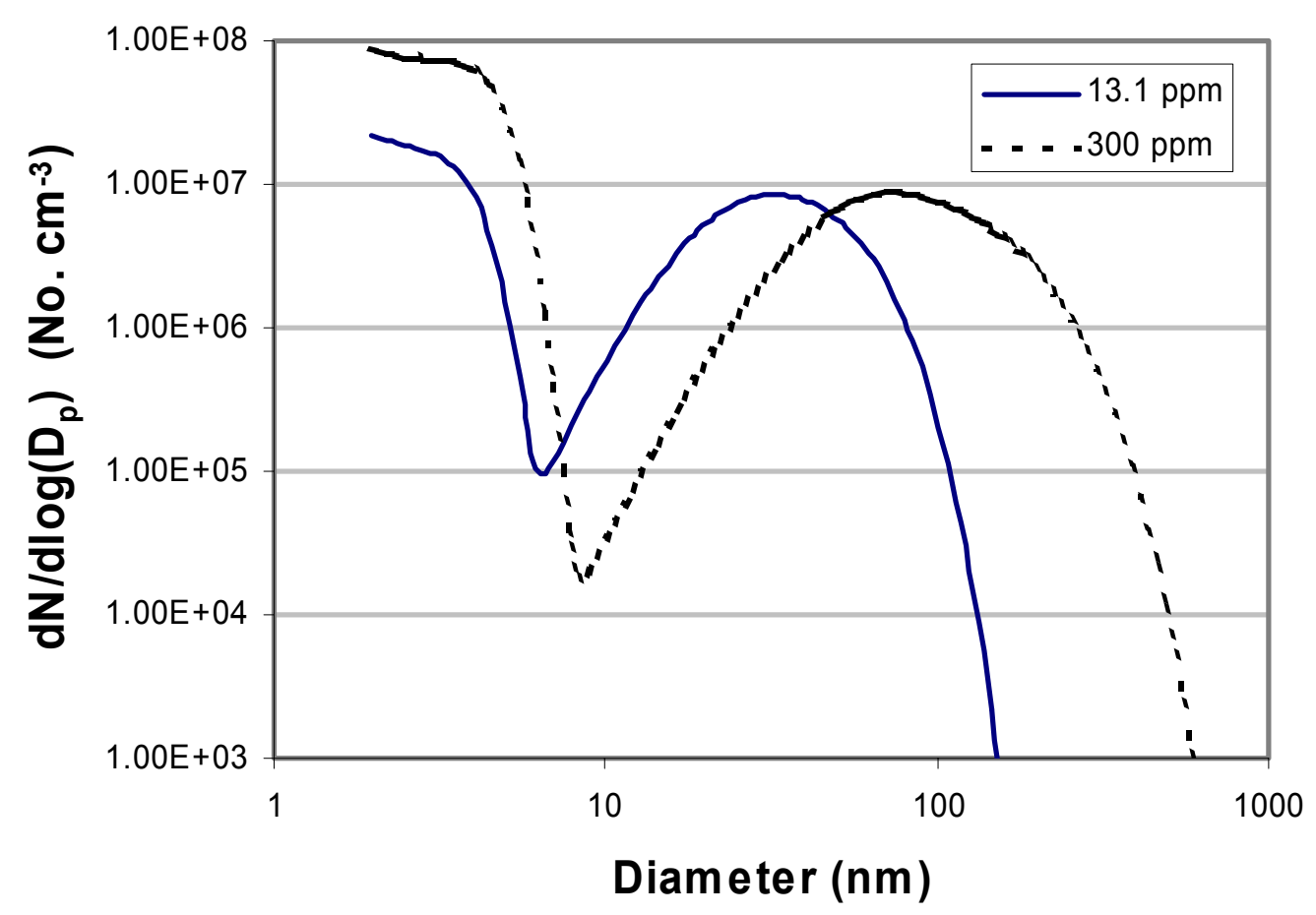

Figure 4.48 Model prediction on particle number concentration of different sulfur levels $(13.1 \mathrm{ppm}$ and $300 \mathrm{ppm})$ at $0.14 \mathrm{sec}(30 \%$ relative humidity) 


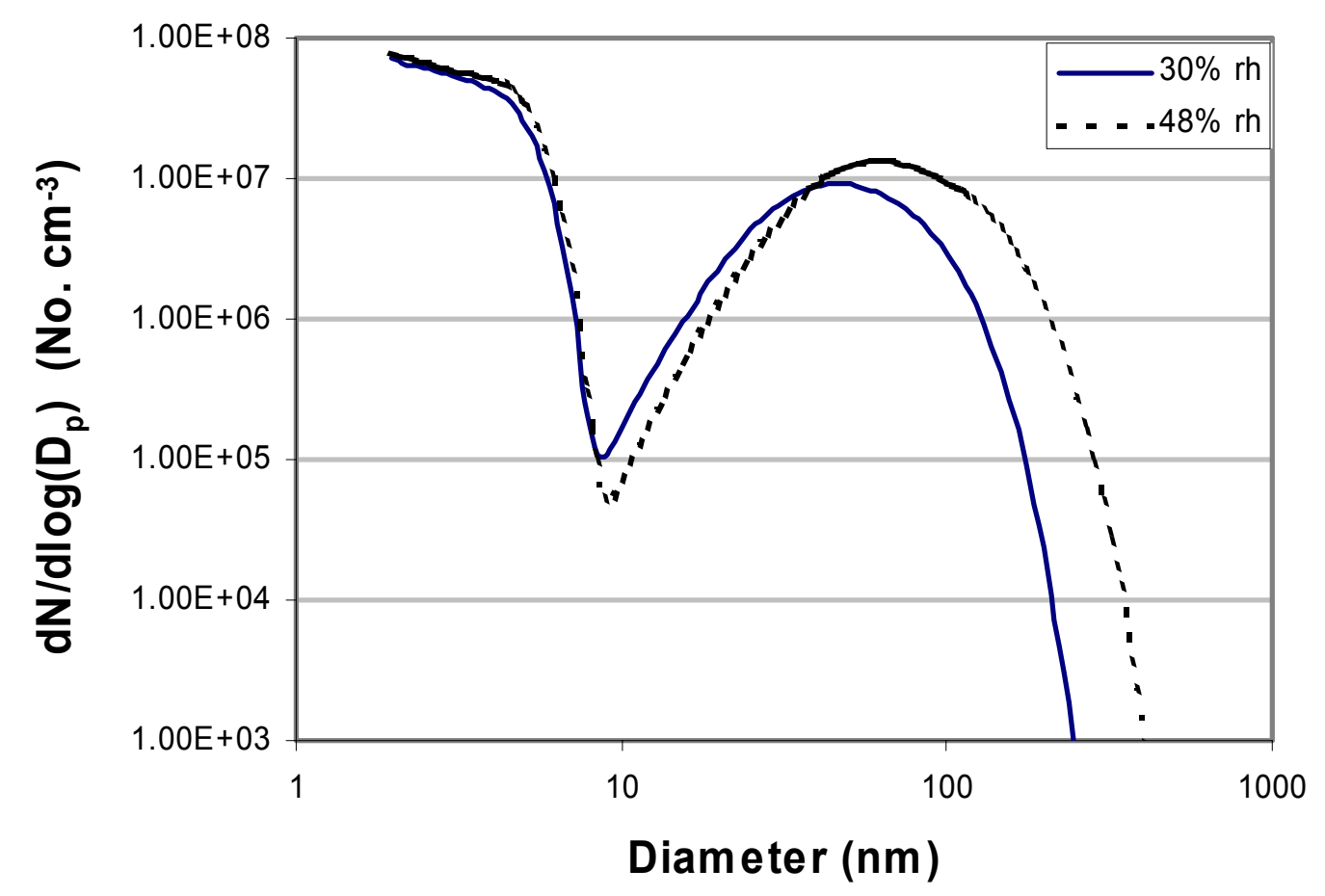

Figure 4.49 Model prediction on particle number concentration of different relative humidity on $125 \mathrm{ppm}$ sulfur fuel content at $0.17 \mathrm{sec}$ 
The summary of the major findings of this research is presented in table 4.1.

Table 4.1 Summary of the major findings of the current research

\begin{tabular}{|c|c|}
\hline Processes & Summary \\
\hline $\begin{array}{l}\text { FLUENT k- } \varepsilon \text { / } \\
\text { Finite Rate } \\
\text { Chemistry } \\
\text { Model }\end{array}$ & $\begin{array}{l}\text { The CFD results in Figure } 4.4 \text { predicted that the center of plume was } \\
\text { pointing slightly downward, which may be attributed to the wake effects } \\
\text { behind the truck. The relative concentration of } \mathrm{CO}_{2} \text { calculated from the } \\
\text { finite rate chemistry model in Figure } 4.5 \text { depicted a rapid drop from } 1 \text { to } \\
0.01 \text { within } 2.54 \mathrm{~m} \text { (100") downstream of the exhaust stack outlet. } \\
\text { Velocity vectors predicted from k- } \varepsilon \text { model in Figure } 4.6 \text { showed a } \\
\text { significant recirculation of the flow below the wind deflector. In the } \\
\text { absence of any physical obstacle above the centerline, the plume } \\
\text { dispersed very quickly. The temperature predicted from the CFD model } \\
\text { in Figure } 4.9 \text { also illustrated a similar rapid drop as the relative } \\
\text { concentration to the ambient value within } 2.54 \mathrm{~m}(100 \text { ") downstream of } \\
\text { the exhaust stack outlet }\end{array}$ \\
\hline Gaussian/PDF & $\begin{array}{l}\text { In recirculation region below the centerline of plume, the Gaussian } \\
\text { model was modified by Equations (3.14-15). The dispersion coefficients } \\
\text { were determined using the experimental data to calibrate the Gaussian } \\
\text { for the present configuration. The above CFD model was extended to } \\
\text { model the species transport using the probability density function (PDF) } \\
\text { formulation in Section } 4.2 .2 \text {. The } \mathrm{CO}_{2} \text { concentration prediction from the } \\
\text { Gaussian and PDF model agreed well with the experimental data. }\end{array}$ \\
\hline Condensation & $\begin{array}{l}\text { Homogeneous nucleation created a large numbers of new particles, } \\
\text { and it was highly nonlinear with the saturation ratio- evidenced from } \\
\text { Figures ( } 4.18-4.26) \text {. Nucleation increased number concentration/volume } \\
\text { concentration with increasing relative humidity but the nucleus diameter } \\
\text { decreased with increasing relative humidity. The nucleation rate } \\
\text { predicted in Figure } 4.15 \text { dropped by } 4 \text { orders of magnitude with the } \\
21^{\circ} \text { C increase in the ambient temperature. } \\
\text { Coagulation process was strongly dependent on initial particle size } \\
\text { and concentration. Coagulation caused a decrease in particle number } \\
\text { concentration and increase in particle size as shown in Figures } 4.36- \\
4.39 \text {. Coagulation/dispersion rate of the PM predicted by Equation (4.4) } \\
\text { decreased with the increase in viscosity. } \\
\text { Figures } 4.40 \text { predicted that the condensation effects were very } \\
\text { important near the stack outlet, where rapid dilution of exhaust was } \\
\text { taking place with the ambient air. The CMD predicted from the current } \\
\text { model shifted from } 52 \text { nm to } 10 \text { nm if the condensation effects were } \\
\text { neglected. Figures } 4.40 \text { and } 4.41 \text { predicted an increase in CMD from } 52 \\
\text { nm at } 0.51 \text { m ( } 20 \text { ") from the stack exit to } 62 \text { nm at a distance of } 8.56 \mathrm{~m} \\
\text { (337') from the stack exit, and the number concentration decreased from } \\
8.77 \text { E+ } 6 \text { to } 2.1 \text { E }+5 \text { No./cm }{ }^{3} \text {. }\end{array}$ \\
\hline
\end{tabular}




\section{CHAPTER 5}

\section{CONCLUSIONS AND FUTURE RECOMMENDATIONS}

This effort used the state-of-the-art computational fluid dynamics (CFD) concepts and tools to model the dispersion of a turbulent plume emitted by a heavy-duty tractor truck traveling at $55 \mathrm{mph}$ on a highway. A commercial CFD software FLUENT ${ }^{\mathrm{TM}}$ was employed for accurately formulating the physical phenomena important to the plume dispersion model that included the effects of turbulent mixing, convection, diffusion, temperature variations, and species transport among others. The relative concentration of $\mathrm{CO}_{2}$, dilution ratio, and temperature variations inside the plume predicted by the CFD model were validated with the experimental data obtained from controlled wind tunnel tests. It was predicted from this finite rate chemistry eddy dissipation model that the relative concentration of $\mathrm{CO}_{2}$ dropped rapidly from 1 to approximately 0.01 , and the dilution ratio increased from 1 to 100 within a distance of $100^{\prime \prime}(2.54 \mathrm{~m})$ downstream of the exhaust stack outlet. This is due to the fact that the small flow rate coming out of the exhaust pipe is turbulently mixing with the ambient air. The CFD simulations predicted the center of the plume to be pointing slightly downwards, which was due to the wake effects behind the truck. Velocity vectors predicted from the numerical model showed a significant recirculation of the flow below the wind deflector, which was extended to 65 " $(1.65 \mathrm{~m})$ from the back wall of the cabin. This was because in the CFD model, the dispersion coefficients were not assumed constant, unlike in traditional Gaussian models, but they included the effect of turbulent mixing. It was concluded that the CFD models could be used to predict the dispersion of pollutants accurately, and to evaluate the impact of emission of pollutants on the environment. 
Additionally, the relative concentration of $\mathrm{CO}_{2}$ variation inside the plume was predicted using the modified Gaussian model and the probability density function (PDF) mixture fraction formulation. Gaussian model was modified in this study to account for the re-circulation region (below the centerline of plume) behind the truck. In the Gaussian model, experimental data was needed to find the dispersion coefficients, and it was calibrated for this configuration. The maximum error in predicting the relative concentration of $\mathrm{CO}_{2}$ and the dilution ratio from the PDF model was less than $10 \%$ of the values predicted from the finite rate chemistry eddy dissipation model. In the PDF formulation, transport of mean mixture fraction and its variance were solved instead of solving the transport equations for every species.

The nucleation rates in the formation of PM were calculated directly from the sulfur content in the fuel, and hydration effects were included in the nucleation submodel. It was inferred that the critical nucleus diameter decreased by approximately $30 \%$ and the number concentration increased by a factor of 6 with the increase in relative humidity from $10 \%$ to $90 \%$ for a fuel with 50 ppm Sulfur content. The similar trends were also predicted for the fuels with $13.1 \mathrm{ppm}, 125 \mathrm{ppm}$, and $300 \mathrm{ppm}$ sulfur content. It was inferred that the condensation effects were very important near the stack outlet where the rapid dilution of particulate matter with the ambient air was dominant. It was predicted that if the contribution from the condensation term was included then the PM count median diameter increased from approximately $10 \mathrm{~nm}$ to $52 \mathrm{~nm}$. A good agreement was seen between the predicted PM concentration values and the PM concentrations 
measured at four different locations in a turbulent plume from the diesel exhaust in the wind tunnel. The root mean square (RMS) error in the predicted particle number concentration with respect to the experimentally measured values was $14.3 \%$.

The effects of ambient dilution and viscosity on particulate matter (PM) size distribution and concentration variation were presented and discussed. It was inferred that the coagulation/dispersion of PM decreased with increasing viscosity. It was predicted that the count median diameter (CMD) increased from $52 \mathrm{~nm}$ to $1000 \mathrm{~nm}$ when the ambient viscosity changed from 5.79 E-2 dynes.sec $/ \mathrm{cm}^{2}\left(5.79 \mathrm{E}-3 \mathrm{~N} . \mathrm{sec} / \mathrm{m}^{2}\right)$ to $1.79 \mathrm{E}-4$ dynes.sec $/ \mathrm{cm}^{2}\left(1.79 \mathrm{E}-5 \mathrm{~N} \cdot \mathrm{sec} / \mathrm{m}^{2}\right)$. The current numerical model predicted an increase in CMD from $52 \mathrm{~nm}$ at $0.51 \mathrm{~m}$ (20") from the stack exit to $62 \mathrm{~nm}$ at a distance of $8.56 \mathrm{~m}$ (337”) from the stack exit, and the number concentration decreased from $8.77 \mathrm{E}+6$ to 2.1 $\mathrm{E}+5 \mathrm{No} . / \mathrm{cm}^{3}$.

The current study was important because the scientific, engineering, health and regulatory bodies are now focusing not only on mass concentrations but also the number concentrations for ultra fine particulate matter. Ultra fine $(<100 \mathrm{~nm})$ particulate matter, present in very high numbers (even with insignificant percent of total mass) and large surface areas for biological interaction, can pose serious health hazards. To better understand the challenges posed by PM emissions, an accurate prediction of number density of ultra fine particles is necessary, and has been presented in this study. 


\subsection{FUTURE RECOMMENDATIONS}

- The particulate matter measurements should be made, if possible, within $2 "(0.05 \mathrm{~m})$ from the stack pipe exit to quantify the exact nucleation rate. The experiments should be repeated for various sulfur concentrations in the fuel and at different relative humidity levels.

- Atomization of Fuel droplets should be modeled using the 'developing' moving mesh in-cylinder combustion modeling techniques. This will help in determining the contribution of unburned carbon from the incomplete combustion of fuel droplets on PM concentration and size distribution.

- The effect of heavy hydrocarbons on the nucleation rate should be investigated. It is envisaged that the heavy hydrocarbon will initiate the heterogeneous nucleation.

- The tests should be repeated for some old technology ( $\sim 15$ years old) and new technology engines to investigate the effects of various atomizers on nucleation rates. This will also determine if the valve opening/closing times have a direct impact on particulate matter formation.

- It was inferred that the apparent viscosity of the ambient media played a role in the coagulation of particles. To confirm this postulate, some tests may be conducted at different operational speeds and loads on the truck.

- Nucleation and coagulation modules may be grouped with the commercial code to couple the aerodynamics and particle formation.

- Another test may be conducted for the stationary trucks in relatively calm ambient air to analyze the vertical plumes. This test will mimic the conditions when the truck is 
standing at a traffic light in a city. After learning from this configuration, the current model can then be extended to the formation of particles from the stacks of power plants and high temperature furnaces.

- The current models may be extended to other engines, for example gasoline and compressed natural gas engines. 


\section{REFERENCES}

Abdul-Khalek, I. S., Kittleson, D. B., Graskow, B. R., Wei, Q., and Brear, F. (1998): "Diesel Exhaust Particle Size: Measurement Issues and Trends", Society of Automobile Engineers, SAE 980525

Ackermann, I. J., Hass, H., Memmesheimer, M., Ebel, A., Binkowski, F. S. and Shankar, U. (1998): "Modal Aerosol Dynamics Model for Europe: Development and First Applications", Atmospheric Environment, Vol. 32, pp. 2981-2999

Ahlvik, P., Ntxiachristos, L., Keskinen, J., and Virtanen, A. (1998): "Real Time Measurements of Diesel Particle Size Distribution with an Electrical Low Pressure Impactor", Society of Automobile Engineers, SAE 980410

Ansari, A. S., and Pandis, S. N. (1998): "Response of Inorganic PM to Precursor Concentrations", Environmental Science and Technology, Vol. 32, pp. 2706-2714

Ansari, A. S., and Pandis, S. N.(1999): "Prediction of Multicomponent Inorganic Atmospheric Aerosol Behavior", Atmospheric Environment, Vol. 33, pp. 745-757

Ayers, G. P., Gillett, R. W., and Gras, J. L. (1980): "On the Vapor Pressure of Sulfuric Acid", Geophysical Research Letters, Vol. 7, pp. 433-436

Bagley, S. T., Gratiz, L. D., Johnson, J. H. and McDonald, J. F. (1998): "Effects of an Oxidation Catalytic Converter and a Biodiesel Fuel on the Chemical, Mutagenic, and Particle Size Characteristics of Emissions from a Diesel Engine", Environmental science and technology, Vol. 32, pp. 1183-1191

Barrett, J. C. (1992): "Nucleation with Changing Saturation", Journal of Aerosol Science, Vol. 23, pp. S141-S144

Bassett, M. and Seinfeld, J. H. (1983): "Atmospheric Equilibrium Model of Sulfate and Nitrate Aerosols", Atmospheric Environment, Vol. 17, pp.2237-2252

Bassett, M. E. and Seinfeld, J. H. (1984): "Atmospheric Equilibrium Model of Sulfate and Nitrate Aerosols-II.Particle Size Analysis", Atmospheric Environment, Vol. 18, pp. 1163-1170

Baumgard, K. J., and Johnson, J. H. (1992): "The Effect of Low Sulfur Fuel and a Ceramic Particle Filter on Diesel Exhaust Particle Size Distributions", SAE Tran., Vol. 101, pp.691-699

Baumgard, K. J., and Johnson, J. H. (1996): "The Effect of Fuel and Engine Design on Diesel Exhaust Particle Size Distributions", Society of Automobile Engineers, SAE 960131 paper, pp. 37-50

Bellasio, R. and Tamponi, M. (1994): "MDGP: a New Eulerian 3D Unsteady State Model for Heavy Gas Dispersion", Atmospheric Environment, Vol. 28, pp.16331643

Bischof, O. F. (1998): "The SMPS, the Most Widely Used Nanoparticle Sizer", Second International ETH Workshop on Nanoparticle Measurement, Zurich, Switzerland, August 1998

Black, F., Tejada, S. and Kleindienst, T. (1998): "Preparation of Automobile Organic Emission Surrogates for Photochemical Model Validation", Atmospheric Environment, Vol. 32, pp. 2443-2451 
Bowman, F. M., Odum, J. R., Seinfeld, J. H. and Pandis, S. N. (1997): "Mathematical Model for Gas-Particle Partitioning of Secondary Organic Aerosols", Atmospheric Environment, Vol. 31, pp. 3921-3931

Boyce, J., Mehta, S., Gautam, M., and Clark, N. N. (2000): "Heavy Duty Diesel Truck Research in the ODU/Langley Wind Tunnel (CRC E-43)", $10^{\text {th }}$ CRC On-Road Vehicle Emissions Workshop, San Diego, California, March 27-29, 2000

Brocco, A. L., and Possanzini, M. (1978): "Adsorption and Oxidation of Sulfur Dioxide on Particles", Atmospheric Environment, Vol. 12, pp. 255-261

Brown , G. W. (1956): Monte Carlo methods, Modern Mathematics for the Engineer (Ed. E.F.Beckenbach), McGraw-Hill, New York, pp. 279-303

Brown, J. E., Calyton, M. J., Harris, D. B., and King, F. G.Jr., (2000): "Comparison of the Particle Size Distribution of Heavy-Duty Diesel Exhaust Using a Dilution Tailpipe Sampler and an In-Plume Sampler During On-Road Operation", Journal of Air \& Waste Management Association, Vol. 50, pp. 1407-1416

Brown, R. C., Miake-Lye, R. C., Anderson, M. R., Kolb, C. E., and Resch, T. J. (1996): "Aerosol Dynamics in Near-Field Aircraft Plumes", Jounral of Geophysical Research, Vol. 101, No. D17, pp. 22,939-22,953

Bugarski, A. D. (1999): Characterization of Particulate Matter and Hydrocarbon Emissions from In-Use Heavy-Duty Diesel Engines, Ph.D. Dissertation, Mechanical and Aerospace Engineering, West Virginia University, Morgantown, WV

Cadle, S. H. (1985): "Seasonal Variations in Nitric Acid, Nitrate, Strong Aerosol Acidity, and Ammonia in an Urban Area", Atmospheric Environment, Vol. 19, pp. 181188

Caritat, P. D., Reimann, C., Chekushin, V., Bogatyrev, I., Niskavaara, H. and Braun, J. (1997): "Mass Balance Between Emission and Deposition of Airbone Contaminants", Environmental Science and Technology, Vol. 31, pp. 2966-2972

Carmichael, G. R. and Peter, L. K. (1984): "An Eulerian Transport/ Transformation/Removal Model for $\mathrm{SO}_{2}$ and Sulate-1.Model Development", Atmospheric Environment, Vol. 18, pp. 937-951

Carter,W. P. L.(1990): "A Detailed Mechanism for the Gas-Phase Atmospheric Reactions of Organic Compounds", Atmospheric Environment, Vol. 24A, pp. 481-518

Chemical Engineer's Handbook (1973) Perry, R. H., and Chilton, C. H., Editions, McGraw-Hill, New York

Chrysikopoulous, C. V., Hildemann, L. M. and Roberts, P. V. (1992): “A ThreeDimensional Steady-State Atmospheric Dispersion-Deposition Model for Emissions from a Ground-Level Area Source", Atmospheric Environment, Vol. 26A, pp. 747-757

Clement, C. F. and Ford, I. J. (1999): "Gas-to-Particle Conversion in the Atmosphere: I. Evidence from Empirical Atmospheric Aerosols", Atmospheric Environment, Vol. 33, pp. 475-487

Clement, C. F. and Ford, I. J. (1999): "Gas-to-Particle Conversion in the Atmosphere: II. Analytical Models of Nucleation Bursts", Atmospheric Environment, Vol. 33, pp. 489-499

Costantitni, M. G. (2000): "Relation Between Particle Metal Content (With Focus on Iron) and Biological Responses," $4{ }^{\mathrm{TH}}$ International ETH Conference on Nanoparticle Measurement, Vol. 1, Zurich, August 7-9, 2000 
Csanady, G. T (1967): "Concentration Fluctuations in Turbulent Diffusion", Journal of Atmospheric Science, Vol. 24, pp. 21-28

De Nerves, N. (1995): Air Pollution Control Engineering, McGraw-Hill, Inc., 506 pp.

Derksen, R.W., and Kleiman, M. (1988): "Characterization of Concentration Fluctuations of a Surface Plume in a Neutral Boundary Layer," Boundary-Layer Met., Vol. 45, p. 378-391

Dhaniyala, S. and Wexler, A. S. (1996): "Numerical Schemes to Model Condensation and Evaporation of Aerosols", Atmospheric Environment, Vol. 30, pp. 919928

Dingene, R. V. and Raes, F. (1990): "Coagulation Enhancement of $\mathrm{H}_{2} \mathrm{SO}_{4}-\mathrm{H}_{2} \mathrm{O}$ Aerosols: Experiments and Model Calculations in the Transition Regime", Journal of Aerosol Science, Vol. 21, Suppl. 1, pp. S237-S240

Dockery, D. W., Pope, C. A., Xu, X, Spengler, J. D., Ware, J. H., Fay, M. E., Ferris, B. G., and Seizer, F. E. (1993): "An Association Between Air Pollution and Mortality in Six U.S. Cities", Massachusetts Medical Society Journal of Medicine, Vol. 329, No. 24, pp. 1753-1759

Donaldson, K., Beswich, P. H., and Gilmour, P. S. (1996): "Free Radical Activity Associated with the Surface of Particles: a Unifying Factor in Determining Biological Activity?", Toxicology Letter, Vol. 8, pp. 293-298

Donaldson, K., Li, X. Y., MacNee, W. (1998): "Ultrafine (Nanometer) ParticleMediated Lung Injury”, Journal of Aerosol Science, Vol. 29, pp. 553-560

Doyle G. J. (1961): "Self-Uncleation in the Sulfuric Acid-Water System", Journal of chemical physics, Vol. 35, No. 3, pp. 795-799

Durbin, P. A. (1980): "A Statistic Model Of Two Particle Dispersion And Concentration Fluctuations In Homogeneous Turbulence", Journal of Fluid Mechanics, Vol. 100, No. 2, pp. 279-302

Enukashivili, I. M. (1964): "On the Problem of a Kinetic Theory of Gravitational Coagulation in Spatially Heterogeneous Clouds", Izv. Geophs. Ser., No. 11, pp. 1043-5 (Trans by J.S. Sweet)

Ferin, J., Oberdorster, G., and Penney, D. P. (1992): "Pulmonary Retention of Ultrafine and Fine Particles in Rats", American Journal of Respiratory Cell Molecular Biology, Vol. 6, pp. 535-542

Ferrero, E. and Anfossi, D. (1998): "Comparison Of PDFs, Closure Schemes And Turbulence Parameterizations In Lagrangian Stochastic Models", International Journal of Environment and Pollution, Vol. 9, No. 4, pp 384-410

Flood, H. (1934): “Tröpfenbidung in Ubersättigten AthylalkoholWasserdampfgemischen", Z. Phys. Chem., Vol. A170, 286-294

Frenzel, F., and Arnold, F. (1994): "Sulfuric Acid Cluster Ion Formation by Jet Engines: Implications for Sulfuric Acid Formation and Nucleation", Report. DLR Mitt, 94-06, Dtsch. Forsch. Fur Luft- und Raumfahrt, Koln, Germany

Friedlander, S. K. and Wang, C. S. (1966): "The Self-Preserving Particle Size Distribution for Coagulation by Brownian Motion", Journal of Colloid Interface Science, Vol. 22,pp. 126-32

Friedlander, S. K. (1977): Smoke, Dust and Haze: Fundamental of Aerosol Behavior, Wiley, New York 
Fuchs, N (1964): The Mechanics of Aerosols, A Pergamon Press Book, The Macmillan Company, 60 Fifth Ave, New York, NY

Gautam, M. and Mehta, S. (2001): "Private Communications", Mechanical and Aerospace Engineering, West Virginia University, Morgantown

Gautam, M., Xu, Z., Ayala, A., and Mehta, S. (2000): "Diesel Exhaust Plume Studies: Wind Tunnel Experiments and Modeling", Fourth ETH Nanoparticle Measurement Workshop, August 7 - 9, 2000, Zurich

Giauque, W. F., Hornung, E. W., Kunzler, J. E., and Rubin, T. R. (1960): “The Thermodynamic Properties of Aqueous Sulfuric Acid Solutions and Hydrates from 15 to 300 'k", Journal of American Chemical Society, Vol. 82, pp. 62-70

Gifford, F. A. (1959): "Statistical Properties Of A Plume Dispersion Model", Advance Geophysics, Vol. 6, pp. 117-138

Gifford, F. A. (1961): "Use of Routine Meteorological Observations for Estimating Atmospheric Dispersion”, Nucl. Safety, Vol. 2, pp. 47-51

Gifford, F. A. (1982): "Horizontal Diffusion In The Atmosphere: A LagrangianDynamical Theory", Atmospheric Environment, Vol. 16, pp. 505-512

Golovin, A. M. (1963): "On the Spectrum of Coagulating Cloud Droplets", II, Izv. Geophys. Ser., No. 9, pp. 880-4 (trans by C.M. Wade)

Graskow, B. R., Kittelson, D. B., Abdul-Khalek, I. S., Ahmadi, M. R. and Morris, J. E. (1998): "Characterization of Exhaust Particulate Emissions from a Spark Ignition Engine", Society of Automobile Engineers, SAE paper 980528, pp. 155-165

Gray, H. A. and Cass, G. R. (1998): "Source Contributions to Atmospheric Fine Carbon Particle Concentrations", Atmospheric Environment, Vol. 32, pp. 3805-3825

Hall, R. J. and Colket, M. B. (1992): "Predictions of Soot Particle Growth Based on Aerosol Dynamics Modeling", Journal of Aerosol Science, Vol. 23, pp. S129-132

Hanna, S. R. (1984): "Concentration Fluctuations In A Smoke Plume", Atmospheric Environment, Vol. 18, No. 6, pp. 1091-1106

Heinz, S., and van Dop, H. (1999): "Buoyant Plume Rise Described By A Lagrangian Turbulence Model," Atmospheric Environment, Vol. 33, No. 13, pp. 20312043

Publishing Co.

Heywood, J. B. (1988): Internal Combustion Engine Fundamentals, McGraw-Hill

Hidy, G. M. and Brock, J. R. (1972): Topics in Current Aerosol Research, Pergamon Press Ltd., Headington Hill Hall, Oxford, England

Hildemann, L. M., Russell, A. G. and Cass, G. R. (1984): “Ammonia and Nitric Acid Concentrations in Equilibrium with Atmospheric Aerosols: Experiment vs Theory", Atmospheric Environment, Vol. 18, pp. 1737-1750

Hilst, G. R. (1998): "Segregation and Chemical Reaction Rates in Air Quality Models", Atmospheric Environment, Vol. 32, pp. 3891-3895

Hinds, W. (1982): Aerosol Technology-Properties, Behavior, and Measurement of Airborne Particles, John Wiley and Sons, Inc. pp. 424

Hov, O. (1983): 'Numerical Solution of a Simplified form of the Diffusion Equation for Chemical Reactive Atmospheric Species", Atmospheric Environment, Vol. 17 , pp. 551-562 
Hov, O., Zlatev, Z., Berkowicz, R., Eliassen, A. and Prahm, L. P. (1989): "Comparison of Numerical Techniques for Use in Air Pollution Models with Non-Linear Chemical Reactions", Atmospheric Environment, Vol. 23, pp. 967-983

Huai, W. and Li, W. (1993): "Similarity Solutions Of Round Jets And Plumes," Applied Mathematics and Mechanics (English Edition), Vol. 14, No. 7, pp 649-658

Huber, A. H. (1991): "Wind Tunnel and Gaussian Plume Modeling of Building Wake Dispersion," Atmospheric Environment, Vol. 25A, pp. 1237-1249

Hughes, L. S., Cass, G. R., Gone, J., Ames, M. and Olmez, I. (1998): "Physical and Chemical Characterization of Atmospheric Ultrafine Particles in the Los Angeles Area", Environmental science and technology, Vol. 32, pp. 1153-1161

Husar, R. (1970): Discussion presented at the Particulate Workshop held at NCAR on August 21

Hwang, R. R. and Chiang, T. P. (1988): "Numerical Simulation On Turbulent Buoyant Jets In A Flowing Stratified Ambient", Proceedings of the ASME International Mechanical Engineering Congress and Exposition, Nov 15-20 1998, Vol. 247, pp. 85-88

Jacker-Voirol, A., Mirabel, P., and Reiss, H. (1987): "Hydrates in Supersaturated Binary Sulfuric Acid-Water Vapor: a Reexamination", Journal of Chemical Physics, Vol. 87, pp. 4849-4852

Jacobson, M. Z. (1997): 'Development and Application of a New Air Pollution Modeling System-Part III: Aerosol-Phase Simulations", Atmospheric Environment, Vol. $31(4)$, pp. 587-608

Jacobson, M. Z. (1997): "Development and Application of a New Air Pollution Modeling System-Part II: Aerosol Module Structure and Design", Atmospheric Environment, Vol. 31 (2), pp.131-144

Jacobson, M. Z. and Turco, R. P. (1994): "SMVGEAR: a Sparse-Matrix, Vectorized Gear Code for Atmospheric Models", Atmospheric Environment, Vol. 28 (2), pp. 273-284

Jacobson, M. Z. and Turco, R. P. (1995): "Simulating Condensational Growth, Evaporation, and Coagulation of Aerosols Using a Combined Moving and Stationary Size Grid", Aerosol Science and Technology, Vol. 22, pp. 73-92

Jacobson, M. Z., Lu, R., Turco, R. P., and Toon, O. (1996): "Development and Application of a New Air Pollution Modeling System-Part I: Gas-Phase Simulations",Atmospheric Environment, Vol. 30 (12), pp.1939-1963

Jacobson, M. Z., Turco, R. P., Jensen, E. J. and Toon, O. B. (1994): "Modeling Coagulation among Particles of Different Composition and Size", Atmospheric Environment, Vol. 28, pp. 1327-1338

Jaecker-Voirol, A. and Mirabel, P. (1989): "Heteromolecular Nucleation in the Sulfuric Acid-Water System”, Atmospheric Environment, Vol. 23, pp. 2053-2057

John, C., Friedrich, R., Staehelin, J., Schlapfer, K. and Stahel, W. A. (1999): "Comparison of Emission Factors for Road Traffic from a Tunnel Study (Gubrist tunnel, Switzerland) and from Emission Modeling", Atmospheric Environment, Vol. 33, pp. 3367-3376

Jones, A., Roberts, D. L., and Singo, A. (1994): “A Climate Model Study of Indirect Radiative Forcing by Anthropogenic Sulphate Aerosols", Nature, Vol. 370, pp. $450-453$ 
Judge, C. (1964): "The Modification of Aerosol Size Distribution in the Atmosphere", Final Technical Report, U.S. Department of Army Contract No. Da 91591-EVC 2979, Mainz, Germany.

Kaharabata, S. K., Schuepp, P. H., and Desjardins, R. L. (2000): "Source Strength Determination Of A Tracer Gas Using An Approximate Solution To The AdvectionDiffusion Equation For Microplots", Atmospheric Environment, Vol. 34, pp. 2343-2350

Karcher, B. (1996): "Aircraft-generated Aerosols and Visible Contrails", Geophysical Research Letters, Vol. 23, No. 15, pp. 1933-1936

Katoshevski, D. and Seinfeld, J. H. (1997): “Analytical-Numerical Solution of the Multicomponent Aerosol General Dynamic Equation-with Coagulation", Aerosol Science and Technology, Vol. 27, pp.550-556

Kerminen, V-.M., Makela, T. E., Ojanen, C. H., Hillamo, R. E., Vilhunen, J. K., Rantanen, L., Havers, N., Bohlen, A. V. and Klockow, D. (1997): "Characterization of the Particulate Phase in the Exhaust from a Diesel Car", Environmental Science and Technology, Vol. 31, pp. 1883-1889

Kerminen, V-M., Viisanen, Y., Vesala, T. and Hillamo, R. (1991): "Correction for the Brownian Coagulation Coefficient due to Van der Waals Forces between NonEqual Sized Particles", Journal of Aerosol Science, Vol. 22, Suppl. 1, pp. S105-S107

Kim, D., Gautam, M., and Gera, D.(2001): "On the Prediction of Concentration Variations in a Dispersing Heavy-Duty Truck Exhaust Plume Using K- $\varepsilon$ Turbulent Closure", Atmospheric Environment, Vol. 35(31), pp. 5267-75

Kim, D., Gautam, M., and Gera, D.(2002): "Modeling Nucleation and Coagulation Modes in the Formation of Particulate Matter Inside a Turbulent Exhaust Plume of a Diesel Engine", J. Colloid and Interface Science, Vol. 249 (1), pp. 96-103

Kim, Y. P. and Seinfeld, J. H. (1995): "Atmospheric Gas-Aerosol Equilibrium: III. Thermodynamics of Crustal Elements $\mathrm{Ca}^{2+}, \mathrm{K}^{+}$, and $\mathrm{Mg}^{2+}$, Aerosol Science and Technology, Vol. 22, pp. 93-110

Kim, Y. P., Seinfeld, J. H. and Saxena, P. (1993): “Atmospheric Gas-Aerosol Equilibrium I. Thermodynamic Model", Aerosol Science and Technology, Vol. 19, pp. 157-181

Kim, Y. P., Seinfeld, J. H., and Saxena, P. (1993): “Atmospheric Gas-Aerosol Equilibrium II. Analysis of Common Approximations and Activity Coefficient Calculation Methods", Aerosol Science and Technology, Vol. 19, pp. 182-198

Kirchstetter, T. W., Harley, R. A., Kreisberg, N. M., Stolzenburgh, M. R. and Hering, S. V. (1999): "On-Road Measurement of Fine Particle and Nitrogen Oxide Emissions from Light- and Heavy-Duty Motor Vehicles", Atmospheric Environment, Vol. 33, pp. 2955-2968

Kittelson, D. B., and Johnson, J. H. (1991): "Variability in Particle Emission Measurements in the Heavy Duty Transient Test", Society of Automobile Engineers, SAE 910738

Kittelson, D. B. (1998): "Engines and Nanoparticles: a Review", Journal of Aerosol Science, Vol. 29, pp. 575-588

Kittelson, D. B., Dolan, D. E., and Verrant, J. A. (1978): "Investigation of a Diesel Exhaust Aerosol", Society of Automobile Engineers, SAE 780109 
Knutson, E. O. and Whitby, K. T. (1975): "Aerosol Classification by Electric Mobility: Apparatus, Theory, and Applications", Journal of Aerosol Science, Vol. 6, pp. 343

Kulmala, M. and Viisanen, Y. (1988): "Nucleation in Acid-Water Systems, Experimental and Theoretical Results", Journal of Aerosol Science, Vol. 19, pp. 825-828

Kulmala, M. and Viisanen, Y. (1991): "Homogeneous Nucleation: Reduction of Binary Nucleation to Homomolecular Nucleation", Journal of Aerosol Science, Vol. 22, Suppl. 1, pp. S97-S100

Kulmala, M., Kerminen, V-. M. and Laaksonen, A. (1995): "Simulations on the Effect of Sulphuric Acid Formation on Atmospheric Aerosol Concentrations", Atmospheric Environment, Vol. 29, pp. 377-382

Kulmala, M., Laaksonen, A. and Girshick, S. L. (1992): "The Self-Consistency Correction to Homogeneous Nucleation: Extension to Binary Systems", Journal of Aerosol Science, Vol. 23 (3), pp. 309-312

Kulmala, M., Laaksonene, A. and Girshick, S. L. (1992): "The Self-Consistency Correction to Homogeneous Nucleation: Extension to Binary System", Journal of Aerosol Science, Vol. 23, pp. 309-312

Kulmala, M., Laaksonene, A., and Pirjola, L. (1998): "Parameterizations for Sulfuric Acid/Water Nucleation rates", Journal of Geophysical Research, Vol. 103, pp. 8301-8307

Kulmala, M., Laaksonene, A., Vesala, T., Korhonen, P. and Barrett, J. C. (1992): "Activation and Growth of Cloud Condensation Nuclei by Binary Nucleation and Condensation Processes", Journal of Aerosol Science, Vol. 23, pp. S113-S116

Kulmala, M., Vesala, T., and Wagner, P. E. (1992): “An Analytical Expression for the Rate of Binary Condensational Particle Growth: Comparison with Numerical Results", Journal of Aerosol Science, Vol. 23, pp. S133-S136

Laaksonen, A. and Kulmala, M. (1991): "Homogeneous Heteromolecular Nucleation of Sulfuric Acid and Water Vapours in Stratospheric Conditions: a Theoretical Study of the Effect of Hydrate Interaction", Journal of Aerosol Science, Vol. 22, pp. 779-787

Lamb, R. C. (1982): Diffusion in the Convective Boundary Layer, In Atmospheric Turbulence and Air Pollution Modelling, (edited by Nieuwstadt F.T.M. and VonDop H.), pp. 159-229, D. Reidel, Dordrecht, Holland.

Lamb, R.C. (1982): "Diffusion In The Convective Boundary Layer", In Atmospheric Turbulence and Air Pollution Modeling, (edited by Nieuwstadt F.T.M. and VonDop H.), pp. 159-229, D. Reidel, Dordrecht, Holland.

Lazaridis, M., Kulmala, M. and Gorbunov, B. Z. (1992): "Binary Heterogeneous Nucleation at a Non-Uniform Surface”, Journal of Aerosol Science, Vol. 23, pp. 467-466

Lesnic, D., Elliott, L. and Ingham, D. B. (1996): "A Numerical Analysis of the Data Inversion of Particle Sizing Instruments", Journal of Aerosol Science, Vol.27, pp. 1063-1082

Lewellen, W.S. and Sykes, R. I. (1986): “Analysis of Concentration Fluctuations from Lidar Observations of Atmospheric Plumes," J. of Clim. Appl. Met., Vol.85, pp. $1145-1154$ 
Lighty, J. S., Veranth, J. M., Sarofim, A. F. (2000): "Combustion Aerosols: Factors Governing Their Size and Composition and Implications to Human Health", Journal of the Air and Waste Management Association, Vol. 50, pp. 1565-1618

Lippmann, M. (1976): Size-Selective Sampling for Inhalation Hazard Evaluation. In Fine Particles: Aerosol Generation, Measurements, Sampling, and Analysis, Edited by Liu, B.Y.H., Academic Press, Orlando, FL, pp. 287-310

Luo, L., Pipho, M. J., Ambs, J. L., and Kittleson, D. B. (1989): "Particle Growth and Oxidation in a Direct-Injection Diesel Engine", Society of Automobile Engineers, SAE No. 890580

Lurmann, F. W., Wexler, A. S., Pandis, S. N., Musarra, S., Kumar, N., Seinfeld, J. H. (1997): "Modeling Urban and Regional Aerosols: II. Applications to California's South Coast Air Basin", Atmospheric Environment, Vol. 31, pp. 2695-2715

Mamane, Y., and Gottlieb, J. (1989): "The Study of Heterogeneous Reactions of Carbonaceous Particles with Sulfur and Nitrogen Oxides using a Single Particle Approach", Journal of Aerosol Science, Vol. 20, No. 5, pp. 575-584

Meng, Z., and Seinfeld, J. H. (1996): "Time Scales to Achieve Atmospheric GasAerosol Equilibrium for Volatile Species", Atmospheric Environment, Vol. 13, pp. 28892900

Meng, Z., Seinfeld, J. H., Saxena, P. (1995): “Gas/Aerosol Distribution of Formic and Acetic Acids", Aerosol Science and Technology, Vol. 23, pp. 561-578

Meng, Z., Seinfeld, J. H., Saxena, P. and Kim, Y. P. (1995): "Atmospheric GasAerosol Equilibrium: IV. Thermodynamics of Carbonates", Aerosol Science and Technology, Vol. 23, pp. 131-154

Meszaros, A. and Meszaros, E. (1989): "Sulfate Formation on Elemental Carbon Particles", Aerosol Science and Technology, Vol. 10, pp. 337-342

Middleton, P. and Kiang, C. S. (1978): "A Kinetic Aerosol Model for the Formation and Growth of Secondary Sulfuric Acid Particles", Journal of Aerosol Science, Vol. 9, pp. 359-385

Mirabel, P., and Katz, J. L. (1974): "Binary Homogeneous Nucleation as a Mechanism for the Formation of Aerosols", The Journal of Chemical Physics, Vol. 60, pp. 1138-1144

Morawska, L., Bofinger, N. D., Kocis, L. and Nwankwoala, A. (1998): "Submicrometer and Supermicrometer Particles from Diesel Vehicle Emissions", Environmental Science and Technology, Vol. 32, pp. 2033-2042

Morgan, W. K. C., Reger, R. B., Tucker, D. M. (1997): "Health Effects of Diesel Emissions", Annals of Occupational Hygiene, Vol. 41, pp. 643-658

Mostafa, K., Gautam, M., and McMillian, M. (2001): "Effect of Sulfur Content of Lubricant and Diesel Fuel on Concentration and Size Distributions of Exhaust Particulate Matter Emissions", $11^{\text {th }}$ CRC On-Road Vehicle Emissions Workshop, San Diego, CA, March 26-28

Mountain, R. D., Mulholland, G. W., and Baum, H. (1986): "Simulation of Aerosol Agglomeration in the Free Molecular and Continuum Flow Regimes", Journal of Colloid and Interface Science, Vol. 114, pp. 67-81

Muller, H. (1928): "Zur Allgemeinen Theorie der Raschen Koagulation", Kolloidchemische Beihefte, Vol. 27, pp. 223-50 
Nguyen, H. V., Okuyama, K., Mimura, T., Kousaka, Y., Flagan, R. C., and Seinfeld, J. H. (1986): "Homogeneous and Heterogeneous Nucleation in a Laminar Flow Aerosol Generator", Journal of Colloid and Interface Science, Vol. 119, pp. 491-504

Obasaju, E. D. and Robins, A. G. (1998): "Simulation Of Pollution Dispersion Using Small Scale Physical Models - An Assessment Of Scaling Options", Environmental Monitoring and Assessment, Vol. 52, No. 1-2, pp. 239-254

Pasquill, F. (1974): Atmospheric Diffusion, John Wiley, Chichester, England.

Pasquill, F., and Smith, F. B. (1983): Atmospheric Diffusion, $3^{\text {rd }}$ Ed., Wiley, NY

Pataky, G. M., Baumgard, K. J., Gratz, L. D., Bagley, S. T., Leddy, D. G., and Johnson, J. H. (1994): "Effects of an Oxidation Catalytic Converted on Regulated and Unregulated Diesel Emissions", ", Society of Automobile Engineers, SAE 940243

Peterson, T. W., Gelbard, F., and Seinfeld, J. H. (1978): "Dynamics of SourceReinforced, Coagulating and Condensing Aerosols", Journal of Colloid Interface Science, Vol. 63, pp. 426-445

Pilinis, C. (1990): "Derivation and Numerical Solution of the Species Mass Distribution Equations for Multicomponent Particulate Systems", Atmospheric Environment, Vol. 24A, pp. 1923-1928

Pilinis, C. and Seinfeld, J. H. (1987): "Continued Development of a General Equilibrium Model for Inorganic Multicomponent Atmospheric Aerosols", Atmospheric Environment, Vol. 21, pp. 2453-2366

Pilinis, C. and Seinfeld, J. H. (1988): "Development and Evaluation of an Eulerian Photochemical Gas-Aerosol Model, Atmospheric Environment", Atmospheric Environment, Vol. 22, pp. 1985-2001

Pope, C. A., Burnett, R. T., Thun, M. J., Calle, E. E., Krewski, D., Ito, K., Thurston, G. D. (2002): "Lung Cancer, Cardiopulmonary Mortality, and Long-Term Exposure to Fine Particulate Air Pollution", Journal of American Medical Association, Vol. 287,pp. 1132-1141

Raes, F., Saltelli, A. and Dingenen, R.V. (1992): "Modelling Formation and Growth of $\mathrm{H}_{2} \mathrm{SO}_{4}-\mathrm{H}_{2} \mathrm{O}$ Aerosols: Uncertainty Analysis and Experimental Evaluation", Journal of Aerosol Science, Vol. 23, pp. 759-771

Reiss, J. (1950): "The Kinetics of Phase Transition in Binary System", Journal of Chemical Physics, Vol. 18, pp. 840-848

Reynolds, A. M. (2000): “Representation Of Internal Plume Structure In Gifford's Meandering Plume Model", Atmospheric Environment, Vol. 34, pp. 2539-2545

Rickeard, D. J., Bateman, J. R., Kwon, Y. K., McAughey, J. J. and Dickens, C. J. (1996): "Exhaust Particulate Size Distribution: Vehicle and Fuel Influence in Light Duty Vehicles", SAE Papers, No. 961980

Ristovski, Z. D., Morawska, L., Hitchins, J., Thomas, S., Greenaway, C., and Gilbert, D. (2000): "Particle Emissions from Compressed Natural Gas Engines", Journal of Aerosol Science, Vol. 31, No. 4, pp. 403-412

Roedel, W. (1979): "Measurement of Sulfuric Acid Saturation Vapor Pressure: Implications for Aerosol Formation by Heteromolecular Nucleation", Journal of Aerosol Science, Vol. 10, pp. 375-386

Sabinina, L., and Terpugow, L. (1935): "Die Oberflachenspannung des Systems Schwefelsaure-Wasser", Journal of Physical Chemistry, Vol. A173, pp. 237-241 
Sawford, B. L. (1983): "The Effect Of Gaussian Particle-Pair Distribution Function Using The Statistical Theory Of Concentration Fluctuations In Homogeneous Turbulence", Q. J. Met. Soc., Vol. 190, pp. 339-354

Sawyer, R. F., and Johnson, J. H. (1995): "Diesel Emissions and Control Technology, Chapter in Diesel Exhaust: a Critical Analysis of Emissions, Exposure and Heath Effects", A Special Report of the Institute's Diesel Working Group, Health Effects Institute, pp. 65-81

Sawyer, R. F., Harley, R. A., Cadle, S. H., Norbeck, J. M., Slott, R., Bravo, H. A. (1998): "Mobile Sources Critical Review", 1998 NARSTO Assessment, Report to Coordinating Research Council, Atlanta, G.A.

Saxena, P., Hudischewskyj, A. B., and Seigneur, C. (1986): "A Comparative Study of Equilibrium Approaches to the Chemical Characterization of Secondary Aerosols", Atmospheric Environment, Vol. 20, pp. 1471-1483

Saxena, P., Seigneur, C. and Peterson, T. W. (1983): "Modeling of Multiphase Atmospheric Aerosols", Atmospheric Environment, Vol. 17, pp. 1315-1329

Seation, A., MacNee, W., Donaldson, K., and Godden, D. (1995): "Particulate Air Pollution and Acute Health Effects", The Lancet, Vol. 345, pp. 177

Seaton, A., MacNee, W., Donaldson, K., and Godden, D. (1965): "Particulate Air Pollution and Acute Health Effects", The Lancet, Vol. 345, pp. 176-178

Seigneur, C., Hudischewskyj, A. B., Seinfeld, J. H., Whitby, K. T., Whitby, E. R., Brock, J. R., and Barnes, H. M. (1986): "Simulation of Aerosol Dynamics: a Comparative Review of Mathematical Models", Aerosol Science and Technology, Vol. 5, pp. 205-222

Seinfeld, J. H. and Pandis, S. N. (1997): Atmospheric Chemistry and Physics: From Air Pollution to Climate Change, John Wiley \& Sons, Inc., 605 Third Ave, New York, NY 10158

Sharan, M. and Yadav, A. K. (1998): "Simulation Of Diffusion Experiments Under Light Wind, Stable Conditions By A Variable K-Theory Model”, Atmospheric Environment, Vol. 32, No. 20, pp 3481-3492

Shi, J. P., and Harrison, R. M.(1999): "Investigation of Ultrafine Particle Formation during Diesel Exhaust Dilution", Environmental Science and Technology, Vol. 33, pp. 3730-3736

Shi, J. P., Harrison, R. M., and Brear, F. (1998): "Ultrafine Particle Formation During Diesel Exhaust Dilution," Second International ETH Workshop on Nano Particle Measurement, ETH Zurich, August $7^{\text {th }}$

Smoluchowski, M. V. (1918): "Versuch einer Mathematischen Theories der Koagulationskinetik Kolloider Losungen”, Z. Phys. Chem., Vol. 92, pp. 144

Spalding, D. B., (1970): "Mixing And Chemical Reaction In Steady Confined Turbulent Flames", in $13^{\text {th }}$ Symp. (Int'1.) on Combustion, The Combustion Institute

Springer, G. S. (1978): "Homogeneous Nucleation", Advances in Heat Transfer, Vol. 14, pp. 281-346

Stelson, A. W. and Seinfeld, J. H. (1982): "Relative Humidity and Temperature Dependence of the Ammonium Nitrate Dissociation Constant", Atmospheric Environment, Vol. 16, pp. 983-992 
Stelson, A. W., and Seinfeld, J. H. (1982): "Relative Humidity and ph Dependence of the Vapor Pressure of Ammonium Nitrate-Nitric Acid Solutions at $25^{\circ} \mathrm{C}$ ", Atmospheric Environment, Vol. 16, pp. 993-1000

Stelson, A. W., and Seinfeld, J. H. (1982): “Thermodynamic Prediction of the Water Activity, $\mathrm{NH}_{4} \mathrm{NO}_{3}$ Dissociation Constant, Density and Refractive Index for the $\mathrm{NH}_{4} \mathrm{NO}_{3}-\left(\mathrm{NH}_{4}\right)_{2} \mathrm{SO}_{4}-\mathrm{H}_{2} \mathrm{O}$ System at $25^{\circ} \mathrm{C}^{\prime}$, Atmospheric Environment, Vol. 16, pp. 2507-2514

Stelson, A. W., Friedlander, S. K. and Seinfeld, J. H. (1979): “A Note on the Equilibrium Relationship between Ammonia and Nitric Acid and Particulate Ammonium nitrate", Atmospheric Environment, Vol. 13, pp. 369-371

Strom, J., Okada, K., and Heintzenberg, J. (1992): "On the State of Mixing of Particles due to Brownian Coagulation", Journal of Aerosol Science, Vol. 23, pp. 467480

Suck, S. H and Brock, J. R. (1979): "Evolution of Atmospheric Aerosol Particle Size Distributions via Brownian Coagulation: Numerical Simulation", Journal of Aerosol Science, Vol. 10, pp. 581-590

Sykes, R. I., Lewellen, W. S., and Parker, S. F., (1984): "A Turbulent Transport Model for Concentration Fluctuations and Fluxes," J. Fluid Mechanics, Vol. 139, pp. 193-218

Tambour, Y., and Seinfeld, J. H. (1980): "Solution of the Discrete Coagulation Equation", Journal of Colloid Interface Science, Vol. 74, pp. 260-272

The Internal Term of General Dynamic Equation of Aerosols, Report to the U.S. EPA, Department of Mechanical Engineering, University of Minnesota, Minneapolis, MN

Tsang, T. H. and Brock, J. R. (1983): "Simulation of Condensation Aerosol Growth by Condensation and Evaporation", Aerosol Science and Technology, Vol. 2, pp. $311-320$

Tsang, T. H. and Brock, J. R. (1984): “On Ostwald Ripening”, Aerosol Science and Technology, Vol. 3, pp. 283-292

Tsang, T. H. and Hippe, J. M. (1988): "Asymptotic Behavior of Aerosol Growth in the Free Molecule Regime", Aerosol Science and Technology, Vol. 8, pp. 265-278

Tsang, T. H. and Rao, A. (1988): "Comparison of Different Numerical Schemes for Condensational Growth of Aerosols", Aerosol Science and Technology, Vol. 9. pp. $271-277$

Tsang, T. H., and Brock, J. R. (1979): "Aerosol Coagulation in the Plume from a Cross-Wind Line Source", Atmospheric Environment, Vol. 16, pp. 2229-2235

Turner, D. B. (1970) "Workbook of Atmospheric Dispersion Estimates Office of Air Program," Publication No. AP-26, U. S. Environmental Protection Agency, Research Triangle Park, NC 27711

Twomey, S. (1964): "Statistical Effects in the Evolution of a Distribution of Cloud Droplets by Coalescence", Journal of Atmospheric Science, Vol. 21, pp. 553-7

Viisanen, Y., Kulmala, M., and Laaksonene, A (1997): "Experiments on GasLiquid Nucleation of Sulfuric Acid and Water", Journal of Chemical Physics, Vol. 107 (3), pp. 920-926

Vuk, C. T., Jones, M. A., and Johnson, J. H. (1976): "The Measurement and Analysis of the Physical Character of Diesel Particulate Emissions", SAE Trans., SAE paper No. 760131, pp. 556-597 
Wang, S. C. and Flagan, R. C. (1990): "Scanning Electrical Mobility Spectrometer", Aerosol Science and Technology, Vol. 13, pp. 230-240

Warheit, D. B., Seidel, W. C., Carakostas, M. C. and Hartsky, M. A. (1990): Attenuation of Perfluoropolymer Fume Pulmonary Toxicity: Effect of Filters, Combustion Method, and Aerosol Age, Pulmonary Toxicity of Perfluoropolymer Fumes, Academic Press, New York, pp. 309-329

Warren, D. R. and Seinfeld, J. H. (1985): "Simulation of Aerosol Size Distribution Evolution in Systems with Simultaneous Nucleation, Condensation, and Coagulation", Aerosol Science and Technology, Vol. 4, pp. 31-43

Weber R. J., Stolzenburg, M. R., Pandis, S. N., and McMurry, P. H. (1998): "Inversion of Ultrafine Condensation Nucleus Counter Pulse Height Distributions to Obtain Nanoparticle ( 3-10 nm) Size Distributions", Journal of Aerosol Science, Vol. 29, pp. 601-615

Weber, R. J., Marti, J. J., McMurry, P. H., Eisele, F. L. Tanner, D. J. and Jefferson, A. (1996): "Measured Atmospheric New Particle Formation Rates: Implications for Nucleation Mechanisms", Chemical Engineering Communications, Vol. 151, pp. 53-64

Weil, J. C., Corio, L. A., Brower, R. P. (1997): "PDF Dispersion Model For Buoyant Plumes In The Convective Boundary Layer", Journal of Applied Meteorology, Vol. 36, No. 8, pp 982-1003

Wexler, A. S. and Seinfeld, J. H. (1990): "The Distribution of Ammonium Salts Among a Size and Composition Dispersed Aerosol", Atmospheric Environment, Vol. 24A, pp. 1231-1246

Wexler, A. S. and Seinfeld, J. H. (1991): "Second-Generation Inorganic Aerosol Model", Atmospheric Environment, Vol. 25A, pp. 2731-2748

Wexler, A. S. and Seinfeld, J. H. (1992): "Analysis of Aerosol Ammonium Nitrate: Departures from Equilibrium during SCAQs", Atmospheric Environment, Vol. 26A, pp. 579-591

Whitby, K. T. (1981): "Determination of Aerosol Growth Rates in the Atmosphere Using Lumped Aerosol Dynamics", Journal of Aerosol Science, Vol. 12, pp. 174-178

Whitby, K. T. (1985): The modal Aerosol Dynamics Model, Part 1. Solution of

Wilemski, G. (1984): "Composition of the Critical Nucleus in Multicomponent Vapor Nucleation", Journal of Chemical physics, Vol. 80, no. 3, pp. 1370-72

Willeke, K., and Baron, P. A. (1992): Aerosol Measurement: Principles Techniques and Applications, Van Nostrand Reinhold, 115 fifth avenue, New York, NY 10003

Yee, E. and Chan, R. (1997): "Simple Model For The Probability Density Function Of Concentration Fluctuations in Atmospheric Plumes", Atmospheric Environment, Vol. 31, No. 7, pp 991-1002

Yom, K., and Brock, J. R. (1984): Private Communication, Department of Chemical Engineering, University of Texas, Austin, TX

Yue, G. and Hamill, P. (1979): "The Homogeneous Nucleation Rates of $\mathrm{H}_{2} \mathrm{SO}_{4}$ $\mathrm{H}_{2} \mathrm{O}$ Aerosol Particles in Air", Journal of Aerosol Science, Vol. 10, pp. 609-614

Zannetti, P. (1990): Air Pollution Modeling - Theories, Computational Methods and Available Software, Van Nostrand Reinhold, NY 
Zebel, G. (1958): "Zur Theories des Koagulation Elektrisch Ungeladener Aerosole", Kolloid Z. , Vol. 156, pp. 102-7

Zebel, G. (1966): Aerosols Science. Edited by C.N.Davies, London, Acedemic Press. 


\section{APPENDIX 1. Fuel Sulfur to $\mathrm{SO}_{4}$ Conversion Equations}

This appendix provides the equations necessary for converting the fuel sulfur to $\mathrm{SO}_{4}$, then to the dilution tunnel $\mathrm{SO}_{4}$ concentration and finally to dilution tunnel $\mathrm{H}_{2} \mathrm{SO}_{4}$ vapor pressure.

Exhaust $\mathrm{SO}_{4}=\left((\right.$ Fuel Rate $) *(\%$ Fuel Sulfur $) *\left(\% \mathrm{~S}\right.$ to $\mathrm{SO}_{4}$ Conversion $) *$

$$
\left.\left(\mathrm{MsO}_{4} / \mathrm{M}_{\text {exhaust }}\right) * 1000000\right) /(\text { Exhaust Rate })
$$

Where the units are:

$$
\begin{aligned}
& \text { Exhaust } \mathrm{SO}_{4}=\mathrm{mg} / \mathrm{m}^{3} \\
& \text { Fuel Rate }=\mathrm{kg} / \mathrm{s} \\
& \text { Fuel Sulfur }=\mathrm{wt} \% \text { sulfur } / 100 \\
& \% \mathrm{~S} \text { to } \mathrm{SO}_{4}=0.04 \text { for this research } \\
& \mathrm{MsO}_{4}=\text { Molecular Weight of } \mathrm{SO}_{4} \\
& \mathrm{M}_{\text {exhaust }}=\text { Molecular Weight of the Exhaust Gas } \\
& \text { Unit Conversion }=1000000 \mathrm{mg} / \mathrm{kg} \\
& \text { Exhaust Rate }=\mathrm{m}^{3} / \mathrm{s}
\end{aligned}
$$

Re-write the equation in terms of fuel/air ratio (F/A).

$$
\mathrm{F} / \mathrm{A}=(\mathrm{kg} / \mathrm{s} \text { fuel }) /(\mathrm{kg} / \mathrm{s} \text { air })
$$

The exhaust rate can be converted to intake air rate by multiplying by:

$$
\text { Intake Air Rate }=\text { Exhaust Rate } *(1-\mathrm{F} / \mathrm{A}) * \text { Density }
$$


Where the unit for the intake air rate is $\mathrm{kg} / \mathrm{s}$, and density of air at standard conditions is $1.186 \mathrm{~kg} / \mathrm{m}^{3}$.

Multiplying Equation 1's numerator and denominator by:

$(1.186)^{*}(1-\mathrm{F} / \mathrm{A})$ and using the $\mathrm{F} / \mathrm{A}$ definition, Equation 1 can be rewritten as:

$$
\begin{aligned}
\text { Exhaust } \mathrm{SO}_{4}= & \left((\mathrm{F} / \mathrm{A}) *(\% \mathrm{Fuel} \text { Sulfur }) *\left(\% \mathrm{~S} \text { to } \mathrm{SO}_{4} \text { conversion }\right) *\right. \\
& \left.\left.\left(\mathrm{MsO}_{4} / \mathrm{M}_{\text {exhaust }}\right) * 1,000,000\right) *(1-\mathrm{F} / \mathrm{A}) * \text { Density }\right)
\end{aligned}
$$

Appropriate dilution ratios is applied to this $\mathrm{SO}_{4}$ concentration.

The last step is to convert the actual diluion tunnel $\mathrm{SO}_{4}$ concentration to $\mathrm{H}_{2} \mathrm{SO}_{4}$ vapor pressure. Using the ideal gas eqution:

$$
\mathrm{P}=(\mathrm{m} / \mathrm{V}) * \mathrm{R} * \mathrm{~T} /(\mathrm{M} * 1000)
$$

Noting that $\mathrm{m} / \mathrm{V}$ is the $\mathrm{H}_{2} \mathrm{SO}_{4}$ concentration, the vapor pressure can be determined:

$$
\begin{aligned}
& \mathrm{P}=\text { pascal }, \mathrm{N} / \mathrm{m}^{2} \\
& \mathrm{~V}=\text { volume }, \mathrm{m}^{3} \\
& \mathrm{R}=8.315 \mathrm{k} \mathrm{N}-\mathrm{m} / \mathrm{kg} \text { mole } \mathrm{K} \\
& \mathrm{M}=\text { molecular weight of exhaust, } \mathrm{kg} / \mathrm{kg} \text { mole } \\
& \mathrm{T}=\text { dilution tunnel temperature }
\end{aligned}
$$




\section{APPENDIX 2. Computer Program}

\section{LIST OF THE MAIN VARIABLES IN THE FORTRAN PROGRAM}

\begin{tabular}{|c|c|}
\hline $\operatorname{beta}(400,400)$ & Beta Kernel \\
\hline betas: & Beta kernel for single/mono disperse particles \\
\hline $\mathrm{Bh}(10):$ & Constants used in the computation of hydration factor \\
\hline $\mathrm{C}:$ & Concentration at any time $(\mathrm{t})$ \\
\hline C0: & Initial concentration \\
\hline dndlogp(400): & $\mathrm{dN} / \operatorname{dlog}\left(\mathrm{d}_{\mathrm{p}}\right)$ \\
\hline dp: & Change in pressure \\
\hline dt: & Time step \\
\hline eta: & Dynamic viscosity \\
\hline$f(400,400,400):$ & Intermediate partition function \\
\hline I1: & Nucleation Rate \\
\hline $\mathrm{kb}:$ & Boltzman constant \\
\hline $\operatorname{Kh}(10)$ : & Constants used in the computation of hydration factor \\
\hline NB: & Number of bins \\
\hline Nh(10): & Number of Hydrates \\
\hline p1: & Partial vapor pressure of hydrates \\
\hline p2: & Partial vapor pressure of sulfates \\
\hline rad: & Radius of the nucleus \\
\hline time: & Time \\
\hline V_rat: & Volume Ratio of the immediate bins \\
\hline $\operatorname{vol}(400):$ & Volume of the bin \\
\hline
\end{tabular}




\section{LISTING OF THE MAIN PROGRAM TO COMPUTE NUCLEATION AND}

\section{COAGULATION RATES}

c IMPLICIT NONE

Real*8 diff(10000), pp2(10) !'total H2SO4 molecules inside hydrates' Real*8 xt(10), xx(10), xn1(10), xn2(10), xc(10), Xi(10),

\& $\mathrm{xdGkT}(10), \mathrm{xr}(10), \mathrm{x}$ ! 'for store results at each step'

Real*8 Kh(10), Nh(10), Bh(10),i1,n1,n2,mw,mn,kk,ka,Nhsum,Nwv,Nav, \& ma,k,na,N2free,dp_change

double precision beta(400,400), dp(400), C0(400), C(400),

$\& d t, \operatorname{vol}(400), p i, \operatorname{rad}(400), f(400,400,400), \mathrm{V}(400,400)$,

\& V_RAT, kb, T, eta, sum_i, sum_j1,sum_j2, time, CS0(3000),

\& CS(3000), betas, dndlogp(400), hours, $\operatorname{radm}(400)$, temp, Tmax,

\& rads(3001), dndlogps(3000), vols(3001), dts, beta_CS0_dt,

\& sigmag,c0_cons, CMD,c0_nucl(400),dia(400),tdia,kn(400),

\& LL(400), cooe(400),gg(400),gtem(400), diffu(400), dtemp1(400),

\& dtemp2(400),dtemp3(400),dtemp10(400),dtemp20(400),dtemp30(400),

\& tdiffu,temp1,temp2,temp3, ac,temp_c1,temp_c

integer $\mathrm{i}, \mathrm{k} 1, \mathrm{j}, \mathrm{NB}, \mathrm{tm}$, temp_i

open (unit=21, file='dk_log.dat', status='unknown')

$\operatorname{open}(9$, file='rh.dat', status='unknown')

C $* * * * * * * * * * *$ INITIALIZATIONS

time $=0.0$ 


$$
\begin{aligned}
& \mathrm{kb}=1.3805 \mathrm{~d}-16 \quad ! 1.3805 \mathrm{~d}-16 \quad \text { ergs } / \mathrm{K} \\
& \mathrm{T}=298.15 \\
& \text { eta }=1.79 \mathrm{~d}-4 \quad \text { ! dynes. } \mathrm{sec} / \mathrm{cm}^{\wedge} 2 \\
& \mathrm{dt}=0.01 \\
& \operatorname{Tmax}=0.01 * 60 / \mathrm{dt} \\
& \text { c } \quad \operatorname{Tmax}=3 / \mathrm{dt} \\
& \mathrm{NB}=120 \quad ! \mathrm{NB}=87 \\
& \operatorname{rad}(1)=5.0 \mathrm{~d}-8 ! \operatorname{rad}(1)=1.0 \mathrm{~d}-8 \\
& \mathrm{CMD}=20.0 \mathrm{e}-7 \\
& \mathrm{pi}=3.1414 \\
& \operatorname{vol}(1)=4 .{ }^{*} \mathrm{pi}{ }^{*} \operatorname{rad}(1) * \operatorname{rad}(1) * \operatorname{rad}(1) / 3.0 \\
& \text { sigmag }=1.8 \\
& \text { c0_cons }=1.0 \mathrm{e}+7 \\
& \text { V_RAT }=1.2 \\
& \text { c } \quad \mathrm{dt}=0.4 \\
& \text { c } \quad \mathrm{T}=319 \\
& \mathrm{pi}=3.14159 \\
& \mathrm{k}=\mathrm{kb} \\
& \mathrm{Na}=6.023 \mathrm{E}+23 \\
& \mathrm{rh}=0.4 \quad ! .4614 \\
& \mathrm{ac}=1.0 \\
& \mathrm{p} 100=23.756
\end{aligned}
$$




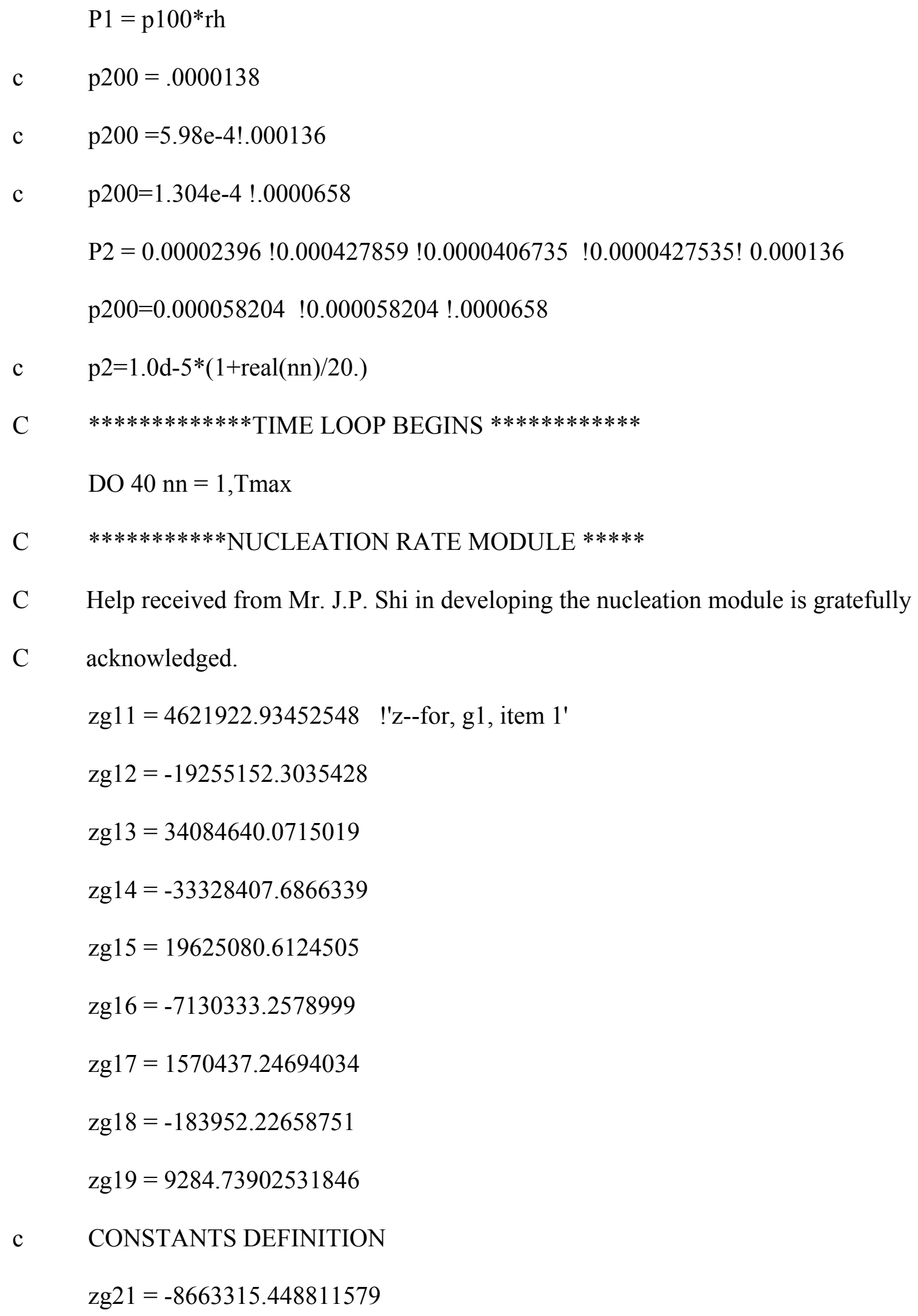




$$
\begin{aligned}
& \operatorname{zg} 22=35426785.3883708 \\
& \operatorname{zg} 23=-61613836.8084201 \\
& \operatorname{zg} 24=59362854.6857313 \\
& \operatorname{zg} 25=-34565413.652318 \\
& \operatorname{zg} 26=12404421.2281494 \\
& \operatorname{zg} 27=-2630033.2324858 \\
& \operatorname{zg} 28=267883.843826984 \\
& \operatorname{zg} 29=-274.627415677067
\end{aligned}
$$

c

$$
\begin{aligned}
\mathrm{zt} 1 & =2.72087172981137 \mathrm{D}-04 \\
\mathrm{zt} 2 & =-1.04544396438644 \mathrm{D}-03 \\
\mathrm{zt} 3 & =1.62574857499663 \mathrm{D}-03 \\
\mathrm{zt} 4 & =-1.30910836608072 \mathrm{D}-03 \\
\mathrm{zt} 5 & =5.76897961472826 \mathrm{D}-04 \\
\mathrm{zt} 6 & =-1.3162673756636 \mathrm{D}-04 \\
\mathrm{zt} 7 & =1.02261406923682 \mathrm{D}-05 \\
\mathrm{zt} 8 & =7.55596366918204 \mathrm{D}-07 \\
\mathrm{zt} 9 & =1.71562456417419 \mathrm{D}-06
\end{aligned}
$$

c

$$
\begin{aligned}
& \mathrm{zv} 1=108.799726968818 \\
& \mathrm{zv} 2=-340.4242486288347 \\
& \mathrm{zv} 3=400.5937205270031 \\
& \mathrm{zv} 4=-220.2310691720908
\end{aligned}
$$




$$
\begin{aligned}
& \mathrm{zv} 5=69.08017850110033 \\
& \mathrm{zv} 6=17.84739439429426 \\
& \mathrm{zv} 7=18.03553337363258
\end{aligned}
$$

C

$$
\begin{aligned}
& \text { zp1 }=-2412.97305638635 \\
& z p 2=3292.71815579841 \\
& z p 3=0 \\
& \text { zp4 }=-2274.36616152316 \\
& \text { zp5 }=1579.10464543587 \\
& \text { zp6 }=-448.965669326196 \\
& \text { zp7 }=48.826568809271
\end{aligned}
$$

c

$$
\begin{aligned}
& \text { restg } 1=8.31 * \mathrm{~T} * \log (\mathrm{rh}) / 4.1868 \\
& \text { restg } 2=8.31 * \mathrm{~T} * \log (\mathrm{P} 2 / \mathrm{p} 200) / 4.1868
\end{aligned}
$$

C

$\mathrm{Kh}(1)=1430.0 / 760$. 'unit:1/mmHg'

$\mathrm{Kh}(2)=54.72 / 760$.

$\operatorname{Kh}(3)=14.52 / 760$

$\operatorname{Kh}(4)=8.12 / 760$

$\operatorname{Kh}(5)=5.98 / 760$.

$\operatorname{Kh}(6)=5.04 / 760$

$\operatorname{Kh}(7)=4.62 / 760$

$\operatorname{Kh}(8)=4.41 / 760$ 


$$
\begin{aligned}
& \mathrm{Kh}(9)=4.28 / 760 . \\
& \mathrm{Kh}(10)=4.27 / 760 .
\end{aligned}
$$

10 DO $100 \mathrm{I}=1,1000000$

$$
\mathrm{x}=.1+\operatorname{real}(\mathrm{i}) / 1000.0
$$

fng1_x $=\operatorname{zg} 11 * \mathrm{x} * * 8+\mathrm{zg} 12 * \mathrm{x} * * 7+\mathrm{zg} 13 *$

$\& \mathrm{x} * * 6+\mathrm{zg} 14 * \mathrm{x} * * 5+\mathrm{zg} 15 * \mathrm{x} * * 4+\mathrm{zg} 16 * \mathrm{x} * * 3+\mathrm{zg} 17$

$\& * \mathrm{x} * * 2+\mathrm{zg} 18 * \mathrm{x}+\mathrm{zg} 19$

$$
\text { fng2_x }=\mathrm{zg} 21 * \mathrm{x} * * 8+\mathrm{zg} 22 * \mathrm{x} * * 7+\mathrm{zg} 23 * \mathrm{x} * * 6+
$$

$\& \mathrm{zg} 24 * \mathrm{x} * * 5+\mathrm{zg} 25 * \mathrm{x} * * 4+\mathrm{zg} 26 * \mathrm{x} * * 3+$

$\& \operatorname{zg} 27 * x * * 2+\operatorname{zg} 28 * x+z g 29$

fnt_x $=\mathrm{zt} 1 * \mathrm{x} * * 8+\mathrm{zt} 2 * \mathrm{x} * * 7+\mathrm{zt} 3 * \mathrm{x} * * 6+\mathrm{zt} 4 * \mathrm{x} * * 5+$ $\& \mathrm{zt} 5 * \mathrm{x} * * 4+\mathrm{zt} 6 * \mathrm{x} * * 3+\mathrm{zt} 7 * \mathrm{x} * * 2+\mathrm{zt} 8 * \mathrm{x}+\mathrm{zt} 9$

fndt_x $=8 * \mathrm{zt} 1 * \mathrm{x} * * 7+7 * \mathrm{zt} 2 * \mathrm{x} * * 6+6 * \mathrm{zt} 3 * \mathrm{x} * * 5+$ \& $5 * \mathrm{zt} 4 * \mathrm{x} * * 4$ $\&+4 * \mathrm{zt} 5 * \mathrm{x} * * 3+3 * \mathrm{zt} 6 * \mathrm{x} * * 2+2 * \mathrm{zt} 7 * \mathrm{x}+\mathrm{zt} 8$

$\mathrm{fnv} \_\mathrm{x}=\mathrm{zv} 1 * \mathrm{x} * * 6+\mathrm{zv} 2 * \mathrm{x} * * 5+\mathrm{zv} 3 * \mathrm{x} * * 4+\mathrm{zv} 4 * \mathrm{x} * * 3+$ $\& \mathrm{zv} 5 * \mathrm{x} * * 2+\mathrm{zv} 6 * \mathrm{x}+\mathrm{zv} 7$ 
$\mathrm{fndv} \_\mathrm{x}=6 * \mathrm{zv} 1 * \mathrm{x} *{ }^{*} 5+5 * \mathrm{zv} 2 * \mathrm{x} * 4+4 * \mathrm{zv} 3 * \mathrm{x} * * 3+$ $\& \quad 3 * \mathrm{zv} 4 * \mathrm{x} * * 2+2 * \mathrm{zv} 5 * \mathrm{x}+\mathrm{zv} 6$

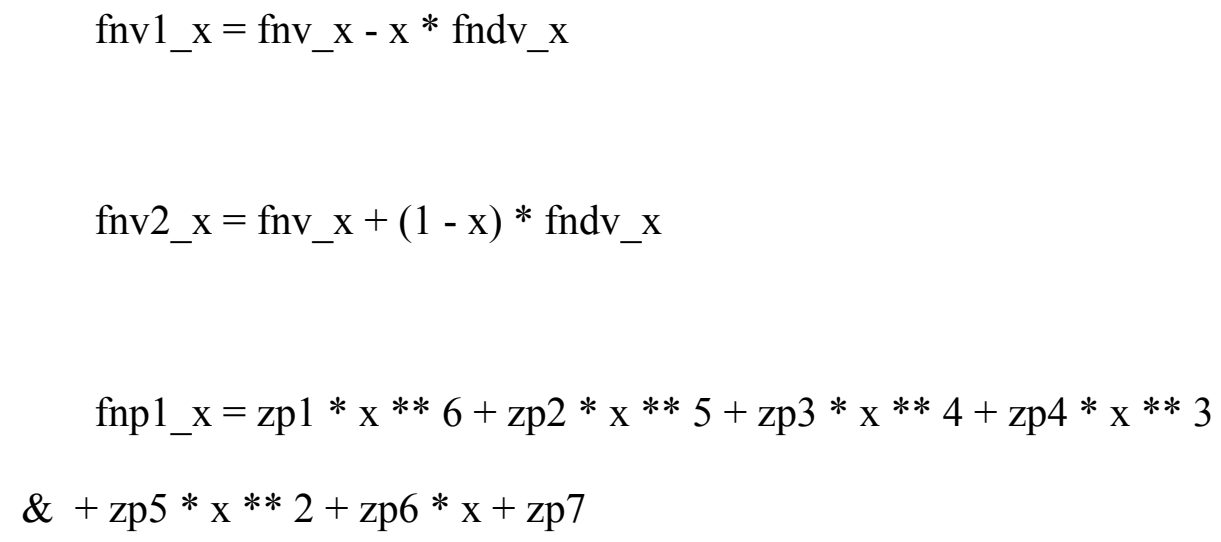


110 continue

c WRITE $(*, *)$ "c1=",c1 ,"c2=", c2,ps,fng1_x,restg1

$$
\mathrm{Ch}=((1+\mathrm{c} 1) /(1+\mathrm{c} 2))
$$

$\mathrm{P} 2$ free $=\mathrm{P} 2 /(1+\mathrm{c} 2)$

$\mathrm{N} 2$ free $=\mathrm{Na} * \mathrm{P} 2$ free ${ }^{*} 1.0 /(62400.0 * \mathrm{~T})$

c $\quad$ WRITE $(*, *)$ " Ch=",(Ch), "P2free=", P2free

$\operatorname{diff}(\mathrm{i})=\mathrm{fnv} 2 \_\mathrm{x} * \operatorname{diatG} 1-\mathrm{fnv} 1 \_\mathrm{x} *$

$\&(\operatorname{diatG} 2-8.314 * \mathrm{~T} * \mathrm{LOG}(\mathrm{Ch}) / 4.1868)$

IF ((diff(i)*diff(i-1) .lt. 0)) THEN

GO TO 20

END IF

c $\quad$ WRITE $(*, *)$ "step",i ," x = ", x, diff(i)

100 continue

20 continue

c

$\mathrm{r}=-2 . *$ fnt_x*fnv_x $/((1-\mathrm{x}) * \operatorname{diatG} 1+\mathrm{x} *(\operatorname{diatG} 2-8.314 * \mathrm{~T} * \log (\mathrm{Ch}) / 4.1868))$

$\mathrm{n} 1=4 . / 3 \cdot{ }^{*} \mathrm{pi}^{*} \mathrm{r}^{* *} 3 . * \mathrm{Na} /\left(\mathrm{fnv} 1 \_\mathrm{x}+\mathrm{x} * \mathrm{fnv} 2 \_\mathrm{x} /(1 .-\mathrm{x})\right)$

$\mathrm{n} 2=\mathrm{x} /(1-\mathrm{x})^{*} \mathrm{n} 1$

WRITE $(*, *)$ "n1=",n1 ,"n2=", n2, "r=", r

$\mathrm{PP}=1$

Nhsum $=0$

DO $120 \mathrm{i}=1,10$

$\mathrm{PP}=\mathrm{PP} * \mathrm{Kh}(\mathrm{i}) * \mathrm{P} 1$ 
\& "Nhsum=", Nhsum

$$
\operatorname{diatG}=(\mathrm{n} 1 * \operatorname{diatG} 1+\mathrm{n} 2 * \operatorname{diatG} 2) / \mathrm{Na}+4 \cdot{ }^{*} \mathrm{pi}{ }^{*} \mathrm{r}^{*} 2 . * \text { fnt } \_\mathrm{x}
$$

WRITE $(*, *)$ "diat\#G=",(4.0)/3.*pi*r**2.*fnt_x,diatG

C

$$
\begin{aligned}
& * * * * * * * * * N U C L E A T I O N \text { RATE }(\mathrm{I} 1) * * * * * * * * * * * * * * * * * \\
& \text { i1 = C_NUC*EXP(-diatG*4.1868E+07/(k*T)) } \\
& \text { WRITE }(*, *) \text { "n1=",(n1),"n2=", n2, "r=", r } \\
& \text { WRITE }(*, *) ~ " \mathrm{n} 1 * \operatorname{diatG} 1=",(\mathrm{n} 1) * \operatorname{diatG} 1, " \mathrm{n} 2 * \operatorname{diatG} 2=", \mathrm{n} 2 * \operatorname{diatG} 2,
\end{aligned}
$$

$\&$ "4pir**2t=", 4. * pi * r **2.*fnt_x

WRITE $(*, *)$ "diatG/kt=",(diatG) *4.1868E+07/k/T,"I1= ", i1,

$\&$ "(cm-3.s-1)"

WRITE $(*, *)$ "fng1=",fng1_x ,"fng2=", fng2_x

WRITE $(*, *)$ "fnv=",(fnv_x $), " f n t="$, fnt_x, "fnv1_x=",

\& fnv1_x, "fnv2_x=", fnv2_x

WRITE $(*, *)$ "n1v1+n2v2=",((n1*fnv1_x+n2*fnv2_x ))/Na,"4/3*pi* r**3=",

\& 4. /3.* pi $* \mathrm{r} * * 3$.

WRITE $(*, *)$ "(2386n1v1+1500n2v2)/(4/3*pi)**.33333=",

$\&\left(\left(\left(2386 . *_{n} 1 *\right.\right.\right.$ fnv1_x $+1500 . *_{n} 2 *$ fnv2_x $\left.\left.) / \mathrm{Na} /(4 . / 3 . * \mathrm{pi})\right)\right) * * .33333$

c $\quad$ write $(9,5) \mathrm{p} 2, \mathrm{i} 1,\left(2 *^{*} \mathrm{r}\right)$

5 format(e13.6,2x,e13.6,2x, e13.6, 2x, e13.6,2f8.4)

$\mathrm{C} * * * * * * * * * * * * * \mathrm{COAGULATION}$ MODULE $* * * * * * * * * * * * * * * * * * * * * * * * * * * *$

139 continue

C $* * * * * * * * * * *$ INITIAL BIN DEFINITION 
if (time .eq. 0.0) then

DO $199 \mathrm{i}=2,3000$

if (i .lt. $(\mathrm{NB}+2))$ then

$\mathrm{C} 0(\mathrm{i})=0.0$

$\operatorname{rad}(\mathrm{i})=\operatorname{rad}(1) *\left(\mathrm{~V} \_\mathrm{RAT} * *((\mathrm{i}-1) / 3.0)\right)$

$\operatorname{vol}(\mathrm{i})=\operatorname{vol}(1) * \mathrm{~V}_{-} \mathrm{RAT} * *(\mathrm{i}-1)$

c $\quad \mathrm{C} 0(\mathrm{i})=((\log (2 * \operatorname{rad}(\mathrm{i}))-\log (\mathrm{CMD})) * * 2) /(2 * \log ($ sigmag $) * \log ($ sigmag $))$

c $\quad \mathrm{c} 0(\mathrm{i})=\left(\mathrm{c} 0 \_\right.$cons $\left.*(\exp (-\mathrm{c} 0(\mathrm{i})))\right) /\left((2 * \mathrm{pi})^{* *} 0.5\right)$

c $\quad \mathrm{c} 0(\mathrm{i})=\mathrm{c} 0(\mathrm{i}) / \log ($ sigmag $)$

c $\quad \operatorname{write}\left(21,{ }^{*}\right)(2 * \operatorname{rad}(\mathrm{i})), \mathrm{c} 0(\mathrm{i})$

c $\quad \mathrm{c} 0(\mathrm{i})=\mathrm{c} 0(\mathrm{i}) *(\log (\operatorname{rad}(\mathrm{i}) / \operatorname{rad}(\mathrm{i}-1)))$

temp $=\log (10.0)$

end if

$\operatorname{vols}(\mathrm{i})=i * \operatorname{vol}(1)$

$\operatorname{rads}(\mathrm{i})=\left(\left(0.75^{*} \operatorname{vols}(\mathrm{i}) / \mathrm{pi}\right)^{* *}(1 . / 3).\right)$

199 CONTINUE

c end if

c $\quad \mathrm{c} 0(1)=1.0 \mathrm{~d}+6$

c $\quad \mathrm{c} 0(1)=1.0 \mathrm{~d}+6$

$\mathrm{C}(1)=\mathrm{c} 0(1)$ 
$\operatorname{cs} 0(1)=\operatorname{co}(1)$

$\operatorname{cs}(1)=\operatorname{co}(1)$

do $300 \mathrm{j}=1, \mathrm{NB}$

do $300 \mathrm{i}=1, \mathrm{NB}$

$V(i, j)=\operatorname{vol}(i)+\operatorname{vol}(j)$

300 continue

do $2000 \mathrm{k} 1=1, \mathrm{NB}$

do $3000 \mathrm{j}=1, \mathrm{NB}$

do $4000 \mathrm{i}=1$,NB

$\mathrm{f}(\mathrm{i}, \mathrm{j}, \mathrm{k} 1)=0.0$

if $(((\mathrm{V}(\mathrm{i}, \mathrm{j}) . \mathrm{LT} . \operatorname{vol}(\mathrm{k} 1+1))$

\& .AND. (V(i,j) .ge. vol(k1)))) THEN

$\mathrm{f}(\mathrm{i}, \mathrm{j}, \mathrm{k} 1)=(\operatorname{vol}(\mathrm{k} 1+1)-\mathrm{V}(\mathrm{i}, \mathrm{j}))^{*} \operatorname{vol}(\mathrm{k} 1) /$

$\&\left((\operatorname{vol}(\mathrm{k} 1+1)-\operatorname{Vol}(\mathrm{k} 1))^{*} \mathrm{~V}(\mathrm{i}, \mathrm{j})\right)$

if $(k 1$.eq. $N B) f(i, j, k 1)=0.0$

end if

if $((\mathrm{V}(\mathrm{i}, \mathrm{j}) \cdot \mathrm{LT} \cdot \mathrm{vol}(\mathrm{k} 1))$

\& .AND. (V(i,j) .GT. vol(k1-1))) THEN

$f(i, j, k 1)=1-f(i, j, k 1-1)$

if $(\mathrm{k} 1$.eq. 1) $\mathrm{f}(\mathrm{i}, \mathrm{j}, \mathrm{k} 1)=0.0$

end if

if $((\mathrm{k} 1$.eq. NB) .AND. $((\mathrm{V}(\mathrm{i}, \mathrm{j})$.ge. vol(NB))) $)$ THEN 


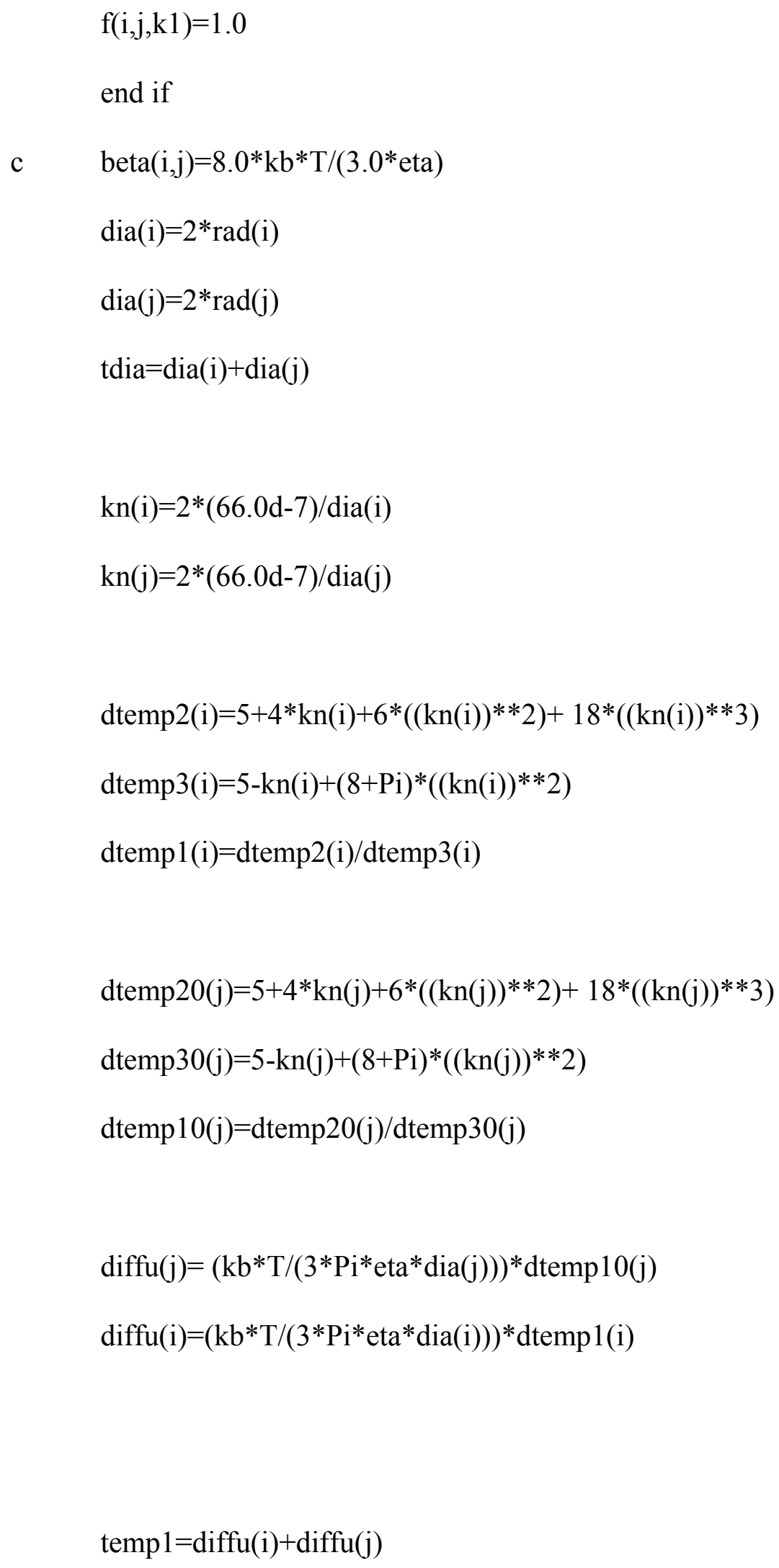




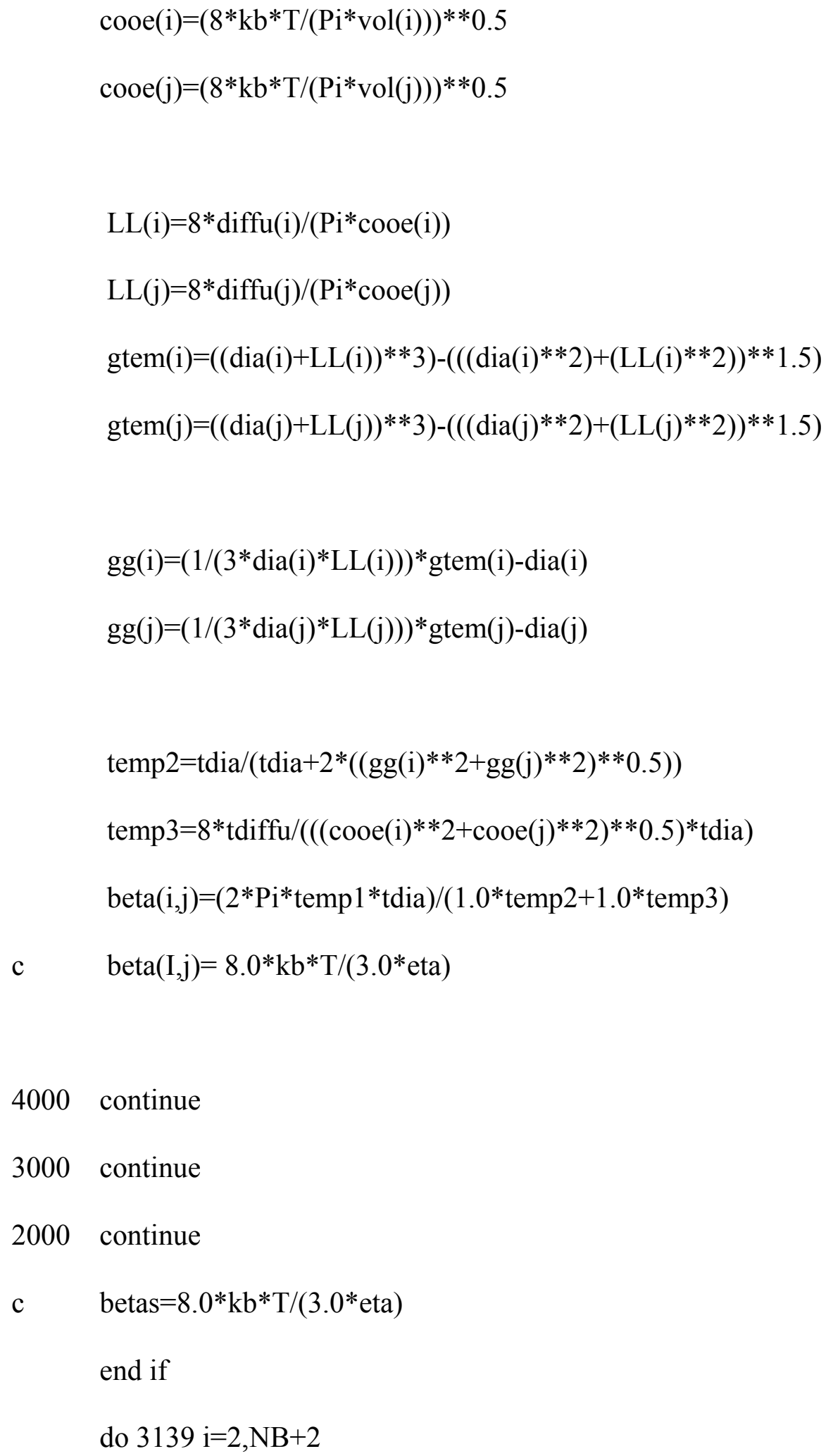




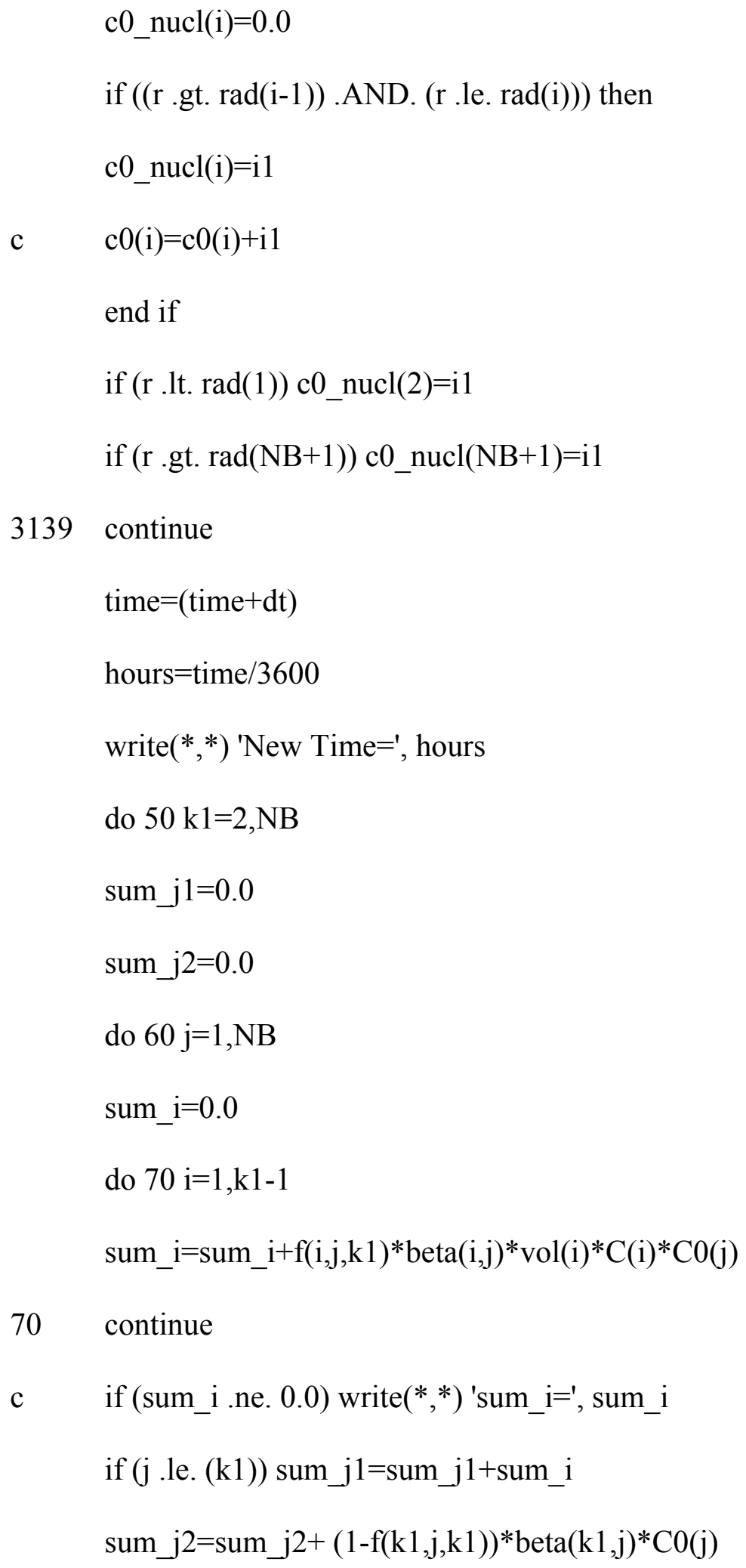


if $(\mathrm{k} 1$.ne. 2) then

temp_c $=\mathrm{ac} * \operatorname{beta}(2,(\mathrm{k} 1-1)) * \mathrm{c} 0(2) * \mathrm{c} 0(\mathrm{k} 1-1) * \mathrm{dt} * \operatorname{vol}(\mathrm{k} 1-1)$

c $\quad \& !+\operatorname{beta}(2, \mathrm{k} 1) * \mathrm{c} 0(2) * \mathrm{c} 0(\mathrm{k} 1) * \mathrm{dt} * \operatorname{vol}(\mathrm{k} 1)))$

temp_c1 $=\operatorname{beta}(2, \mathrm{k} 1) * \mathrm{c}(2) * \mathrm{dt}$

if (temp_c .ne. 1e+4) write(*,*)'temp_c=', (temp_c/dt/vol(k1)), k1

end if

$\mathrm{C}(\mathrm{k} 1)=\left(\operatorname{vol}(\mathrm{k} 1) * \mathrm{c} 0(\mathrm{k} 1)+\mathrm{dt} * \operatorname{sum} \mathrm{j} 1+\mathrm{dt} * \operatorname{vol}(\mathrm{k} 1) * \mathrm{c} 0 \_\right.$nucl $(\mathrm{k} 1)+$

\& temp_c)/

$\&\left(1.0+d t * s u m \_j 2+\right.$ temp_c1 $) / \operatorname{vol}(\mathrm{k} 1)$

50 continue

do $80 \mathrm{k} 1=2, \mathrm{NB}$

c $\quad \operatorname{dndlogp}(\mathrm{k} 1)=\mathrm{c}(\mathrm{k} 1) /(\log (\operatorname{rad}(\mathrm{k} 1+1) / \operatorname{rad}(\mathrm{k} 1)))$

$\operatorname{dndlogp}(\mathrm{k} 1)=\mathrm{c}(\mathrm{k} 1) /(\log (\operatorname{rad}(\mathrm{k} 1) / \operatorname{rad}(\mathrm{k} 1-1)))$

$\operatorname{dndlogps}(\mathrm{k} 1)=\mathrm{cs}(\mathrm{k} 1) / \log (\operatorname{rads}(\mathrm{k} 1+1) / \operatorname{rads}(\mathrm{k} 1))$

$\mathrm{c} 0(\mathrm{k} 1)=\mathrm{c}(\mathrm{k} 1)$

$\operatorname{cs} 0(\mathrm{k} 1)=\operatorname{cs}(\mathrm{k} 1)$

$\operatorname{radm}(\mathrm{k} 1)=\operatorname{rad}(\mathrm{k} 1) * 0.02$

temp $=(\log (\operatorname{rad}(\mathrm{k} 1+1) / \operatorname{rad}(\mathrm{k} 1)))$

if $(\bmod (\mathrm{nn}, 1)$.eq. 0$)$ then

write(21,84)k1,hours,radm(k1), dndlogp(k1),i1,r

end if

write $(*, *) \operatorname{radm}(\mathrm{k} 1), \operatorname{dndlogp}(\mathrm{k} 1)$

84 format $(2 x, 16,2 x, e 13.6$, e13.6, 2x, e13.6,2x, e13.6,2x, e13.6) 
80 continue

90 continue

dp_change $=\mathrm{i} 1 * \mathrm{k} * \mathrm{~T} *(1.0 *(1.0 * \mathrm{n} 2+0.0 * \mathrm{n} 1)) * \mathrm{dt} * 760.0 /(1.01 \mathrm{e}+6)$

p2=p2-dp_change

write $(*, *)$ time, p2,i1,r,dp_change

write $(9,73)(1000 *$ time $), 11,((0.02 \mathrm{e}+9) * \mathrm{r}), \mathrm{p} 2, \mathrm{n} 1, \mathrm{n} 2$

73 format (f7.3,2x,e13.6,2x,e13.6,2x,e13.6,2x,f5.2,2x,f5.2)

40 continue

STOP

END 


\section{PUBLICATIONS RESULTING FROM THIS WORK}

1. "On the Prediction of Concentration Variations in a Dispersing Heavy-Duty Truck Exhaust Plume Using K- $\varepsilon$ Turbulent Closure," Atmospheric Environment, Vol. 35(31), pp. 5267-75 (2001)

2. "Modeling Nucleation and Coagulation Modes in the Formation of Particulate Matter Inside a Turbulent Exhaust Plume of a Diesel Engine," J. Colloid and Interface Science, Vol. 249(1), pp. 96-103 (2002)

3. "Effect of Ambient Dilution on Coagulation of Particulate Matter in a Turbulent Dispersing Plume”, Air Emissions From Mobile Sources - Recent Data and Trends (Session Code: ENV4) at SAE Congress 2002, Cobo Center, Detroit, MI, March 4-7, 2002, SAE Paper Number 2002-01-0652

4. "Prediction of Pollutant Concentration Variation Inside a Turbulent Dispersing Plume using PDF and Gaussian Models", Air Emissions From Mobile Sources Recent Data and Trends (Session Code: ENV4) at SAE Congress 2002, Cobo Center, Detroit, MI, March 4-7, 2002, SAE Paper Number 2002-01-0654

5. "Effect of Soot on $\mathrm{NO}_{\mathrm{x}}$ in Industrial Furnaces," AFRC Fall Symposium, San Francisco, CA, October 4, 1999 